\title{
Guide opérationnel CITE 2011 DIRECTIVES POUR LA CLASSIFICATION DES PROGRAMMES ÉDUCATIFS NATIONAUX ET DES CERTIFICATIONS CORRESPONDANTES
}





\title{
GUIDE OPÉRATIONNEL CITE 2011
}

\author{
DIRECTIVES POUR LA CLASSIFICATION \\ DES PROGRAMMES ÉDUCATIFS NATIONAUX \\ ET DES CERTIFICATIONS CORRESPONDANTES
}

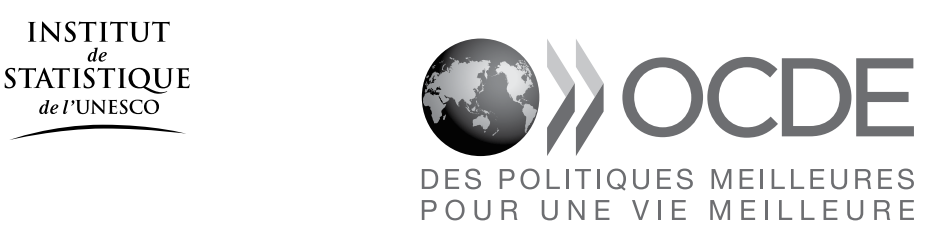


Cet ouvrage est publié sous la responsabilité du Secrétaire général de l'OCDE. Les opinions et les interprétations exprimées ne reflètent pas nécessairement les vues des pays membres de l'OCDE, de I'Union européenne ou de l'Institut de statistique de l'UNESCO.

Ce document et toute carte qu'il peut comprendre sont sans préjudice du statut de tout territoire, de la souveraineté s'exerçant sur ce dernier, du tracé des frontières et limites internationales, et du nom de tout territoire, ville ou région.

ISU ISBN 978-92-9189-180-1

OCDE ISBN 978-92-64-24883-0 (imprimé)

OCDE ISBN 978-92-64-24882-3 (PDF)

Les données statistiques concernant Israël sont fournies par et sous la responsabilité des autorités israéliennes compétentes. L'utilisation de ces données par l'OCDE est sans préjudice du statut des hauteurs du Golan, de Jérusalem Est et des colonies de peuplement israéliennes en Cisjordanie aux termes du droit international.

La version originale en anglais a été publiée par l'OCDE sous le titre ISCED 2011 Operational Manual: Guidelines for Classifying National Education Programmes and Related Qualifications @ OECD, European Union, UNESCOUIS 2015.

(c) UNESCO-ISU, OCDE 2015 pour l'édition en français.

Cet ouvrage étant soumis à la licence Creative Commons Attribution-Non commerciale-pas de travaux dérivés 3.0 IGO (CC BY-NC-ND 3.0 IGO) http://creativecommons.org/licenses/by-nc-nd/3.0/igo/deed.en, vous êtes libre de copier et de diffuser le matériel, pour autant que son utilisation soit à des fins non commerciales, sous réserve des conditions suivantes :

Attribution - Merci de citer cet ouvrage comme suit : Institut de statistique de l'UNESCO et OCDE (2015), Manuel opérationnel CITE 2011 : Directives pour la classification des programmes éducatifs nationaux et des certifications correspondantes, Montréal : Institut de statistique de l'UNESCO http://dx.doi. org/10.15220/978-92-9189-180-1-fre Attribution Creative Commons CC BY-NC-ND 3.0 IGO.

Contenu appartenant à des tiers - Institut de statistique de l'UNESCO et OCDE ne détiennent pas nécessairement chaque composante du contenu du présent ouvrage. Dès lors, ni l'OCDE, ni l'Institut de statistique de l'UNESCO ne peuvent garantir que l'utilisation de toute composante ou partie contenue dans le présent ouvrage et détenue par des tiers ne porte pas atteinte aux droits desdits tiers. Vous devez assumer seul le risque de plainte résultant de cette atteinte. Si vous souhaitez réutiliser une composante du présent ouvrage, il vous incombe de déterminer si une autorisation est nécessaire en vue de ce réemploi et d'obtenir cette autorisation de la part du détenteur du droit d'auteur. Les exemples de composantes sont, entre autres, des tableaux, des chiffres ou des illustrations. Les demandes pour usage commercial ou les questions relatives aux droits et aux licences devront être adressées à uis.publications@unesco.org. Les demandes d'autorisation de photocopier une partie de ce contenu à des fins commerciales peuvent être obtenues auprès de Copyright Clearance. 


\section{Avant-propos}

La structure des systèmes éducatifs varie énormément d'un pays à l'autre. Afin de produire des statistiques et des indicateurs qui soient de nature comparable à l'échelle internationale, il est nécessaire de définir un cadre permettant de collecter et de soumettre des données sur les programmes éducatifs (et les certifications qui en résultent) dont les contenus sont de niveau similaire. C'est dans ce but que la Classification Internationale Type de l'Éducation (CITE) a été créée : il s'agit d'une classification officielle utilisée pour classer et présenter des statistiques relatives à l'éducation comparables à l'échelle internationale.

Elle a d'abord été développée par l'UNESCO dans les années 1970, puis révisée une première fois en 1997. Étant donné l'évolution des systèmes d'éducation et d'enseignement au début du $21^{e}$ siècle, une nouvelle révision de la CITE a été menée à bien entre 2009 et 2011, sur la base de concertations globales avec les pays, des experts régionaux et des organisations internationales. Cette révision prend en compte des évolutions importantes dans la structure de l'enseignement supérieur telles que le processus de Bologne en Europe, le développement des programmes éducatifs pour la petite enfance et un intérêt accru pour les statistiques relatives aux résultats de l'éducation (par exemple le niveau d'éducation atteint). La CITE 2011 révisée a été adoptée par la Conférence générale de l'UNESCO à sa 36e session en novembre 2011.

Le présent Guide opérationnel CITE 2011 : Directives pour la classification des programmes éducatifs nationaux et des certifications correspondantes a été préparé conjointement par l'Institut de statistique de l'UNESCO (ISU), I'OCDE et Eurostat, et intègre de multiples concertations sur les cartographies des systèmes éducatifs nationaux de la CITE 2011. Cet ouvrage a été préparé sous la responsabilité d'Alison Kennedy de l'Institut de statistique de l'UNESCO (ISU), d'Éric Charbonnier et de Nhung Truong de la Division Innovation et Mesure du Progrès (IMEP) de la Direction de l'éducation et des compétences de l'OCDE et de Marta Beck-Domżalska d'Eurostat.

Le présent Guide opérationnel vise à apporter un soutien aux pays dans l'implémentation de la CITE 2011 et à s'assurer que les cartographies des systèmes éducatifs nationaux du cadre révisé de la CITE soient transparentes. L'implémentation de la CITE 2011 devrait être à la fois un processus itératif et interactif, dans le cadre duquel le consensus sur les cartographies peut être développé entre les pays et les organisations internationales, en vue d'améliorer la comparabilité internationale dans les statistiques de l'éducation soumises

La publication du présent Guide opérationnel constitue une étape importante dans un processus consultatif de long terme visant à améliorer en permanence la comparabilité des statistiques internationales sur l'éducation. 


\section{Remerciements}

De nombreuses personnes ont contribué à l'élaboration de cet ouvrage.

Nous remercions tout particulièrement tous les pays pour la production de leurs cartographies nationales de la CITE sans laquelle cet ouvrage n'aurait pas été possible.

L'OCDE souhaite remercier tous les experts et institutions actifs dans le cadre du groupe de travail de l'OCDE Indicateurs des systèmes éducatifs (INES), les représentants du réseau INES sur le marché du travail et les retombées socioéconomiques sur l'éducation (LSO) et les participants au groupe de travail informel INES Éducation de la petite enfance pour leur inestimable contribution.

Eurostat souhaite remercier les membres du groupe de travail Statistiques de l'Éducation et de la Formation (ETS) qui ont exprimé leur avis sur diverses parties du Guide et tous les collègues des pays qui ont collaboré à l'analyse des cartographies de la CITE.

L'Institut de statistique de l'UNESCO (ISU) souhaite remercier tous les experts nationaux ayant participé à des ateliers et soumis des cartographies pour révision. L'ISU souhaite également remercier le Gouvernement japonais pour son soutien financier dans l'implémentation de la CITE 2011 dans la région Asie et Pacifique par le biais de fonds japonais en dépôt à l'UNESCO, ainsi que l'Organisation de la Ligue arabe pour l'éducation, la culture et la science (ALECSO) qui a financé des ateliers de formation régionaux pour les États arabes.

Toute notre gratitude va également aux délégués français pour la préparation du tableau récapitulatif des codes et des critères de la CITE 2011 (Annexe A du Guide opérationnel) et aux nombreux autres collègues ayant apporté une contribution non négligeable ou exprimé un avis ayant contribué à la préparation de ce Guide. 


\section{Table des matières}

PARTIE I APERÇU DE LA CITE 2011

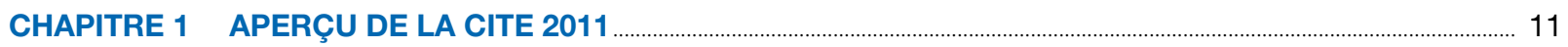

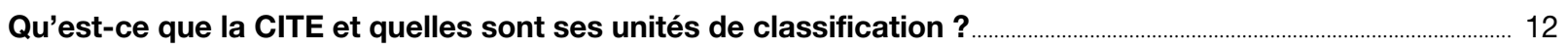

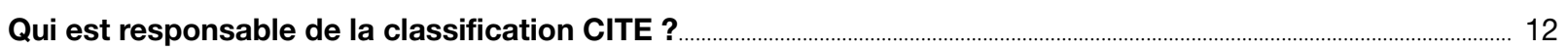

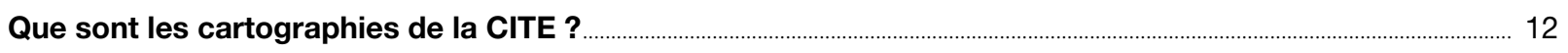

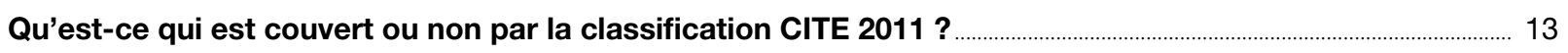

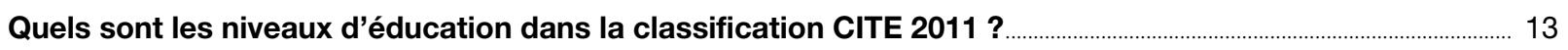

Comment les programmes et les certifications correspondantes sont-ils affectés aux niveaux de la CITE 2011 ?

Comment les programmes qui couvrent plusieurs niveaux de la CITE, qui sont séquentiels ou modulaires, sont-ils classés dans la CITE 2011?

Par quelles dimensions complémentaires les programmes et les certifications peuvent-ils être

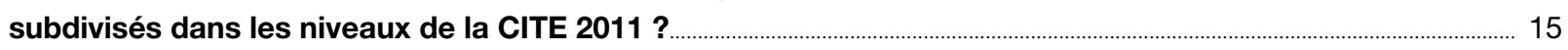

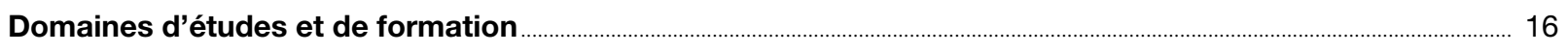

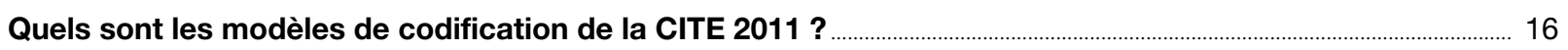

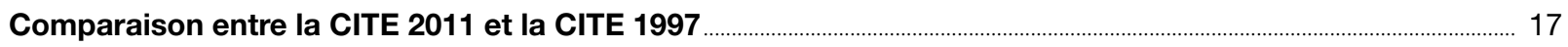

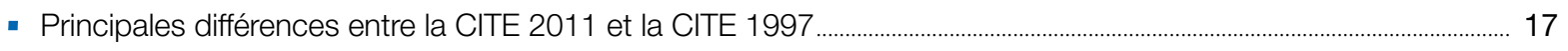

- Tableaux de correspondance entre les révisions de la CITE ...................................................................................... 18

PARTIE II NIVEAUX 0 À 4 DE LA CITE 2011 : DE L’ÉDUCATION DE LA PETITE ENFANCE À L'ENSEIGNEMENT POST-SECONDAIRE NON-SUPÉRIEUR ……................................................................ 21

CHAPITRE 2 NIVEAU 0 DE LA CITE 2011: ÉDUCATION DE LA PETITE ENFANCE ................................................ 21

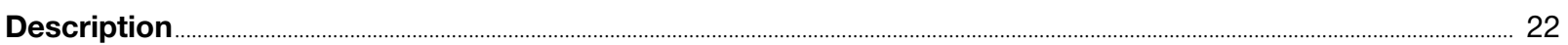

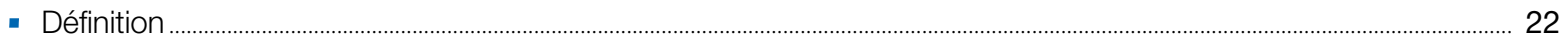

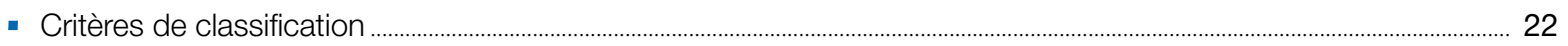

- Dimensions complémentaires ............................................................................................................................................................ 23

Correspondances entre la CITE 2011 et la CITE 1997 ...................................................................................................... 23

Directives pour la classification des programmes et de leurs certifications reconnues correspondantes au niveau 0 de la CITE

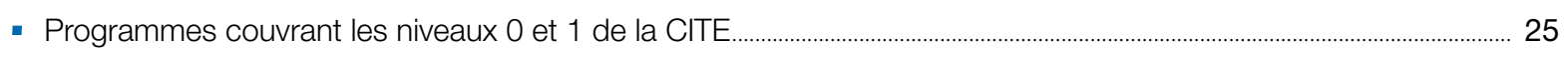

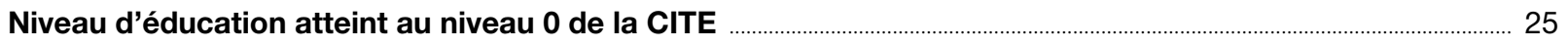

Exemples de programmes et de certifications classés au niveau $\mathbf{0}$ de la CITE ……............................................. 26

CHAPITRE $3 \quad$ NIVEAU 1 DE LA CITE 2011 : ENSEIGNEMENT PRIMAIRE ................................................................ 31

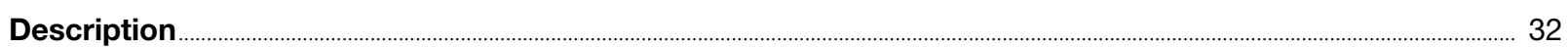

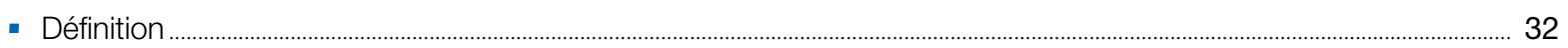

- Critères de classification $(§ 124)$............................................................................................................................................................. 32

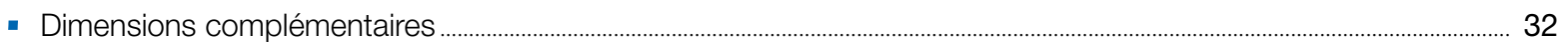


Directives pour la classification des programmes et de leurs certifications reconnues correspondantes au niveau 1 de la CITE.

- Programmes couvrant l'enseignement primaire et un autre niveau de la CITE .............................................................. 34

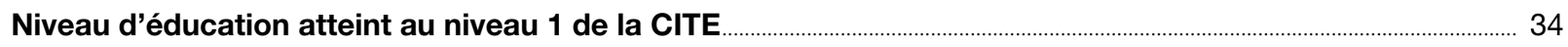

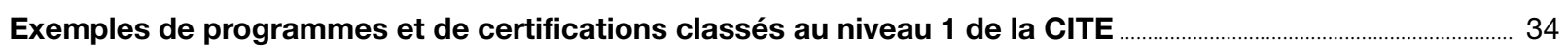

CHAPITRE 4 NIVEAU 2 DE LA CITE 2011 : PREMIER CYCLE DE L'ENSEIGNEMENT SECONDAIRE ........... 41

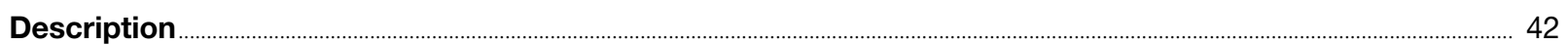

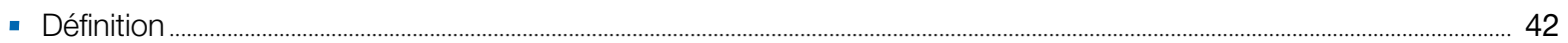

- Critères de classification $(\S 143)$.......................................................................................................................................................... 42

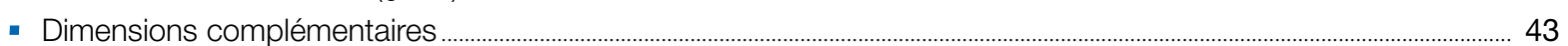

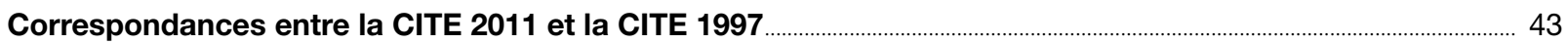

Directives pour la classification des programmes et de leurs certifications au niveau 2 de la CITE ................ 44

- Programmes couvrant le premier cycle de l'enseignement secondaire et un autre niveau de la CITE ..................... 44

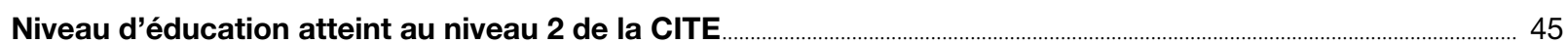

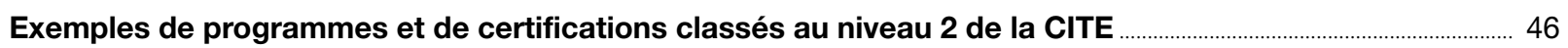

CHAPITRE 5 NIVEAU 3 DE LA CITE 2011 : DEUXIÈME CYCLE DE L'ENSEIGNEMENT SECONDAIRE........ 51

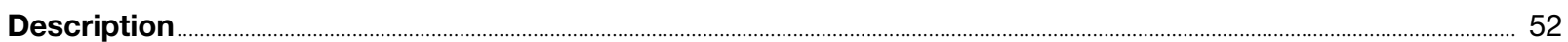

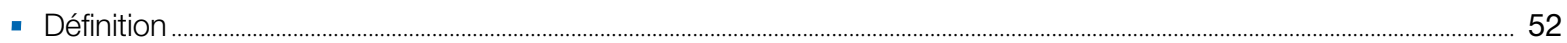

- Critères de classification $(\S 166)$.............................................................................................................................................................. 52

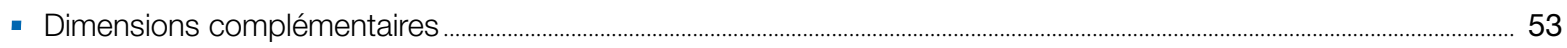

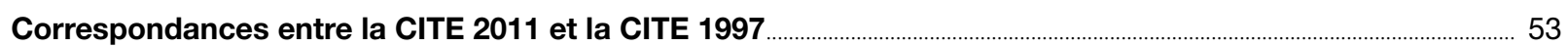

Directives pour la classification des programmes et de leurs certifications au niveau 3 de la CITE ................ 54

- Programmes couvrant le deuxième cycle de l'enseignement secondaire et un autre niveau de la CITE .................. 55

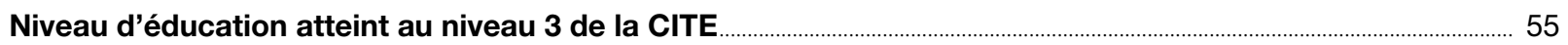

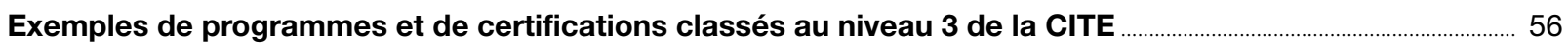

CHAPITRE 6 NIVEAU 4 DE LA CITE 2011 : ENSEIGNEMENT POST-SECONDAIRE NON-SUPÉRIEUR ........ 63

Description

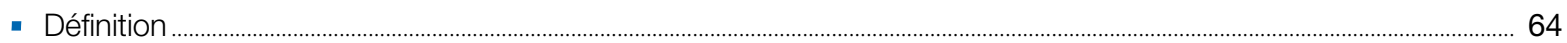

- Critères de classification $(\$ 189)$................................................................................................................................................................ 64

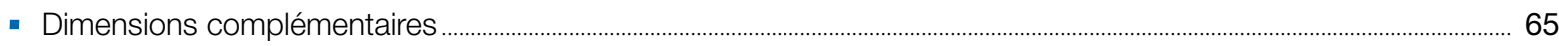

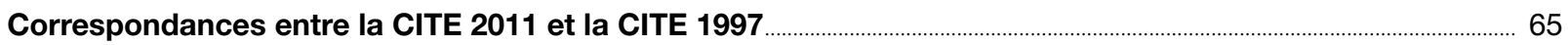

Directives pour la classification des programmes et de leurs certifications reconnues correspondantes au niveau 4 de la CITE

- Différence entre les niveaux 4 et 5 de la CITE .............................................................................................................................. 67

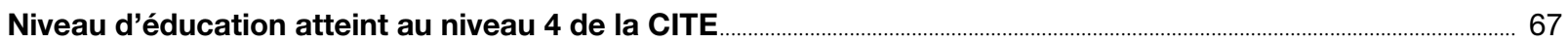

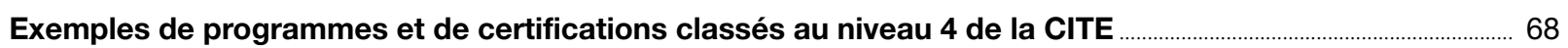

PARTIE III NIVEAUX 5 À 8 DE LA CITE : ENSEIGNEMENT SUPÉRIEUR ...................................................................... 73

CHAPITRE 7 APERÇU DE LA CITE 2011 NIVEAUX DE L'ENSEIGNEMENT SUPÉRIEUR .................................. 73

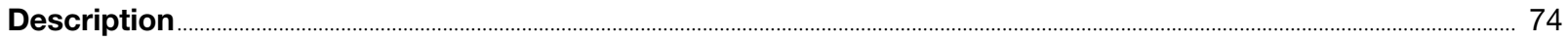

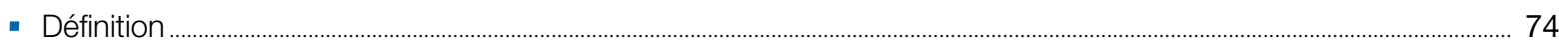

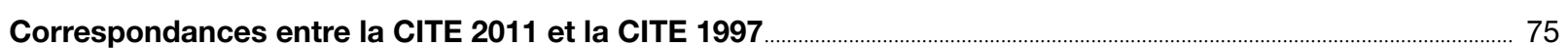


CHAPITRE 8 NIVEAU 5 DE LA CITE 2011 : ENSEIGNEMENT SUPÉRIEUR DE CYCLE COURT ....................... 77

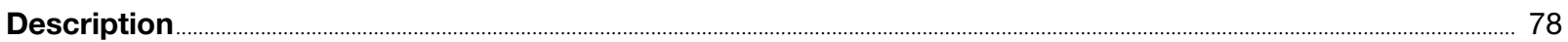

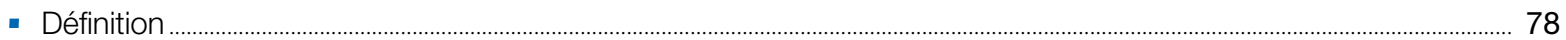

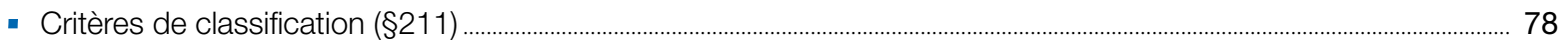

- Dimensions complémentaires ...................................................................................................................................................... 79

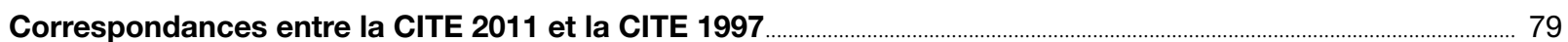

Directives pour la classification des programmes et pour les certifications reconnues correspondantes au niveau 5 de la CITE

Niveau d'éducation atteint au niveau 5 de la CITE

Exemples de programmes et de certifications classés au niveau 5 de la CITE ................................................... 80

CHAPITRE 9 NIVEAU 6 DE LA CITE 2011 : NIVEAU LICENCE OU ÉQUIVALENT ................................................... 85

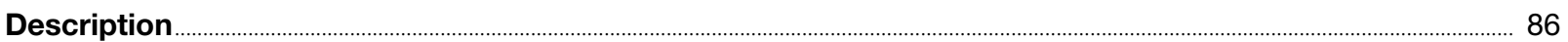

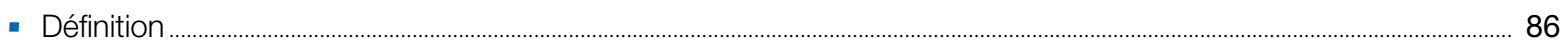

- Critères de classification $(\S 228)$.............................................................................................................................................................. 86

- Dimensions complémentaires ............................................................................................................................................................. 87

Correspondances entre la cite 2011 et la CITE 1997

Directives pour la classification des programmes et de leurs certifications reconnues correspondantes au niveau 6 de la CITE

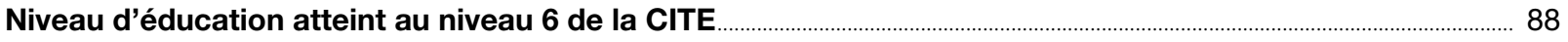

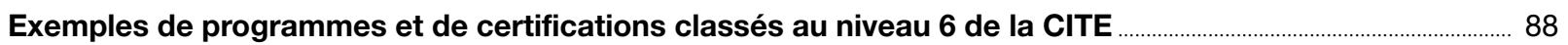

CHAPITRE 10 NIVEAU 7 DE LA CITE 2011 : NIVEAU MASTER OU ÉQUIVALENT ................................................. 93

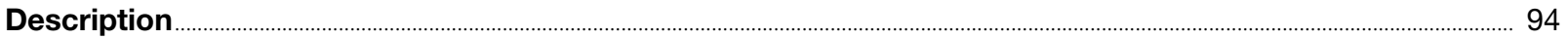

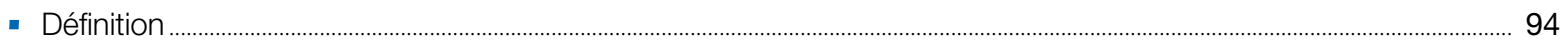

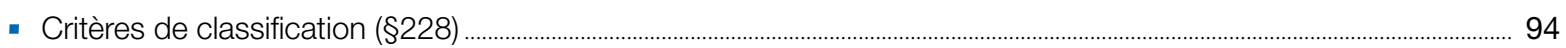

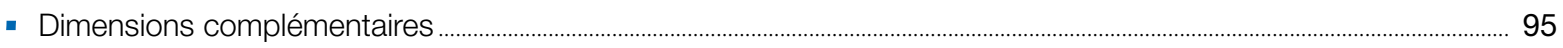

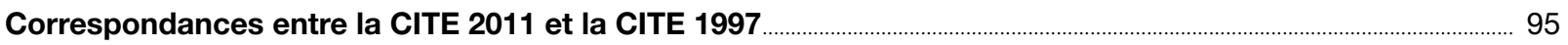

Directives pour la classification des programmes et de leurs certifications reconnues correspondantes au niveau 7 de la CITE

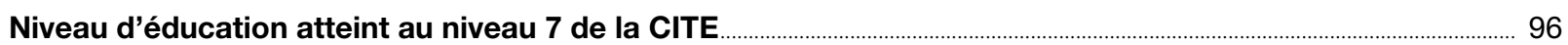

Exemples de programmes et de certifications classés au niveau 7 de la CITE ........................................................ 96

CHAPITRE 11 NIVEAU 8 DE LA CITE 2011 : NIVEAU DOCTORAT OU ÉQUIVALENT …....................................... 101

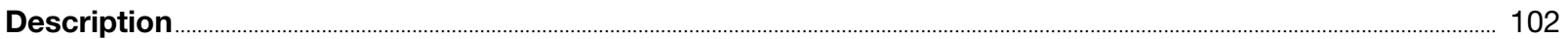

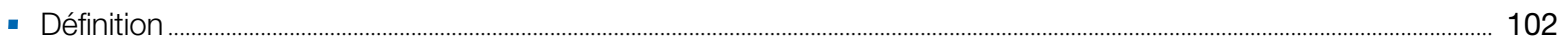

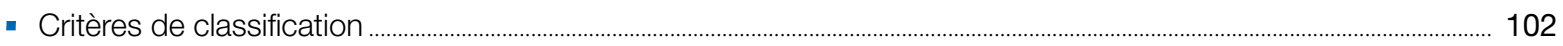

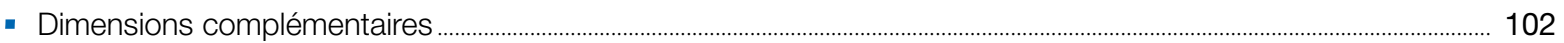

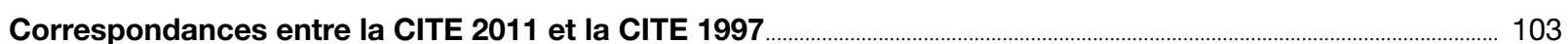

Directives pour la classification des programmes et de leurs certifications reconnues correspondantes au niveau 8 de la CITE

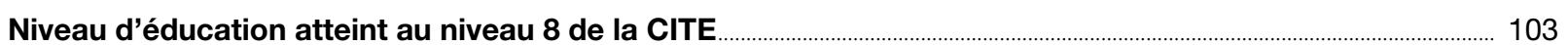

Exemples de programmes et de certifications classés au niveau 8 de la CITE .................................................... 104

ANNEX A TABLEAU RÉCAPITULATIF DES CODES ET DES CRITÈRES DE LA CITE 2011 ............................ 107

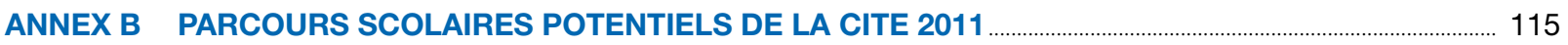

ANNEX C RÉFÉRENCE RAPIDE DES CODES DE LA CITE-P ET DE LA CITE-A DANS LA CITE 2011 ........ 117 



\section{Guide du lecteur}

Le Guide opérationnel CITE 2011 est destiné à assister les pays dans la classification des programmes éducatifs et des certifications nationaux dans leurs cartographies de la CITE. Le Guide aidera aussi les utilisateurs de statistiques internationales à comprendre quels types de programmes et de certification sont couverts par des indicateurs sur l'éducation. II fournit des directives et notes explicatives pour l'interprétation de la classification révisée et donne des exemples nationaux de programmes et de certifications correspondantes classés dans la CITE 2011.

Lorsque ce Guide cite directement le texte de la Classification Internationale Type de l'Éducation CITE 2011 (UNESCO-ISU, 2012), la référence au numéro de paragraphe correspondant dans la classification CITE 2011 précède la citation. Par exemple :

(§1) La Classification internationale type de l'Éducation (CITE) est la classification de référence permettant d'organiser les programmes éducatifs et les certifications correspondantes par niveau d'éducation et par domaines d'études.

Lorsque la référence à un numéro de paragraphe est mentionnée à la fin d'un texte dans le présent Guide, cela signifie que le concept est présenté dans la classification CITE 2011, mais que le texte du paragraphe auquel il est fait référence n'est pas cité au mot près. Par exemple :

Le développement éducatif de la petite enfance est destiné aux enfants de 0 à 2 ans et l'enseignement préprimaire aux enfants ayant entre 3 ans et l'âge d'entrée dans l'enseignement primaire. (§102)

La classification CITE 2011, qui contient les définitions du cadre officiel, est disponible dans les langues suivantes :

Arabe (www.uis.unesco.org/Education/Documents/isced-2011-ar.pdf)

Chinois (www.uis.unesco.org/Education/Documents/isced-2011-ch.pdf)

Anglais (www.uis.unesco.org/Education/Documents/isced-2011-en.pdf)

Français (www.uis.unesco.org/Education/Documents/isced-2011-fr.pdf)

Russe (www.uis.unesco.org/Education/Documents/isced-2011-ru.pdf)

Espagnol (www.uis.unesco.org/Education/Documents/isced-2011-sp.pdf)

La classification CITE 2011 contient également un glossaire étoffé des termes liés à la classification des activités éducatives.

- Pour consulter le glossaire de la CITE 2011, voir l'Annexe VI de la Classification CITE 2011.

Le Guide opérationnel est divisé en trois parties. La première partie présente un aperçu de la classification révisée. Elle met également en lumière les principales différences entre la CITE 2011 et la CITE 1997. Les deuxième et troisième parties fournissent des descriptions détaillées de chaque niveau de la CITE 2011.

Dans ces deux parties, chaque chapitre relatif à un niveau de la CITE contient des descriptions générales des caractéristiques du niveau, ainsi que des directives détaillées concernant l'affectation des programmes et des certifications correspondantes dans la CITE 2011, sur la base de critères spécifiques. Des exemples nationaux illustrent les codes à trois chiffres pour les classifications parallèles des programmes éducatifs (CITE-P) et des niveaux d'éducation atteints (CITE-A). Chaque chapitre détaille également les correspondances entre les niveaux respectifs de la CITE 2011 et de la CITE 1997.

L'Annexe A présente un tableau récapitulatif de tous les codes de la CITE 2011, avec les descriptions et les critères. L'Annexe B contient une représentation graphique des parcours scolaires potentiels de la CITE 2011. L'Annexe C fournit une référence croisée rapide entre les codes de la CITE-P et ceux de la CITE-A. 



\section{PARTIE I \\ APERÇU DE LA CITE 2011}

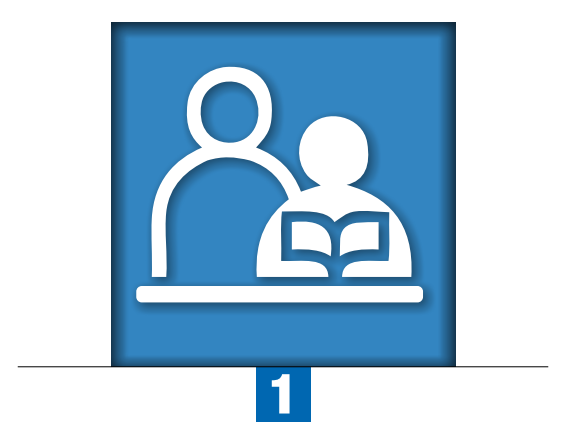

\section{Chapitre 1}

\section{Aperçu de la CITE 2011}

La Classification internationale type de l'Éducation (CITE) est la classification de référence permettant d'organiser les programmes éducatifs et les certifications correspondantes par niveau d'éducation et par domaines d'études. Les définitions et les concepts fondamentaux de la CITE ont été établis de manière à être internationalement valides et applicables à l'ensemble des systèmes éducatifs.

La CITE 2011 est la deuxième révision importante de cette classification (élaborée initialement dans les années 70 et révisée pour la première fois en 1997). Elle a été adoptée par la Conférence générale de l'UNESCO en novembre 2011.

La CITE 2011 présente une révision de la classification des niveaux des programmes éducatifs (CITE-P) de la CITE 1997 et une classification correspondante des niveaux d'éducation atteints (CITE-A) sur la base de certifications éducatives reconnues. En comparaison de la CITE 1997, qui comptait sept niveaux d'éducation, la CITE 2011 en compte maintenant neuf. 


\section{QU'EST-CE QUE LA CITE ET QUELLES SONT SES UNITÉS DE CLASSIFICATION ?}

$(\$ 1)^{1}$ La Classification internationale type de l'Éducation (CITE) est la classification de référence permettant d'organiser les programmes éducatifs et les certifications correspondantes par niveau d'éducation et par domaines d'études.

(§2) Les définitions et les concepts fondamentaux de la CITE ont été établis de manière à être internationalement valides et applicables à l'ensemble des systèmes éducatifs.

La CITE 2011 est la deuxième révision importante de cette classification (élaborée initialement dans les années 70 et révisée pour la première fois en 1997). La CITE 2011 a été adoptée par la Conférence générale de l'UNESCO en novembre 2011.

- Pour plus d'informations sur la CITE, voir la Section 1 de la Classification CITE 2011.

(\$10) Les unités fondamentales de classification de la CITE sont les programmes éducatifs nationaux (et infranationaux) et leurs certifications correspondantes reconnues.

(§11) Dans la CITE, un programme éducatif se définit comme une succession ou un ensemble cohérent d'activités éducatives ou de communication conçues et organisées en vue de réaliser des objectifs d'apprentissage préétablis ou un ensemble spécifique de tâches éducatives pendant une période durable.

(§20) Dans le contexte de la CITE, une certification éducative est la reconnaissance officielle, généralement sous la forme d'un document, qui vient sanctionner l'achèvement complet d'un programme éducatif ou du cycle d'un programme.

- Pour plus d'informations sur les unités de classification de la CITE, voir la Section 2 de la Classification CITE 2011.

\section{QUI EST RESPONSABLE DE LA CLASSIFICATION CITE ?}

(§91) L'Institut de statistique de I'UNESCO (ISU) est le responsable de la CITE et il assume donc la responsabilité du développement, de la maintenance, de la mise à jour et de la révision de cette classification de référence.

(§96) L'ISU projette de collaborer étroitement avec les pays et les organismes partenaires dans la collecte de données (y compris Eurostat et l'OCDE) afin de veiller à ce que les cartographies respectent la classification de la CITE et soient mises à jour en fonction des besoins.

- Pour plus d'informations sur la gouvernance de la CITE, voir la Section 8 de la Classification CITE 2011.

\section{QUE SONT LES CARTOGRAPHIES DE LA CITE ?}

(§8) Les cartographies de la CITE constituent un outil essentiel pour organiser l'information sur les systèmes éducatifs nationaux, leurs programmes et les certifications correspondantes afin d'assurer la comparabilité de l'information au niveau de la CITE et faciliter leur interprétation à des fins de statistiques internationales.

(§22) Dans la CITE, on classe en premier les programmes éducatifs puis les certifications. La cartographie de la CITE est l'outil permettant de montrer les relations entre les programmes éducatifs et les certifications.

Les cartographies de la CITE de chaque pays permettent de s'assurer que les comparaisons internationales sont plus transparentes et mieux comprises par les utilisateurs. Dès lors, l'information contextuelle présentée dans les cartographies est fondamentale. 
Ces informations comprennent, notamment, les dénominations nationales des programmes, les âges d'entrée et les durées théoriques des programmes, les critères d'admission minimaux et les certifications reçues après l'achèvement complet des programmes. Ces informations sont présentées parallèlement aux dimensions CITE pertinentes telles que le niveau de la CITE, l'orientation du programme, les sous-catégories d'achèvement de niveau et d'accès, et, aux niveaux licence et master, la position dans la structure nationale des diplômes.

La classification des programmes nationaux dans les niveaux de la CITE et les dimensions complémentaires peut ensuite être utilisée pour déterminer le code à trois chiffres CITE-P d'un programme et le code à trois chiffres correspondant de la CITE-A, qui porte sur la certification reçue à l'achèvement complet du programme.

Les cartographies de la CITE peuvent également inclure quelques notes avec des explications supplémentaires sur les programmes nationaux.

\section{QU'EST-CE QUI EST COUVERT OU NON PAR LA CLASSIFICATION CITE 2011 ?}

(§35) La CITE 2011 couvre les programmes éducatifs formel et non formel proposés à n'importe quel cycle de la vie d'un individu. Les certifications reconnues par les autorités nationales compétentes, quelle que soit la manière dont elles ont été obtenues (achèvement complet d'un programme éducatif formel, programme éducatif non formel ou activité d'apprentissage informelle) sont utilisées afin de mesurer le niveau d'éducation atteint. La CITE ne couvre pas les programmes de l'apprentissage informel, fortuit ou aléatoire ni les certifications non reconnues.

(§42) Dans la CITE 2011, on opère une distinction claire entre l'enseignement formel et l'enseignement non formel à des fins de statistiques. Actuellement, les activités internationales de collecte de données relatives à l'éducation (cartographies, enquêtes, recensements, etc.) se concentrent principalement sur l'enseignement formel.

Même si elles rentrent dans la portée de la CITE, les informations sur les programmes éducatifs non formels ne sont généralement pas incluses dans les collectes internationales de statistiques sur l'éducation. Toutefois, pour l'analyse du niveau d'éducation atteint, les certifications reconnues obtenues au terme de programmes éducatifs non formels sont habituellement prises en compte, si elles sont considérées comme équivalentes aux certifications obtenues grâce à l'enseignement formel.

- Pour plus d'informations sur la portée de l'éducation dans la CITE, voir la Section 4 de la Classification CITE 2011.

- Pour plus d'informations et des détails sur les questions relatives à l'enseignement non formel, voir la Section 4 et l'Annexe V de la Classification CITE 2011.

\section{QUELS SONT LES NIVEAUX D'ÉDUCATION DANS LA CLASSIFICATION CITE 2011 ?}

(§45) Les principales variables de classification de la CITE sont les niveaux d'éducation et les domaines d'étude.

Le présent Guide opérationnel se concentre sur la classification des programmes et des certifications correspondantes dans les niveaux de la CITE 2011.

La CITE 2011 présente neuf niveaux d'éducation, du niveau 0 au niveau 8 :

- CITE 0 : Éducation de la petite enfance

- CITE 1 : Enseignement primaire

- CITE 2 : Premier cycle de l'enseignement secondaire

- CITE 3 : Deuxième cycle de l'enseignement secondaire

- CITE 4 : Enseignement post-secondaire non-supérieur

- CITE 5 : Enseignement supérieur de cycle court

- CITE 6 : Niveau licence ou équivalent

- CITE 7 : Niveau master ou équivalent

- CITE 8 : Niveau doctorat ou équivalent 
(§47) Le niveau de la CITE reflète le degré de complexité et de spécialisation du contenu du programme éducatif, du stade élémentaire au plus complexe.

(§48) Plus le programme est avancé, plus le niveau d’éducation est élevé.

- Pour plus d'informations sur les niveaux de la CITE, voir les Sections 5 et 9 de la Classification CITE 2011.

\section{COMMENT LES PROGRAMMES ET LES CERTIFICATIONS CORRESPONDANTES SONT-ILS AFFECTÉS AUX NIVEAUX DE LA CITE 2011 ?}

(§50) La classification des programmes éducatifs par niveau vise à refléter leur contenu. Étant donné l'absence de mesures directes pour classifier le contenu éducatif, la CITE se fonde sur des critères d'approximation contribuant à assigner un programme éducatif donné à un niveau approprié de la CITE.

(§51) Ces critères d'approximation se répartissent en critères principaux et critères subsidiaires.

Par exemple, les mesures d'approximation susceptibles d'être utilisées pour la classification des programmes dans les niveaux sont la durée et la durée cumulée des programmes dans un ou plusieurs niveaux de la CITE. La CITE 2011 fournit quelques directives concernant une durée typique et des éventails de durée cumulative comme critères pour classer des programmes éducatifs par niveau. Ces critères sont résumés dans les Tableaux 1.1 et 1.2.

- Tableau 1.1 -

Durée par niveau de la CITE

\begin{tabular}{l|l} 
Niveau de la CITE & Durée typique [durée la plus commune]
\end{tabular}

\begin{tabular}{l|l}
0 & $\begin{array}{l}\text { Pas de critère de durée. Toutefois, un programme doit représenter au moins l'équivalent de } 2 \text { heures } \\
\text { par jour et de } 100 \text { jours par an d'activités éducatives pour être inclus. }\end{array}$ \\
\hline 1 & 4 à 7 ans [durée la plus fréquente $: 6$ ans] \\
\hline 2 & 2 à 5 ans [durée la plus fréquente $: 3$ ans] \\
\hline 3 & 2 à 5 ans [durée la plus fréquente $: 3$ ans] \\
\hline 4 & 6 mois à 2 ou 3 ans \\
\hline 5 & 2 à 3 ans \\
\hline 6 & $\begin{array}{l}3 \text { à } 4 \text { ans lorsque le programme suit directement un programme de niveau } 3 ; \text { ou } \\
1 \text { à } 2 \text { ans lorsque le programme suit un autre programme de niveau } 6\end{array}$ \\
\hline 7 & $\begin{array}{l}1 \text { à } 4 \text { ans lorsque le programme suit un programme de niveau } 6 ; \text { ou } \\
5 \text { à } 7 \text { ans lorsque le programme suit directement un programme de niveau } 3\end{array}$ \\
\hline 8 & Minimum 3 ans
\end{tabular}

- Tableau 1.2 .

Durée cumulée normale dans l'enseignement primaire et secondaire

\section{Niveau de la CITE Éventail [durée cumulée la plus commune]}

\begin{tabular}{l|l}
\hline 1 & 4 à 7 ans [durée la plus fréquente $: 6$ ans] \\
\hline $1+2$ & 8 à 11 ans [durée la plus fréquente $: 9$ ans] \\
\hline $1+2+3$ & 11 à 13 ans [durée la plus fréquente $: 12$ ans] \\
\hline
\end{tabular}

II existe d'autres critères d'approximation dans la CITE 2011, comme par exemple des exigences pour accéder au programme, le type de qualification de l'enseignant, l'organisation de l'instruction et l'objectif du programme (par exemple, préparer à l'entrée sur le marché du travail ou à l'accès à d'autres programmes éducatifs). 
Les prestataires de programmes éducatifs ne devraient pas être utilisés comme principal critère de classification des programmes. Le principal critère est la complexité et la spécialisation du contenu éducatif du programme et la manière dont le contenu se reflète dans les critères d'approximation (§52).

- Pour plus d'informations sur les critères de durée et de durée cumulée de la CITE, voir les Sections 5 et le Tableau 21 de la Classification CITE 2011.

(§49) La classification des programmes éducatifs sous forme de progression de niveaux vise à refléter la totalité des parcours éducatifs disponibles dans les systèmes éducatifs. La plupart des systèmes éducatifs proposent plusieurs parcours potentiels du niveau 0/1 au niveau 8 de la CITE.

- Pour consulter des parcours éducatifs potentiels par catégories de la CITE 2011, voir le Graphique 2 dans l'Annexe I de la Classification CITE 2011 et l'Annexe B du présent Guide opérationnel.

\section{COMMENT LES PROGRAMMES QUI COUVRENT PLUSIEURS NIVEAUX DE LA CITE, QUI SONT SÉQUENTIELS OU MODULAIRES, SONT-ILS CLASSÉS DANS LA CITE 2011 ?}

(§26) Lorsque l'on classe des programmes éducatifs nationaux par niveaux de la CITE, les points de transition entre les programmes nationaux et les points de sortie vers le marché du travail ne coïncident pas toujours avec les points de transition entre les niveaux de la CITE. Trois cas peuvent être identifiés : i) les programmes couvrant deux niveaux de la CITE ou plus ; ii) deux programmes successifs ou plus qui ensemble constituent un niveau de la CITE ; et iii) les programmes proposés sous forme de modules ou cours sans définition claire de succession.

Pour les programmes couvrant deux niveaux de la CITE ou plus, les années d'études, phases ou cycles remplissant les critères pour chaque niveau de la CITE doivent être affectés selon les différents niveaux. Les points de transition existant déjà dans les programmes peuvent aider à identifier la limite entre les niveaux de la CITE.

Deux programmes séquentiels ou plus constituant un niveau donné de la CITE doivent faire l'objet d'une attention particulière en ce qui concerne la soumission des statistiques. Par exemple, alors que les effectifs scolarisés seraient combinés pour tous les programmes du niveau, les données relatives aux entrants ne reprendraient que ceux du premier programme dans le niveau. De plus, les données relatives aux diplômés ne reprendraient que ceux qui ont terminé le dernier programme dans la succession des programmes du niveau (excepté quand les données sur les personnes ayant achevé avec succès des programmes menant à un achèvement partiel sont également collectées). (\$32)

Les programmes modulaires doivent également être évalués avec soin afin de s'assurer qu'ils remplissent tous les critères d'un programme éducatif dans la CITE 2011 et faire l'objet d'une attention particulière dans la soumission des données.

Pour plus d'informations sur les programmes couvrant plusieurs niveaux de la ClTE, séquentiels ou modulaires, voir la Section 3 de la Classification CITE 2011.

\section{PAR QUELLES DIMENSIONS COMPLÉMENTAIRES LES PROGRAMMES ET LES CERTIFICATIONS PEUVENT-ILS ÊTRE SUBDIVISÉS DANS LES NIVEAUX DE LA CITE 2011 ?}

(§45) Pour ce qui est des niveaux de la CITE, les programmes et les certifications sont subdivisés à l'aide de dimensions complémentaires. II s'agit de :

- L'orientation du programme ;

- L'achèvement du niveau de la CITE ;

- L'accès à des niveaux de la CITE plus élevés ; et

- La position dans la structure nationale des diplômes et des certifications. 
Pour les niveaux 2 à 5 de la CITE, il existe deux catégories d'orientation : le général et le professionnel. La CITE 2011 offre également la possibilité de codifier des catégories d'orientation académiques et professionnelles aux niveaux de l'enseignement supérieur (5 à 8). Toutefois, en l'absence de définitions internationalement convenues concernant les catégories d'orientation dans l'enseignement supérieur, la catégorie « non défini " peut être utilisée dans les statistiques internationales pour les niveaux 6 à 8 de la CITE.

La CITE 2011 introduit une nouvelle dimension complémentaire : achèvement d'un niveau de la CITE et accès à des niveaux d'éducation supérieurs. Au total, il existe quatre sous-catégories possibles de programmes (même si les quatre ne s'appliquent pas toujours à tous les niveaux) :

- Non achèvement de niveau - et donc pas d'accès direct à un niveau supérieur de la CITE (ce qui dans le cas du niveau 3 de la CITE est un accès aux niveaux 5, 6 ou 7) ;

- Achèvement partiel du niveau sans accès direct à un niveau supérieur de la CITE ;

- Achèvement du niveau sans accès direct à un niveau supérieur de la CITE ;

- Achèvement de niveau avec accès direct à un niveau supérieur de la CITE (ce qui dans le cas du niveau 3 de la CITE est un accès aux premiers programmes de l'enseignement supérieur aux niveaux 5, 6 ou 7).

Les programmes des niveaux 6 et 7 de la CITE peuvent être subdivisés en fonction de leur position dans la structure nationale des diplômes et des certifications (voir les chapitres correspondants pour de plus amples informations sur ces sous-catégories).

Les dimensions complémentaires ne s'appliquent pas toutes à tous les niveaux. Par ailleurs, le niveau 0 de la CITE est encore subdivisé selon le type de programme et le groupe d'âge ciblé : développement éducatif de la petite enfance et enseignement préprimaire.

- Pour plus d'informations sur les dimensions complémentaires de la CITE, voir la Section 5 de la Classification CITE 2011.

\section{DOMAINES D'ÉTUDES ET DE FORMATION}

Dans la CITE, les programmes et les certifications correspondantes peuvent être classés par domaine d'études et de formation, ainsi que par niveau. La révision 2011 de la CITE s'est concentrée sur les niveaux de la CITE et les dimensions complémentaires liées aux niveaux de la CITE.

À la suite de l'adoption de la CITE 2011, un processus d'analyse séparée et de consultation mondiale sur les domaines d'études de la CITE a été mené à bien. Les domaines d'études de la CITE ont été révisés et la Conférence générale de I'UNESCO a adopté la classification des Domaines d'études et de formation de la CITE 2013 (CITE-F 2013) en novembre 2013 lors de sa $37^{e}$ session.

La classification des Domaines d'études et de formation de la CITE 2013 (UNESCO-ISU, 2014) est disponible dans les langues suivantes :

Arabe (www.uis.unesco.org/Education/Documents/isced-fields-of-education-training-2013AR.pdf)

Chinois (www.uis.unesco.org/Education/Documents/isced-fields-of-education-training-2013CH.pdf)

Anglais (www.uis.unesco.org/Education/Documents/isced-fields-of-education-training-2013.pdf)

Français (www.uis.unesco.org/Education/Documents/isced-fields-of-education-training-2013FR.pdf)

Russe (www.uis.unesco.org/Education/Documents/isced-fields-of-education-training-2013RU.pdf)

Espagnol (www.uis.unesco.org/Education/Documents/isced-fields-of-education-training-2013ES.pdf)

\section{QUELS SONT LES MODĖLES DE CODIFICATION DE LA CITE 2011 ?}

(§89) La CITE se base sur des modèles de codification parallèles pour les programmes éducatifs (CITEProgrammes ou CITE-P) et les niveaux d'éducation atteints (CITE-Niveau atteint ou CITE-A). Des systèmes de code de trois chiffres sont utilisés pour codifier à la fois les programmes éducatifs et le niveau d'éducation atteint. 
II faut souligner que, s'il y a très souvent une correspondance entre le code CITE-P d'un programme et le code CITE-A résultant de l'achèvement complet du programme, les codes CITE-A ne peuvent pas toujours être déduits automatiquement des codes CITE-P. Par exemple, certains programmes éducatifs peuvent déboucher sur plusieurs certifications, chacune d'entre elle pouvant résulter en un niveau d'éducation atteint différent. Le programme sera classifié dans la CITE-P selon l'achèvement le plus élevé et la catégorie d'accès auxquels il mène, mais chaque certification qui en résulte sera classifiée dans la CITE-A en fonction du niveau d'éducation réellement atteint auquel elle est associée.

Les codes se terminant par " 1 » dans la CITE-P (programmes insuffisants pour un achèvement de niveau) n'existent pas dans la CITE-A puisque le niveau d'éducation atteint grâce à ces programmes est codifié selon le niveau de la CITE inférieur au programme d'études.

- Pour plus d'informations sur la codification de la CITE, voir les Tableaux 1-3 et les Annexes II et III de la Classification CITE 2011. Voir également les Annexes A et C du présent Guide opérationnel.

\section{COMPARAISON ENTRE LA CITE 2011 ET LA CITE 1997}

\section{Principales différences entre la CITE 2011 et la CITE 1997}

La CITE 2011 présente une révision de la classification des niveaux des programmes éducatifs (CITE-P) de la CITE 1997 et une classification correspondante des niveaux d'éducation atteints (CITE-A) sur la base de certifications éducatives reconnues.

La CITE 2011 propose des définitions améliorées de l'enseignement formel et non formel, des activités éducatives et des programmes. Elle comprend également un glossaire plus étoffé offrant aux utilisateurs une meilleure compréhension des termes spécifiques de la CITE.

Comparée à la CITE 1997, qui comptait sept niveaux d'éducation, la CITE 2011 en compte maintenant neuf. L'enseignement supérieur a été restructuré pour prendre en compte les changements qui l'ont affecté, tels que la structure de Bologne, et il compte maintenant quatre niveaux d'éducation, contre deux auparavant dans la CITE 1997. Les programmes que l'on classifiait auparavant dans le niveau 5 de la CITE 1997 sont dorénavant affectés aux niveaux 5 , 6 ou 7 de la CITE 2011. De même, le niveau 0 de la CITE a été étendu pour inclure une nouvelle catégorie couvrant des programmes de développement éducatif de la petite enfance destinés aux enfants de moins de 3 ans. Chaque niveau d'éducation de la CITE a été défini avec plus de clarté, ce qui pourrait occasionner des changements de classification pour des programmes qui se trouvaient auparavant à la limite entre des niveaux de la CITE (par exemple, entre les niveaux 3 et 4 de la CITE).

La CITE 2011 comprend deux classifications parallèles, la CITE-P et la CITE-A, chacune représentée par un modèle de codification par trois chiffres séparé mais similaire.

Les dimensions complémentaires des niveaux de la CITE ont également été révisées. II n'existe aujourd'hui que deux catégories d'orientation : l'enseignement général et l'enseignement professionnel. Les programmes classés au préalable dans la catégorie préprofessionnelle (CITE 1997) ne permettent pas l'obtention de certifications pertinentes pour le marché du travail et de ce fait sont maintenant classés pour la plupart dans l'enseignement général (\$278). La CITE 2011 offre également des codes pour des orientations « académiques » et " professionnelles » au niveau de l'enseignement supérieur. Toutefois, comme un accord international doit encore être atteint sur leurs définitions, le code « orientation non définie " peut également être utilisé aux niveaux 6 à 8 de la CITE.

La CITE 1997 distinguait deux sous-catégories concernant l'accès à des niveaux plus élevés de la CITE selon le type d'enseignement auquel la certification obtenue donnait accès, alors que la CITE 2011 n'identifie qu'un groupe de programmes donnant accès à des niveaux plus élevés de la CITE. La sous-catégorie " achèvement de niveau avec accès à des niveaux plus élevés de la CITE 2011 » correspond aux catégories A et B combinées de la CITE 1997. La CITE 2011 subdivise encore les programmes qui ne permettent pas d'accéder à des niveaux supérieurs de la CITE dans les sous-catégories " sans achèvement de niveau ", " achèvement partiel de niveau » et « achèvement de niveau ». Ces trois sous-catégories correspondent à la catégorie C de la CITE 1997. (§278)

De plus, alors que la position des programmes d'enseignement supérieur dans la structure nationale des diplômes était mentionnée dans la CITE 1997, une codification spécifique pour cette dimension a été introduite dans la CITE 2011 pour les niveaux 6 et 7 (niveaux licence ou master ou équivalents respectivement). 
Tableaux de correspondance entre les révisions de la CITE

Le Tableau 1.3 illustre les correspondances entre les niveaux de la CITE 2011 et de la CITE 1997.

- Tableau 1.3 -

Comparaison des niveaux d'éducation entre la CITE 2011 et la CITE 1997

\begin{tabular}{|c|c|c|c|}
\hline \multicolumn{2}{|c|}{ CITE 2011} & \multicolumn{2}{|c|}{ CITE 1997} \\
\hline 01 & Développement éducatif de la petite enfance & & - \\
\hline 02 & Enseignement préprimaire & 0 & Enseignement préprimaire \\
\hline 1 & Enseignement primaire & 1 & $\begin{array}{l}\text { Enseignement primaire ou premier cycle de } \\
\text { l'éducation de base }\end{array}$ \\
\hline 2 & Premier cycle de l'enseignement secondaire & 2 & $\begin{array}{l}\text { Premier cycle de l'enseignement secondaire ou } \\
\text { deuxième cycle de l'éducation de base }\end{array}$ \\
\hline 3 & Deuxième cycle de l'enseignement secondaire & 3 & Deuxième cycle de l'enseignement secondaire \\
\hline 4 & Enseignement post-secondaire non-supérieur & 4 & Enseignement post-secondaire non-supérieur \\
\hline 5 & Enseignement supérieur de cycle court & \multirow[t]{3}{*}{5} & \multirow{3}{*}{$\begin{array}{l}\text { Premier cycle de l'enseignement supérieur (ne } \\
\text { menant pas directement à une certification de } \\
\text { chercheur hautement qualifié) }(5 A, 5 B)\end{array}$} \\
\hline 6 & Niveau licence ou équivalent & & \\
\hline 7 & Niveau master ou équivalent & & \\
\hline 8 & Niveau doctorat ou équivalent & 6 & $\begin{array}{l}\text { Second cycle de l'enseignement supérieur (menant à } \\
\text { une certification de chercheur hautement qualifié) }\end{array}$ \\
\hline
\end{tabular}

Le Tableau 1.4 illustre les correspondances entre les dimensions complémentaires de la CITE 2011 et de la CITE 1997.

- Tableau 1.4 -

Comparaison des dimensions complémentaires entre la CITE 2011 et la CITE 1997

\begin{tabular}{|c|c|c|c|c|c|}
\hline \multicolumn{3}{|c|}{ CITE 2011} & \multicolumn{3}{|c|}{ CITE 1997} \\
\hline \multicolumn{2}{|c|}{$\begin{array}{l}\text { Orientation } \\
\text { (deuxième chiffre du code) }\end{array}$} & \multirow{4}{*}{$\begin{array}{l}\text { Niveaux } \\
\text { concernés } \\
2 \text { à } 5\end{array}$} & \multicolumn{2}{|c|}{ Orientation } & $\begin{array}{l}\text { Niveaux } \\
\text { concernés }\end{array}$ \\
\hline \multirow[t]{2}{*}{4} & \multirow[t]{2}{*}{ Enseignement général } & & $\mathrm{G}$ & Enseignement général & \multirow[t]{3}{*}{2 à 4} \\
\hline & & & $\mathrm{P}$ & $\begin{array}{l}\text { Enseignement préprofessionnel ou } \\
\text { prétechnique }\end{array}$ & \\
\hline 5 & Enseignement professionnel & & V & Enseignement professionnel ou technique & \\
\hline 4 & Enseignement académique* & 5 à 8 & & - & \\
\hline 5 & Enseignement professionnel ${ }^{\star}$ & 5 à 8 & & - & \\
\hline 6 & Orientation non définie* & 6 à 8 & & - & \\
\hline \multicolumn{2}{|c|}{$\begin{array}{l}\text { Achèvement et accès } \\
\text { (troisième chiffre du code CITE-P) }\end{array}$} & $\begin{array}{l}\text { Niveaux } \\
\text { concernés }\end{array}$ & \multicolumn{2}{|c|}{ Destination } & $\begin{array}{l}\text { Niveaux } \\
\text { concernés }\end{array}$ \\
\hline \multirow[t]{5}{*}{4} & \multirow[t]{5}{*}{$\begin{array}{l}\text { Suffisant pour l'achèvement du } \\
\text { niveau avec un accès direct au niveau } \\
\text { supérieur de la CITE }\end{array}$} & \multirow[t]{5}{*}{2 à 5,8} & \multirow[t]{2}{*}{ A } & $\begin{array}{l}\text { Programmes conçus pour mener à des } \\
\text { programmes « } A \text { » au niveau supérieur } \\
\text { de la CITE (accès direct à des études de } \\
\text { niveau doctorat) }\end{array}$ & 2 à 4 \\
\hline & & & & $\begin{array}{l}\text { Programmes fondés sur la théorie } \\
\text { au premier niveau de l'enseignement } \\
\text { supérieur }\end{array}$ & 5 \\
\hline & & & \multirow[t]{3}{*}{$\mathrm{B}$} & $\begin{array}{l}\text { Programmes conçus pour mener à des } \\
\text { programmes «B » au niveau supérieur } \\
\text { de la CITE (parcours vers le marché du } \\
\text { travail) }\end{array}$ & $2,3,\left(4^{\star \star}\right)$ \\
\hline & & & & $\begin{array}{l}\text { Programmes conçus pour mener au } \\
\text { marché du travail }\end{array}$ & $4^{\star \star}$ \\
\hline & & & & $\begin{array}{l}\text { Programmes orientés vers la pratique/ } \\
\text { professionnellement spécifiques au } \\
\text { premier niveau de l'enseignement } \\
\text { supérieur }\end{array}$ & 5 \\
\hline
\end{tabular}


- Tableau 1.4 (suite) -

Comparaison des dimensions complémentaires entre la CITE 2011 et la CITE 1997

\begin{tabular}{|c|c|c|c|c|c|}
\hline \multicolumn{3}{|c|}{ CITE 2011} & \multicolumn{3}{|c|}{ CITE 1997} \\
\hline \multicolumn{2}{|c|}{$\begin{array}{l}\text { Achèvement et accès } \\
\text { (troisième chiffre du code ClTE-P) }\end{array}$} & \multirow{2}{*}{$\begin{array}{l}\text { Niveaux } \\
\text { concernés } \\
2 \text { à } 4\end{array}$} & \multicolumn{2}{|c|}{ Destination } & $\begin{array}{l}\text { Niveaux } \\
\text { concernés }\end{array}$ \\
\hline 3 & $\begin{array}{l}\text { Suffisant pour l'achèvement du niveau } \\
\text { sans accès direct au niveau supérieur } \\
\text { de la CITE }\end{array}$ & & \multirow[t]{3}{*}{ C } & \multirow[t]{3}{*}{$\begin{array}{l}\text { Programmes conçus pour mener au } \\
\text { marché du travail, sans accès direct au } \\
\text { niveau supérieur de la CITE }\end{array}$} & \multirow[t]{3}{*}{$2,3,\left(4^{\star \star}\right)$} \\
\hline 2 & $\begin{array}{l}\text { Achèvement partiel du niveau sans } \\
\text { accès direct au niveau supérieur de la } \\
\text { CITE }\end{array}$ & 2,3 & & & \\
\hline 1 & $\begin{array}{l}\text { Insuffisant pour l'achèvement du niveau } \\
\text { sans accès direct au niveau supérieur } \\
\text { de la CITE }\end{array}$ & 2 à 8 & & & \\
\hline \multicolumn{2}{|c|}{$\begin{array}{l}\text { Position dans la structure nationale des } \\
\text { diplômes (troisième chiffre du code ClTE-P) }\end{array}$} & $\begin{array}{l}\text { Niveaux } \\
\text { concernés }\end{array}$ & & & \\
\hline 5 & Premier diplôme & 6 & - & - & - \\
\hline 6 & Premier diplôme long & 6,7 & - & - & - \\
\hline 7 & $\begin{array}{l}\text { Deuxième diplôme ou diplôme } \\
\text { supplémentaire après un programme } \\
\text { de licence ou équivalent }\end{array}$ & 6,7 & - & - & - \\
\hline 8 & $\begin{array}{l}\text { Deuxième diplôme ou diplôme } \\
\text { supplémentaire après un programme } \\
\text { de master ou équivalent }\end{array}$ & 7 & - & - & - \\
\hline
\end{tabular}

* Étant donné l'absence de définitions internationalement convenues concernant les orientations académique et professionnelle dans l'enseignement supérieur, les termes 'général' ou 'professionnel' seront utilisés pour les niveaux 2 à 5 de la CITE 2011, et 'orientation non définie' peut être utilisé pour les niveaux 6 à 8 de la CITE 2011 .

** La destination C de la CITE 1997 a été introduite au niveau 4 de la CITE pour les pays UOE (collecte de données UNESCO-OCDE-Eurostat). Pour les autres pays, seules les destinations A et B s'appliquaient au niveau 4 de la CITE 1997.

- Pour plus de détails sur les correspondances entre les niveaux de la CITE 2011 et de la CITE 1997, voir Section 10 et Tableaux 19, 20 et 21 de la Classification CITE 2011.

\section{Note}

1. Les numéros de paragraphe constituent des références à la classification CITE 2011. Pour plus de détails, veuillez consulter le Guide du lecteur. 



\section{PARTIE II \\ NIVEAUX 0 À 4 DE LA CITE 2011 : \\ DE L'ÉDUCATION DE LA PETITE ENFANCE \\ À L'ENSEIGNEMENT POST-SECONDAIRE NON-SUPÉRIEUR}

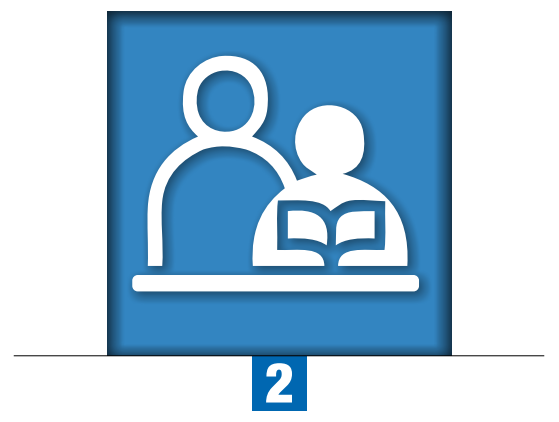

\section{Chapitre 2}

\section{Niveau 0 de la CITE 2011:}

\section{Éducation de la petite enfance}

Le niveau 0 de la CITE regroupe les programmes de la petite enfance qui possèdent une composante éducative volontaire. Les programmes de niveau 0 de la CITE ciblent les enfants dont l'âge est inférieur à celui d'entrée dans l'enseignement primaire (niveau 1 de la CITE). Ces programmes ont pour but de développer les aptitudes sociales et émotionnelles nécessaires pour participer à la vie scolaire et sociale.

Les programmes classés au niveau 0 de la CITE peuvent être désignés de plusieurs manières, par exemple : l'éducation et le développement de la petite enfance, école maternelle, école préprimaire, pre-school ou educación inicial. En ce qui concerne les programmes offerts en crèches, nurseries ou guarderías, il importe de s'assurer qu'ils respectent les critères de classification du niveau 0 de la CITE. En vue de la comparabilité internationale, l'expression « éducation de la petite enfance " est utilisée afin de qualifier le niveau 0 de la CITE. 


\section{DESCRIPTION}

\section{Définition}

(\$100)느 Les programmes du niveau 0 de la CITE ont généralement une approche holistique et visent essentiellement à encourager le développement cognitif, linguistique, physique, social et émotionnel précoces des jeunes enfants et à les préparer à un enseignement organisé en dehors du contexte familial. Le niveau 0 de la CITE regroupe les programmes de la petite enfance qui possèdent une composante éducative volontaire.

(§101) À ce niveau, les programmes ne sont pas nécessairement très structurés mais ils sont conçus pour offrir un ensemble organisé et intentionnel d'activités d'apprentissage dans un environnement sûr. lls permettent aux enfants d'apprendre à travers l'interaction avec d'autres enfants, sous la supervision du personnel ou d'éducateurs, généralement par le biais d'activités créatives et ludiques.

(§102) Les programmes de niveau 0 de la CITE ciblent les enfants dont l'âge est inférieur à celui d'entrée dans le niveau 1 de la CITE. II existe deux catégories de programmes de niveau 0 de la CITE : le développement éducatif de la petite enfance et l'enseignement préprimaire. Le premier se caractérise par un contenu éducatif développé pour des enfants plus jeunes (0 à 2 ans) alors que la seconde est généralement prévue pour des enfants ayant entre 3 ans et l'âge d'entrée dans l'enseignement primaire.

\section{Critères de classification}

\section{Critères principaux}

a. Propriétés éducatives du programme

(§105) Les propriétés éducatives du développement éducatif de la petite enfance [CITE-P 010] sont caractérisées par un environnement d'apprentissage qui est visuellement stimulant et linguistiquement riche. Ces programmes encouragent l'expression personnelle tout en mettant l'accent sur l'acquisition du langage et son utilisation pour une communication pertinente. Ils offrent des opportunités de jeu actif afin que les enfants puissent travailler leur coordination et leur habileté motrice, sous supervision et à travers l'interaction avec le personnel.

(§106) Les propriétés éducatives de l'enseignement préprimaire [CITE-P 020] sont caractérisées par une interaction avec leurs pairs et les éducateurs à travers laquelle les enfants améliorent leur utilisation du langage et leurs aptitudes sociales, commencent à développer des compétences de raisonnement logique et parlent tout en réfléchissant. On les initie également à des concepts alphabétiques et mathématiques et on les encourage à explorer leur environnement. Des activités supervisées de motricité (c'est-à-dire de l'exercice physique au travers de jeux et autres activités) et de jeu peuvent être utilisées comme opportunités d'apprentissage pour favoriser les interactions sociales entre pairs et développer aptitudes, autonomie et préparation à l'école.

b. Contexte institutionnel

(\$107) Les programmes du niveau 0 de la CITE se déroulent habituellement à l'école ou dans une autre institution pour un groupe d'enfants (par exemple dans un centre, une communauté ou à domicile). Le niveau 0 de la CITE exclut les services fournis exclusivement dans le cadre familial qui peuvent être intentionnels mais ne sont pas organisés en " programme » (par exemple, l'apprentissage informel donné aux enfants par leurs parents, d'autres membres de la famille ou des amis n'est pas inclus au niveau 0 de la CITE).

c. Âge général des enfants ciblés par le programme

(§108) Dans la CITE 0, les programmes de développement éducatif de la petite enfance sont destinés à des enfants âgés de 0 à 2 ans et les programmes d'enseignement préprimaire sont destinés à des enfants âgés de 3 ans jusqu'à l'âge d'entrée à la CITE 1. La limite d'âge supérieure de la catégorie enseignement préprimaire dépend de l'âge théorique d'entrée dans le niveau 1 de la CITE, c'est-à-dire l'enseignement primaire. 


\section{d. Durée/intensité du programme}

(\$110) La CITE recommande la durée et l'intensité minimales suivantes afin d'améliorer la comparabilité internationale : les programmes éducatifs doivent représenter au moins l'équivalent de 2 heures par jour et 100 jours par an d'activités éducatives pour être pris en compte dans la CITE.

\section{Critères subsidiaires}

a. Qualifications du personnel enseignant

(§111) Le cas échéant, les certifications pédagogiques exigées du personnel enseignant peuvent fournir un bon critère de substitution pour un programme éducatif dans les systèmes éducatifs où cette exigence existe. Ce critère permet de distinguer l'éducation de la petite enfance de la garderie pour laquelle aucune certification explicitement pédagogique pour le personnel n'est exigée.

\section{b. Existence d'un cadre de réglementation}

(§112) Le cas échéant, l'existence d’un cadre de référence ou de réglementation défini ou reconnu par les autorités nationales compétentes (par exemple le ministère de l'Éducation, une autre institution ou un ministère compétents) peut constituer un bon critère de substitution pour un programme éducatif. Ce cadre doit se caractériser par des directives, des normes ou des instructions décrivant les opportunités d'apprentissage offertes aux jeunes enfants.

c. Ne fait généralement pas partie de la scolarité obligatoire

(§113) Dans les systèmes éducatifs avec scolarité obligatoire, les programmes éducatifs non obligatoires conçus pour se dérouler avant le commencement de la scolarité obligatoire et qui répondent aux critères ci-dessus sont classés au niveau 0 de la CITE. En outre, le premier cycle ou phase de scolarité obligatoire peut aussi être classé au niveau 0 de la CITE dans certains pays s'il répond aux critères de ce niveau. Ainsi, le commencement de la scolarité obligatoire n'est pas un critère suffisant pour distinguer les programmes du niveau 0 de la CITE des programmes du niveau 1 même si cela peut être le cas dans certains systèmes éducatifs.

\section{Dimensions complémentaires}

(§116) Une dimension distingue les programmes éducatifs du niveau 0 de la CITE :

- Groupe d’âge ciblé

Les programmes de niveau 0 de la CITE ciblent les enfants dont l'âge est inférieur à celui d'entrée dans le niveau 1 de la CITE. Le développement éducatif de la petite enfance est destiné aux enfants de 0 à 2 ans et l'enseignement préprimaire aux enfants ayant entre 3 ans et l'âge d'entrée dans l'enseignement primaire. (§102)

\section{CORRESPONDANCES ENTRE LA CITE 2011 ET LA CITE 1997}

§275) Dans la CITE 2011, le niveau 0 couvre l'éducation de la petite enfance pour tous les âges, y compris les enfants très jeunes. Les programmes sont subdivisés en deux catégories selon la complexité du contenu éducatif : développement éducatif de la petite enfance (code 010) et enseignement préprimaire (code 020). Les programmes de développement éducatif de la petite enfance (code 010) ciblent généralement les enfants de moins de 3 ans. Elle est introduite comme nouvelle catégorie pour la première fois dans la CITE 2011 et n'est pas couverte par la CITE 1997. L'enseignement préprimaire (code 020) correspond exactement au niveau 0 de la CITE 1997. 


\section{DIRECTIVES POUR LA CLASSIFICATION DES PROGRAMMES ET DE LEURS CERTIFICATIONS RECONNUES CORRESPONDANTES AU NIVEAU 0 DE LA CITE}

Le niveau 0 de la CITE regroupe les programmes de la petite enfance qui possèdent une composante éducative volontaire. Les programmes de niveau 0 de la CITE ciblent les enfants dont l'âge est inférieur à celui d'entrée dans le niveau 1 de la CITE. Ces programmes ont pour but de développer les aptitudes cognitives, physiques, sociales et émotionnelles nécessaires pour participer à la vie scolaire et sociale. $(\$ 100,102)$

Les programmes du niveau 0 de la CITE se différencient souvent par l'âge. II existe deux catégories de programmes de niveau 0 de la CITE : la CITE 010 - développement éducatif de la petite enfance et la CITE 020 - enseignement préprimaire. La CITE 010 se caractérise par un contenu éducatif volontaire développé pour des enfants plus jeunes (ayant habituellement entre 0 et 2 ans) (Exemples Groupe 1), alors que la CITE 020 est généralement prévue pour des enfants ayant entre 3 ans et l'âge d'entrée dans l'enseignement primaire (niveau 1 de la CITE) (\$108) (Exemples Groupe 2).

Certains programmes du niveau 0 de la CITE couvrent les deux catégories de ce niveau (ces programmes éducatifs ciblent des enfants très jeunes jusqu'à l'âge d'entrée dans le niveau 1 de la CITE). La classification de ces programmes intégrés d'éducation de la petite enfance doit faire l'objet d'une attention particulière. Les programmes divisés en années d'études, étapes ou cycles doivent être classés dans chacune des deux catégories en fonction des propriétés éducatives du programme (voir critères principaux). Lorsqu'il n'y a pas de subdivision du programme, la classification dans les deux catégories doit dépendre de l'âge des participants. (\$109) Dans le cadre de la soumission des données, les données relatives aux enfants de moins de 3 ans présents dans ces programmes intégrés doivent être rapportées comme CITE 010 ; les données relatives aux enfants de 3 ans et plus doivent être rapportées comme CITE 020 (Exemples Groupe 3).

II faut être particulièrement attentif dans la classification de programmes à domicile. Pour des exemples de programmes destinés à la petite enfance se déroulant à domicile devant être considérés ou non comme appartenant au niveau 0 de la CITE, voir Exemples Groupe 4 et Exemples Groupe 5 respectivement.

De même, les programmes qui procurent uniquement des services de garderie (surveillance, nutrition et santé) ne sont pas couverts par le niveau 0 de la CITE. Lorsqu'il existe à la fois des programmes éducatifs et non éducatifs dans un pays et qu'il est possible de s'engager dans chaque type indépendamment, seuls les programmes éducatifs doivent être classés au niveau 0 de la CITE. Néanmoins, il faut noter qu'il est acceptable d'envisager la classification de programmes de la petite enfance réunissant à la fois des composantes éducatives et de service de garderie dans le niveau 0 de la CITE avec une part non éducative supérieure à la part éducative, pour autant que la part éducative remplisse les autres critères décrits ci-dessus, y compris les critères d'intensité minimale et de durée.

Outre un accent mis sur le développement de l'enfant ainsi que sur la composante éducative volontaire, un des facteurs clés des programmes du niveau 0 de la CITE est la continuité, en termes d'intensité et de durée, des activités éducatives prévues. Ces points différencient les programmes du niveau 0 de la CITE d'autres programmes de la petite enfance, tels que les services de garderie et les soins occasionnels, le soir ou pendant les vacances. On s'attend à ce que les enfants engagés dans un programme du niveau 0 de la CITE bénéficient d'une expérience d'apprentissage durable caractérisée par la continuité et la durée d'activités éducatives volontaires. Lorsque les autres critères principaux (et le cas échéant les critères subsidiaires) sont remplis, la composante éducative volontaire du programme doit représenter au moins l'équivalent de 2 heures par jour en intensité et 100 jours par an en durée, pour être prise en compte dans le niveau 0 de la CITE. À noter : il s'agit là d'une intensité et d'une durée minimales; la part d'éducation volontaire des programmes et les programmes eux-mêmes peuvent les dépasser.

II faut veiller à distinguer l'intensité/la durée des composantes d'éducation volontaire des programmes du niveau 0 de la CITE, l'intensité/la durée attendues de la fréquentation des enfants inscrits, et les heures d'ouverture des établissements où les programmes se déroulent, car ils ne sont pas nécessairement identiques.

Si un programme remplit les principaux critères pour le niveau 0 de la CITE et qu'il est prévu que les enfants inscrits dans le programme mènent des activités éducatives volontaires caractérisées par l'intensité/la durée spécifiées, le programme dans son ensemble peut être considéré comme un programme éducatif dans la CITE 2011. Cela inclut les cas où un programme comprend des activités supplémentaires qui n'ont pas spécifiquement des visées éducatives, pour autant que le programme satisfait aux critères d'intensité/de durée minimales des activités éducatives volontaires.

Si des informations exactes sur l'intensité et la durée des composantes éducatives volontaires d'un programme ne sont pas disponibles, il ne faut pas utiliser les heures d'ouverture d'un établissement ou les heures d'exécution du programme comme 
approximation pour déterminer si le programme doit être inclus dans le niveau 0 de la CITE. Dans ce cas, il faut utiliser une estimation de l'intensité/de la durée typiques ou envisagées des propriétés éducatives volontaires du programme.

Les programmes doivent être exclus du niveau 0 de la CITE si la fréquentation attendue des enfants inscrits est inférieure aux critères minimaux d'intensité et de durée, quels que soient le nombre d'heures ou de jours où le programme est disponible ou l'intensité/la durée du contenu éducatif volontaire (Exemples Groupe 5).

Voici quelques exemples typiques de programmes à exclure du niveau 0 de la CITE car la fréquentation attendue des enfants inscrits ne satisfait pas aux critères minimaux d'intensité et de durée :

- les programmes où la fréquentation peut être sur mesure ou de type prise en charge libre où les enfants ne connaîtront pas une continuité dans les opportunités d'apprentissage structuré ;

- les programmes de courte durée, comme une prise en charge en vacances, qui peuvent bénéficier d'une composante éducative mais non d'une période suivie d'opportunités d'instruction ou d'apprentissage ; et

- les programmes avec des propriétés éducatives volontaires mais sans niveau minimal de fréquentation, de telle sorte que les parents sont libres de choisir l'intensité et la durée de la fréquentation de leur enfant mais qu'elles ne correspondent pas aux critères minimaux à atteindre dans le cadre du niveau 0 de la CITE.

Le commencement de la scolarité obligatoire n'est pas un critère suffisant pour distinguer les programmes du niveau 0 de la CITE des programmes du niveau 1 même si cela peut être le cas dans certains systèmes éducatifs.

Le niveau 0 de la CITE inclut des programmes pour des enfants ayant des besoins éducatifs spéciaux correspondant aux critères pour ce niveau, quel que soit leur âge (\$117). L'instruction organisée pour les enfants ayant des besoins éducatifs spéciaux doit être incluse dans ce niveau si les participants ont un âge similaire à celui d'autres élèves suivant un programme de développement éducatif de la petite enfance ou d'enseignement préprimaire, ou si le contenu éducatif est d'un niveau significativement inférieur à celui des premières années de l'enseignement primaire (même si les élèves n'ont pas l'âge typique du niveau 0 de la CITE). Cela peut notamment inclure l'éducation fournie dans les hôpitaux, des établissements d'enseignement spécial ou des centres de formation.

\section{Programmes couvrant les niveaux 0 et 1 de la CITE}

La classification des programmes éducatifs couvrant les niveaux 0 et 1 de la CITE nécessite une attention particulière. Dans les systèmes éducatifs où une partie de l'éducation nationale de la petite enfance est comprise dans l'enseignement primaire, seuls les années d'études, phases ou cycles correspondant aux critères des niveaux respectifs (niveau 0 ou 1 de la CITE) doivent être classés dans ces niveaux. (\$114)

Si l'utilisation des critères de classification ne permet pas d'établir une distinction claire entre les niveaux 0 et 1 de la CITE, il est recommandé de procéder comme suit : (\$115)

i) pour les programmes couvrant les niveaux 0 et 1 de la CITE organisés sous la forme de cycles, la fin du cycle la plus proche de l'âge de 6 ans doit être considérée comme le point de transition entre les niveaux 0 et 1 de la CITE ; et

ii) pour les programmes couvrant les niveaux 0 et 1 de la CITE qui ne sont pas répartis en cycles, les années d'études destinées aux enfants de moins de six ans doivent être classées dans le niveau 0 de la CITE alors que les autres années d'études devraient être classées dans le niveau 1 de la CITE.

\section{NIVEAU D'ÉDUCATION ATTEINT AU NIVEAU 0 DE LA CITE}

(§119) Dans le but de classer le niveau d'éducation atteint, le niveau 0 (inférieur à l'enseignement primaire) est utilisé pour les enfants qui :

- n'ont jamais participé à un programme éducatif (CITE-A 010) ;

- ont eu une participation limitée à des programmes d'éducation de la petite enfance (CITE-A 020) ; ou

- ont eu une scolarisation limitée au primaire mais n'ont pas achevé complètement le niveau 1 de la CITE (avec ou sans fréquentation du niveau 0 de la CITE) (CITE-A 030).

Le code « 020 » de la CITE-A inclut les enfants ayant participé à un programme de développement éducatif de la petite enfance ou d'enseignement préprimaire, ou les deux. 


\section{EXEMPLES DE PROGRAMMES ET DE CERTIFICATIONS CLASSÉS AU NIVEAU O DE LA CITE}

(§103) Les programmes classés au niveau 0 de la CITE peuvent être désignés de plusieurs manières, par exemple : l'éducation et le développement de la petite enfance, école maternelle, école préprimaire, pre-school ou educación inicial. En ce qui concerne les programmes offerts en crèches, nurseries ou guarderías, il importe de s'assurer qu'ils respectent les critères de classification du niveau 0 de la CITE spécifiés. En vue de la comparabilité internationale, l'expression « éducation de la petite enfance » est utilisée afin de qualifier le niveau 0 de la CITE.

\section{Exemples Groupe 1 : \\ Programmes de développement éducatif de la petite enfance [CITE-P : 010]}

Bahrein - (Garderies). Ce programme de développement éducatif de la petite enfance est destiné aux enfants de moins de 3 ans. II est supervisé par le Ministère du Développement social et est considéré comme appartenant au système éducatif préscolaire national. La composante éducative du programme vise à développer les compétences et la communication des enfants et à contribuer au renforcement de leur capacité émotionnelle selon leurs besoins à différents âges. Après 3 ans, les enfants peuvent intégrer un programme de jardin d'enfants (CITE 020), supervisé par le Ministère de l'Éducation. (CITE-P : 010)

Brésil - Educação Infantil - crèche (Nurseries). L'Educação Infantil est le niveau d'éducation proposé dans les nurseries (crèches) pour les enfants entre 0 et 3 ans. II s'agit de la première étape de l'éducation de la petite enfance (la seconde étant le préscolaire pour les enfants ayant entre 4 et 5 ans). Conformément à la Loi nationale de l'Éducation № 9394/1996 (le principal instrument juridique d'organisation de l'éducation au Brésil), l'éducation de la petite enfance est la première étape de l'éducation de base et vise le développement physique, psychologique, intellectuel et social des enfants, en complément du rôle de la famille et de la communauté. L'enseignement dans les nurseries est donné par des enseignants qualifiés et suit un programme d'études formel. L'évaluation consiste à contrôler et à enregistrer le développement des enfants sans intention de donner obligatoirement accès à un programme éducatif de niveau plus élevé. (CITE-P : 010)

Ghana - Développement de la petite enfance. Ce programme est destiné aux enfants ayant entre 6 mois et 3 ans. II est supervisé par le Département de l'aide sociale et est assuré par des établissements privés. Après 3 ans, les enfants peuvent intégrer un programme de jardin d'enfants de deux ans (CITE 020), supervisé par le Ministère de l'Éducation. (CITE-P : 010)

Grèce - Vrefonipiakos stathmos (Jardins d'enfants/Éducation de la petite enfance). Ces programmes d'éducation de la petite enfance sont organisés par des jardins d'enfants municipaux ou privés pour de jeunes enfants âgés de 0 à 2 ans. Ils peuvent participer au programme pendant un, deux ou trois ans. Le Vrefonipiakos stathmos satisfait les critères éducatifs pour une classification en tant développement éducatif de la petite enfance dans la CITE 2011. (CITE-P : 010)

\section{Exemples Groupe 2 : \\ Enseignement préprimaire [CITE-P : 020]}

Îles Cook - Éducation de la petite enfance. L'enseignement préprimaire s'adresse aux enfants de 3 à 5 ans. II est assuré dans des Centres d'éducation de la petite enfance, qui dépendent généralement d'écoles primaires. (CITE-P : 020)

Grèce - Nipiagogio (Enseignement préprimaire). Le Nipiagogio est sous la responsabilité du Ministère de l'Éducation. II fait partie de l'enseignement formel en Grèce et a pour objectif de familiariser les enfants avec le processus éducatif et les préparer à l'enseignement primaire (Dimotiko Scholeio, CITE 1). II ne possède pas de composante non éducative. La fréquentation du Nipiagogio est obligatoire depuis 2007 pour les enfants de 5 ans. Les enfants de quatre ans peuvent également fréquenter le Nipiagogio public pour autant qu'il y ait des places disponibles. (CITE-P : 020)

Jordanie - (Jardins d'enfants). II s'agit d'un programme d'enseignement préscolaire dispensé aux enfants ayant au moins 3 ans et 8 mois. Le programme, qui dure jusqu'au commencement de l'enseignement primaire (à 6 ans), ne fait pas partie du système de scolarité obligatoire. (CITE-P : 020) 
Mali - Éducation préscolaire. II s'agit d'un programme d'enseignement préscolaire de quatre ans proposé aux enfants à partir de 3 ans. L'objectif de ce programme non obligatoire est de préparer les enfants à l'enseignement primaire, qui débute à 7 ans. (CITE-P : 020)

République slovaque ${ }^{2}$ - Materská škola (Jardins d'enfants). Les jardins d'enfants sont destinés aux enfants de 3 à 6 ans. Exceptionnellement, si les conditions le permettent (principalement s'il y a suffisamment de places), il est possible d'y accueillir des enfants de 2 ans. Les jardins d'enfants favorisent le développement de la personnalité en termes de capacités et de compétences sociales, intellectuelles, physiques, morales et esthétiques et préparent les enfants à l'entrée dans le Základná škola - 1. stupeň (enseignement de base - 1er cycle, CITE 1). Les jardins d'enfants préparent également à la vie en société en fonction de la personnalité de chaque enfant. (CITE-P : 020)

Turquie - Ana Okullari (Jardins d'enfants). Les jardins d'enfants contribuent à l'éducation des enfants de 3 à 5 ans. Les établissements d'enseignement préprimaire peuvent fonctionner en tant que jardins d'enfants indépendants ou en tant que classes de nurserie dans d'autres établissements d'enseignement. Conformément à la Loi relative aux établissements d'enseignement préprimaire, cet enseignement a pour objectif que les enfants se développent physiquement, mentalement et émotionnellement et acquièrent de bonnes habitudes et de bons comportements ; qu'ils soient préparés pour accéder à l'enseignement primaire ; qu'un environnement commun de développement soit fourni aux enfants provenant d'un milieu défavorisé ; et qu'ils apprennent à parler correctement le Turc. Ces programmes respectent les critères de l'enseignement préprimaire tels que définis dans la CITE 2011. (CITE-P : 020)

Portugal - Jardins de infância (Jardins d'enfants). L'enseignement en jardins d'enfants est destiné aux enfants de 3 à 5 ans et il est sous la responsabilité du Ministère de l'éducation et des Sciences et/ou du Ministère de la Solidarité et de la Sécurité sociale. L'enseignement en jardins d'enfants vise à stimuler le développement cognitif de l'enfant dans un environnement sûr, à vérifier s'il existe des besoins d'enseignement spécial et à encourager les familles à participer au processus éducatif. Cet enseignement est également destiné à préparer les enfants pour leur entrée dans l'enseignement primaire, à encourager leur éducation à la citoyenneté en faveur d'une société plurielle, à stimuler leur curiosité intellectuelle et leurs capacités d'expression et de communication avec le monde. La composante éducative représente 25 heures par semaine et il y a un enseignant qualifié par classe et un enseignant-assistant par deux classes. (CITE-P : 020)

Pologne - Wychowanie przedszkolne (Enseignement préscolaire). Généralement, le temps minimal d'apprentissage dans les classes préscolaires est 5 heures par jour. Ce temps est considéré comme la mise en œuvre d'un programme éducatif (cursus de base) assuré par ce type d'établissement. Le Wychowanie przedszkolne est destiné aux enfants à partir de 3 ans jusqu'à l'âge d'entrée obligatoire dans l'enseignement primaire. Ce programme satisfait les critères de définition et de classification de la CITE 2011 pour l'enseignement préprimaire. II est destiné à répondre aux besoins éducatifs et de développement des enfants, s'adresse habituellement aux enfants ayant au moins 3 ans et dispose de personnel bénéficiant d'une formation adéquate en pédagogie. (CITE-P : 020)

\section{Exemples Groupe 3 : \\ Programmes intégrés [CITE-P : 010, 020]}

Allemagne - Kindergarten, Kindertagesstätte (Jardins d'enfants). L'éducation de la petite enfance comprend tous les établissements gérés par les services d'aide à l'enfance et à la jeunesse non publics ou publics qui répondent aux besoins des enfants jusqu'à leur entrée à l'école. Outre des établissements séparés pour les enfants de moins de 3 ans et pour ceux de 3 à 6 ans, il existe des établissements intégrés en termes d'âge pour les enfants de 0 à 6 ans. Les programmes d'études de la petite enfance des Länder visent des objectifs et des expériences d'apprentissage continu jusqu'à 6 ans. Dans la CITE 2011, les programmes mis en place dans les établissements intégrés en termes d'âge sont affectés aux catégories CITE-P 010 et 020 en fonction de l'âge des enfants inscrits. (CITE-P : 010 et 020)

Slovénie - Pedšolska vzgoja (Enseignement préscolaire). La Slovénie possède un système intégré d'apprentissage et de soins précoces pour les enfants de 1 à 6 ans. Ce système unitaire destiné à tous les enfants dépend du Ministère de l'Éducation, des Sciences, de la Culture et des Sports. L'enseignement préscolaire regroupe les enfants de 1 à 3 ans (1.starostno obdobje, période du premier âge) (CITE-P 010), et de 3 à 6 ans (2.starostno obdobje, période du second âge) (CITE-P 020). Les deux programmes répondent aux critères pour être classés dans le niveau 0 de la CITE. L'enseignement est assuré par des enseignants qualifiés et suit un programme d'études formel. La composante éducative représente plus de 2 heures par jour et l'année scolaire compte plus de 100 jours d'activités éducatives : 97 \% des enfants présents dans les jardins d'enfants suivent des programmes à temps plein et environ $3 \%$ suivent des programmes d'une demi-journée. (Pedšolska vzgoja : (CITE-P : 010 et 020) 
Les pays nordiques se caractérisent par des programmes intégrés d'éducation de la petite enfance. Dans la CITE 2011, ces programmes sont affectés aux catégories CITE-P 010 et 020 en fonction de l'âge des enfants.

Danemark - Aldersintegrerede institutioner (Centre intégré en termes d'âge). Le Aldersintegrerede institutioner est une des composantes du programme de Dagtilbud (nurserie) destiné aux jeunes enfants de la naissance jusqu'à l'âge d'entrée dans l'enseignement primaire obligatoire (folkeskole, niveau 1 de la CITE). Ce programme satisfait les critères pour une classification en tant qu'éducation de la petite enfance dans la CITE 2011. Dans la CITE 2011, le programme Aldersintegrerede institutioner intégré en termes d'âge est subdivisé artificiellement dans les catégories développement éducatif de la petite enfance (CITE-P 010) pour les âges de 0 à 2 ans et enseignement préprimaire (CITE-P 020) pour les âges de 3 à 5 ans. (CITE-P : 010 et 020)

Finlande - Päiväkoti (Jardins d'enfants) Le programme Päiväkoti s'adresse aux enfants de 0 à 5 ans. Après le Päiväkoti, il existe un programme d'un an dénommé 6-v. lasten esiopetus (enseignement préprimaire pour les enfants de 6 ans) organisé dans les jardins d'enfants et les écoles primaires et destiné à préparer les enfants à la Peruskoulu (école primaire, niveau 1 de la CITE). Tous les enfants ont le droit de bénéficier de cette année préprimaire (6-v. lasten esiopetus), mais l'enseignement n'est obligatoire qu'à partir de l'école primaire. Dans la CITE 2011, le programme Päiväkoti intégré en termes d'âge est subdivisé artificiellement dans les catégories destinées aux âges de 0 à 2 ans et de 3 à 5 ans. La catégorie 0-2-v. lapset päiväkodeissa (enfants de 0 à 2 ans dans les jardins d'enfants) est classé en tant que développement éducatif de la petite enfance (CITE-P 010). Ensemble, la catégorie 3-5-v. lapset päiväkodeissa (enfants de 3 à 5 ans dans les jardins d'enfants) et 6-v. lasten esiopetus (préprimaire pour les enfants de 6 ans) regroupent les années préprimaires en Finlande et les deux programmes sont classés en tant que CITE-P 020. (CITE-P : 010 et 020)

Islande - Leikskóli (Jardins d'enfants) Le programme Leikskóli s'adresse aux enfants de 0 à 5 ans. Après le Leikskóli, les enfants à partir de 6 ans peuvent bénéficier de l'enseignement primaire (Grunnskóli I, première phase de la scolarité obligatoire). Dans la CITE 2011, le programme Leikskóli intégré en termes d'âge est subdivisé artificiellement dans la catégorie Leikskóli I destinée aux âges de 0 à 2 ans et la catégorie Leikskóli Il aux âges de 3 à 5 ans. Le Leikskóli I est considéré comme du développement éducatif de la petite enfance (CITE-P 010) et le Leikskóli ll comme de l'enseignement préprimaire (CITE-P 020). (CITE-P : 010 et 020)

Norvège - Barnehage, 0-5 åringer (Jardins d'enfants, de 0 à 5 ans) Le Barnehage est un programme qui s'adresse aux enfants de 0 à 5 ans. II n'est pas obligatoire et l'âge d'entrée typique est 1 an. La certification exigée pour les enseignants est un Bachelorgrad (licence, niveau 6 de la CITE), et le programme respecte un cursus national obligatoire. Après le Barnehage, les enfants débutent l'enseignement primaire (Barnetrinnet, niveau 1 de la CITE), qui est obligatoire. Dans la CITE 2011, le programme Barnehage intégré en termes d'âge est subdivisé artificiellement en Barnehage 1 pour les âges de 0 à 2 ans et Barnehage 2 pour les âges de 3 à 5 ans. Le Barnehage 1 se classe dans le développement éducatif de la petite enfance (CITE-P 010) et le Barnehage 2 dans l'enseignement préprimaire (CITE-P 020). (CITE-P : 010 et 020)

Suède - Förskola (Préscolaire) Le programme Förskola est destiné aux enfants jusqu'à 5 ans. Ce programme éducatif présente également une composante de nurserie. Le Förskola est suivi d'un programme volontaire d'un an nommé Förskoleklass (classe préscolaire) destiné aux enfants de 6 ans. II se déroule dans un environnement de type scolaire. Ensuite, les élèves accèdent à la Grundskolan (école de base, niveau 1 de la CITE) à partir de 7 ans. Dans la CITE 2011, le programme Förskola intégré en termes d'âge se subdivise artificiellement en Förskola för barn under 3 år (pour les enfants de moins de 3 ans) et Förskola för barn 3 år eller äldre (pour les enfants ayant entre 3 et 5 ans). La catégorie Förskola för barn under 3 år est classée en tant que développement éducatif de la petite enfance (CITE-P 010). Ensemble, la Förskola för barn 3 år eller äldre (CITE-P 020) et la Förskoleklass (CITE-P 020) regroupent les années préprimaires en Suède. (CITE-P : 010 et 020)

\section{Exemples Groupe 4 :}

Programmes se déroulant à domicile à inclure dans la CITE 2011 [CITE-P : 010, 020]

Danemark - Kommunal dagpleje (Crèche privée communale). Conformément à la Loi relative aux équipements de nurserie (Dagtilbudsloven), tous les enfants à partir de 26 semaines doivent avoir l'opportunité de bénéficier d'une place en nurserie (Dagtilbud). Cela oblige le conseil local de la municipalité concernée à s'assurer qu'il dispose d'un nombre suffisant de places pour les enfants de la municipalité, ce qui est connu sous le nom de Pasningsgaranti, disponibilité de nurserie garantie. La crèche privée communale (Kommunal dagpleje) est un système où des gardiens d'enfants prennent soin d'enfants dans une maison privée. C'est l'autorité locale qui décide de la répartition des enfants entre les 
différents gardiens d'enfants. Les gardiens d'enfants doivent suivre le même programme d'études et remplir les mêmes obligations en termes de contenu éducatif que les personnes actives dans les nurseries communales (Kommunale daginstitutioner). Un gardien d'enfants peut accueillir cinq enfants au plus. Si deux personnes ou davantage accueillent des enfants ensemble, l'autorité locale peut leur permettre de recevoir jusqu'à dix enfants. Les programmes d'accueil communaux satisfont les principaux critères de la CITE 2011 pour la classification au niveau 0 de la CITE : propriétés éducatives, contexte institutionnel, groupes d'âge cibles, intensité et durée. Dans le cadre de la soumission des données, les données relatives aux enfants de moins de 3 ans sont rapportées comme CITE 010 ; les données relatives aux enfants de 3 ans jusqu'à l'âge d'entrée dans la CITE 1 sont rapportées comme CITE-P 020. (CITE-P : 010 et 020)

Allemagne - Kindertagespflege (Services d'accueil d'enfants). Pour les enfants de moins de 3 ans, l'accueil dans des établissements ou à domicile est considéré légalement comme des alternatives équivalentes ; les deux possèdent un contenu éducatif explicite ; c'est la raison pour laquelle la fréquentation d'une crèche à domicile (garde d'enfants) sera rapportée dans la CITE-P : 010. Concernant les enfants ayant entre 3 ans et l'âge obligatoire de scolarisation, I'utilisation des services de garde d'enfants est très rare et presque toujours associée à un accueil dans un établissement; pour la soumission des données dans la CITE-P : 020, l'aspect garde d'enfants n'a par conséquent guère d'importance. (Garde d'enfants de moins de 3 ans : (CITE-P : 010)

Exemples Groupe 5 :

Programmes se déroulant à domicile à exclure du niveau 0 de la CITE 2011 [Pas de classification]

Exemple général : Un exemple serait un citoyen privé qui offrirait volontairement des opportunités d'apprentissage à de jeunes enfants qui rempliraient les critères de niveau de la CITE concernant l'éducation volontaire, l'intensité/la durée et les exigences liées aux certifications du personnel, mais qui ne seraient pas institutionnalisées ni règlementées par une quelconque autorité compétente en matière d'éducation.

Danemark - Private Pasningsordninger (Garde d'enfants privée). Des gardes d'enfants privés peuvent également accueillir de jour des enfants ayant au moins 26 semaines sur la base d'un contrat de fonctionnement avec l'autorité locale. Ce n'est pas l'autorité locale qui décide de la répartition des enfants entre les gardes d'enfants privés. Bien que l'autorité locale verse un montant convenu au garde d'enfants pour chaque enfant et supervise le système, il n'y a pas d'obligation de cursus ou de contenu éducatif. Étant donné qu'il n'y a pas de contenu éducatif élaboré, ce type de garde ne serait pas classé au niveau 0 de la CITE.

\section{Notes}

1. Les numéros de paragraphe constituent des références à la classification CITE 2011. Pour plus de détails, veuillez consulter le Guide du lecteur.

2. L'Institut de statistique de I'UNESCO (ISU) et I'Union européenne (UE) utilisent « Slovaquie " comme dénomination officielle de la "République slovaque ". 



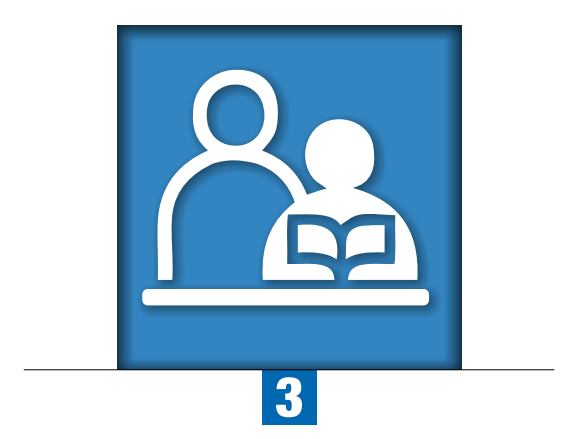

\section{Chapitre 3}

\section{Niveau 1 de la CITE 2011:}

\section{Enseignement primaire}

L'enseignement primaire débute généralement à 5,6 ou 7 ans et a une durée typique de six ans. Les programmes du niveau 1 de la CITE sont généralement conçus pour donner aux élèves des aptitudes fondamentales en lecture, écriture et mathématiques et établir une base solide pour la compréhension d'autres matières telles que l'histoire, la géographie, les sciences naturelles, les sciences sociales, l'art et la musique. Le commencement des activités de lecture n'est pas un critère suffisant à lui seul pour la classification d'un programme éducatif dans le niveau 1 de la CITE.

Les programmes classés au niveau 1 de la CITE peuvent être désignés de plusieurs manières, par exemple : enseignement primaire, enseignement élémentaire ou éducation de base (cycle 1 ou premières années d'études si le système éducatif est caractérisé par un programme qui couvre les niveaux 1 et 2 de la CITE). En vue de la comparabilité internationale, le terme " enseignement primaire » est utilisé pour qualifier le niveau 1 de la CITE à l'échelle internationale. 


\section{DESCRIPTION}

\section{Définition}

$(\S 120)^{1}$ Les programmes du niveau 1 de la CITE, ou « enseignement primaire ", sont généralement conçus pour donner aux élèves des aptitudes fondamentales en lecture, écriture et mathématiques (c'est-à-dire l'alphabétisme et le calcul) et établir une base solide pour l'apprentissage et la compréhension des connaissances de base, le développement personnel et social et la préparation au premier cycle de l'enseignement secondaire. II se concentre sur un apprentissage avec un niveau de complexité de base et peu ou pas de spécialisation.

(\$121) Les activités éducatives du niveau 1 de la CITE (particulièrement dans les premières années d'études) s'articulent souvent autour d'unités, de projets ou de grands domaines d'apprentissage, principalement avec une approche intégrée et non l'enseignement de matières spécifiques.

\section{Critères de classification (\$124)}

\section{Critères principaux}

a. Enseignement systématique de connaissances, d'aptitudes et compétences élémentaires

(\$125) La limite entre les niveaux 0 et 1 de la CITE coïncide avec le point de transition où l'enseignement et l'apprentissage systématiques de la lecture, de l'écriture et des mathématiques débutent dans un système éducatif.

b. Âge normal d'entrée et durée

(§122) L'âge est normalement le seul critère d'admission dans ce niveau. L'âge habituel ou légal d'admission n'est généralement ni inférieur à 5 ans, ni supérieur à 7 ans. Ce niveau dure généralement 6 ans, même si sa durée peut varier de 4 à 7 ans. L'enseignement primaire se termine habituellement entre 10 et 12 ans.

c. Enseignement généralement organisé par un enseignant principal pour la classe

(§126) Le plus souvent, un enseignant principal est responsable d'un groupe d'enfants et assure le processus d'apprentissage. Toutefois, une classe peut avoir d'autres enseignants, surtout pour certaines matières spécialisées. Dans la plupart des cas, les enseignants du niveau 1 de la CITE ont suivi une formation afin d'acquérir une approche pédagogique des principales matières.

\section{Critères subsidiaires}

a. Fait partie de la scolarité obligatoire

(§127) Le début de l'enseignement primaire coïncide souvent avec le début de la scolarité obligatoire, excepté pour les systèmes éducatifs où une partie ou la totalité de l'enseignement préprimaire est déjà obligatoire. Dès lors, là où la scolarité obligatoire est imposée légalement, elle commence au niveau 1 de la CITE ou avant.

\section{Dimensions complémentaires}

(§131) Aucune.

Tous les programmes éducatifs du niveau 1 de la CITE prennent le code CITE-P 100. II n'y a pas de catégorie ou de sous-catégorie différenciée par un deuxième chiffre. (\$135)

\section{CORRESPONDANCES ENTRE LA CITE 2011 ET LA CITE 1997}

(§276) Le niveau 1, enseignement primaire, de la CITE 2011 correspond au niveau 1 de la CITE 1997. 


\section{DIRECTIVES POUR LA CLASSIFICATION DES PROGRAMMES ET DE LEURS CERTIFICATIONS RECONNUES CORRESPONDANTES AU NIVEAU 1 DE LA CITE}

L'enseignement primaire débute généralement à 5, 6 ou 7 ans et a une durée typique de six ans. Les programmes du niveau 1 de la CITE sont généralement conçus pour donner aux élèves des aptitudes fondamentales en lecture, en écriture et en mathématiques et établir une base solide pour la compréhension d'autres matières telles que l'histoire, la géographie, les sciences naturelles, les sciences sociales, l'art et la musique. Le commencement des activités de lecture n'est pas un critère suffisant à lui seul pour la classification d'un programme éducatif dans le niveau 1 de la CITE. Généralement, il y a un enseignant principal par classe à ce niveau, bien qu'il puisse y avoir d'autres enseignants pour certaines matières spécialisées. Les enseignants de primaire sont généralement formés aux méthodes pédagogiques permettant d'enseigner de nombreuses matières différentes. Voir Exemples Groupe 1 pour des exemples de programmes typiques de l'enseignement primaire.

L'âge est normalement le seul critère d'admission dans le niveau 1 de la CITE. Les programmes du niveau primaire ne nécessitent généralement pas d'enseignement formel préalable, bien qu'il soit de plus en plus commun que les enfants aient participé à un programme préprimaire avant l'entrée dans l'enseignement primaire. L'âge d'entrée dans l'enseignement primaire coïncide souvent avec le début de la scolarité obligatoire, bien que dans certains pays la scolarité obligatoire débute au niveau préprimaire.

Si la durée typique de l'enseignement primaire est de six ans, elle peut varier de quatre à sept ans selon les pays. De plus, la durée de l'enseignement primaire peut varier à l'intérieur même d'un pays, notamment les pays fédéraux avec des systèmes éducatifs décentralisés par région (voir Exemples Groupe 2).

Le niveau 1 de la CITE comprend des programmes à l'intention des enfants qui ont des besoins spéciaux (handicaps, difficultés ou désavantages) si le contenu de l'enseignement est largement similaire à celui d'autres programmes du niveau 1 de la CITE (c'est-à-dire avec un enseignement et un apprentissage systématiques des compétences fondamentales en lecture, en écriture et en mathématiques), même si les élèves n'ont pas l'âge typique du niveau 1 de la CITE (§132) (Exemples Groupe 3).

Certains systèmes éducatifs intègrent également des programmes de seconde chance ou de réintégration de niveau primaire. Ces programmes éducatifs s'adressent en général à des individus qui :

i) ont quitté l'école avant d'avoir achevé l'enseignement primaire ; ils leur permettent de réintégrer le système éducatif et d'achever avec succès cet enseignement ; ou

ii) ont achevé l'enseignement primaire mais souhaitent accéder à un programme éducatif ou à une profession pour lesquels ils ne sont pas encore certifiés. Les participants sont habituellement plus âgés que le groupe d'âge ciblé par le niveau 1 de la CITE (mais ce ne sont pas nécessairement des adultes). (§133)

Les programmes d'alphabétisation ou d'enseignement de compétences de base, dans ou hors du système scolaire, dont le contenu est similaire à celui des programmes déjà classés dans l'enseignement primaire mais qui sont destinés à des personnes considérées comme trop âgées pour entrer à l'école sont également inclus dans ce niveau car ils n'exigent pas d'enseignement formel préalable. Ces programmes d'alphabétisation peuvent être formels et non formels (§134). Voir Exemples Groupe 4 pour des exemples de programmes pour jeunes ou pour adultes n'ayant pas l'âge typique pour l'enseignement primaire.

Un critère d'approximation supplémentaire de classification au niveau 1 de la CITE pourrait être l'entrée dans les programmes ou établissements scolaires désignés comme primaires à l'échelle nationale. Toutefois, dans les pays où l'enseignement primaire débute tôt (par ex. 4 ou 4,5 ans), les enfants scolarisés dans ces programmes ne doivent être classés au niveau 1 de la CITE que si la durée de la journée d'école, les qualifications du personnel et le niveau du contenu du programme sont similaires aux années d'études dans lesquelles les enfants de 6 ans sont scolarisés. Dans le cas contraire, toute année d'études précédente correspondant plutôt au niveau 0 de la CITE doit être classée dans l'enseignement préprimaire plutôt que dans le primaire.

Bien que le début de la scolarité obligatoire constitue un critère subsidiaire de distinction entre les niveaux 0 et 1 de la CITE, ce critère n'est pas utile dans les pays où le début de la scolarité obligatoire n'est pas nécessairement lié au début d'études systématiques. Dans les pays où l'âge de fréquentation obligatoire (ou du moins l'âge auquel les élèves commencent normalement leur éducation) est postérieur au début des études systématiques pour certains élèves dans 
les matières mentionnées ci-dessus, la première année de fréquentation obligatoire peut être utilisée pour opérer la distinction entre le niveau 0 et le niveau 1 de la CITE. Ce dernier critère peut être utile lorsque le début du niveau 1 de la CITE reflète le point auquel les études systématiques dans les matières ci-dessus commencent pour tous les élèves, et non pour quelques-uns.

\section{Programmes couvrant l'enseignement primaire et un autre niveau de la CITE}

II faut être particulièrement attentif aux très longs programmes (c'est-à-dire huit ans ou plus) couvrant l'enseignement primaire et les niveaux précédents ou postérieurs. Dans ces cas, seuls les années d'études, phases ou cycles correspondant aux critères des niveaux respectifs (niveau 0, 1 ou 2 de la CITE) doivent être classés dans ces niveaux. (§128)

Pour les cas où l'utilisation des critères de classification ne permet pas d'établir une distinction claire entre les niveaux 0 et 1 de la CITE, veuillez consulter le chapitre sur le niveau 0 de la CITE du présent Guide opérationnel.

Dans la plupart des pays, le niveau 1 de la CITE correspond à l'enseignement désigné comme primaire. Dans les pays où l' "éducation de base " couvre la totalité de la durée de scolarité obligatoire (c'est-à-dire qu'il n'y a pas de distinction entre l'enseignement primaire et le premier cycle de l'enseignement secondaire, et éventuellement aussi une partie de l'éducation de la petite enfance), seules les années d'études correspondant aux critères du niveau 1 de la CITE doivent être classées dans l'enseignement primaire.

Si l'utilisation des critères de classification ne permet pas d'établir une distinction claire entre les niveaux 1 et 2 de la CITE, il est recommandé de procéder comme suit : (\$130)

i) pour les programmes couvrant les niveaux 1 et 2 de la CITE et organisés par cycle, la fin de cycle la plus proche de six ans après le début du niveau 1 de la CITE doit être utilisée comme point de transition entre les niveaux 1 et 2 de la CITE ; et

ii) pour les programmes couvrant les niveaux 1 et 2 de la CITE qui ne sont pas répartis en cycles, seules les six premières années d'études doivent être classées dans le niveau 1 de la CITE, alors que les années d'études suivantes sont classées dans le niveau 2 de la CITE.

Voir Exemples Groupe 5 pour les programmes couvrant les niveaux 1 et 2 de la CITE.

\section{NIVEAU D'ÉDUCATION ATTEINT AU NIVEAU 1 DE LA CITE}

Dans le cadre de la codification du niveau d'éducation atteint, une attention particulière doit être accordée à la classification des individus qui ont suivi un enseignement primaire, mais qui ne l'ont pas achevé (\$136). Ces individus ont le code de niveau d'éducation atteint CITE-A 030 "Scolarisation primaire limitée (sans achèvement de niveau) " (Exemples Groupe 6).

Les élèves ayant achevé le niveau 1 de la CITE ont le code de niveau d'éducation atteint CITE-A 100.

En ce qui concerne le niveau d'éducation atteint, les certifications reconnues résultant des programmes du niveau 2 de la CITE considérés comme insuffisants pour être pris en compte comme un achèvement partiel ou complet du niveau 2 de la CITE (soit CITE-P 241 ou 251) sont classées dans le niveau 1 de la CITE (CITE-A 100). Ces programmes ont généralement une durée totale inférieure à deux ans au niveau 2 de la CITE ou une durée cumulée inférieure à huit ans depuis le début du niveau 1 de la CITE. (§137)

\section{EXEMPLES DE PROGRAMMES ET DE CERTIFICATIONS CLASSÉS AU NIVEAU 1 DE LA CITE}

(§123) Les programmes classés au niveau 1 de la CITE peuvent être désignés de plusieurs manières, par exemple : enseignement primaire, enseignement élémentaire ou éducation de base (cycle 1 ou premières années d'études si le système éducatif est caractérisé par un programme qui couvre les niveaux 1 et 2 de la CITE). En vue de la comparabilité internationale, le terme " enseignement primaire " est utilisé pour qualifier le niveau 1 de la CITE à l'échelle internationale. 
Exemples Groupe 1 :

Programmes typiques de l'enseignement primaire [CITE-P : 100 ; CITE A : 100]

Autriche - Volksschule, 1-4 schulstufe (école primaire, Années d'études 1 à 4). L'enseignement primaire est obligatoire pour tous les enfants à partir de 6 ans. II dure quatre ans et est donné dans des écoles primaires (Volksschule). L'enseignement primaire s'organise normalement en deux niveaux : les deux premières années (niveau l) et les années 3 et 4 (niveau II). À l'achèvement de l'année 4, les élèves reçoivent une certification. L'enseignement primaire pose les fondements d'une expérience d'apprentissage réussie dans l'enseignement secondaire. En fonction de la formation de chaque élève, l'école primaire doit : renforcer et développer des compétences sociales, améliorer les compétences linguistiques, développer et transmettre des connaissances, des compétences, des idées et des attitudes élémentaires menant à l'acquisition des compétences fondamentales (lire, écrire, compter). (CITE-P/CITE-A : 100)

Belgique (Communauté flamande) - Gewoon lager onderwijs (enseignement primaire général). L'enseignement primaire général accueille des enfants de 6 à 12 ans et compte généralement six années d'études cumulées (même si la durée peut varier de quatre à huit ans). L'apprentissage systématique de la lecture, de l'écriture et des mathématiques débute dans la première année. Chaque groupe d'élèves a son propre enseignant chargé de la plupart (ou même la totalité) des matières. Un enseignant différent peut être désigné pour l'instruction religieuse, la musique et l'éducation physique. L'enseignement primaire général débute en septembre de l'année où l'enfant atteint 6 ans. Pour un enfant progressant normalement, le début de la scolarité obligatoire coïncide avec le début de l'enseignement primaire. Lors de l'achèvement complet de l'enseignement primaire, les élèves reçoivent la certification de l'enseignement primaire. (CITE-P/CITE-A : 100)

France - Enseignement primaire. L'enseignement primaire dure cinq ans et est obligatoire pour tous les enfants à partir de 6 ans. Le programme commence par un cycle d'apprentissage fondamental comprenant une année de cours préparatoire $(\mathrm{CP})$ et la première année de cours élémentaire (CE1). Les trois dernières années d'enseignement primaire comprennent la seconde année de cours élémentaire (CE2) et deux années de cours moyen (CM1 et CM2). Chaque fin de cycle se clôture par une évaluation nationale permettant de mesurer le niveau d'apprentissage des élèves. Néanmoins, aucune certification n'est attribuée lors de l'achèvement de l'enseignement primaire. Après avoir achevé l'enseignement primaire, les élèves accèdent à l'enseignement secondaire. (CITE-P/CITE-A : 100)

Grèce - Dimotiko Scholeio (Écoles élémentaires). L'enseignement primaire est obligatoire et dure 6 ans (Années d'études 1 à 6) pour tous les enfants à partir de 6 ans. II permet aux élèves de développer des stratégies en vue d'assimiler des connaissances ; de renforcer leur santé physique et mentale et de cultiver leur habileté motrice ; d'apprendre les fondamentaux et progressivement d'acquérir la capacité de parvenir à des pensées abstraites à partir de données concrètes ; d'acquérir la capacité de s'exprimer par l'oral et par l'écrit ; de se familiariser avec des valeurs éthiques, religieuses, nationales et humanistes ; et de cultiver leurs talents artistiques. À l'achèvement de l'année 6, les élèves reçoivent une certification de fin d'études primaires (Titulos spoudon) qui leur donne accès au premier cycle du secondaire (gymnasium). (CITE-P/CITE-A : 100)

Inde - Enseignement primaire. L'Inde se caractérise par des systèmes éducatifs variés. L'enseignement primaire le plus commun dans les états et les territoires dure cinq ans (Classes I-V) et commence à cinq ans. Ce programme gratuit et obligatoire débouche sur une certification de fin d'études primaires qui donne accès au premier cycle de l'enseignement secondaire. (CITE-P/CITE-A : 100)

Italie - Scuola primaria (École primaire). L'enseignement primaire correspond aux cinq premières années du premier cycle d'enseignement. La fréquentation est obligatoire pour tous les enfants de 6 à 10 ans. L'enseignement primaire présente trois cycles : une première année (Année d'études 1), qui vise à faciliter la transition à partir de l'enseignement préprimaire (scuola dell'infanzia), suivie de deux cycles de deux années (Années d'études 2 et 3, et Années d'études 4 et 5). Au terme de l'enseignement primaire, les élèves reçoivent un document d'évaluation personnelle spécifiant si l'élève peut être admis dans la première année d'études du premier cycle de l'enseignement secondaire (scuola secondaria di primo grado), ainsi qu'une certification des compétences acquises par l'élève à l'achèvement de l'école primaire (Certificati e/o Attestati). (CITE-P/CITE-A : 100)

Japon - Shogakko (École élémentaire). L'enseignement élémentaire est la première étape de la scolarité obligatoire. Tous les enfants fréquentent l'école élémentaire pendant six ans à partir de l'âge de six ans. Les objectifs de l'enseignement élémentaire sont : compréhension et utilisation du japonais et des mathématiques, compréhension de base des 
compétences nécessaires dans la vie quotidienne, observation scientifique de la nature, initiation à l'histoire, à l'art et à la musique. Après l'achèvement complet de l'enseignement élémentaire, les élèves passent dans le premier cycle de l'école secondaire (Chugakko). (CITE-P/CITE-A : 100)

Corée ${ }^{2}$ - Chodeung-hakgyo (École élémentaire). L'enseignement primaire est donné dans des écoles élémentaires et s'adresse aux enfants de 6 à 11 ans (Années d'études 1 à 6). Généralement, l'enseignement élémentaire a pour objectif de favoriser le développement d'individus sains, autonomes, créatifs et dotés de sens moral. Pour cela, on renforce leur capacité à exprimer leurs sentiments et leurs idées, à résoudre des problèmes, à apprécier la culture et la diversité, et on favorise l'acquisition des compétences de base pour la vie. Quand ils achèvent avec succès l'enseignement élémentaire, les élèves reçoivent une certification (Joleopjang) et peuvent accéder à l'école moyenne (Jung-hakgyo) (CITE 2). (CITE-P/ CITE-A : 100)

Liban - (Enseignement primaire). L'enseignement primaire couvre les deux premiers cycles de l'enseignement de base obligatoire, qui dure neuf ans au total. Chacun de ces deux cycles compte trois années d'études. Le programme d'enseignement primaire vise normalement les enfants à partir de 6 ans ; ils apprennent les compétences fondamentales en matière de lecture et de mathématiques. À ce niveau, on les initie également à une langue étrangère (soit l'anglais, soit le français). Après un achèvement complet, les élèves peuvent accéder au troisième cycle de l'enseignement de base (premier cycle de l'enseignement secondaire). (CITE-P/CITE-A : 100)

Îles Salomon - Enseignement conventionnel. L'enseignement conventionnel débute à six ans et dure six ans, de l'année 1 à l'année 6. Au terme de l'année 6, les élèves sont soumis à un examen formel marquant la fin de l'école primaire. Le programme d'études comprend l'écriture, la lecture, les mathématiques, les études communautaires, les sciences, l'agriculture, l'art, la musique, l'éducation physique et l'éducation chrétienne. (CITE-P/CITE-A : 100)

Ukraine - (Enseignement primaire). II s'agit d'un programme éducatif obligatoire de quatre ans destiné aux enfants à partir de six ans et il se déroule dans des écoles élémentaires. Chaque groupe d'élèves a son propre enseignant qui enseigne la plupart des matières. Un enseignant différent peut être désigné pour la musique et l'éducation physique. Lors de l'achèvement complet du programme, les élèves reçoivent la certification de l'enseignement élémentaire. (CITE-P/ CITE-A : 100)

Exemples Groupe 2 :

Pays avec une variabilité nationale dans la longueur des programmes de primaire [CITE-P : 100 ; CITE A : 100]

Canada et États-Unis - Écoles élémentaires/primaires. L'enseignement primaire et secondaire forme une continuité, la durée de l'école élémentaire ou primaire se fondant principalement sur des caractéristiques institutionnelles qui peuvent varier d'une province/d'un état ou d'une localité à l'autre (allant de trois à huit années d'études). Dans ces pays, la continuité élémentaire-secondaire sera subdivisée au terme de l'année d'études 6 pour la soumission des données dans le niveau 1 de la CITE, de sorte que les années d'études de chaque niveau facilitent la comparabilité internationale. Cette méthode de soumission des données relatives aux programmes assurera également que, dans un contexte national, des programmes comparables soient affectés à chaque niveau puisque le contenu pour une année d'études est largement similaire dans les provinces et états. (CITE-P/CITE-A : 100)

Allemagne - Primarbereich (Enseignement primaire). L'enseignement primaire est la première étape de la scolarité obligatoire et couvre les Années d'études 1 à 4 dans la plus grande partie de Allemagne, ou les Années d'études 1 à 6 à Berlin et au Brandebourg. Tous les enfants fréquentent l'école primaire (Grundschulen) dans le cadre de leur scolarité obligatoire entre 6 et 10 ans (à Berlin et au Brandebourg entre 6 et 12 ans). L'école primaire a pour objectif de mener ses élèves de formes d'apprentissage plutôt axées sur le jeu au niveau préscolaire vers des formes d'apprentissage scolaire plus systématiques, apportant ainsi aux élèves les bases nécessaires pour le niveau éducatif suivant (Sekundarbereich I: premier cycle de l'enseignement secondaire) et l'apprentissage tout au long de la vie. Les cours de l'école primaire mettent l'accent sur la lecture, l'écriture, l'arithmétique, les sciences et une langue étrangère. L'enseignement se déroule au travers de cours focalisés sur une matière spécifique et de classes multidisciplinaires. Pour assurer une approche pédagogique cohérente, on applique le principe d'un enseignant par classe couvrant toutes les matières ou presque. À partir de la $3^{e}$ année d'études, les enfants sont de plus en plus confrontés à des enseignants spécialisés, ce qui facilite leur transition vers l'école secondaire, où les enseignants spécialisés sont la règle. Aux fins de la soumission de données internationale, on considère que la durée de la CITE 1 est de quatre ans (Années d'études 1 à 4) en Allemagne. (CITE-P/ CITE-A : 100) 
Suisse - Primarschule, école primaire, scuola elementare. L'âge d'entrée dans l'enseignement primaire est soit 6 ans (4 cantons), 6,5 ans (2 cantons) ou 7 ans (17 cantons). Un canton permet aux communes (autorités locales) de décider si l'école commence à 6 ou 7 ans. Comme la durée combinée du niveau primaire et du premier cycle du secondaire combinés est partout de neuf ans, les différences dans les âges d'entrée se traduisent par des différences dans les âges d'entrée pendant tout le parcours scolaire des élèves. L'enseignement primaire dure entre quatre et six ans (selon le canton). Des réformes en cours réduiront la part des élèves dans des programmes de quatre ans. À des fins de comparabilité, les six premières années du primaire/premier cycle de l'enseignement secondaire seront affectées au Niveau 1 de la CITE. (CITE-P/CITE-A : 100)

Royaume-Uni - École primaire. L'âge d'entrée à l'école primaire est 5 ans en Angleterre, en Écosse et au Pays de Galles, et 4 ans en Irlande du Nord alors que sa durée varie entre six ans (Angleterre et Pays de Galles) et sept ans (Irlande du Nord et Écosse). (CITE-P/CITE-A : 100)

\section{Exemples Groupe 3 :}

Programmes de l'enseignement primaire destinés aux enfants avec des besoins éducatifs spéciaux [CITE-P : 100 ; CITE-A : 100]

Belgique (Communauté flamande) - Buitengewoon lager onderwijs (Enseignement primaire spécial). L'enseignement primaire spécial s'adresse aux enfants ayant besoin d'une aide spéciale, temporairement ou de manière permanente. Cela peut être dû à un handicap physique ou mental, des problèmes comportementaux ou émotionnels graves, ou de sérieuses difficultés d'apprentissage. L'entrée dans l'enseignement primaire spécial se fonde sur l'âge de l'élève : 6 ans avant le 1er janvier de l'année scolaire. Une entrée est également possible à 5 ans, ce que les parents peuvent décider sur la base des conseils du comité de classe et le centre d'orientation des élèves. Dans l'enseignement spécial, un projet scolaire spécial est établi (avec le type d'enseignement pour l'élève, sur la base du type de handicap). Au terme du programme de sept ans, les élèves reçoivent une certification de fin d'enseignement primaire (Getuigschrift Basisonderwijs) et peuvent poursuivre leurs études dans la Gewoon secundair onderwijs - 1ste graad (première année d'études de l'enseignement secondaire, CITE 2). (CITE-P/CITE-A : 100)

République slovaque ${ }^{3}$ - Špeciálna základná škola - 1 stupeň (École de base spéciale - premier cycle). II existe divers programmes éducatifs pour les élèves ayant des besoins éducatifs spéciaux proposés parallèlement à l'enseignement primaire normal (Základná škola - 1, stupeň / école de base - premier cycle, CITE 1). L'école de base spéciale est destinée aux enfants souffrant de handicaps physiques. II existe aussi des variantes de programmes éducatifs de base pour les enfants avec des difficultés d'apprentissage et des handicaps mentaux. À 6 ans, l'âge de début de dix ans de scolarité obligatoire, les élèves peuvent démarrer le programme spécial à plein temps de quatre ans. Après un achèvement complet, les élèves reçoivent un Vysvedčenie (certificat) et peuvent accéder au second cycle de l'école de base (CITE 2). (CITE-P/CITE-A : 100)

\section{Exemples Groupe 4 : \\ Programmes de l'enseignement primaire pour les personnes n'ayant pas l'âge typique de la scolarité primaire [CITE-P : 100 ; CITE-A : 100]}

Belgique (Communauté flamande) - Basiseducatie (Éducation de base pour adultes). En Communauté flamande de Belgique, l'éducation de base pour adultes vise une amélioration du niveau général en lecture et en calcul, du néerlandais comme seconde langue, les bases du français et de l'anglais, l'intégration sociale et les compétences informatiques de base. L'éducation de base pour adultes ne s'organise que dans des centres d'éducation de base pour adultes. Ces cours portant sur des compétences de base sont affectés au niveau de l'enseignement primaire et au premier cycle de l'enseignement secondaire (CITE 1 et CITE 2) selon leurs niveaux de contenu. (CITE-P/CITE-A : 100)

Égypte - (Enseignement communautaire). Ce programme, organisé par le Ministère de l'éducation et soutenu par les communautés locales ainsi que des ONG locales et internationales, cible les enfants non scolarisés ayant entre 6 et 14 ans. Il offre une seconde chance aux enfants qui n'ont jamais été scolarisés dans l'enseignement formel et à ceux qui ont quitté l'école avant d'achever l'enseignement primaire. Dans les écoles communautaires, les classes sont généralement limitées à 30 élèves dans un groupe multi-âge supervisé par deux facilitateurs. Les élèves sont répartis en fonction de leurs capacités et de la vitesse à laquelle ils apprennent, mais ils sont tous rassemblés dans une seule classe. Bien que le programme soit conçu selon l'enseignement primaire initial - avec une durée de six ans - les élèves plus rapides peuvent 
achever le programme en trois ans seulement. Après un achèvement complet, les élèves reçoivent une certification de fin d'école primaire et peuvent accéder à l'école préparatoire. (CITE-P/CITE-A : 100)

Hongrie - Általános iskola 1-4, évfolyam, felnõttoktatás (École générale de niveau primaire, Années d'études 1 à 4 , enseignement à temps partiel, cours d'alphabétisation pour adultes). II s'agit d'un programme qui s'adresse aux élèves à partir de 16 ans et apporte des compétences en lecture et un enseignement similaire à celui fourni dans le programme de niveau primaire général à plein temps de quatre ans (CITE 1). Lors de l'achèvement complet du programme, les élèves reçoivent un certificat (Bizonyitvány) et peuvent accéder au second cycle de l'école secondaire générale (CITE 2). (CITE-P/CITE-A : 100)

Koweit - (Enseignement primaire pour adultes). Le programme d'enseignement primaire pour adultes est destiné aux jeunes de 15 ans et plus qui, soit n'ont jamais été scolarisés dans l'enseignement formel, soit l'ont quitté quand ils étaient plus jeunes. Le programme vise à offrir une seconde chance et à réintégrer les jeunes dans l'enseignement initial. Contrairement au programme primaire initial, qui dure cinq ans, le programme pour adultes ne dure que trois ans. À l'achèvement de ce programme formel, les élèves se voient décerner une certification de fin de primaire et peuvent poursuivre leurs études dans le cycle intermédiaire pour adultes (CITE 2). (CITE-P/CITE-A : 100)

Soudan du Sud - Programme d'Apprentissage accéléré (PAA) au niveau primaire. Ce programme est proposé dans le cadre du Système d'enseignement alternatif. II est destiné à aider ceux qui ont abandonné tôt l'école, enfants ou adultes, à accélérer leur apprentissage, soit pour (ré)intégrer le système éducatif initial, soit pour acquérir quelques compétences en vue de mener une vie productive. Le PAA ramène les huit années normales d'enseignement primaire à quatre ans. La majorité des participants ont plus de 10 ans, alors que l'âge officiel d'entrée dans l'enseignement primaire est 6 ans. À l'achèvement du programme, les participants obtiennent une certification de l'enseignement primaire et peuvent accéder à l'enseignement secondaire. (CITE-P/CITE-A : 100)

Espagne - Enseñanzas Iniciales de Educación Básica para personas en edad adulta (Programme d'éducation pour adultes au niveau primaire). II s'agit de programmes de seconde chance pour les personnes ayant dépassé les âges de la scolarité obligatoire. Ils visent spécifiquement l'alphabétisation et la consolidation des connaissances de base des participants. II n'y a pas de durée théorique définie, ni de restrictions liées à une durée maximale ou minimale. L'enseignement fourni est similaire à celui de l'enseignement primaire à plein temps de six ans (Educación primaria, CITE 1) ; dès lors, la durée théorique est considérée comme équivalente à l'enseignement primaire ordinaire. Lors de l'achèvement complet du programme, les élèves peuvent accéder à l'Educación secundaria (premier cycle de l'enseignement secondaire). (CITE-P/CITE-A : 100)

Suède - Svenska för vuxna invandrare (Suédois pour les immigrants adultes). Ce programme d'un an enseigne le suédois et des informations générales sur la vie en Suède à des immigrants adultes de 16 ans ou plus. II est classé au niveau enseignement primaire du pays. Après avoir achevé le programme, les élèves ont le même niveau d'éducation atteint que celui qu'ils avaient avant leur entrée. (CITE-P : 100 ; CITE-A : identique à celui avant d'entrer)

\section{Exemples Groupe 5 : \\ Programmes couvrant les niveaux 1 et 2 de la CITE}

République tchèque - Základni škola (École de base). Les écoles de base fournissent neuf ans d'éducation de base obligatoire, qui débute généralement quand l'enfant a 6 ans. Elle se répartit en deux cycles. Le premier cycle de l'école de base couvre les Années d'études 1 à 5 (enseignement primaire, CITE 1) et le second les Années d'études 6 à 9 (premier cycle de secondaire, CITE 2). Au cours des Années d'études 1 à 5 (le premier cycle de l'école de base), l'enseignement et le processus d'apprentissage ont pour objectif de faciliter la transition de l'enseignement préscolaire (CITE 0) et de la prise en charge par la famille vers un enseignement obligatoire, régulier et systématique. Étant donné son organisation fondée sur des activités et sa nature pratique, le processus éducatif motive les élèves à poursuivre leur apprentissage, les guide vers un apprentissage actif et enseigne les compétences de résolution de problèmes. Le premier cycle du programme d'éducation de base est destiné à enseigner des connaissances fondamentales en matière d'éducation générale, polytechnique, physique et esthétique. (Années d'études 1 à 5 : CITE-P/CITE-A : 100 ; Années d'études 6 à 9 : CITE-P/CITE-A : 244)

Danemark - Grundskole (École générale). La Grundskole compte dix années d'études. II est obligatoire de suivre dix ans d'enseignement au niveau grundskole. Toutefois, il n'est pas obligatoire de suivre cet enseignement dans des établissements scolaires formels. La grundskole consiste en une classe préscolaire (bornehaveklasse) pour les enfants 
de 6 ans suivie de neuf années d'éducation de base. Dans la CITE 2011, la classe préscolaire et les Années d'études 1 à 6 d'éducation de base (grundskole, 1-6 klasse) sont classées dans l'enseignement primaire (Niveau 1 de la CITE) et les Années d'études 7 à 9 d'éducation de base (grundskole, 7-9 klasse) sont considérées comme appartenant au premier cycle de l'enseignement secondaire (Niveau 2 de la CITE). Les dix années d'école générale sont organisées sur la base de trois groupes de matières (humanités, matières pratiques/créatives et sciences), complétés par des matières au choix dans les trois dernières années (CITE 2), des matières supplémentaires parmi lesquelles les élèves peuvent choisir. (Préscolaire à l'Année d'études 6 : CITE-P/CITE-A : 100 ; Années d'études 7 à 9 : CITE-P/CITE-A : 244)

Estonie - Põhidaridus, põhikooli (Éducation de base). L'éducation de base est obligatoire pour tous les enfants à partir de 7 ans et elle dure neuf ans (Années d'études 1 à 9). L'éducation de base s'organise sous la forme de trois cycles de trois ans chacun : Années d'études 1 à 3, Années d'études 4 à 6 et Années d'études 7 à 9 . Les élèves des Années d'études 7 à 9 peuvent également bénéficier d'une formation préprofessionnelle dans des écoles de base. Dans la CITE 2011, les deux premiers cycles de l'éducation de base (Années d'études 1 à 6) sont classés dans l'enseignement primaire (CITE 1) et le troisième cycle dans le premier cycle de l'enseignement secondaire (CITE 2). En fonction du programme d'études national, l'école doit contribuer à former les jeunes pour qu'ils soient des individus créatifs et équilibrés, capables de bien fonctionner dans les différentes sphères de la vie telles que la famille et la société. (Années d'études 1 à 6 : CITE-P/CITE-A : 100 ; Années d'études 7 à 9 : CITE-P/CITE-A : 244)

Fidji - École primaire. L'école primaire débute à 6 ans et couvre huit années d'études (Années 1 à 8). Le programme ne se subdivise pas en cycles au niveau national. Dans le cadre de la soumission internationale de données, ce programme est considéré comme couvrant les niveaux 1 et 2 de la CITE. Les six premières années d'études sont classées dans la CITE 1 et les deux dernières années d'études sont classées dans la CITE 2. (Années d'études 1 à 6 : CITE-P/ CITE-A : 100 ; Années d'études 7 à 8 : CITE-P/CITE-A : 244)

Finlande - Peruskoulu (École générale). L'école générale assure neuf ans d'éducation de base générale obligatoire (primaire et premier cycle du secondaire) à partir de 7 ans. Elle compte deux cycles : le cycle inférieur (Années d'études 1 à 6) et le cycle supérieur (Années d'études 7 à 9). Au cours des six premières années, c'est le plus souvent un enseignant principal par classe qui donne l'instruction. Conformément à la Loi relative à l'Éducation de base, l'objectif général de l'éducation de base est de favoriser le développement des élèves pour une participation humaine et éthiquement responsable dans la société et de leur enseigner les connaissances et les aptitudes nécessaires dans la vie. Les élèves qui achèvent les neuf ans d'école générale reçoivent la certification de fin d'éducation de base. Dans la CITE 2011, le cycle inférieur (Années d'études 1 à 6) est classé dans l'enseignement primaire (CITE 1) et le cycle supérieur dans le premier cycle du secondaire (CITE 2). (Années d'études 1 à 6 : CITE-P/CITE-A : 100 ; Années d'études 7 à 9 : CITE-P/ CITE-A : 244)

Islande - Grunnskolar (École obligatoire). En Islande, l'école obligatoire dure dix années (Années d'études 1 à 10). II n'existe pas de distinction entre le primaire et le premier cycle de l'enseignement secondaire au niveau national ; ils forment un seul niveau scolaire et se déroulent normalement dans le même établissement. Généralement, les Années d'études 1 à 7 sont considérées comme les années de primaire (CITE 1) et les Années d'études 8 à 10 comme les années du premier cycle de secondaire (CITE 2). Les élèves reçoivent une certification au terme de l'école obligatoire. (Années d'études 1 à 7 : CITE-P/CITE-A : 100 ; Années d'études 8 à 10 : CITE-P/CITE-A : 244)

Kenya - Enseignement primaire. L'enseignement primaire national cible les enfants à partir de 6 ans. II consiste en huit années d'études groupées en deux cycles : le premier cycle couvre les Années d'études 1 à 3 et le second cycle les Années d'études 4 à 8. Les Années d'études 1 à 6 sont classées dans l'enseignement primaire (CITE 1) et les Années d'études 7 à 8 dans le premier cycle de l'enseignement secondaire (CITE 2). (Années d'études 1 à $6:$ CITE-P/CITE-A : 100 ; Années d'études 7 à 8 : CITE-P /CITE-A : 244)

Malawi - Enseignement primaire. La durée de l'enseignement primaire est huit ans. L'âge officiel d'entrée est 6 ans. Dans le CITE 2011, les Années d'études 1 à 6 sont classées dans l'enseignement primaire (CITE 1) et les Années d'études 7 et 8 comme les deux premières années d'études du premier cycle de l'enseignement secondaire (CITE 2). Ces deux années d'études ajoutées aux premières années de l'enseignement secondaire constituent le premier cycle de l'enseignement secondaire. (Années d'études 1 à 6 : CITE-P/CITE-A : 100 ; Années d'études 7 à 8 : CITE-P/CITE-A : 242)

Oman - (Éducation de base). Le programme éducatif de base dure dix ans et débute à 6 ans. II s'organise en deux cycles : le premier cycle couvre les Années d'études 1 à 4 et le second cycle les Années d'études 5 à 10. Ces deux cycles sont suivis de deux années de post-éducation de base (enseignement secondaire). Dans la CITE 2011, les six premières 
années d'études sont classées dans la CITE 1 et les quatre autres années d'études dans la CITE 2. (Années d'études 1 à 6 : CITE-P/CITE-A : 100 ; Années d'études 7 à 10 : CITE-P/CITE-A : 244)

République arabe syrienne - (Éducation de base). Le programme éducatif de base dure neuf ans (Années d'études 1 à 9) et débute à 6 ans. II s'organise en deux cycles : le cycle 1 couvre les quatre premières années d'études (1 à 4) et le cycle 2 couvre les Années d'études 5 à 9 . Après un achèvement complet, les élèves reçoivent la certification de fin de l'éducation de base et peuvent accéder au deuxième cycle de l'enseignement secondaire. Dans la CITE 2011, le premier cycle est classé dans la CITE 1 et le second dans la CITE 2. (Années d'études 1 à 4 : CITE-P/CITE-A : 100 ; Années d'études 5 à 9 : CITE-P/CITE-A : 244)

Timor oriental - Ensino basico Filial (Éducation de base). L'éducation de base couvre les neuf premières années de la scolarité. Elle consiste en trois cycles. Le premier cycle couvre les quatre premières années d'études, le second cycle comprend les Années d'études 5 et 6 , et le troisième cycle couvre les trois dernières années d'études. À des fins de comparabilité internationale, les deux premiers cycles (Années d'études 1 à 6) sont classés dans l'enseignement primaire et le dernier cycle (Années d'études 7 à 9) est considéré comme appartenant au premier cycle de l'enseignement général secondaire. (Années d'études 1 à 6 : CITE-P/CITE-A : 100 ; Années d'études 7 à 9 : CITE-P/CITE-A : 244)

\section{Exemples Groupe 6 :}

Programmes du niveau 1 de la CITE insuffisants pour un achèvement de niveau, avec donc un niveau d'éducation atteint correspondant à certaines composantes de l'enseignement primaire (sans achèvement de niveau) [CITE-P : 100 ; CITE-A : 030]

Éthiopie - Programme d'Éducation de base alternative (EBA) Le programme a été conçu comme une méthode accélérée pour atteindre l'enseignement primaire universel. L'EBA a le même âge d'entrée que l'enseignement primaire normal (6 ans), mais il propose un programme d'études qui ramène les quatre premières années d'études à trois. Lorsqu'ils réussissent la troisième année d'études, les élèves accèdent à l'Année d'études 5 de l'enseignement primaire normal. (CITE-P : 100/CITE-A : 030)

\section{Notes}

1. Les numéros de paragraphe constituent des références à la classification CITE 2011. Pour plus de détails, veuillez consulter le Guide du lecteur.

2. L'Institut de statistique de I'UNESCO (ISU) et I'Union européenne (UE) utilisent « République de Corée » comme dénomination officielle pour la « Corée ".

3. L'Institut de statistique de I'UNESCO (ISU) et l'Union européenne (UE) utilisent « Slovaquie » comme dénomination officielle pour la "République slovaque ». 


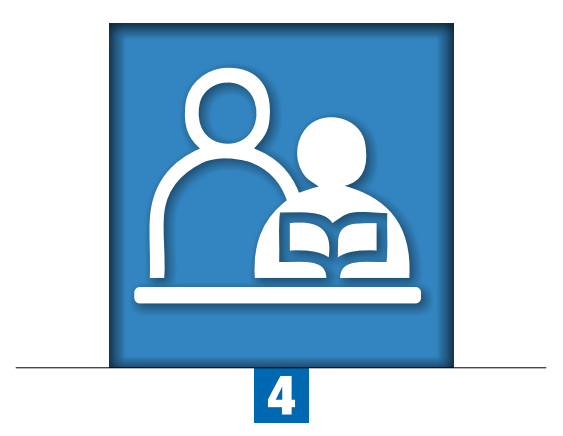

Chapitre 4

\section{Niveau 2 de la CITE 2011:}

\section{Premier cycle de l'enseignement secondaire}

Les programmes du premier cycle de l'enseignement secondaire sont destinés à poser les fondements d'un vaste ensemble de matières et à préparer les enfants et les jeunes à des études plus spécialisées dans le deuxième cycle de l'enseignement secondaire et à des niveaux d'enseignement plus élevés. Le début - ou la fin - du premier cycle de l'enseignement secondaire implique souvent un changement d'établissement pour les jeunes élèves, ainsi qu'un changement dans le type d'instruction.

Les programmes classés au niveau 2 de la CITE peuvent être désignés de plusieurs manières, par exemple : école secondaire ou collège (cycle 1/ premières années d'études), école secondaire inférieure, école moyenne ou junior high school. Si un programme couvre les niveaux 1 et 2 de la CITE, les expressions " enseignement fondamental " ou " enseignement de base » (second cycle/dernières années d'études du programme) sont souvent utilisées. En vue de la comparabilité internationale, l'expression " premier cycle de l'enseignement secondaire » est utilisée afin de qualifier le niveau 2 de la CITE. 


\section{DESCRIPTION}

\section{Définition}

$(\$ 139)^{1}$ Les programmes du niveau 2 de la CITE, ou « premier cycle de l'enseignement secondaire ", sont généralement destinés à compléter les acquis scolaires du niveau 1 de la CITE. Dans la plupart des cas, l'objectif est d'établir la base d'un apprentissage tout au long de la vie et d'un développement humain que les systèmes éducatifs pourront enrichir par de nouvelles possibilités d'éducation. Certains systèmes éducatifs peuvent offrir des programmes d'enseignement professionnel du niveau 2 de la CITE afin d'enseigner des compétences pertinentes pour le marché du travail.

(§140) À ce niveau, les programmes s'organisent généralement en une structure davantage orientée vers les matières enseignées et introduisent des concepts théoriques sur une variété de sujets. Les enseignants possèdent pour la plupart des qualifications pédagogiques dans des matières spécifiques. Plus fréquemment que dans le niveau 1 de la CITE, une classe d'élèves peut avoir plusieurs enseignants disposant chacun de connaissances spécialisées dans les matières qu'ils enseignent.

\section{Critères de classification (\$143)}

\section{Critères principaux}

a) Point de transition vers un enseignement davantage orienté vers les matières

(§144) La limite entre les niveaux 1 et 2 de la CITE coïncide avec le [premier] point de transition à partir duquel l'enseignement orienté vers les matières est privilégié dans les systèmes éducatifs.

b) Critères d'admission

(§145) Ce niveau impose l'achèvement du niveau 1 de la CITE ou la capacité d'étudier un contenu de niveau 2 de la CITE au travers d'un enseignement préalable et d'expériences personnelle et professionnelle. II est possible qu'un achèvement complet du niveau 1 de la CITE ou un niveau de réussite spécifique soit exigé pour avoir accès à certains ou à la totalité des programmes du niveau 2 de la CITE.

c) Durée cumulée depuis le début du niveau 1 de la CITE

(§146) Le niveau 2 de la CITE prend fin après 8 à 11 ans d'enseignement après le début du niveau 1 de la CITE.

\section{Critères subsidiaires}

a) Âge normal d'entrée

(§141) Le niveau 2 de la CITE commence après quatre à sept ans d'enseignement de niveau 1 de la CITE. Les élèves accèdent généralement au niveau 2 de la CITE lorsqu'ils ont entre 10 et 13 ans.

b) Qualifications des enseignants et organisation de l'enseignement par enseignants spécialisés

(§147) Le type de qualification des enseignants peut être différent si l'on compare le niveau 2 de la CITE au niveau 1 de la CITE. Les enseignants du niveau 2 de la CITE sont souvent spécialisés dans une ou plusieurs matières, ainsi qu'en pédagogie. De plus, l'organisation de l'enseignement peut différer de celle du niveau 1 de la CITE puisqu'il y a plus souvent plusieurs enseignants pour une classe qui enseignent chacun son ou ses domaines de spécialisation.

c) Relation avec la scolarité obligatoire

(§148) Dans de nombreux systèmes éducatifs caractérisés par une scolarité obligatoire légalement instituée, la fin du premier cycle de l'enseignement secondaire coïncide avec la fin de l'enseignement (général) obligatoire. 


\section{Dimensions complémentaires}

(§152) Deux dimensions permettent de distinguer les programmes éducatifs du niveau 2 de la CITE :

(§153) Orientation du programme :

- Général ; et

- Professionnel.

(§154) Achèvement de niveau :

- Non achèvement du niveau 2 de la CITE : programmes terminaux (ou succession de programmes) courts d'une durée de moins de deux ans appartenant au niveau 2 de la CITE ou qui se terminent après moins de huit années en durée cumulée d'enseignement depuis le début du niveau 1 de la CITE.

- Achèvement partiel du niveau 2 de la CITE : programmes qui représentent une durée d'au moins deux ans dans le niveau 2 de la CITE et une durée cumulée d'au moins huit années depuis le début du niveau 1 de la CITE. Ces programmes appartiennent à une succession de programmes de niveau 2 de la CITE sans toutefois constituer le dernier programme de cette succession à ce niveau.

- Achèvement du niveau 2 de la CITE sans accès direct à des niveaux plus élevés de la CITE : programmes d'une durée d'au moins deux ans au niveau 2 de la CITE qui se terminent après au moins huit années en durée cumulée d'enseignement depuis le début du niveau 1 de la CITE mais qui ne donnent pas un accès direct au niveau 3 de la CITE.

- Achèvement du niveau 2 de la CITE avec accès direct à un niveau plus élevé de la CITE : tout programme donnant un accès direct au niveau 3 de la CITE quelle que soit sa durée dans le niveau ou sa durée cumulée depuis le début du niveau 1 de la CITE.

\section{CORRESPONDANCES ENTRE LA CITE 2011 ET LA CITE 1997}

La couverture du niveau 2 de la CITE est identique dans les révisions 1997 et 2011 de la CITE.

Les dimensions complémentaires du niveau 2 de la CITE sont différentes dans la dernière version de la CITE. (§278)

a) Orientation du programme

La CITE 1997 définit trois types d'orientation : général, préprofessionnel et professionnel. La CITE 2011 ne propose que deux orientations au niveau 2 de la CITE : général et professionnel. Les programmes classés au préalable dans la catégorie préprofessionnelle (CITE 1997) ne permettent pas l'obtention de certifications pertinentes pour le marché du travail et de ce fait sont maintenant classés pour la plupart dans l'enseignement général.

b) Achèvement de niveau et programme de destination

La CITE 1997 classe les programmes en fonction des programmes et des niveaux auxquels ils devaient donner accès (le programme de destination). Au niveau 2 de la CITE, trois destinations étaient définies :

- Les programmes A, donnant accès au deuxième cycle de l'enseignement secondaire, aux niveaux 3A ou 3B de la CITE 1997 (programmes avec accès direct à l'enseignement supérieur) ;

- Les programmes B, donnant accès au deuxième cycle de l'enseignement secondaire, au niveau 3C, mais pas aux niveaux 3A ou 3B de la CITE 1997 (programmes avec accès à l'enseignement post-secondaire non-supérieur seulement, ou au marché du travail); et

- Les programmes C, sans accès direct au deuxième cycle de l'enseignement secondaire (c'est-à-dire des programmes terminaux menant au marché du travail).

La CITE 2011 classe les programmes selon le type d'achèvement du niveau et l'accès à d'autres programmes et niveaux. Les destinations A et B de la CITE 1997 ont été assimilées en une seule catégorie dans la CITE 2011 : achèvement (complet) du niveau 2 de la CITE 2011 avec accès direct au deuxième cycle de l'enseignement secondaire. La destination C de la CITE 1997 se subdivise en trois catégories dans la CITE 2011 :

- achèvement (complet) du niveau 2 de la CITE 2011 sans accès direct au deuxième cycle de l'enseignement secondaire ;

- achèvement partiel du niveau 2 de la CITE 2011 sans accès direct au deuxième cycle de l'enseignement secondaire ; et

- insuffisant pour un achèvement ou un achèvement partiel du niveau 2 de la CITE 2011. 


\section{DIRECTIVES POUR LA CLASSIFICATION DES PROGRAMMES ET DE LEURS CERTIFICATIONS AU NIVEAU 2 DE LA CITE}

Les programmes du premier cycle de l'enseignement secondaire sont destinés à poser les fondements d'un vaste ensemble de matières et à préparer les enfants et les jeunes à des études plus spécialisées du second cycle de l'enseignement secondaire et à des niveaux d'enseignement plus élevés. Le début - ou la fin - du premier cycle de l'enseignement secondaire implique souvent un changement d'établissement pour les jeunes élèves, ainsi qu'un changement dans le type d'instruction. Les programmes du premier cycle du secondaire se fondent sur l'enseignement primaire et comprennent généralement des cours davantage orientés vers les matières enseignées qu'au niveau primaire. Généralement, tous les élèves suivent le même programme d'études et ont peu ou pas de choix concernant les matières à étudier. Lorsqu'ils terminent le premier cycle de l'enseignement secondaire, les jeunes sont supposés avoir une base solide dans les matières fondamentales (généralement la lecture et l'écriture, les mathématiques, l'histoire, la géographie, les sciences et souvent une langue étrangère ou une seconde langue ; l'art, la musique et l'éducation physique peuvent aussi faire partie du programme d'études).

Dans certains systèmes éducatifs, les programmes peuvent se différencier par leur orientation, bien que cela soit plus fréquent dans le second cycle de l'enseignement secondaire.

Les programmes professionnels, quand ils existent à ce niveau, offrent généralement des options aux jeunes qui souhaitent se préparer à une entrée directe sur le marché du travail dans des emplois peu ou semi-qualifiés (Exemples Groupe 1). Ils peuvent aussi constituer la première étape de l'enseignement professionnel, donnant accès à des programmes professionnels plus avancés dans le second cycle de l'enseignement secondaire (Exemples Groupe 2).

Dans la plupart des systèmes éducatifs, la grande majorité des élèves sont inscrits dans des programmes généraux du premier cycle du secondaire destinés à les mener vers le deuxième cycle de l'enseignement secondaire (Exemples Groupe 3).

Le niveau 2 de la CITE comprend également les programmes éducatifs pour adultes, dont le contenu est similaire à celui de l'enseignement délivré à ce niveau (par exemple des programmes permettant aux adultes d'acquérir les compétences fondamentales nécessaires à la poursuite de l'apprentissage), et des programmes éducatifs pour les personnes ayant des besoins éducatifs spéciaux (par exemple des handicaps, des difficultés ou des désavantages) (Exemples Groupe 4).

Il existe aussi des programmes de seconde chance ou de réintégration qui révisent les matières déjà abordées dans les programmes du premier cycle du secondaire ou offrent des options à des jeunes qui sinon risqueraient de quitter l'école sans certification (Exemples Groupe 5). Ils peuvent aussi offrir aux jeunes des opportunités de changer de filière ou d'accéder à une profession nécessitant une certification de fin de premier cycle du secondaire à laquelle leurs études en cours ne leur donnent pas droit. Ces programmes sont classés dans le niveau 2 de la CITE (\$156).

À ce niveau, il existe également des programmes visant l'intégration des migrants - adultes et jeunes (Exemples Groupe 6).

Par le passé, la fin de ce niveau coïncidait souvent avec la fin de la scolarité obligatoire ; aujourd'hui, de plus en plus de pays étendent la scolarité obligatoire jusqu'au deuxième cycle de l'enseignement secondaire. Les programmes du premier cycle du secondaire durent entre deux et cinq ans, trois ans étant la durée la plus fréquente. Les programmes de ce niveau peuvent être plus courts dans certains pays où il peut exister une succession de programmes dans le niveau. Les programmes séquentiels sont moins communs dans le premier cycle du secondaire que dans le deuxième cycle du secondaire, mais ils peuvent exister en cas de transfert d'un établissement à l'autre pendant les études de ce niveau - par exemple quand les écoles de base couvrent huit années d'études ou plus au-delà du niveau primaire.

\section{Programmes couvrant le premier cycle de l'enseignement secondaire et un autre niveau de la CITE}

II faut être particulièrement attentif aux programmes couvrant le premier cycle de l'enseignement secondaire et les niveaux précédents ou postérieurs. Dans ces cas, seuls les années d'études, phases ou cycles correspondant aux critères des niveaux respectifs (niveau 1, 2 ou 3 de la CITE) doivent être classés dans ces niveaux. (\$149) 


\section{NIVEAU D'ÉDUCATION ATTEINT AU NIVEAU 2 DE LA CITE}

À l'instar de l'enseignement primaire, les programmes du premier cycle du secondaire ne mènent pas toujours à une certification finale reconnue - surtout quand le premier et le deuxième cycle de l'enseignement secondaire sont proposés dans le même établissement ou quand la fin du premier cycle de l'enseignement secondaire ne coïncide pas avec la fin de la scolarité obligatoire. Dans ce cas, d'autres formes de reconnaissance formelle de l'achèvement complet du programme ou du niveau peuvent être utilisées en vue de déterminer le niveau d'éducation atteint. II peut s'agir d'une confirmation formelle de fréquentation pendant l'entièreté de la dernière année du programme ou le droit d'accéder à des programmes ou des niveaux plus élevés.

En vue de mesurer le niveau d'éducation atteint, la CITE 2011 opère une distinction entre achèvement complet et partiel du premier cycle de l'enseignement secondaire, bien que l'achèvement partiel soit rare à ce niveau.

La CITE subdivise les programmes menant à un achèvement complet du premier cycle de l'enseignement secondaire entre ceux donnant accès au deuxième cycle de l'enseignement secondaire et ceux ne le donnant pas. Les deux sont toutefois considérés comme un achèvement du premier cycle du secondaire. Ce dernier groupe comprend habituellement des programmes professionnels qui sont souvent destinés à des élèves plus âgés que l'âge théorique pour le premier cycle de l'enseignement secondaire.

L'achèvement partiel d'un niveau a une signification très spécifique et n'est pas synonyme d' "études incomplètes » ou d' "études limitées » à ce niveau. Un programme menant à l'achèvement partiel d'un niveau est un programme décernant une certification reconnue ou une autre confirmation formelle octroyée par les autorités nationales compétentes pour l'éducation en reconnaissance de l'achèvement complet du programme. Ce programme appartient à une succession de programmes de niveau 2 de la CITE sans toutefois constituer le dernier programme de cette succession à ce niveau. Le programme doit également représenter au moins huit années en durée cumulée d'enseignement depuis le début de l'enseignement primaire et au moins deux années d'enseignement (cumulé) dans le premier cycle de l'enseignement secondaire (Exemples Groupe 7). Veuillez noter que l'achèvement partiel d'un niveau est également différent de la souscatégorie 'non achèvement du niveau'. Cette dernière s'applique à des programmes ne satisfaisant pas les exigences de durée pour un achèvement partiel ou complet de niveau et menant donc à un niveau d'éducation atteint inférieur à celui du programme (voir Exemples Groupe 8).

Dans le premier cycle du secondaire, l'achèvement partiel d'un niveau se produit le plus souvent en cas de changement d'établissement au cours du niveau. Dans le cadre du processus de transition d'un établissement à un autre, les pays peuvent organiser des évaluations ou des examens formels qui mesurent les progrès des élèves et mènent à la reconnaissance de l'achèvement complet du programme d'enseignement national concerné.

(§159) En ce qui concerne le niveau d’éducation atteint, les certifications reconnues des programmes du niveau 2 de la CITE considérés comme insuffisants pour être pris en compte comme un achèvement ou un achèvement partiel du niveau 2 de la CITE sont classées dans le niveau 1 de la CITE.

(§160) De même, les certifications reconnues des programmes du niveau 3 de la CITE considérés comme insuffisants pour être pris en compte comme un achèvement ou un achèvement partiel du niveau 3 de la CITE sont classées dans le niveau 2 de la CITE.

Si le programme ne mène pas à des certifications reconnues (ou si l'achèvement complet n'est pas reconnu formellement d'une autre façon par les autorités nationales compétentes), le concept d'achèvement partiel d'un niveau ne s'applique pas. Dans ce cas, le niveau d'éducation atteint de niveau 2 de la CITE ne peut être appliquée qu'à la fin de la succession complète de programmes, lorsque la certification ou la confirmation formelle correspondante marquant l'achèvement complet est obtenue.

Les programmes du niveau 2 de la CITE qui ne donnent pas un accès direct au niveau 3 de la CITE et représentent un nombre d'années d'études moindre, soit depuis le début de l'enseignement primaire (c'est-à-dire moins de huit ans) soit dans le premier cycle de l'enseignement secondaire (c'est-à-dire moins de deux ans) ou les deux, sont considérés comme insuffisants pour un achèvement partiel ou complet du premier cycle de l'enseignement secondaire, et font que le niveau d'éducation atteint est l'enseignement primaire (Exemples Groupe 8). 
Les utilisateurs de la classification souhaitant répartir les résultats selon un ensemble de catégories de niveau d'éducation atteint plus simplifié que cela n'est acceptable dans la CITE-A peuvent se voir dans l'obligation de décider comment traiter l'achèvement partiel d'un niveau. Selon le principal objectif des analyses à effectuer, deux approches différentes sont possibles :

(i) Certains utilisateurs voudraient savoir quel pourcentage de la population concernée a achevé chaque niveau d'enseignement de la CITE avec succès. Pour cela, ils doivent classer l'achèvement partiel d'un niveau au niveau inférieur au niveau d'études (c'est-à-dire au niveau primaire dans le cas de l'achèvement partiel du premier cycle de l'enseignement secondaire).

(ii) D'autres utilisateurs voudraient mesurer au moins certaines études réussies et reconnues à un niveau donné notamment lorsque cela représente un point de transition important dans le système éducatif ou la première obtention d'un diplôme dans un niveau de la CITE donné. Pour cela, ils doivent classer l'achèvement partiel d'un niveau au niveau CITE du niveau d'éducation (c'est-à-dire au niveau du premier cycle de secondaire en cas d'achèvement partiel du premier cycle de l'enseignement secondaire).

Les deux approches sont valables. II incombe à l'utilisateur/l'analyste de décider de l'approche la plus appropriée en fonction des circonstances. II est recommandé que le traitement de l'achèvement partiel utilisé pour le calcul des indicateurs sur le niveau d'éducation atteint soit indiqué par des métadonnées accompagnant ces statistiques.

\section{EXEMPLES DE PROGRAMMES ET DE CERTIFICATIONS CLASSÉS AU NIVEAU 2 DE LA CITE}

(§142) Les programmes destinés à être classés au niveau 2 de la CITE peuvent être désignés de plusieurs manières, par exemple : école secondaire ou collège (cycle 1/premières années d'études), école secondaire inférieure, école moyenne ou junior high school. Si un programme couvre les niveaux 1 et 2 de la CITE, les expressions " enseignement fondamental " ou " enseignement de base " (seconde phase /dernières années d'études du programme) sont souvent utilisées. En vue de la comparabilité internationale, l'expression « premier cycle de l'enseignement secondaire » est utilisée afin de qualifier le niveau 2 de la CITE.

\section{Exemples Groupe 1:}

Programmes professionnels destinés aux jeunes souhaitant se préparer à une entrée directe sur le marché du travail dans des emplois peu ou semi-qualifiés [CITE-P : 251 ou 253 ; CITE-A : 100 ou 253]

Tunisie - (Certificat de compétence). L'accès à ce programme exige l'achèvement de l'Année d'études 7, la première année d'études du premier cycle de l'enseignement secondaire. II s'agit d'un programme d'enseignement professionnel de deux ans menant au Certificat de compétence, qui donne uniquement accès au marché du travail. (CITE-P/CITE-A : 253)

\section{Exemples Groupe 2 :}

Programmes professionnels préparant à une entrée directe sur le marché du travail mais donnant également accès aux programmes du deuxième cycle du secondaire [CITE-P : 254 ; CITE-A : 254]

Bulgarie - Profesionalni programi- parva stepen na profesionalna kvalifikatsiya sled 6 ili 7 klas (Programme professionnel pour une certification professionnelle de premier niveau après achèvement de l'Année d'études 6 ou 7). Les élèves accèdent à ce programme après avoir achevé deux années au niveau 2 de la CITE. Le programme dure deux à trois ans et mène à deux certifications : réussite de l'éducation de base (donnant accès au niveau 3 de la CITE) et certification professionnelle de premier niveau. (CITE-P/CITE-A : 254)

Liban - (Aptitude professionnelle). Ce programme professionnel de deux ans exige au minimum l'achèvement de la première année du premier cycle de l'enseignement secondaire. Après un achèvement complet, les sortants se voient attribuer le Certificat d'aptitude professionnelle, qui ouvre les portes du marché du travail et donne accès à des niveaux d'enseignement plus élevés. (CITE-P/CITE-A : 254)

Pays-Bas - WEB-assistentenopleiding (Enseignement professionnel : formation au niveau d'assistant (niveau 1)). II s'agit de programmes éducatifs à temps plein ou partiel se déroulant à l'école ou en système double et destinés aux élèves de 16 ans et plus. (Les programmes à temps partiel sont uniquement accessibles aux élèves de 18 ans et plus.) Les 
programmes à temps plein dure généralement un an et débouche sur le MBO niv. 1: assistent (Certificat d'enseignement professionnel secondaire, niveau assistant). Les personnes ayant achevé avec succès le programme peuvent poursuivre leurs études dans le deuxième cycle de l'enseignement secondaire, mais la plupart d'entre eux entrent sur le marché du travail. (CITE-P/CITE-A : 254)

\section{Exemples Groupe 3 : \\ Programmes généraux donnant accès au deuxième cycle de l'enseignement secondaire [CITE-P : 244 ; CITE-A : 244]}

Australie - École secondaire : 1er cycle. Le premier cycle de l'école secondaire dure trois ou quatre ans, selon la durée de l'école primaire dans l'état concerné, et se termine par l'octroi du Certificat de l'Année 10. Les élèves suivent un programme scolaire général qui donne l'opportunité de poursuivre ses études. (CITE-P/CITE-A : 244)

Égypte - (Enseignement préparatoire). II s'agit d'un programme post-primaire général de trois ans. Lors de son achèvement, les élèves reçoivent le certificat d'éducation de base. Les élèves peuvent ensuite accéder à des programmes éducatifs généraux ou professionnels du second cycle de l'enseignement secondaire. (CITE-P/CITE-A : 244)

Allemagne - Sekundarbereich I mit Qualifikation für weiterführende Bildungsgänge (Écoles du premier cycle du secondaire donnant accès aux programmes généraux du deuxième cycle du secondaire). Ce programme (Années d'études 5 à 9 ou 10) qui succède aux quatre années de l'école primaire se caractérise par le commencement d'un programme d'études davantage axé sur les matières. Les personnes qui achèvent le programme avec succès ont le droit de poursuivre leurs études dans les établissements du deuxième cycle du secondaire général (Gymnasiale Oberstufe) qui préparent les élèves aux programmes de la CITE 6 ou 7. (CITE-P/CITE-A : 244)

Inde - Deuxième cycle de primaire (Années d'études VI à VIII). L'accès au programme éducatif général de trois ans exige un certificat de primaire, décerné à l'achèvement complet du programme de l'enseignement primaire de six ans (CITE 1). Les élèves ayant achevé le deuxième cycle de l'enseignement primaire reçoivent le certificat du deuxième cycle de primaire, qui donne accès au programme du deuxième cycle de secondaire (CITE 3). (CITE-P/CITE-A : 244)

Slovénie - Osnovnošolsko izobraževanje (7.-9. razred) (Éducation de base (Années d'études 7 à 9)). II s'agit d'un programme de trois ans menant au Zaključno spričevalo (osnovne šole) (Certificat d'achèvement de l'école de base obligatoire) qui donne un accès direct à des programmes du deuxième cycle de l'enseignement secondaire. En général, les élèves débutent le programme à 12 ans, après avoir achevé l'enseignement primaire avec succès. (CITE-P /CITE-A : 244)

Îles Salomon - Junior Secondary Education (Premier cycle de l'enseignement secondaire, Années 1-3) II s'agit d'un programme éducatif général de trois ans qui débute après l'achèvement complet du Standard 6 (qui marque la fin de l'enseignement primaire). Au terme du programme, les élèves passent le National Form 3 Examination (NF3) donnant accès au deuxième cycle de l'enseignement secondaire. (CITE-P/CITE-A : 244)

\section{Exemples Groupe 4 :}

Éducation des adultes enseignant les compétences fondamentales nécessaires à la poursuite de l'apprentissage et programmes du niveau 2 de la CITE pour les élèves ayant des besoins éducatifs spéciaux [CITE-P : 243, 244, 253 ou 254 ; CITE-A : 243, 244, 253 ou 254]

Belgique (Communauté flamande) - Buitengewoon secundair onderwijs - opleidingsvorm 1 en 2 (Enseignement secondaire spécial - types 1 et 2). Ce programme s'adresse aux enfants ayant besoin d'une aide spéciale, temporairement ou de manière permanente. Dans l'enseignement secondaire spécial, l'enseignement s'organise en fonction du type de handicap et des possibilités de l'élève. L'enseignement de type 1 fournit un enseignement social visant l'intégration de l'élève dans un environnement protégé. L'enseignement de type 2 fournit un enseignement général et social visant l'intégration de l'élève dans un environnement de vie et de travail protégé. (CITE-P/CITE-A : 253)

Allemagne - Sekundarbereich I, Abendschulen (Cours du soir du premier cycle du secondaire). Ce programme (d'une durée d'un ou deux ans) est destiné aux adultes n'ayant aucune certification du niveau 2 de la CITE ou une certification faible (par exemple Hauptschulabschluss) et souhaitant obtenir une certification plus élevée du premier cycle du secondaire (le plus souvent le Mittlerer Schulabschluss). (CITE-P/CITE-A : 244) 
Koweit - (Cycle intermédiaire pour adultes). Ce programme éducatif formel pour adultes cible normalement les adultes de 15 ans et plus ayant achevé l'enseignement primaire. À l'instar du cycle intermédiaire initial (premier cycle du secondaire), le programme a une durée théorique de quatre ans. Après un achèvement complet, les élèves peuvent accéder aux programmes éducatifs pour adultes du deuxième cycle du secondaire. (CITE-P/CITE-A : 244)

Mexique - Capacitación para el trabajo (Formation professionnelle du premier cycle du secondaire). La durée usuelle de ces programmes est de deux ans, bien qu'il existe également des programmes plus longs ou plus courts. Les élèves de ces programmes sont généralement des adultes. Le programme vise à former des personnes (15 ans et plus) en vue de leur donner accès au monde du travail. (CITE-P/CITE-A : 243)

\section{Exemples Groupe 5 :}

Programmes de seconde chance ou de réintégration pour les élèves n'ayant pas encore achevé le premier cycle de l'enseignement secondaire [CITE-P : 243, 244, 253 ou 254 ; CITE-A : 243, 244, 253 ou 254]

Irlande - Youthreach (Foundation Level). II s'agit d'un programme général de deux ans destiné aux personnes qui quittent l'enseignement sans certification. Le certificat est disponible dans un éventail de niveaux, y compris un Junior Certificate pour le premier cycle du secondaire. (CITE-P/CITE-A : 244)

Maroc - (Formation professionnelle : niveau spécialisation). Ce programme est accessible aux élèves en sixième année de l'enseignement primaire ayant au moins 15 ans. Ce programme de deux ans prépare les élèves à rejoindre le marché du travail. En outre, il donne accès aux programmes des niveaux d'éducation plus élevés (CITE 3). (CITE-P/CITE-A : 254)

République slovaque ${ }^{2}$ - Praktická škola (École pratique). II s'agit d'un programme général d'un à trois ans fournissant un enseignement et une formation à des activités professionnelles simples destinés à donner une seconde chance aux jeunes ayant déjà participé à un cycle complet du premier cycle de l'enseignement secondaire. Les entrants ont généralement 15 ans ou plus. Le contenu n'est pas suffisamment complexe pour être classé dans le second cycle de l'enseignement secondaire. II mène au Vysvedčenie (Certificat). (CITE-P/CITE-A : 243)

\section{Exemples Groupe 6 : \\ Programmes destinés à l'intégration des nouveaux migrants \\ [CITE-P : 241, 243, 244, 253 ou 254 ; CITE-A : 100, 243, 244, 253 ou 254]}

Belgique (Communauté flamande) - Onthaalklas voor anderstalige nieuwkomers (Classe d'accueil pour immigrants). L'enseignement d'accueil vise les primo-arrivants en Belgique parlant à peine le néerlandais. Le principal objectif est l'apprentissage du néerlandais et l'intégration des participants dans le type d'enseignement secondaire et le domaine d'étude les mieux adaptés possibles à leurs capacités individuelles. Le programme d'études de l'année d'accueil se concentre principalement sur l'acquisition du néerlandais. Une personne peut accéder à la classe d'accueil quand elle satisfait les critères suivants : 1) avoir au moins 12 ans et pas plus de 18 ans au 31 décembre après le début de l'année scolaire ; 2) être un primo-immigrant (au maximum un an de résidence en Belgique) ; 3) avoir une langue maternelle ou une langue parlée à domicile qui ne soit pas le néerlandais ; 4) ne pas avoir une connaissance suffisante du néerlandais pour suivre les cours dans l'enseignement général ; et 5) être inscrit depuis neuf mois au maximum dans un établissement ayant le néerlandais comme principale langue d'instruction. (CITE-P : 241 ; CITE-A : 100)

Luxembourg - Classes d'accueil (ACCU) (12-15 ans) et Classes d'insertion pour jeunes adultes (CLIJA) (16-17 ans). II s'agit de programmes généraux d'un an destinés à préparer les jeunes venant d'arriver de l'étranger à entrer dans le système scolaire normal, soit dans le premier cycle du secondaire (Classes d'accueil (ACCU) (12-15 ans)), soit dans le deuxième cycle de l'enseignement secondaire (Classes d'insertion pour jeunes adultes (CLIJA) (16-17 ans)). (CITE-P : 241/CITE-A : 100 et CITE-P/CITE-A : 244 respectivement)

Norvège - Introduksjonsordning og opplæring I norsk og samfunnskunnskap for nyankomne innvandrere (Programme d'initiation à la langue norvégienne et à la vie en société pour les nouveaux immigrants). Le programme dure jusqu'à deux ans et mène au Fullført introduksjonsprogram (Certificat de langue norvégienne et d'études civiques pour les immigrants adultes). II ne donne pas accès à des niveaux d'éducation plus élevés. (CITE-P : 241 ; CITE-A : 100) 


\section{Exemples Groupe 7 :}

Programmes et certifications ayant pour résultat l'achèvement partiel d'un niveau

[CITE-P : 242 ou 252 ; CITE-A : 242 ou 252]

Lituanie - Profesinio mokymo programa, skirta asmenims, neturintiems pagrindinio išsilavinimo (Programmes d'enseignement professionnel pour les personnes n'ayant pas d'éducation de base). Après l'achèvement de l'enseignement primaire, les élèves peuvent accéder à ce programme de deux ans qui conduit à un certificat de qualification professionnelle permettant aux élèves de poursuivre leur éducation dans d'autres programmes du premier cycle de l'enseignement secondaire qui, à leur terme, donnent accès au second cycle de l'enseignement secondaire. (CITE-P/CITE-A : 252)

\section{Exemples Groupe 8 :}

Programmes du niveau 2 de la CITE insuffisants pour un achèvement de niveau, avec donc un niveau d'éducation atteint correspondant au niveau 1 de la CITE [CITE-P : 241 ou 251 ; CITE-A : 100]

Algérie - (Formation professionnelle spécialisée). Ce programme d'un an est destiné à préparer les jeunes adolescents à un accès direct au marché du travail. Le programme est accessible aux jeunes âgés au moins de 11 ans capables de lire et d'écrire. À l'achèvement du programme, les élèves reçoivent un certificat de fin d'études, considéré localement comme supérieur à l'enseignement primaire mais inférieur au premier cycle du secondaire. Le programme est considéré comme insuffisant pour un achèvement de la CITE 2. (CITE-P : 251 ; CITE-A : 100)

Estonie - Põhihariduse nõudeta kutseõpe (Enseignement professionnel pour les personnes n'ayant pas d'éducation de base et ayant dépassé l'âge de fréquentation obligatoire de l'école). L'âge d'entrée dans ce programme va de 17 à 19 ans environ et sa durée est de six mois à deux ans et demi. Le critère d'admission à ce programme est l'achèvement de la CITE 1. Les élèves obtiennent un certificat de fin d'enseignement professionnel qui est considéré comme supérieur au niveau 1 de la CITE mais aussi comme insuffisant pour une classification comme une certification du niveau 2 de la CITE. (CITE-P : 251 ; CITE-A : 100)

\section{Notes}

1. Les numéros de paragraphe constituent des références à la classification CITE 2011. Pour plus de détails, veuillez consulter le Guide du lecteur.

2. L'Institut de statistique de I'UNESCO (ISU) et l'Union européenne (UE) utilisent « Slovaquie " comme dénomination officielle pour la "République slovaque». 



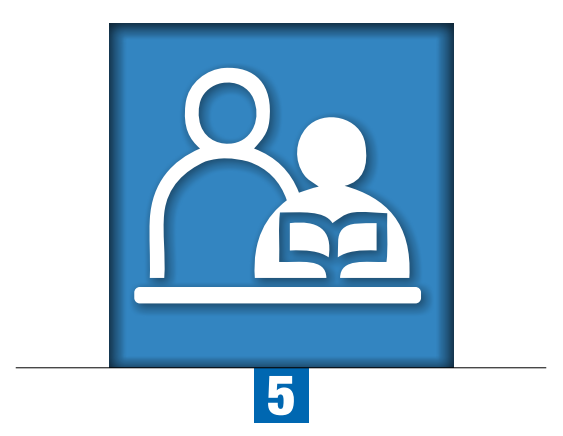

Chapitre 5

\section{Niveau 3 de la CITE 2011:}

\section{Deuxième cycle de l'enseignement secondaire}

Les programmes du deuxième cycle de l'enseignement secondaire sont plus spécialisés que ceux du premier cycle de l'enseignement secondaire. Ils proposent un éventail plus large d'options et de filières pour achever l'enseignement secondaire. L'éventail de matières étudiées par un élève est en général moins large que dans les niveaux d'éducation inférieurs, mais le contenu est plus complexe et l'étude plus approfondie. Les programmes proposés sont différenciés par orientation et souvent par larges groupes de matières.

Les programmes destinés à être classés au niveau 3 de la CITE peuvent être désignés de plusieurs manières, par exemple : école secondaire ou lycée (cycle 2/ dernières années d'études de l'enseignement secondaire). En vue de la comparabilité internationale, l'expression "deuxième cycle de l'enseignement secondaire » est utilisée afin de qualifier le niveau 3 de la CITE. 


\section{DESCRIPTION}

\section{Définition}

$(\$ 162)^{1}$ Les programmes du niveau 3 de la CITE, ou « deuxième cycle du secondaire ", sont généralement conçus pour compléter l'enseignement secondaire et préparer à l'enseignement supérieur, et/ou pour enseigner des compétences pertinentes pour exercer un emploi.

(§163) Les programmes de ce niveau offrent aux élèves un enseignement plus varié, spécialisé et approfondi que les programmes du niveau 2 de la CITE. Ils sont plus différenciés et proposent un éventail d'options et de filières plus large. Les enseignants sont souvent hautement qualifiés dans les matières qu'ils enseignent, surtout dans les dernières années d'études du niveau.

\section{Critères de classification $(\$ 166)$}

\section{Critères principaux}

a) Contenu

(§167) Les programmes qui constituent le deuxième ou le dernier cycle de l'enseignement secondaire peuvent être des programmes d'enseignement général ou professionnel. Certains de ces programmes donnent un accès direct au niveau 4, et/ou aux niveaux 5, 6 ou 7 de la CITE.

b) Critères d'admission

(§168) Le niveau 3 de la CITE exige l'achèvement du premier cycle de l'enseignement secondaire (niveau 2 de la CITE) ou la capacité de maîtriser un contenu du niveau 3 de la CITE par une combinaison d'enseignement préalable et d'expériences personnelles et professionnelles. II est possible qu'une certification marquant l'achèvement du niveau 2 de la CITE ou un niveau de réussite spécifique soit exigée pour avoir accès à certains ou à la totalité des programmes du niveau 3 de la CITE.

c) Durée cumulée depuis le début du niveau 1 de la CITE

(\$164) Le niveau 3 de la CITE commence après 8 à 11 ans d'enseignement depuis le début du niveau 1 de la CITE. L'âge d'admission à ce niveau est normalement de 14 à 16 ans. Les programmes du niveau 3 de la CITE prennent généralement fin 12 à 13 ans après le commencement du niveau 1 de la CITE (ou vers 17 ou 18 ans), 12 ans étant la durée cumulée la plus répandue. Toutefois, la sortie du deuxième cycle de l'enseignement secondaire peut se produire après 11 à 13 ans d'enseignement depuis le début du niveau 1 de la CITE selon les systèmes éducatifs.

\section{Critères subsidiaires}

a) Point de transition du programme

(§169) La transition du niveau 2 au niveau 3 de la CITE coïncide avec le point de transition où les programmes offrent aux élèves un enseignement plus varié, spécialisé et approfondi dans des matières ou domaines spécifiques. En règle générale, les programmes sont plus différenciés et proposent un éventail d'options et de filières plus large.

b) Qualifications des enseignants

(\$170) Les normes relatives aux qualifications des enseignants peuvent différer si l'on compare les niveaux 2 et 3 de la CITE. Outre une formation pédagogique, les enseignants peuvent être plus qualifiés dans la matière qu'ils enseignent. 


\section{Dimensions complémentaires}

(§174) Deux dimensions permettent de distinguer les programmes éducatifs du niveau 3 de la CITE.

(§175) Orientation du programme :

- Général ; et

- Professionnel.

(§176) Achèvement de niveau :

- Non achèvement du niveau 3 de la CITE : programmes terminaux (ou succession de programmes) courts d'une durée de moins de deux ans appartenant au niveau 3 de la CITE ou qui se terminent après moins de onze années en durée cumulée d'enseignement depuis le début du niveau 1 de la CITE.

- Achèvement partiel du niveau 3 de la CITE : programmes qui représentent une durée d'au moins deux ans dans le niveau 3 de la CITE et une durée cumulée d'au moins onze années depuis le début du niveau 1 de la CITE. Ces programmes appartiennent à une succession de programmes de niveau 3 de la CITE sans toutefois constituer le dernier programme de cette succession à ce niveau.

- Achèvement du niveau 3 de la CITE sans accès direct aux premiers programmes de l'enseignement supérieur aux niveaux 5, 6 ou 7 de la CITE : programmes d'une durée minimale de deux ans au niveau 3 de la CITE et finissant après au moins 11 ans d'enseignement en durée cumulée depuis le début du niveau 1 de la CITE. Ces programmes peuvent être terminaux ou donner uniquement un accès direct au niveau 4 de la CITE.

- Achèvement du niveau 3 de la CITE avec accès direct aux premiers programmes de l'enseignement supérieur aux niveaux 5, 6 ou 7 de la CITE : tout programme donnant un accès direct aux niveaux 5, 6 ou 7 de la CITE quelle que soit sa durée au niveau 3 ou sa durée cumulée depuis le début du niveau 1 de la CITE.

(§178) Ce niveau peut aussi inclure certains programmes d'enseignement professionnel de second cycle dans les cas où les points de transition en fin de second cycle correspondent aux points de transition entre niveaux dans d'autres parcours, surtout de l'enseignement général, offerts par le système.

(\$179) Ce niveau comprend aussi les programmes de seconde chance ou de réintégration du deuxième cycle du secondaire. Ces programmes s'adressent en général à des individus qui :

- ont cessé leur éducation avant d'avoir mené à terme le deuxième cycle du secondaire, leur permettant ainsi de réintégrer le système éducatif et d'achever le deuxième cycle du secondaire ; ou

- ont achevé le deuxième cycle de l'enseignement secondaire mais qui souhaitent accéder à un programme ou à une profession pour lesquels ils ne sont pas encore certifiés.

\section{CORRESPONDANCES ENTRE LA CITE 2011 ET LA CITE 1997}

La couverture du niveau 3 de la CITE est pour ainsi dire identique dans les versions 1997 et 2011 de la CITE, avec l'exception suivante dans le niveau 4 de la CITE 1997 :

(§190) Les programmes conçus pour réviser le contenu des programmes du niveau 3 de la CITE - par exemple, dans le but de préparer les élèves aux examens d'entrée dans l'enseignement supérieur - doivent être inclus dans le niveau 3 de la CITE [2011].

Les dimensions complémentaires du niveau 3 de la CITE sont différentes dans la dernière version de la CITE. (§278)

\section{a) Orientation du programme}

La CITE 1997 définit trois types d'orientation : général, préprofessionnel et professionnel. La CITE 2011 ne propose que deux orientations au niveau 3 de la CITE : général et professionnel. Les programmes classés au préalable dans la catégorie préprofessionnelle (CITE 1997) ne permettent pas l'obtention de certifications pertinentes pour le marché du travail et de ce fait sont maintenant classés pour la plupart dans l'enseignement général. 
b) Achèvement de niveau et programme de destination

La CITE 1997 classait les programmes en fonction des programmes et des niveaux auxquels ils devaient donner accès (le programme de destination). Au niveau 3 de la CITE, trois destinations étaient définies :

- Les programmes A, donnant accès à l'enseignement supérieur, au niveau 5A de la CITE 1997 (c'est-à-dire les programmes avec un accès direct à des programmes de niveau doctorat ou équivalent) ;

- Les programmes B, donnant accès à l'enseignement supérieur au niveau 5B, mais pas au niveau 5A, de la CITE 1997 (c'est-à-dire les programmes avec accès à un enseignement supérieur avec une orientation plus professionnelle) ; et

- Les programmes C, sans accès direct à l'enseignement supérieur (c'est-à-dire les programmes terminaux ou donnant uniquement accès à d'autres programmes des niveaux 3 ou 4 de la CITE 1997).

La CITE 2011 classe les programmes selon le type d'achèvement du niveau et l'accès à d'autres programmes et niveaux. Les destinations A et B de la CITE 1997 ont été combinées en une seule catégorie dans la CITE 2011 : achèvement (complet) du niveau 3 de la CITE 2011 avec accès direct à l'enseignement supérieur. La destination C de la CITE 1997 se subdivise en trois catégories dans la CITE 2011 :

- achèvement (complet) du niveau 3 de la CITE 2011 sans accès direct à l'enseignement supérieur ;

- achèvement (partiel) du niveau 3 de la CITE 2011 sans accès direct à l'enseignement post-secondaire ; et

- insuffisant pour un achèvement complet ou partiel du niveau 3 de la CITE 2011.

\section{DIRECTIVES POUR LA CLASSIFICATION DES PROGRAMMES ET DE LEURS CERTIFICATIONS AU NIVEAU 3 DE LA CITE}

Les programmes du deuxième cycle de l'enseignement secondaire sont plus spécialisés que ceux du premier cycle de l'enseignement secondaire. Ils proposent un éventail plus large d'options et de filières pour achever l'enseignement secondaire (\$163). L'éventail de matières étudiées par un élève est en général moins large que dans les niveaux d'éducation inférieurs, mais le contenu est plus complexe et l'étude plus approfondie.

Les programmes proposés sont différenciés par orientation (\$167) et souvent par larges groupes de matières.

Les programmes généraux sont habituellement conçus pour les élèves prévoyant de progresser vers des études académiques ou professionnelles dans l'enseignement supérieur. Les élèves commenceront souvent par se spécialiser dans des domaines spécifiques, tels que les sciences, les sciences humaines ou sociales, même s'ils doivent continuer à suivre des cours dans des matières fondamentales comme la langue nationale, les mathématiques et éventuellement une langue étrangère. L'achèvement complet de ces programmes est presque toujours le critère d'admission pour les premiers diplômes au niveau universitaire ou équivalent (Exemples Groupe 1). II peut également exister des programmes généraux au niveau 3 de la CITE ne donnant pas accès à l'enseignement supérieur, mais ils sont plutôt rares comparativement. Un exemple en est toutefois inclus dans les Exemples Groupe 10.

Dans certains pays, les critères d'admission pour les programmes du niveau 6 de la CITE sont différents de ceux du niveau 5 de la CITE. Quand c'est le cas, les deux certifications doivent être classées dans le niveau 3 de la CITE.

Les programmes professionnels existent aussi bien pour proposer des options à des jeunes qui sinon quitteraient l'école sans certification d'un programme du deuxième cycle du secondaire (Exemples Groupe 2) que pour ceux qui souhaitent se préparer à des emplois de niveau ouvrier qualifié et/ou technicien (Exemples Groupe 3).

Les programmes de seconde chance ou de réintégration conçus pour réviser les matières déjà abordées dans les programmes du deuxième cycle du secondaire (Exemples Groupe 4) ou offrir des opportunités à des jeunes de changer de filière ou d'accéder à une profession nécessitant une certification de fin de deuxième cycle du secondaire à laquelle leurs études en cours ne leur donne pas droit (Exemples Groupe 5) sont classés dans le niveau 3 de la CITE (§179).

Par contre, les programmes postérieurs à l'achèvement complet du deuxième cycle de l'enseignement secondaire qui sont soit destinés à élargir les options de progression vers l'enseignement supérieur, soit des programmes professionnels de second cycle doivent être classés au niveau 4 de la CITE (\$185). Les programmes professionnels de second cycle qui se déroulent en parallèle à d'autres programmes au second cycle de l'enseignement secondaire (pour la plupart des programmes généraux) doivent normalement être classés dans le niveau 3 de la CITE (§179).

Le deuxième cycle de l'enseignement secondaire dure entre deux et cinq ans, trois ans étant la durée la plus fréquente. Les programmes de ce niveau peuvent être plus courts dans certains pays où il existe une succession de programmes 
dans le niveau, chacun menant à une certification différente reconnue nationalement. Des programmes séquentiels du second cycle de l'enseignement secondaire peuvent exister quand la fin de la scolarité obligatoire se produit au cours du deuxième cycle de l'enseignement secondaire ou quand il y a des points de sortie communs dans le niveau qui doivent être documentés par une certification reconnue (par exemple, pour rechercher un emploi ou poursuivre sa formation). Quand une telle succession existe, il s'agit le plus souvent d'une succession de programmes généraux bien qu'une succession professionnelle soit également possible (Exemples Groupe 7).

\section{Programmes couvrant le deuxième cycle de l'enseignement secondaire et un autre niveau de la CITE}

II faut être particulièrement attentif aux programmes couvrant le deuxième cycle de l'enseignement secondaire et les niveaux précédents ou postérieurs. Dans ces cas, seuls les années d'études, phases ou cycles correspondant aux critères des niveaux respectifs (niveau 2, 3, 4 ou 5 de la CITE) doivent être classés dans ces niveaux. (§171)

(§173) Si la durée théorique d’un programme professionnel du niveau 3 de la CITE est de deux années plus longue (ou davantage) que la durée théorique d'un programme général du niveau 3 de la CITE dans le même système éducatif, il faut considérer que le programme couvre le deuxième cycle de l'enseignement secondaire (niveau 3 de la CITE) et l'enseignement post-secondaire non-supérieur (niveau 4 de la CITE) ou l'enseignement supérieur de cycle court (niveau 5 de la CITE). Les années d'études, phases ou cycles qui se prolongent audelà du programme général du niveau 3 de la CITE doivent être classés au niveau 4 ou 5 de la CITE selon la complexité de leur contenu. (Exemples Groupe 6).

\section{NIVEAU D'ÉDUCATION ATTEINT AU NIVEAU 3 DE LA CITE}

En vue de mesurer le niveau d'éducation atteint, la CITE 2011 opère une distinction entre achèvement complet et partiel du deuxième cycle de l'enseignement secondaire.

L'achèvement partiel d'un niveau a une signification très spécifique et n'est pas synonyme d' "études incomplètes » ou d' "études limitées » à ce niveau. Un programme menant à un 'achèvement partiel d'un niveau' est un programme donnant lieu à une certification reconnue par les autorités nationales compétentes pour l'éducation décernée aux élèves ayant achevé le programme avec succès à un point spécifique dans la succession de programmes mais qui n'est pas équivalente à la certification décernée lors de l'achèvement complet de l'ensemble de la succession de programmes. Ce programme appartient à une succession de programmes dans ce niveau de la CITE sans toutefois constituer le dernier programme de cette succession à ce niveau. Le programme doit également représenter au moins onze années en durée cumulée d'enseignement depuis le début de l'enseignement primaire et au moins deux années d'enseignement (cumulé) dans le deuxième cycle de l'enseignement secondaire (Exemples Groupe 7). Veuillez noter que l'achèvement partiel d'un niveau est également différent de la sous-catégorie 'non achèvement du niveau'. Cette dernière fait référence à des programmes ne satisfaisant pas les exigences de durée pour un achèvement partiel ou complet de niveau et menant donc à un niveau d'éducation atteint inférieur à celui du programme (voir Exemples Groupe 9).

(§182) En ce qui concerne le niveau d'éducation atteint, les certifications reconnues des programmes du niveau 3 de la CITE considérés comme insuffisants pour être pris en compte comme un achèvement [ou un achèvement partiel] du niveau 3 de la CITE sont classées dans le niveau 2 de la CITE.

(§183) De même, les certifications reconnues des programmes du niveau 4 de la CITE considérés comme insuffisants pour être pris en compte comme achèvement du niveau 4 de la CITE sont classées dans le niveau 3 de la CITE.

Si le programme ne mène pas à des certifications reconnues (ou si l'achèvement complet n'est pas reconnu formellement d'une autre façon par les autorités nationales compétentes), le concept d'achèvement partiel d'un niveau ne s'applique pas. Dans ce cas, le niveau d'éducation atteint de niveau CITE 3 peut être accordé seulement à la fin de la séquence de programmes, quand la certification correspondante est obtenue 
Les programmes du niveau 3 de la CITE ne donnant pas un accès direct à un niveau supérieur de la CITE et représentant un nombre d'années d'études moindre soit depuis le début de l'enseignement primaire (c'est-à-dire moins de onze ans) ou dans le deuxième cycle de l'enseignement secondaire (c'est-à-dire moins de deux ans) ou les deux sont considérés comme insuffisants pour un achèvement partiel ou complet du deuxième cycle de l'enseignement secondaire, et donnent un niveau d'éducation atteint correspondant au premier cycle de l'enseignement secondaire (Exemples Groupe 8).

La CITE subdivise les programmes professionnels menant à un achèvement complet du deuxième cycle de l'enseignement secondaire entre ceux donnant accès à l'enseignement supérieur (Exemples Groupe 9) et ceux ne le donnant pas (Exemples Groupe 10). Les deux sont toutefois considérés comme des programmes de niveau d'éducation atteint de niveau CITE 3. Ce dernier groupe de programmes peut donner accès à l'enseignement post-secondaire non-supérieur ou non.

Dans certains cas, un programme peut mener à plusieurs certifications différentes. Le programme doit être classé en fonction des caractéristiques de la certification donnant l'accès le plus large à des études ultérieures. Le niveau d'éducation atteint par les personnes ayant achevé un tel programme doit être déterminé en fonction de la certification la plus élevée décernée à ces personnes (Exemples Groupe 11).

Les utilisateurs de la classification souhaitant répartir les résultats selon un ensemble de catégories de niveau d'éducation atteint plus simplifié que cela n'est acceptable dans la CITE-A (c'est-à-dire un niveau de la CITE à 1 chiffre) peuvent se voir dans l'obligation de décider comment traiter l'achèvement partiel d'un niveau. Selon le principal objectif des analyses à effectuer, deux approches différentes sont possibles :

(i) Certains utilisateurs voudraient savoir quel pourcentage de la population concernée a achevé chaque niveau d'enseignement de la CITE avec succès (dans le cas des programmes séquentiels, l'achèvement du dernier programme dans la succession des programmes du niveau de la CITE). Pour cela, ils doivent classer l'achèvement partiel d'un niveau au niveau inférieur au niveau d'études (c'est-à-dire au niveau premier cycle du secondaire dans le cas de l'achèvement partiel du deuxième cycle de l'enseignement secondaire).

(ii) D'autres utilisateurs voudraient mesurer au moins certaines études réussies et reconnues à un niveau donné notamment lorsque cela peut représenter un point de sortie potentiel du système éducatif ou la première obtention d'un diplôme dans un niveau de la CITE donné. Pour cela, ils doivent classer l'achèvement partiel d'un niveau au niveau CITE du niveau d'études (c'est-à-dire au niveau du deuxième cycle de secondaire en cas d'achèvement partiel du deuxième cycle de l'enseignement secondaire).

Les deux approches sont valables. II incombe à l'utilisateur/l'analyste de décider de l'approche la plus appropriée en fonction des circonstances. II est recommandé que le traitement de l'achèvement partiel utilisé pour le calcul des indicateurs sur le niveau d'éducation atteint soit indiqué par des métadonnées accompagnant ces statistiques.

\section{EXEMPLES DE PROGRAMMES ET DE CERTIFICATIONS CLASSÉS AU NIVEAU 3 DE LA CITE}

(§165) Les programmes destinés à être classés au niveau 3 de la CITE peuvent être désignés de plusieurs manières : école secondaire ou lycée (cycle 2/ dernières années d'études de l'enseignement secondaire). En vue de la comparabilité internationale, l'expression « deuxième cycle de l'enseignement secondaire » est utilisée afin de qualifier le niveau 3 de la CITE.

\section{Exemples Groupe 1 :}

Programmes généraux donnant accès à des programmes de premier diplôme de l'enseignement supérieur (niveaux 6 ou 7 de la CITE 2011) [CITE-P : 344 ; CITE-A : 344]

Égypte - (Secondaire général). Ce programme de l'enseignement secondaire général exige la possession du certificat d'éducation de base, décerné lors de l'achèvement complet de l'éducation de base (CITE 2). Le programme de l'enseignement secondaire général dure trois ans, au terme desquels les élèves reçoivent le certificat du secondaire général donnant accès à des niveaux d'enseignement plus élevés, parmi lesquels l'enseignement supérieur ou nonsupérieur. (CITE-P /CITE-A : 344) 
Estonie - Üllkeskharidus (Enseignement secondaire général). Il s'agit d'un programme de trois ans menant au certificat d'achèvement du deuxième cycle du secondaire général qui donne un accès direct à des programmes Bakalaureuseõpe (programmes de niveau licence). En général, les élèves débutent le programme à 16 ans, après avoir achevé le premier cycle de l'enseignement secondaire avec succès. (CITE-P/CITE-A : 344)

France - Enseignement de second cycle général du second degré conduisant au baccalauréat général ou technologique ou au brevet de technicien. II s'agit d'un programme de trois ans qui suit l'achèvement complet du premier cycle de l'enseignement secondaire. II constitue le parcours typique vers l'enseignement supérieur et quasiment tous les détenteurs de ces certifications poursuivent leurs études dans de tels programmes. (CITE-P/CITE-A : 344)

Allemagne - Allgemeinbildende Programme im Sekundarbereich II (Écoles du deuxième cycle du secondaire, général). Ce sont des programmes du deuxième cycle du secondaire général qui durent trois ans (Gymnasiale Oberstufe), comprennent les Années d'études 10 à 12 ou 11 à 13, et mènent à la Hochschulreife (certification d'entrée à l'université). Ces programmes du Gymnasium (y compris Berufliches Gymnasium), Integrierte Gesamtschule ou Freie Waldorfschule (lycées, collèges ou écoles Waldorf) sont fréquentés par des élèves qui ont obtenu le Mittlerer Schulabschluss (certificat du premier cycle du secondaire avec accès au deuxième cycle du secondaire). Les diplômés de ces programmes ont le droit d'accéder aux programmes de niveau 6 ou 7 de la CITE. (CITE-P/CITE-A : 344)

Arabie saoudite - (Enseignement secondaire général). II s'agit d'un programme éducatif général à temps plein de trois ans nécessitant l'achèvement de l'enseignement intermédiaire (CITE 2). Lors de l'achèvement du programme, les élèves se voient décerner le certificat national de secondaire général, qui mène à l'enseignement supérieur. (CITE-P / CITE-A : 344)

Swaziland - Senior Secondary Certificate (Certificat du deuxième cycle de l'enseignement secondaire). II s'agit d'un programme de deuxième cycle du secondaire de deux ans proposés aux diplômés du premier cycle du secondaire donnant un accès direct à tous les programmes d'enseignement post-secondaire. L'âge officiel d'entrée est 16 ans. (CITE-P/CITE-A : 344)

Suisse - Gymnasiale Maturität, maturité gymnasiale, maturità (études menant à un certificat de fin d'études). Ce sont des programmes généraux de 3,5 ans destinés aux jeunes ayant achevé le premier cycle de l'enseignement secondaire avec succès et souhaitant poursuivre leurs études au niveau universitaire. Ils les préparent à l'examen d'entrée à l'université. (CITE-P/CITE-A : 344)

\section{Exemples Groupe 2 :}

Programmes professionnels destinés aux jeunes souhaitant se préparer à une entrée directe sur le marché du travail ou qui sinon quitteraient l'école sans certification d'un programme du deuxième cycle du secondaire [CITE-P : 351 ou 353 ; CITE-A : 254 ou 353]

Bulgarie - Profesionalni programi - parva stepen na profesionalna kvalifikatsiya sled 8 klas (programmes techniques et professionnels après achèvement de la 8 e année menant à une certification professionnelle de premier niveau). Ce sont des programmes professionnels de deux ans qui débutent après l'achèvement du premier cycle de l'enseignement secondaire et mènent au Profesionalni programi- parva stepen na profesionalna kvalifikatsiya (Certification professionnelle de premier niveau). Les programmes ne mènent pas à un certificat de fin du deuxième cycle du secondaire - Sredno obrazovanie - donnant un accès direct à l'enseignement supérieur. Comme le programme ne représente que dix ans d'études depuis le début de l'enseignement primaire, il est insuffisant pour un achèvement complet ou partiel du niveau 3 de la CITE. (CITE-P : 351 ; CITE-A : 254)

Slovénie - Nižje poklicno izobraževanje (Deuxième cycle de l'enseignement secondaire professionnel de courte durée). II s'agit d'un programme professionnel de deux à trois ans destiné aux jeunes ayant achevé neuf années d'éducation de base (obligatoire) avec succès ou ayant achevé un programme éducatif adapté pour les enfants ayant des besoins éducatifs spéciaux. Le programme est également accessible aux élèves ayant fréquenté l'éducation de base pendant neuf ans mais ne l'ayant pas achevé avec succès (ce qui signifie qu'ils ont rempli l' " obligation d'éducation de base "). Conformément à la loi, ces programmes durent deux à trois ans et se terminent par un examen final. lls renforcent les connaissances générales acquises par les élèves dans les dernières années d'éducation de base et enseignent de nouvelles connaissances et certifications générales et professionnelles pour des emplois simples. (CITE-P/CITE-A : 353) 


\section{Exemples Groupe 3 : \\ Programmes professionnels préparant à des emplois de niveau ouvrier qualifié ou technicien [CITE-P : 353 ou 354 ; CITE-A : 353 ou 354]}

Australie - Certificat III. Ce sont des cours professionnels avancés pour des métiers reconnus, des techniciens et d'autres professions qualifiées. II n'y a pas d'exigences minimales formelles d'entrée mais les jeunes qui accèdent à ce programme ont généralement 15 ou 16 ans. Ces programmes ont généralement une durée de 1 à 2 ans au niveau 3 de la CITE ou une durée cumulée de 14 à 15 ans depuis le début du niveau 1 de la CITE. Les élèves qui l'achèvent avec succès n'ont pas accès à l'enseignement supérieur mais peuvent accéder à des programmes de Certificat IV au niveau 4 de la CITE 2011 ou entrer sur le marché du travail. (CITE-P/CITE-A : 353)

France - Enseignement de second cycle professionnel des écoles sociales conduisant aux diplômes de moniteurs éducateurs et équivalents. Ce sont des programmes professionnels de deux ans préparant à certains services sociaux (par ex. moniteur éducateur, technicien de l'intervention sociale et familiale) destinés à des élèves plus âgés ayant achevé le premier cycle de l'enseignement secondaire avec succès. Les certifications sur lesquelles ils débouchent sont équivalentes au baccalauréat et donnent accès à des programmes de l'enseignement supérieur. (CITE-P/CITE-A : 354)

France - Enseignement de second cycle professionnel du second degré conduisant au Baccalauréat Professionnel ou équivalent. Ce programme de trois ans se déroule principalement dans un établissement d'enseignement/de formation mais inclut également des périodes de formation en entreprise et prépare les participants à entrer dans la vie professionnelle. II est également possible d'obtenir le Bac professionnel par l'apprentissage, le temps d'instruction étant réparti entre un établissement d'enseignement/de formation et une entreprise. La certification donne un accès direct au marché du travail. Une minorité de diplômés poursuit ses études à un niveau plus élevé, généralement pour obtenir un Brevet de technicien supérieur (BTS) au niveau 5 de la CITE. (CITE-P/CITE-A : 354)

Allemagne - Berufsschulen (Duales System) Erstausbildung (Écoles professionnelles (Système de double) Formation). C'est une forme d'apprentissage spéciale de 2, 3 ou 3,5 ans combinant enseignement et formation, à la fois dans une école professionnelle et une entreprise. Dans la majorité des cas, elle dure trois ans. Les élèves doivent avoir achevé le niveau 2 de la CITE. Le programme mène à une Lehrabschluss (Certification du double système). Les diplômés sont qualifiés pour accéder aux Fachoberschulen (un an), aux Fachschulen ou au marché du travail. (CITE-P/CITE-A : 354)

Liban - (secondaire professionnel). Ce programme exige l'achèvement de la CITE 2 (général ou professionnel) et il dure trois ans à plein temps. Normalement, les participants ont entre 15 et 17 ans. Ce programme professionnel de niveau secondaire considéré comme un achèvement complet du niveau 3 de la CITE 2011 ne donne pas un accès direct à un niveau plus élevé de la CITE. (CITE-P/CITE-A : 353)

République slovaque ${ }^{2}$ - Stredá odborná škola - štúdium s maturity (École secondaire spécialisée - menant à la maturité). II s'agit d'un programme de quatre ans offrant à la fois un enseignement général et une formation professionnelle axée sur l'exécution d'activités professionnelles plus exigeantes. Les élèves qui l'achèvent avec succès reçoivent à la fois une certification générale - Vysvedčenie o maturitnej skúške (maturita) - et une certification professionnelle - Výučný list (certificat d'apprentissage) - et peuvent poursuivre leur parcours soit dans l'enseignement supérieur, soit sur le marché du travail. (CITE-P/CITE-A : 354)

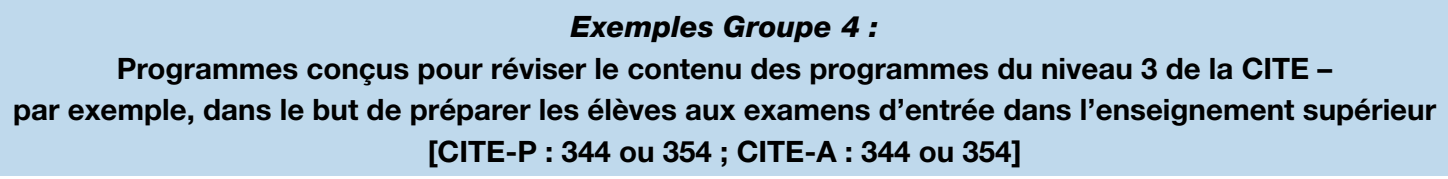

Australie - Enabling courses. Ce sont des programmes de courte durée (moins d'un an) complémentaires ou constituant une passerelle offerts par des universités à des élèves désavantagés ayant besoin d'une préparation supplémentaire avant de débuter un cours formel ou à des élèves universitaires pour renforcer leurs acquis dans un domaine d'étude particulier. Les programmes sont destinés à renforcer des études déjà entreprises dans le second cycle de l'enseignement secondaire afin d'assurer que les participants sont fin prêts pour débuter l'enseignement supérieur. (CITE-P/CITE-A : 344) 


\section{Exemples Groupe 5 :}

Programmes de seconde chance ou de réintégration pour les élèves n'ayant pas encore achevé le deuxième cycle de l'enseignement secondaire [CITE-P : 343, 344, 353 ou 354 ; CITE-A : 343, 344, 353 ou 354]

Maroc - (Formation professionnelle : niveau technicien). Ce programme professionnel de deux ans est proposé aux élèves ayant atteint la dernière année du deuxième cycle de l'enseignement secondaire général. II donne une seconde chance à ceux qui veulent changer pour une filière professionnelle. Au terme du programme, les élèves se voient décerner un diplôme technique qui donne uniquement un accès direct au marché du travail. (CITE-P/CITE-A : 353)

Pays-Bas - VAVO-HAVO et VAVO-WWO (deuxième cycle de l'enseignement secondaire général pour adultes). Ce sont des programmes à temps partiel d'un à trois ans menant aux certifications hoger algemeen voortgezet onderwijs (HAVO) et voorbereidend wetenschappelijk onderwijs (VWO) donnant un accès direct à l'enseignement supérieur. II s'agit de programmes de seconde chance et ils sont les équivalents à temps partiel des programmes initiaux à temps plein de deux ans pour le HAVO et de trois ans pour le WWO. Le critère d'admission est l'achèvement du premier cycle de l'enseignement secondaire. Les programmes pour adultes mènent aux mêmes certifications que les programmes initiaux mais avec un âge d'entrée un peu plus élevé, qui est généralement 18 ans au lieu de 16 ans. (CITE-P/CITE-A : 344)

Slovénie - Poklicni tecaj in poklicna matura (Cours professionnel menant à une Maturité professionnelle). II s'agit d'un cours professionnel d'un an constituant une passerelle entre l'enseignement général et professionnel. II permet aux diplômés des établissements généraux, classiques et autres (ou ceux ayant achevé la dernière année de ces programmes sans avoir passé l'examen général de maturité) d'obtenir une première certification professionnelle. Généralement, les cours professionnels menant à l'examen de maturité professionnelle donnent droit aux mêmes certifications que les programmes techniques de quatre ans du deuxième cycle du secondaire donnant accès à l'enseignement supérieur et au marché du travail. (CITE-P/CITE-A : 354)

\section{Exemples Groupe 6:}

Programmes couvrant le niveau 3 et d'autres niveaux de la CITE

Autriche - Berufsbildende höhere Schule (École supérieure technique et professionnelle). Programme professionnel de cinq ans couvrant le niveau 3 de la CITE (années 1 à 3) et le niveau 5 de la CITE (années 4 à 5). Le critère d'admission de ce programme est le premier cycle de l'enseignement secondaire. L'achèvement complet de la troisième année est équivalent à une certification professionnelle du secondaire (niveau 3 de la CITE) et la certification finale (après cinq ans) est directement équivalente à d'autres certifications classées dans le niveau 5 de la CITE. II permet aussi d'obtenir des crédits pour un transfert dans des programmes des niveaux 6 ou 7 e la CITE dans des domaines apparentés. Le programme donne aux élèves le droit d'exercer une profession de haut niveau dans les domaines de l'ingénierie, du commerce, des arts et de l'artisanat, du tourisme, du commerce, des services domestiques, de l'enseignement dans les jardins d'enfants et autres. (Années 1 à 3 : CITE-P/CITE-A : 354 ; Années 4 à 5 : CITE-P : 554 ; CITE-A : 550)

Kazakhstan - Техническое и профессиональное образование по подготовке кадров по массовым профессиям технического и обслужсиваюегг труда (Formation technique et professionnelle dans des professions techniques). II s'agit de programmes de trois à quatre ans qui suivent l'achèvement du premier cycle de l'enseignement secondaire. Ils sont destinés à former des ouvriers qualifiés et à leur décerner le certificat de fin de deuxième cycle de l'enseignement secondaire (аттестат об общем среднем образовании) après les deux premières années et un diplôme technique et professionnel avec une certification technique (диплом о техническом и профессиональном образовании с присвоением технической квалификаиии) après l'achèvement de la troisième ou de la quatrième année. Ces programmes couvrent les niveaux 3 et 4 de la CITE, les deux premières années étant classées dans le niveau 3 de la CITE et la troisième et la quatrième dans le niveau 4 de la CITE. (Années 1 à 2 : CITE-P/CITE-A : 353 ; Années 3 à 4 : CITE-P/CITE-A : 454)

Kazakhstan - Техническое и профессиональное образование по подготовке спечиалистов среднего звена и предусматривающие овладение сложными (смежными) профессиями и практическими навыками выполнения работ во всех отраслях экономики, связанными с высокими технологиями и профессиональной деятельностью (Formation technique et professionnelle de cadres intermédiaires avec maîtrise des métiers et des compétences complexes impliquant un niveau élevé de technologie). II s'agit de programmes de quatre à cinq ans qui suivent l'achèvement du premier cycle de l'enseignement secondaire. Les programmes sont conçus pour enseigner aux participants les connaissances et les compétences professionnelles nécessaires pour des métiers exigeant un enseignement du niveau 
du supérieur. Ils décernent le certificat de fin de deuxième cycle de l'enseignement secondaire (ammестат об общем среднем образовании) après les deux premières années et un diplôme technique et professionnel avec une certification technique (диплом о техническом и профессиональном образовании с присвоением технической квалификации) аргѐs l'achèvement de la quatrième ou de la cinquième année. Ces programmes couvrent les niveaux 3 et 5 de la CITE, les deux premières années étant classées dans le niveau 3 de la CITE et la troisième, la quatrième et la cinquième dans le niveau 5 de la CITE. (Années 1 à 2 : CITE-P/CITE-A : 354 ; Années 3 à 4/5 : CITE-P : 554 ; CITE-A : 550)

\section{Exemples Groupe 7 : \\ Programmes et certifications ayant pour résultat l'achèvement partiel d'un niveau [CITE-P : 342 ou 352 ; CITE-A : 342 ou 352]}

Ancienne République yougoslave de Macédoine ${ }^{3}$ - Strucno osposobuvanje (Formation professionnelle). II s'agit d'un programme de deux ans qui suit l'achèvement du premier cycle de l'enseignement secondaire. Les élèves qui l'achèvent avec succès se voient décerner un diplôme et peuvent poursuivre leurs études dans les programmes professionnels de trois ou quatre ans du deuxième cycle de l'enseignement secondaire (Sredno obrazovanie - redovno ou Sredno obrazovanie - redovno strucno). Ce dernier programme donne un accès direct à l'enseignement supérieur. Le programme de formation professionnelle de deux ans est destiné à donner accès à d'autres programmes du deuxième cycle de l'enseignement secondaire et appartient donc à une succession de programmes dans le niveau de la CITE. (CITE-P/CITE-A : 352)

Lituanie - Profesinio mokymo programa, skirta asmenims, turintiems pagrindinị išsilavinimą ir nesiekiantiems vidurinio išsilavinimo (Programmes d'enseignement professionnel pour les personnes ayant une éducation de base mais n'essayant pas d'obtenir le certificat de maturité - Brandos atestatas - au terme de l'enseignement secondaire). II s'agit de programmes de deux ans destinés aux élèves ayant achevé le premier cycle de l'enseignement secondaire avec succès et menant uniquement à une certification professionnelle (Profesinés kvalifikacijos pažyméjimas). Les élèves ayant achevé ce programme avec succès peuvent accéder au programme professionnel de trois ans (Profesinio mokymo programa, skirta asmenims, turintiems pagrindinị išsilavinimą ir siekiantiems vidurinio išsilavinimo) menant également au certificat de maturité (Brandos atestatas). Bien que les diplômés du programme professionnel de deux ans peuvent déjà entrer sur le marché du travail, le programme est destiné à donner accès à un autre programme qui vient compléter le deuxième cycle de l'enseignement secondaire. Le programme de deux ans peut donc être considéré comme faisant partie d'une succession de programmes dans le niveau de la CITE. (CITE-P/CITE-A : 352)

Malaisie - Menengah Atas (Deuxième cycle du secondaire). II s'agit d'un programme de deux ans du deuxième cycle du secondaire général qui suit l'achèvement complet du premier cycle de l'enseignement secondaire. À la fin de l'année 5 , la dernière année d'études de ce programme, les élèves passent l'examen du certificat d'enseignement malaisien (Sijil Pelajaran Malaysia (SPM)) menant au Pra Universiti, qui est un programme de deux ans du deuxième cycle du secondaire menant à l'enseignement supérieur. On passe le SPM après 11 ans d'enseignement depuis le début du niveau 1 de la CITE. (CITE-P/CITE-A : 342)

Royaume-Uni - General Certificate of Secondary Education (GCSE, Certificat général d'enseignement secondaire) II s'agit d'un programme général de deux ans qui suit l'achèvement du premier cycle de l'enseignement secondaire. Jusqu'à récemment, il marquait la fin de la scolarité obligatoire au Royaume-Uni et constituait dès lors le premier point de sortie important du système éducatif. Les élèves étudient plusieurs certificats généraux - généralement 8 à 10 - dans différentes matières qui sont chacune évaluées individuellement au travers de travaux et d'examens terminaux. Chaque matière est cotée de $A^{\star}$ (la plus élevée) à G (la plus basse) ou U (non spécifié) en cas d'échec. Le pays considère que la réussite de cinq matières ou plus avec les notes $A^{*}$ à $C$ représente un achèvement partiel réussi du deuxième cycle du secondaire. II s'agit là d'un critère d'admission minimal typique pour des études ultérieures dans le deuxième cycle de l'enseignement secondaire - professionnel ou général - donnant accès à l'enseignement supérieur et/ou des professions spécifiques sur le marché du travail. Le programme de Certificat général d'enseignement secondaire appartient à une succession dans le niveau de la CITE sans toutefois constituer le dernier programme de cette succession. II représente onze années d'enseignement depuis le début de l'enseignement primaire et deux années d'enseignement dans le deuxième cycle de l'enseignement secondaire. Cinq matières réussies ou plus avec des notes élevées couvrent une part raisonnable du programme d'études du deuxième cycle du secondaire et sont donc considérées comme une certification reconnue. La certification ne donne pas accès au post-secondaire non-supérieur ou à l'enseignement supérieur. (CITE-P/CITE-A : 342) 


\section{Exemples Groupe 8 :}

Programmes du niveau 3 de la CITE insuffisants pour un achèvement de niveau, avec donc un niveau d'éducation atteint correspondant au niveau 2 de la CITE [CITE-P : 341 ou 351 ; CITE-A : 244 ou 254]

Bangladesh - Certificat d'école secondaire professionnelle. II s'agit d'un programme professionnel de deux ans nécessitant l'achèvement du Junior Secondary (premier cycle du secondaire, CITE 2). À la fin du programme, proposé dans des écoles et collèges techniques publics et non gouvernementaux, les élèves reçoivent le Certificat d'école secondaire professionnelle. La durée cumulée depuis le début du niveau 1 de la CITE n'est que de dix ans. Le programme est donc insuffisant pour un achèvement complet ou partiel du niveau 3 de la CITE. (CITE-P : 351 ; CITE-A : 254)

Bulgarie - Profesionalno obuchenie za vazrastni - treta stepen PK (Formation professionnelle pour adultes - certification professionnelle de troisième niveau). Ce programme professionnel prévu pour les adultes a une durée de 1,5 ans. II est conçu pour mener à une entrée directe sur le marché du travail. (CITE-P : 351 ; CITE-A : 254)

Nouvelle-Zélande - Année 11 - Certificat national d'achèvement éducatif. II s'agit du premier de trois programmes généraux successifs d'un an qui forment le parcours général typique dans le deuxième cycle de l'enseignement secondaire. II constitue le premier point de sortie important du système éducatif. II appartient à une succession de programmes qui représentent onze années d'enseignement depuis le début de l'enseignement primaire mais seulement une année d'enseignement dans le deuxième cycle de l'enseignement secondaire. La certification ne donne pas accès au post-secondaire non-supérieur ou à l'enseignement supérieur. (CITE-P : 341 ; CITE-A : 244)

Zambie - Certificat d'examen de commerce niveau II. Ce programme professionnel est proposé aux apprentis ayant achevé le premier cycle de l'enseignement secondaire et ayant au moins un an d'expérience professionnelle ou aux apprentis détenant un certificat de l'enseignement primaire et ayant au moins trois ans d'expérience professionnelle. La durée du programme varie de six mois à un an, ce qui est insuffisant pour un achèvement du niveau 3 de la CITE. (CITE-P : 351 ; CITE-A : 254)

\section{Exemples Groupe 9 : \\ Programmes/certifications professionnels donnant accès à l'enseignement supérieur [CITE-P : 354 ; CITE-A : 354]}

Japon - Koutou senmon gakko (Collèges de Technologie, années 1-3). L'entrée dans les Koutou senmon gakko est possible après achèvement de la scolarité obligatoire. Ce programme a comme objectif de préparer des ingénieurs prometteurs et axés sur la pratique et d'étudier des matières générales au travers d'un programme de cours de cinq ans (3 premières années : (CITE-P : 354 ; quatrième et cinquième année : CITE-P : 554). La plupart des élèves ayant achevé les trois premières années de Koutou senmon gakko iront en quatrième année (CITE-P 554), mais ils bénéficieront aussi de la certification nécessaire pour entrer à l'université (CITE 6 ou 766). (Trois premières années de Koutou senmon gakko : CITE-P/CITE-A : 354)

Koweit - (Programme de diplôme d'infirmier). L'accès à ce programme professionnel exige au minimum l'achèvement complet de l'Année d'études 9, la dernière année d'études du premier cycle de l'enseignement secondaire. Bien que l'âge d'entrée théorique soit 15 ans, l'âge d'entrée le plus fréquent est 17 ans, en raison de multiples redoublements. Ce programme dure trois ans et est considéré comme un achèvement complet du niveau. Après avoir obtenu leur diplôme, les participants peuvent accéder à l'enseignement supérieur. (CITE-P : 354 ; CITE-A : 354)

Pays-Bas - WEB-middenkaderopleiding, voltijd bol en bb/ (Enseignement professionnel, formation de cadre intermédiaire (niveau 4) ; programmes éducatifs avec système double à temps plein dans un cadre scolaire). II s'agit d'un programme professionnel de trois ans qui suit l'achèvement complet d'un programme préprofessionnel de quatre ans du premier cycle de l'enseignement secondaire (Voorbereidend middelbaar beroepsonderwijs (VMBO) (beroepsgerichte, gemengde en theoretische leerwegen)). II existe également un programme à temps partiel menant à la même certification (MBO niv.4: middenkaderfunctionaris) mais les participants sont généralement plus âgés que dans le programme à temps plein. (CITE-P/CITE-A : 354) 


\section{Exemples Groupe 10: \\ Programmes/certification menant à un achèvement (complet) du deuxième cycle de l'enseignement secondaire mais ne donnant pas accès à l'enseignement supérieur [CITE-P : 343 ou 353 ; CITE-A : 343 ou 353]}

Afghanistan - Talimate Takhniki wa Maslaki (Enseignement technique et professionnel (Années d'études 10 à 14)). La durée théorique de ce programme professionnel est cinq ans après l'achèvement complet de l'Année d'études 9 (CITE 2). Au terme du programme, les élèves se voient décerner le certificat Shahadat Naama qui donne un accès direct au marché du travail et à des programmes de l'enseignement supérieur. Les trois premières années d'études du programme sont classées dans le deuxième cycle de l'enseignement secondaire. (Années d'études 10 à 12 : CITE-P/ CITE-A : 353 ; Années d'études 13 à 14 : CITE-P/CITE-A : 454)

Allemagne - Beamtenausbildung (mittlerer Dienst) (Formation pour fonctionnaires (Niveau intermédiaire)). Ce programme dure deux ans et forme de futurs fonctionnaires au niveau de cadre intermédiaire. II faut le certificat de fin d'études intermédiaires ou un équivalent reconnu pour être admis. Le programme peut être suivi dans des établissements d'administrations publiques à tous les niveaux régionaux (Bund, Länder, Regierungsbezirke, Kreise, Gemeinden) et dans des écoles spéciales pour l'administration publique. À l'achèvement du programme, les diplômés sont employés dans la fonction publique. Le programme ne donne pas accès à l'enseignement supérieur. (CITE-P/CITE-A : 353)

Israël - Batey sefer taasiyatiim le hanihim, misrad ha-kalkala, tlat shnati (Apprentissage et écoles techniques, Ministère de l'Économie, enseignement de trois ans). II s'agit d'un programme professionnel de trois ans qui suit l'achèvement complet du premier cycle de l'enseignement secondaire. Les élèves qui l'achèvent avec succès peuvent poursuivre leurs études dans des programmes de courte durée (7 à 10 mois) préparant à l'ingénierie ou à des métiers similaires (Mechina kdam handasaim) mais la plupart entre directement sur le marché du travail. Un nombre restreint de diplômés poursuit ses études au niveau post-secondaire non-supérieur. (CITE-P/CITE-A : 353)

Seychelles - Programme de deuxième cycle de l'enseignement secondaire. II s'agit d'un programme de deux ans du deuxième cycle du secondaire général qui suit l'achèvement complet du premier cycle de l'enseignement secondaire. Lors de l'achèvement du programme, les élèves se voient décerner le Secondary Five National Examination Certificate ou IGCSE (Cambridge) ou le DELF Scolaire (Diplôme d'Études en Langue Française), qui donnent un accès direct à l'enseignement post-secondaire non-supérieur mais non à l'enseignement supérieur. (CITE-P/CITE-A : 343)

\section{Exemples Groupe 11: \\ Programmes menant à plus d'une certification (et à leurs niveaux d'éducation atteints) [Tous les codes CITE-P ou CITE-A au niveau 3 de la CITE]}

Pologne - Trzyletnie liceum ogólnokształcące (Deuxième cycle du secondaire général). Il s'agit d'un programme de trois ans qui suit l'achèvement complet du premier cycle de l'enseignement secondaire. II mène à un certificat de fin d'études (Świadectwo ukończenia liceum ogólnokształcącego (wykształcenie średnie)) décerné à tous les élèves achevant le programme avec succès. Les élèves qui souhaitent aller à l'université peuvent aussi passer un examen de maturité (Matura - świadectwo dojrzałości). II est impossible d'accéder à l'université sans certificat Matura. Le programme est classé comme CITE-P 344 car il s'agit d'un programme général destiné à donner accès à l'université (et une majorité des élèves vise l'obtention du Matura). Toutefois, le niveau d'éducation atteint par les élèves qui achèvent le programme avec succès dépend des certifications obtenues à la fin du programme. Le certificat de fin d'études n'étant pas suffisant pour une entrée dans l'enseignement supérieur, il est classé comme CITE-A 343 alors que le certificat Matura est codé comme CITE-A 344. (CITE-P : 344 ; CITE-A : 343 ou 344)

\section{Notes}

1. Les numéros de paragraphe constituent des références à la classification CITE 2011. Pour plus de détails, veuillez consulter le Guide du lecteur.

2. L'Institut de statistique de I'UNESCO (ISU) et l'Union européenne (UE) utilisent « Slovaquie » comme dénomination officielle pour la "République slovaque".

3. L'Institut de statistique de I'UNESCO (ISU) et I'Union européenne (UE) utilisent "L'ancienne République yougoslave de Macédoine » comme dénomination officielle pour l' "Ancienne République yougoslave de Macédoine ". 


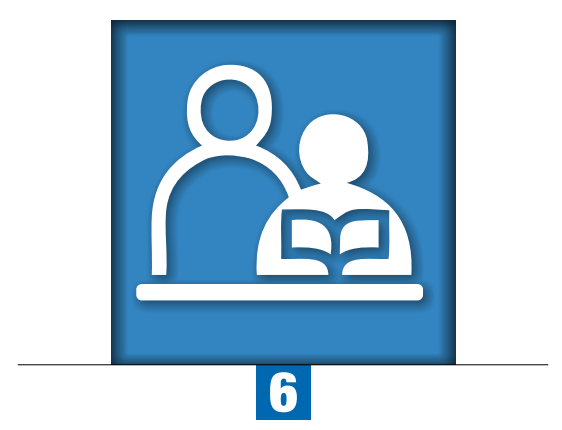

Chapitre 6

\section{Niveau 4 de la CITE 2011:}

\section{Enseignement post-secondaire non-supérieur}

Les programmes de l'enseignement post-secondaire non-supérieur ne sont pas beaucoup plus complexes que ceux du deuxième cycle de l'enseignement secondaire. Ils visent généralement à élargir plutôt qu'à approfondir les connaissances, aptitudes et compétences déjà acquises au travers de l'achèvement (complet) réussi du deuxième cycle de l'enseignement secondaire. Ils peuvent être destinés à accroître les options des participants sur le marché du travail ou à poursuivre ses études dans l'enseignement supérieur ou les deux.

La plupart des programmes du niveau 4 de la CITE ont une orientation professionnelle. Ils peuvent être désignés de plusieurs manières, par exemple : technician diploma, primary professional education ou préparation aux carrières administratives. En vue d'une comparabilité internationale, l'expression « enseignement post-secondaire non-supérieur » est utilisée pour qualifier le niveau 4 de la CITE. 


\section{DESCRIPTION}

\section{Définition}

$(\S 185)^{1}$ L'enseignement post-secondaire non-supérieur fournit des expériences d'apprentissage qui viennent compléter l'enseignement secondaire et préparent à l'entrée sur le marché du travail ainsi qu'à l'enseignement supérieur. II vise l'acquisition individuelle de connaissances, aptitudes et compétences dont le niveau de complexité est inférieur à celui de l'enseignement supérieur. Les programmes du niveau 4 de la CITE, ou "enseignement post-secondaire non-supérieur ", sont généralement conçus pour fournir aux individus qui ont achevé le niveau 3 de la CITE des certifications exigées pour accéder à l'enseignement supérieur ou à l'emploi lorsque leur certification de niveau 3 de la CITE ne leur donne pas cet accès. Par exemple, les diplômés des programmes d'enseignement général du niveau 3 de la CITE peuvent opter pour une certification professionnelle ne relevant pas de l'enseignement supérieur, alors que les diplômés de programmes d'enseignement professionnel du niveau 3 de la CITE peuvent décider d'améliorer leur niveau de certification ou de se spécialiser davantage. Le contenu des programmes du niveau 4 de la CITE ne sont pas suffisamment complexes pour être considérés comme des programmes de l'enseignement supérieur, même s'ils appartiennent clairement à l'enseignement post-secondaire.

(§187) Les programmes de ce niveau préparent habituellement à une entrée directe sur le marché du travail. Dans certains systèmes éducatifs, il existe des programmes d'enseignement général à ce niveau. Ces programmes ciblent principalement les élèves qui ont achevé le niveau 3 de la CITE et qui souhaitent améliorer leurs chances d'accéder à l'enseignement supérieur.

\section{Critères de classification (\$189)}

\section{Critères principaux}

a) Orientation

(§190) Les programmes du niveau 4 de la CITE ne sont pas considérés comme appartenant à l'enseignement supérieur. Il s'agit le plus souvent de programmes d'enseignement professionnel et terminaux qui préparent au marché du travail. Des programmes d'enseignement général peuvent exister à ce niveau dans certains systèmes éducatifs. Les programmes conçus pour réviser le contenu des programmes du niveau 3 de la CITE - par exemple, dans le but de préparer les élèves aux examens d'entrée dans l'enseignement supérieur - doivent être inclus dans le niveau 3 de la CITE.

b) Complexité du contenu supérieure à celle du niveau 3 de la CITE et inférieure à celle de l'enseignement supérieur

(§191) Les programmes du niveau 4 de la CITE servent souvent à élargir plutôt qu'à approfondir les connaissances, aptitudes et compétences des participants qui ont terminé un programme du niveau 3 de la CITE. Souvent, ces programmes ne sont pas d'un niveau significativement plus avancé que les programmes du niveau 3 de la CITE, mais leur contenu est généralement plus spécialisé ou plus détaillé que celui correspondant au deuxième cycle de l'enseignement secondaire. Les programmes sont clairement moins avancés que ceux de l'enseignement supérieur et ils peuvent se donner dans une variété d'établissements, pas uniquement dans les établissements considérés comme appartenant à l'enseignement post-secondaire non-supérieur.

c) Critères d'admission

(§186) L'achèvement complet d'un programme du niveau 3 de la CITE est exigé pour l'admission dans les programmes du niveau 4 de la CITE. Toutefois, les critères d'admission peuvent être moins exigeants que ceux requis pour les programmes de l'enseignement supérieur aux niveaux 5, 6 ou 7 de la CITE.

\section{Critères subsidiaires}

Aucun. 


\section{Dimensions complémentaires}

(§193) Deux dimensions permettent de distinguer les programmes éducatifs du niveau 4 de la CITE.

(§194) Orientation du programme :

- Général ; et

- Professionnel

(\$195) Achèvement de niveau et accès aux niveaux supérieurs de la CITE ;

- Non achèvement du niveau 4 de la CITE : modules ou cycles de programmes trop courts pour un achèvement de niveau. Ils ne donnent pas accès aux premiers programmes de l'enseignement supérieur aux niveaux 5,6 ou 7 de la CITE. L'achèvement complet de ces modules ou cycles n'est pas considéré comme un achèvement du niveau 4 de la CITE.

- Achèvement du niveau 4 de la CITE sans accès direct aux premiers programmes de l'enseignement supérieur aux niveaux 5, 6 ou 7 de la CITE : programmes conçus principalement pour une entrée directe sur le marché du travail.

- Achèvement du niveau 4 de la CITE avec accès direct aux premiers programmes de l'enseignement supérieur aux niveaux 5,6 ou 7 de la CITE : programmes conçus principalement pour élargir l'accès à l'enseignement supérieur.

(§198) En ce qui concerne le niveau d'éducation atteint, les certifications intermédiaires reconnues obtenues lors de l'achèvement complet d'un cycle d'un programme du niveau 5 de la CITE considéré comme insuffisant pour être pris en compte comme achèvement du niveau 5 de la CITE sont classées dans le niveau 4 de la CITE.

\section{CORRESPONDANCES ENTRE LA CITE 2011 ET LA CITE 1997}

(§279) Le niveau 4 de la CITE 2011, enseignement post-secondaire non-supérieur, correspond en grande partie au niveau 4 de la CITE 1997. Néanmoins, les programmes menant à une certification équivalente à celle du deuxième cycle de l'enseignement secondaire général sont classés au niveau 3 de la CITE 2011, alors qu'ils étaient souvent classés au niveau 4 de la CITE 1997. De plus, la CITE 2011 peut être implémentée différemment de la CITE 1997 car les critères principaux et subsidiaires ont été clarifiés. Ces différences peuvent avoir un impact sur la série chronologique des données de certains pays.

Les dimensions complémentaires du niveau 4 de la CITE sont différentes dans la dernière version de la CITE.

\section{a) Orientation du programme}

La CITE 1997 définit trois types d'orientation : général, préprofessionnel et professionnel. La CITE 2011 ne propose que deux orientations au niveau 4 de la CITE : général et professionnel. Les programmes classés au préalable dans la catégorie préprofessionnelle (CITE 1997) ne permettent pas l'obtention de certifications pertinentes pour le marché du travail et de ce fait sont maintenant classés pour la plupart dans l'enseignement général.

b) Achèvement de niveau et programme de destination

La CITE 1997 classait les programmes en fonction des programmes et des niveaux auxquels ils devaient donner accès (le programme de destination). Au niveau 4 de la CITE, trois destinations étaient définies dans la CITE 1997 :

A = programmes préparant à une entrée dans l'enseignement supérieur (niveau 5 de la CITE 1997);

$\mathrm{B}=$ programmes ne donnant pas accès à l'enseignement supérieur (conçus principalement pour une entrée directe sur le marché du travail).

La CITE 2011 classe les programmes selon le type d'achèvement du niveau et l'accès à d'autres niveaux. La destination A de la CITE 1997 est directement équivalente à la sous-catégorie achèvement (complet) du niveau 4 de la CITE 2011 avec accès direct à l'enseignement supérieur de la CITE 2011. La destination B de la CITE 1997 se subdivise en deux sous-catégories dans la CITE 2011:

- achèvement (complet) du niveau 4 de la CITE 2011 sans accès direct à l'enseignement supérieur ; et

- insuffisant pour un achèvement du niveau 4 de la CITE 2011. 


\section{DIRECTIVES POUR LA CLASSIFICATION DES PROGRAMMES ET DE LEURS CERTIFICATIONS CORRESPONDANTES RECONNUES AU NIVEAU 4 DE LA CITE}

Les programmes de l'enseignement post-secondaire non-supérieur ne sont pas beaucoup plus complexes que ceux du deuxième cycle de l'enseignement secondaire. Ils visent généralement à élargir plutôt qu'à approfondir les connaissances, aptitudes et compétences déjà acquises au travers de l'achèvement (complet) réussi du deuxième cycle de l'enseignement secondaire. Ils peuvent être destinés à accroître les options des participants sur le marché du travail ou à poursuivre ses études dans l'enseignement supérieur ou les deux.

Les programmes destinés à mener à des certifications supplémentaires du deuxième cycle du secondaire ou à donner accès à des professions autres que celles étudiées dans le deuxième cycle de l'enseignement secondaire doivent être classés dans le niveau 4 de la CITE. Cela inclut les programmes professionnels de second cycle ayant pour résultat une durée cumulée totale d'études depuis le début du niveau 3 de la CITE qui est significativement plus longue que le programme du deuxième cycle du secondaire général donnant un accès direct aux premiers diplômes des niveaux 6 ou 7 de la CITE. Les programmes professionnels proposés en parallèle à d'autres programmes du deuxième cycle du secondaire doivent normalement être classés au niveau 3 de la CITE.

La plupart des programmes du niveau 4 de la CITE ont une orientation professionnelle et offrent aux élèves ayant achevé une formation de deuxième cycle de l'enseignement secondaire avec succès les connaissances, aptitudes et compétences requises pour une profession spécifique. Dans de nombreux cas, ces programmes professionnels d'enseignement postsecondaire non-supérieur sont destinés uniquement à mener au marché du travail, sans donner accès à l'enseignement supérieur. Ces programmes sont classés dans la CITE-P : 453 ; CITE-A : 453 (voir Exemples Groupe 1).

Certains systèmes éducatifs proposent également des programmes d'enseignement général post-secondaire nonsupérieur. Ces programmes ciblent principalement les élèves qui ont achevé le deuxième cycle de l'enseignement secondaire et qui souhaitent améliorer leurs chances d'accéder à l'enseignement supérieur (\$187). Les élèves peuvent avoir terminé un programme professionnel du deuxième cycle du secondaire et souhaiter obtenir une certification de l'enseignement secondaire général. Autre possibilité : ils peuvent avoir terminé un enseignement général du deuxième cycle du secondaire mais souhaiter élargir leurs connaissances dans des matières ou des domaines spécifiques afin d'améliorer leurs chances dans le processus de candidature à l'enseignement supérieur. Ces programmes sont classés dans la CITE-P : 444 ; CITE-A : 444 (voir Exemples Groupe 2).

Les programmes de seconde chance ou de réintégration destinés aux élèves n'ayant pas achevé le deuxième cycle de l'enseignement secondaire avec succès, à améliorer les notes des certifications déjà obtenues à la fin du deuxième cycle de l'enseignement secondaire ou à changer de filière - généralement du général au professionnel - pour accéder à des programmes ou à des professions du deuxième cycle de l'enseignement secondaire auxquels les certifications du deuxième cycle du secondaire déjà obtenues ne donnent pas accès doivent être classés dans le niveau 3 de la CITE. (Voir le chapitre sur le niveau 3 de la CITE pour des exemples.)

Certains programmes professionnels CITE 4 peuvent aussi être conçus pour donner accès à l'enseignement supérieur quand l'élève ne bénéficiait pas auparavant de cet accès, ou peuvent améliorer et élargir les opportunités d'études dans l'enseignement supérieur bien que la certification du deuxième cycle du secondaire de l'élève lui donnait déjà accès à certains programmes de l'enseignement supérieur. D'autres programmes CITE 4 peuvent donner accès au marché du travail et à l'enseignement supérieur. Pour autant que les autres critères de la CITE 4 soient également satisfaits, ces programmes peuvent tous être considérés comme un achèvement de la CITE 4 avec accès à l'enseignement supérieur (voir Exemples Groupe 3).

Parfois, il peut s'avérer difficile de savoir si un programme post-secondaire non-supérieur donne un premier accès à l'enseignement supérieur ; s'il améliore les chances d'accès quand l'élève a déjà un accès à l'enseignement supérieur ; ou si le programme n'est pas destiné à donner accès à l'enseignement supérieur même si la certification précédente requise pour accéder au programme donnait déjà accès à l'enseignement supérieur (voir Exemples Groupe 4). Voici ci-dessous une directive possible dans ces cas.

\begin{tabular}{l|l|l}
\hline $\begin{array}{l}\text { Accès d'un programme CITE 3 } \\
\text { requis pour entrer }\end{array}$ & Accès d'un programme CITE 4 & $\begin{array}{l}\text { Codification avec troisième chiffre } \\
\text { (ClTE-P et CITE-A) }\end{array}$ \\
\hline $\begin{array}{l}\text { Pas d'accès direct à l'enseignement } \\
\text { supérieur }\end{array}$ & $\begin{array}{l}\text { Donne un premier accès à } \\
\text { l'enseignement supérieur }\end{array}$ & $\begin{array}{l}444 / 454-\text { achèvement avec accès direct } \\
\text { à l'enseignement supérieur }\end{array}$ \\
\hline $\begin{array}{l}\text { Accès direct à l'enseignement } \\
\text { supérieur }\end{array}$ & $\begin{array}{l}\text { Accès direct plus large à } \\
\text { l'enseignement supérieur }\end{array}$ & $\begin{array}{l}444 / 454-\text { achèvement avec accès direct } \\
\text { à l'enseignement supérieur }\end{array}$ \\
\hline $\begin{array}{l}\text { Accès direct à l'enseignement } \\
\text { supérieur }\end{array}$ & $\begin{array}{l}\text { Pas d'accès ultérieur ou plus large } \\
\text { à l'enseignement supérieur }\end{array}$ & $\begin{array}{l}\text { CITE-P : 443/453 - achèvement sans accès } \\
\text { direct au CITE-A supérieur : 444/454 - car } \\
\text { l'élève a déjà accès à l'enseignement supérieur }\end{array}$ \\
\hline
\end{tabular}


Le critère d'admission aux programmes de CITE 4 est l'achèvement complet de la CITE 3. Cela signifie l'achèvement complet d'un programme du deuxième cycle de l'enseignement secondaire classé comme achèvement (complet) du deuxième cycle de l'enseignement secondaire (c'est-à-dire CITE-P/CITE-A : 343, 353, 344 ou 354). Les programmes qui succèdent à un programme CITE 3 mais ne mènent pas à un achèvement du niveau doivent être classés dans le niveau 3 de la CITE.

Les programmes du niveau 4 de la CITE sont souvent destinés à des participants ayant dépassé l'âge du deuxième cycle de l'enseignement secondaire. Les critères d'admission peuvent être des certifications d'enseignement et une période d'expérience professionnelle pertinente (voir Exemples Groupe 5).

Les programmes du niveau 4 de la CITE ont généralement une durée équivalent temps plein variant entre six mois et deux ans. Les programmes durant plus de deux ans peuvent être classés dans le niveau 4 de la CITE. Cela n'empêche pas qu'il faut évaluer le niveau de complexité des programmes beaucoup plus longs que deux ans. II faut être particulièrement attentif à la classification des programmes très longs dans le niveau 4 de la CITE. Dans ces cas, le principal critère de distinction entre l'enseignement post-secondaire non-supérieur (niveau 4 de la CITE) et l'enseignement supérieur de cycle court (niveau 5 de la CITE) est le degré de complexité du programme (voir Exemples Groupe 6).

Lorsque des programmes de différentes durées mènent à la même certification, ou à des certifications reconnues comme équivalentes, ils peuvent normalement être classés dans le même niveau de la CITE. Toutefois, comme le niveau 4 de la CITE n'est pas beaucoup plus avancé que certains programmes du niveau 3 de la CITE - notamment les programmes de second cycle ou ceux menant à d'autres certifications que celles déjà obtenues dans le deuxième cycle de l'enseignement secondaire - cela peut déboucher sur des certifications décernées à d'autres participants de certains programmes du deuxième cycle du secondaire. L'équivalence des certifications n'est pas une raison suffisante pour classer un programme dans le niveau 3 de la CITE. Par ailleurs, certaines certifications du niveau 4 de la CITE sont reconnues au niveau national comme étant « supérieures " à celles décernées dans le niveau 3 de la CITE. Lorsque c'est le cas, tous les programmes menant à ces certifications " supérieures » doivent être considérés soit comme des programmes couvrant les niveaux 3 et 4 de la CITE, soit comme des programmes du niveau 4 de la CITE.

Les programmes d'enseignement post-secondaire non-supérieur peuvent être désignés de plusieurs manières (voir $\S 188)$. Toutefois, il est important de ne pas classer le programme par nom ou par établissement mais selon le niveau de complexité du contenu, dans une perspective internationale. De même, il faut noter que les programmes du niveau 4 de la CITE peuvent être donnés au sein d'une variété d'établissements, pas uniquement dans les établissements considérés comme appartenant à l'enseignement post-secondaire non-supérieur (\$191, 52). Dès lors, le type de prestataire de service d'éducation ou l'établissement ne peut pas être utilisé comme le principal critère pour distinguer l'enseignement du niveau 4 de la CITE de l'enseignement supérieur ou même de l'enseignement secondaire (voir Exemples Groupe 7).

\section{Différence entre les niveaux 4 et 5 de la CITE}

Le principal critère de distinction entre l'enseignement post-secondaire non-supérieur et l'enseignement supérieur de cycle court est le degré de complexité du programme. L'accès aux programmes des deux niveaux exige l'achèvement complet du deuxième cycle de l'enseignement secondaire. Les programmes du niveau 4 de la CITE élargissent la connaissance des élèves dans un certain domaine. Néanmoins, ces programmes ne sont pas d'un niveau significativement plus avancé que les programmes du niveau 3 de la CITE. Le niveau 5 de la CITE est bien plus élevé que le niveau 3 de la CITE. Les programmes du niveau 5 de la CITE approfondissent les connaissances, aptitudes et compétences des participants dans un domaine donné.

Les certifications obtenues au niveau 5 de la CITE sont considérées comme supérieures à celles du niveau 4 de la CITE. Les programmes de l'enseignement supérieur de cycle court peuvent aussi permettre d'obtenir des crédits pour un transfert dans des programmes de premier diplôme universitaire des niveaux 6 ou 7 de la CITE. Lors de l'achèvement du niveau 5 de la CITE, les personnes peuvent bénéficier d'un accès direct à ces programmes de premier diplôme universitaire et être exemptées de certains cours ou modules à ces niveaux. Les programmes du niveau 4 de la ClTE mènent à des certifications qui ne sont que légèrement supérieures à celles du niveau 3 de la CITE, et dans certains cas, ils peuvent même déboucher sur les mêmes certifications. Ils ne permettent pas d'obtenir des crédits pour des études dans l'enseignement supérieur.

\section{NIVEAU D'ÉDUCATION ATTEINT AU NIVEAU 4 DE LA CITE}

Le niveau d'éducation atteint au niveau 4 de la CITE exige l'achèvement complet - menant à une certification reconnue d'un programme qui représente une durée cumulée minimale d'au moins 6 mois au niveau 4 de la CITE. 
Le niveau 4 de la CITE inclut des modules ou des cycles de programmes d'une durée inférieure à 6 mois et donc trop courts pour un achèvement de ce niveau. L'achèvement complet de ces modules ou cycles de programmes est considéré comme un niveau 3 de la CITE en termes de niveau d'éducation atteint.

De même, les certifications résultant de l'achèvement complet de programmes classés au niveau 5 de la CITE mais ne satisfaisant pas les critères pour un achèvement de niveau seront classées au niveau 4 de la CITE comme niveau d'éducation atteint, même si ce niveau n'existe pas dans le pays. (Voir Exemples Groupe 7 dans le chapitre sur le niveau 5 de la CITE)

\section{EXEMPLES DE PROGRAMMES ET DE CERTIFICATIONS CLASSÉS AU NIVEAU 4 DE LA CITE}

(§188) Les programmes classés au niveau 4 de la CITE peuvent être désignés de plusieurs manières, par exemple : technician diploma, primary professional education ou préparation aux carrières administratives. En vue d'une comparabilité internationale, l'expression « enseignement post-secondaire non-supérieur » est utilisée pour qualifier le niveau 4 de la CITE.

\section{Exemples Groupe 1 : \\ Programmes professionnels du niveau 4 de la CITE suffisants pour un achèvement de niveau, conçus pour donner accès au marché du travail (sans accès direct à l'enseignement supérieur) [CITE-P : 453 ; CITE-A : 453]}

Égypte - (Les deux dernières années du programme technique de cinq ans). Ce programme professionnel long exige l'achèvement du niveau éducatif préparatoire (CITE 2). Au terme du programme de cinq ans, les élèves se voient décerner un premier diplôme technique qui donne uniquement accès au marché du travail. Un diplôme similaire est également décerné à la suite de l'achèvement d'un autre programme professionnel post-secondaire de deux ans (déjà classé dans la CITE 4). Dans le cadre de la soumission internationale de données, ce long programme est considéré comme couvrant les niveaux 3 et 4 de la CITE. Les trois premières années d'études sont classées comme un achèvement complet du deuxième cycle du secondaire sans accès à l'enseignement supérieur (CITE-P/CITE-A : 353) alors que les deux dernières années sont considérées comme de l'enseignement post-secondaire non-supérieur sans accès à l'enseignement supérieur. (CITE-P/CITE-A : 453)

Irlande - Certificat avancé en Agriculture Teagasc. Ce programme de formation professionnelle peut durer de 1,5 à trois ans. Le critère d'admission minimal est le deuxième cycle de l'enseignement secondaire. L'achèvement complet du programme donne droit à un certificat avancé Teagasc (Autorité irlandaise du Développement agricole et alimentaire) en Agriculture, menant directement au marché du travail. (CITE-P/CITE-A : 453)

Espagne - Certificados de Profesionalidad de nivel 3 (Certificat professionnel de niveau 3 - certification professionnelle de troisième niveau). Ces programmes préparent au marché du travail. Le critère d'admission général de ces programmes est l'achèvement du deuxième cycle de l'enseignement secondaire. Les personnes capables de démontrer leur capacité à venir à bout de ces programmes ou celles possédant une certification Certificado de Profesionalidad de nivel 2 dans le même domaine d'étude peuvent également y accéder. Le programme dure entre six mois et un an (entre 350 et 1110 heures d'études et d'enseignement/formation supervisé). (CITE-P/CITE-A : 453)

\section{Exemples Groupe 2 :}

Programmes généraux du niveau 4 de la CITE suffisants pour un achèvement de niveau, conçus pour améliorer les chances d'accéder à l'enseignement supérieur [CITE-P : 444 ; CITE-A : 444]

Canada - Programmes de diplôme pré-universitaire général post-secondaire de courte durée. Les programmes ont une durée pouvant aller jusqu'à deux ans après l'achèvement du deuxième cycle de l'enseignement secondaire. Ils sont considérés comme des programmes qualifiants en préparation à des études universitaires du premier cycle. Après avoir achevé ces programmes de diplôme, les élèves peuvent poser leur candidature pour des programmes de niveau licence (soit le niveau 6 de la CITE). (CITE-P/CITE-A : 444)

Allemagne - Sekundarbereich II, Berufsoberschule (Écoles professionnelles du deuxième cycle du secondaire). Dans les Berufsoberschule, les connaissances, aptitudes et compétences acquises par les élèves au cours de leur enseignement 
professionnel initial ou de leur formation professionnelle initiale constituent les fondements d'un enseignement théorique général étendu et spécialisé approfondi permettant aux élèves de suivre un cours dans l'enseignement supérieur. Les cycles de formation de trois à quatre ans mènent à une double certification : une certification professionnelle (par exemple les professions d'assistant ou les certifications professionnelles dans un certain nombre de professions reconnues nécessitant une formation formelle) et une certification d'entrée dans l'enseignement supérieur. La Berufsoberschule offre deux ans d'enseignement à temps plein et mène au Fachgebundene Hochschulreife. Les élèves peuvent obtenir le Allgemeine Hochschulreife en prouvant leur aisance dans une seconde langue étrangère. (CITE-P/CITE-A : 444)

Israël - Mechina kdam akademit (Programme préparatoire pré-académique). Ce sont des programmes généraux d'un an destinés aux élèves ayant achevé des programmes du deuxième cycle de l'enseignement secondaire ne donnant pas accès à l'enseignement supérieur, en vue d'améliorer les chances d'accéder à l'enseignement supérieur. L'achèvement complet du programme donne droit à un certificat Mechina qui remplace le certificat de maturité pour être accepté dans l'enseignement supérieur. (CITE-P/CITE-A : 444)

Israël-Mechina kdam handasaim (Programmes préparatoires pour des ingénieurs axés sur la pratique). Ces programmes de 7 à 10 mois sont destinés aux élèves ayant achevé le deuxième cycle de l'enseignement secondaire et souhaitant poursuivre une formation universitaire dans l'ingénierie. L'achèvement complet du programme permet d'obtenir la certification Teudat Sium Mechinat Handesaim (achèvement de l'enseignement professionnel post-secondaire nonsupérieur), qui donne accès à des programmes de l'enseignement supérieur de cycle court en ingénierie (Mahat) offerts par le Ministère de l'Économie. (CITE-P/CITE-A : 444)

Oman - (Année de base). Ce programme post-secondaire général est proposé aux élèves ayant achevé le deuxième cycle de l'enseignement secondaire (avec accès à l'enseignement supérieur) et souhaitant étendre leurs options d'études au niveau supérieur. L'âge d'entrée typique dans ce programme d'un an est 19 ans. Lors de son achèvement, les diplômés reçoivent un certificat de base, qui donne également accès à l'enseignement supérieur. (CITE-P /CITE-A : 444)

Qatar - (Programme de base). Ce programme post-secondaire d'un an est proposé aux élèves ayant achevé le deuxième cycle de l'enseignement secondaire (avec accès à l'enseignement supérieur) et souhaitant étendre leurs options d'études au niveau supérieur. Le programme est proposé dans les universités et dure un an. Lors de son achèvement, les diplômés reçoivent un certificat d'achèvement qui donne également accès à l'enseignement supérieur. (CITE-P /CITE-A : 444)

Suisse - Passerellenlehrgang/passerelle/passerella (Cours préparatoire à l'université pour les détenteurs d'un baccalauréat professionnel). II s'agit de programmes généraux d'un an qui préparent les diplômés du deuxième cycle de l'enseignement secondaire professionnel à une admission dans les programmes d'étude de l'enseignement supérieur. Lors de l'achèvement complet du programme, les élèves obtiennent une attestation d'aptitude universitaire (Passerellenzeugnis/Certificat de passerelle/Certificado de passerella) donnant accès à des programmes de niveau licence. (CITE-P/CITE-A : 444)

\section{Exemples Groupe 3 :}

Programmes professionnels du niveau 4 de la CITE suffisants pour un achèvement de niveau, conçus pour donner accès à l'enseignement supérieur et au marché du travail avec des certifications professionnelles plus avancées que dans la CITE 3 [CITE-P : 454 ; CITE-A : 454]

Canada - Programmes de formation généraux, techniques ou professionnels. Généralement, les élèves débutent ces programmes de un à deux ans à 18 ans après l'achèvement du deuxième cycle de l'enseignement secondaire. Ces programmes à l'orientation professionnelle mènent à des attestations ou à des certificats pour des professions spécifiques. L'achèvement des programmes donne également accès à des programmes d'enseignement supérieur de cycle court. (CITE-P/CITE-A : 454)

Estonie - Kutseõpe keskariduse basil (Enseignement professionnel fondé sur l'enseignement secondaire). Ce sont des programmes professionnels destinés aux élèves ayant achevé le deuxième cycle de l'enseignement secondaire. L'âge d'entrée typique est 19 ans et la durée peut varier entre 1 et 2,5 ans. Après l'achèvement, les élèves reçoivent un Certificat de l'enseignement secondaire professionnel fondé sur l'enseignement secondaire. Le programme donne également accès à des programmes de licence (Bakalaureuseõpe). (CITE-P/CITE-A : 454) 
France - Préparation à l'entrée d'une formation sélective de niveau BTS (Divers programmes de niveau supérieur comprenant des cours préparatoires à des examens d'entrée sélectifs). Ces programmes d'un an sont destinés aux élèves ayant achevé le deuxième cycle de l'enseignement secondaire et souhaitant accéder à des programmes de formation professionnelle sélective de l'enseignement supérieur de cycle court. Les programmes proposent une formation dans divers domaines professionnels. Ils préparent les élèves à accéder à des écoles de services paramédicaux et sociaux de haut niveau (écoles paramédicales et sociales), dont le niveau est équivalent au Brevet de technicien supérieur (BTS) (c'est-à-dire le niveau 5 de la CITE). (CITE-P/CITE-A : 454)

Allemagne - Berufsschulen/Duales System (Programme de second cycle au système double). C'est une forme d'apprentissage spéciale (second cycle) combinant enseignement et formation, à la fois dans une école professionnelle et une entreprise. Les élèves doivent avoir achevé le deuxième cycle de l'enseignement secondaire avec succès, soit un programme général avec certification d'entrée universitaire ou un programme professionnel (dans le système double menant au Lehrabschluss ou un programme de formation professionnelle à plein temps dans une école professionnelle spécialisée), pour accéder à ce programme de 3 ou 3,5 ans. Les diplômés sont qualifiés pour poursuivre leurs études dans les Fachschulen ou accéder directement au marché du travail. (CITE-P/CITE-A : 454)

Maroc - (Technicien spécialisé). II s'agit d'un programme post-secondaire de deux ans qui nécessite l'achèvement complet du deuxième cycle de l'enseignement secondaire. II vise à préparer les techniciens à diverses professions spécialisées. À la fin de ce programme professionnel, les participants se voient décerner le Diplôme de Technicien Spécialisé (DTS). Outre le marché du travail, ce diplôme donne également accès à l'enseignement supérieur. (CITE-P/ CITE-A : 454)

Pays-Bas - WEB-specialistenopleiding, bol en bbl (Enseignement professionnel, formation spécialisée, niveau 4). Ces programmes professionnels post-secondaires sont des programmes éducatifs à temps plein, fondés sur l'école et au système double. Généralement, les élèves débutent ces programmes d'un an à 19 ans après l'achèvement du deuxième cycle de l'enseignement secondaire (sans accès à l'enseignement supérieur). Lors de l'achèvement complet du programme, les élèves reçoivent la certification de l'enseignement professionnel secondaire, niveau spécialisé (MBO niv.4: specialist), qui donne également accès à l'enseignement supérieur. (CITE-P/CITE-A : 454)

Seychelles - Programmes de diplôme. Il s'agit de programmes professionnels de trois ans proposés après l'achèvement de la première année d'études du programme de Certificat avancé de deux ans de niveau A (CITE 344). Les diplômés du " programme de diplôme " se voient décerner un certificat diplômant donnant un accès direct à l'enseignement supérieur. (CITE-P/CITE-A : 454)

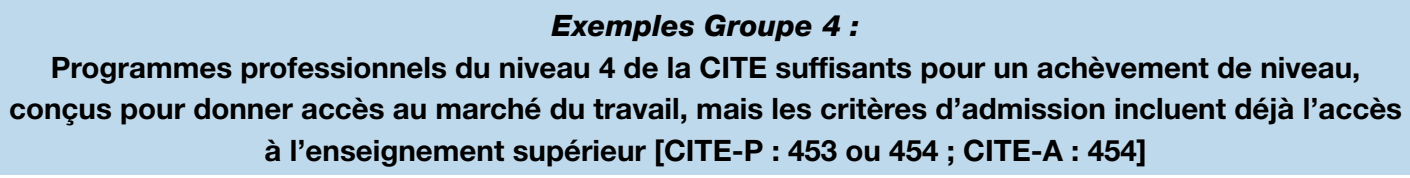

Brésil - Educação profissional de ensino médio (subsequente) (Enseignement professionnel du second cycle de l'enseignement secondaire (enseignement complémentaire)). Ces programmes sont destinés aux élèves ayant déjà achevé un programme du niveau 3 de la CITE et souhaitant obtenir un enseignement professionnel et sont conçus pour une entrée sur le marché du travail. Les programmes durent au moins 800 heures (entre 8 et 18 mois). Les élèves de ces programmes sont généralement plus âgés que ceux des programmes de la CITE 3 et recherchent un enseignement professionnel spécifique pour avoir une meilleure situation sur le marché du travail. De nombreux élèves sont déjà employés mais souhaitent se spécialiser davantage dans leur domaine. Les élèves qui accèdent à ce programme ont déjà accès à l'enseignement supérieur au travers de leur certification préalable CITE 3 (deuxième cycle de l'enseignement secondaire - ensino médio). Toutefois, ce programme professionnel est conçu spécialement pour le marché du travail et n'offre ni préparation ni accès à l'enseignement supérieur au-delà de ceux acquis dans la CITE 3. (CITE-P : 453 ; CITE-A : 454)

Grèce - Diploma epagematikis katartisis epipedou metadefterovathmias epagelmatikis katarisis (Diplôme de formation professionnelle). Ces programmes professionnels de cinq semestres (quatre semestres de cours et un semestre d'apprentissage) sont proposés par les Instituts de Formation professionnelle (Institouto Epagelmatikis Katartisis IEK) et exigent l'achèvement du deuxième cycle de l'enseignement secondaire pour y accéder. Le diplôme obtenu à l'achèvement de ces programmes ne donne pas accès à l'enseignement supérieur ou à des établissements 
universitaires. II est destiné à une entrée sur le marché du travail en tant que technicien qualifié de niveau supérieur. Parmi les critères d'admission, on trouve l'achèvement complet du deuxième cycle du secondaire (lyceia), qu'il soit général ou professionnel, qui donne accès au niveau 6 de la CITE (avec des examens d'entrée généraux à l'université). (CITE-P : 453 ; CITE-A : 454)

Portugal - Ensino pós-secundário não superior, Curso de Especialização Tecnológica (Cours de spécialisation technologique post-secondaire non-supérieur). Ces programmes à orientation professionnelle ont une durée de 1 à 1,5 ans et mènent au diplôme Especialização Tecnológica. Ces cours de spécialisation technologique sont des cours de formation post-secondaire non-supérieure qui préparent les jeunes et les adultes à exercer des emplois qualifiés, destinés à faciliter l'entrée sur le marché du travail. Les cours ont des composantes de formation à l'école et sur le lieu de travail. Le critère d'admission minimal à ces programmes est l'achèvement complet du deuxième cycle de l'enseignement secondaire. L'achèvement complet de ces programmes donne accès aux programmes universitaires de premier diplôme aux niveaux 6 et 7 de la CITE. (CITE-P/CITE-A : 454)

\section{Exemples Groupe 5 :}

Programmes professionnels du niveau 4 de la CITE suffisants pour un achèvement de niveau, avec une expérience professionnelle pertinente considérée parmi les critère d'admission [CITE-P : 453 ou 454 ; CITE-A : 453 ou 454]

Finlande - Erikoisammattitutkinto (Programmes professionnels préparant à des certifications professionnelles spécialisées). II s'agit de programmes qui préparent à une certification fondée sur les compétences ou à un examen des aptitudes que l'on passe généralement après quelques années d'expérience professionnelle (par exemple sur les aptitudes artisanales ou techniques). Si les élèves participent habituellement à ces programmes afin de se préparer à la certification, la certification peut aussi être obtenue en se fondant sur des aptitudes professionnelles plutôt que sur l'achèvement d'études préalables. Les participants à ces programmes ont généralement achevé le deuxième cycle de l'enseignement secondaire ou ont des aptitudes équivalentes. Après l'achèvement complet de ces programmes, les élèves ont également accès à l'enseignement supérieur. (CITE-P/CITE-A : 454)

Norvège - Halårig til ettårig fagskoleutdanning (programmes professionnels de 0,5 à 1 an). Ces programmes sont conçus pour une entrée directe sur le marché du travail et répondent à des besoins professionnels spécialisés. Souvent, ils constituent une spécialisation plus approfondie par rapport à des certificats d'artisan ou d'ouvrier qualifié. Parfois, ils abordent des domaines que le niveau 3 de la CITE ne prévoit pas. Le critère d'admission est l'achèvement complet du deuxième cycle de l'enseignement secondaire ou d'une formation de ce niveau (niveau 3 de la CITE). (CITE-P : 453 ; CITE-A : 453)

\section{Exemples Groupe 6 :}

Programmes professionnels du niveau 4 de la CITE durant plus de deux ans mais avec un contenu inférieur à celui de l'enseignement supérieur [CITE-P : 453 ou 454 ; CITE-A : 453 ou 454]

Guinée-Bissau - Treinamento (formação) de professores de ensino secundário geral (Formation d'enseignant pour le secondaire général). Ce programme a une durée de trois ans après l'achèvement du deuxième cycle de l'enseignement secondaire. Son objectif est de préparer des enseignants qualifiés pour le deuxième cycle du secondaire. Au terme du programme, les participants reçoivent un diplôme d'enseignement dans le secondaire général (Diploma de ensino secundário geral) qui donne accès au marché du travail et à l'enseignement supérieur. Le programme est classé dans l'enseignement post-secondaire non-supérieur (CITE 4) car au niveau national, il est considéré comme inférieur à l'enseignement supérieur. (CITE-P/CITE-A : 454)

Pologne - Policealne studium wokalno-aktorskie (École post-secondaire de la voix et des arts du spectacle). Ce programme professionnel du niveau 4 de la CITE prépare les élèves à des professions artistiques spécifiques et mène à l'octroi d'un certificat de fin d'études post-secondaires. Selon le Règlement du Ministère de la Culture et de l'héritage national, depuis l'année scolaire 2014/2015, l'enseignement proposé dans la policealne stadium wokalno-aktorskie s'organise en un cycle de trois ans. (CITE-P/CITE-A : 453) 
Programmes du niveau 4 de la CITE proposés dans des établissements atypiques pour un enseignement post-secondaire non-supérieur [Tous les codes CITE-P ou CITE-A au niveau 4 de la CITE]

République tchèque - Dalšl vzdělávání na vysoké škole: pro absolventy SŠ (Universités : la seconde certification pour les diplômés du deuxième cycle de l'enseignement secondaire). Ces cours généraux post-secondaires s'adressent à des élèves ayant achevé le deuxième cycle de l'enseignement secondaire (avec accès à l'enseignement supérieur) et sont proposés dans des universités. Les élèves débutent les cours à 19 ans ou plus et la durée est un an. Après l'achèvement, les élèves reçoivent un certificat (osvědsčeni). Le programme donne également accès à l'enseignement supérieur. (CITE-P/CITE-A : 444)

République tchèque - Pomaturitní studium (Cours post-secondaires, de type professionnel). Ces cours professionnel post-secondaires s'adressent à des élèves ayant achevé le deuxième cycle de l'enseignement secondaire (avec accès à l'enseignement supérieur). Le programme prend place dans des écoles secondaires. L'âge d'entrée typique est 19 ans et la durée est un an. Après l'achèvement, les élèves reçoivent un certificat (osve`dsčeni). Le programme donne également accès à l'enseignement supérieur. (CITE-P/CITE-A : 454)

Espagne - Títulos propios de Universidad de menos de 2 años (Diplômes spécifiques d'universités, moins de deux ans). Ces programmes d'enseignement post-secondaire diversifiés de courte durée sont proposés dans des universités et ne mènent pas à des diplômes universitaires nationaux. La durée varie de 0,5 à 1 an. Les élèves doivent avoir achevé le deuxième cycle de l'enseignement secondaire pour accéder à ces programmes professionnels. (CITE-P/CITE-A : 453)

\section{Note}

1. Les numéros de paragraphe constituent des références à la classification CITE 2011. Pour plus de détails, veuillez consulter le Guide du lecteur. 


\section{PARTIE III \\ NIVEAUX 5 À 8 DE LA CITE : ENSEIGNEMENT SUPÉRIEUR}

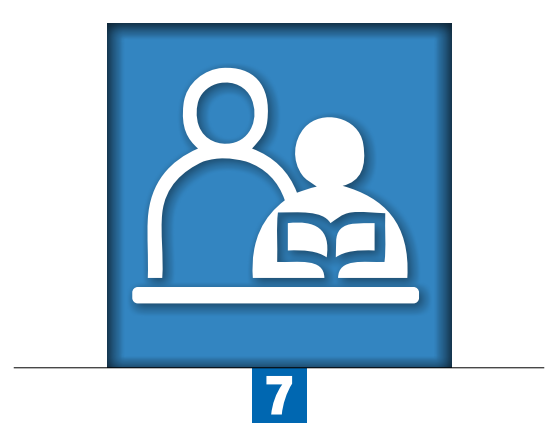

Chapitre 7

\section{Aperçu de la CITE 2011 niveaux de l'enseignement supérieur}

L'enseignement supérieur complète l'enseignement secondaire et offre des activités d'apprentissage dans des domaines d'études spécialisées. II vise un apprentissage à un niveau élevé de complexité et de spécialisation. L'enseignement supérieur comprend ce que l'on qualifie habituellement d'enseignement académique mais il comprend également l'enseignement professionnel avancé.

On observe en général une hiérarchie évidente entre les certifications décernées par les programmes de l'enseignement supérieur. II comprend les niveaux 5 (enseignement supérieur de cycle court), 6 (niveau licence ou équivalent), 7 (niveau master ou équivalent) et 8 (niveau doctorat ou équivalent) de la CITE. Le contenu des programmes de l'enseignement supérieur est plus complexe et plus avancé que celui des niveaux inférieurs de la CITE. 


\section{DESCRIPTION}

\section{Définition}

$(\S 200)^{1}$ L'enseignement supérieur complète l'enseignement secondaire et offre des activités d'apprentissage dans des domaines d'études spécialisées. II vise un apprentissage à un niveau élevé de complexité et de spécialisation. L'enseignement supérieur comprend ce que l'on qualifie habituellement d'enseignement académique mais il comprend également l'enseignement professionnel avancé. II comprend les niveaux 5, 6, 7 et 8 de la CITE, nommés respectivement enseignement supérieur de cycle court, niveau licence ou équivalent, niveau master ou équivalent et niveau doctorat ou équivalent. Le contenu des programmes de l'enseignement supérieur est plus complexe et plus avancé que celui des niveaux inférieurs de la CITE.

(§201) Les premiers programmes des niveaux 5, 6 et 7 de la CITE exigent l'achèvement complet de programmes de niveau 3 de la CITE donnant un accès direct aux premiers programmes de l'enseignement supérieur. II est également possible d'y accéder à partir du niveau 4 de la CITE. Outre les exigences liées aux certifications, l'admission dans les programmes éducatifs de ces niveaux d'enseignement peut dépendre du choix du sujet et/ ou des notes obtenues au niveau 3 ou 4 de la CITE. II peut aussi s'avérer nécessaire de passer et de réussir des examens d'entrée.

(§205) L'achèvement complet du niveau 7 de la CITE est généralement exigé pour accéder au niveau 8 de la CITE (voir figure 1).

(§203) On observe en général une hiérarchie évidente entre les certifications décernées par les programmes de l'enseignement supérieur. Toutefois, contrairement aux programmes des niveaux 1, 2, 3 et 4 de la CITE, les programmes nationaux des niveaux 5, 6 et 7 de la CITE peuvent coexister plutôt que de se présenter l'un à la suite de l'autre sous forme séquentielle.

La figure 7.1 illustre les groupes de programmes de l'enseignement supérieur (qui seront détaillés dans les chapitres suivants) et les parcours entre eux. II s'agit de :

- Programmes de l'enseignement supérieur de cycle court du niveau 5 de la CITE (voir groupe 5) ;

- Programmes de premier diplôme universitaire d'une durée cumulée théorique (au niveau de l'enseignement supérieur) de trois à quatre ans au niveau 6 de la CITE (niveau licence ou équivalent) (voir groupe 665) ;

- Programmes longs de premier diplôme universitaire d'une durée cumulée théorique (au niveau de l'enseignement supérieur) de plus de quatre ans au niveau 6 de la CITE (niveau licence ou équivalent) (voir groupe 666) ;

- Programmes de deuxième diplôme ou diplôme supplémentaire, après achèvement complet d'un programme de niveau licence ou équivalent, au niveau 6 de la CITE (niveau licence ou équivalent) (voir groupe 667) ;

- Programmes longs de premier diplôme d'une durée cumulée théorique (au niveau de l'enseignement supérieur) d'au moins cinq ans (ne nécessitant pas un enseignement supérieur préalable) au niveau 7 de la CITE (niveau master ou équivalent) (voir groupe 766) ;

- Programmes de deuxième diplôme ou diplôme supplémentaire, après achèvement complet d'un programme de niveau licence ou équivalent, au niveau 7 de la CITE (niveau master ou équivalent) (voir groupe 767) ;

- Programmes de deuxième diplôme ou diplôme supplémentaire, après achèvement complet d'un autre programme de niveau master ou équivalent, au niveau 7 de la CITE (niveau master ou équivalent) (voir groupe 768) ; et

- Programmes de niveau doctorat ou équivalent du niveau 8 de la CITE (voir groupe 8). 
- Figure 7.1 -

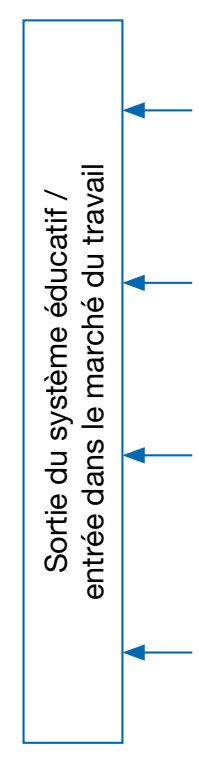

Parcours dans l'enseignement supérieur dans la CITE 2011

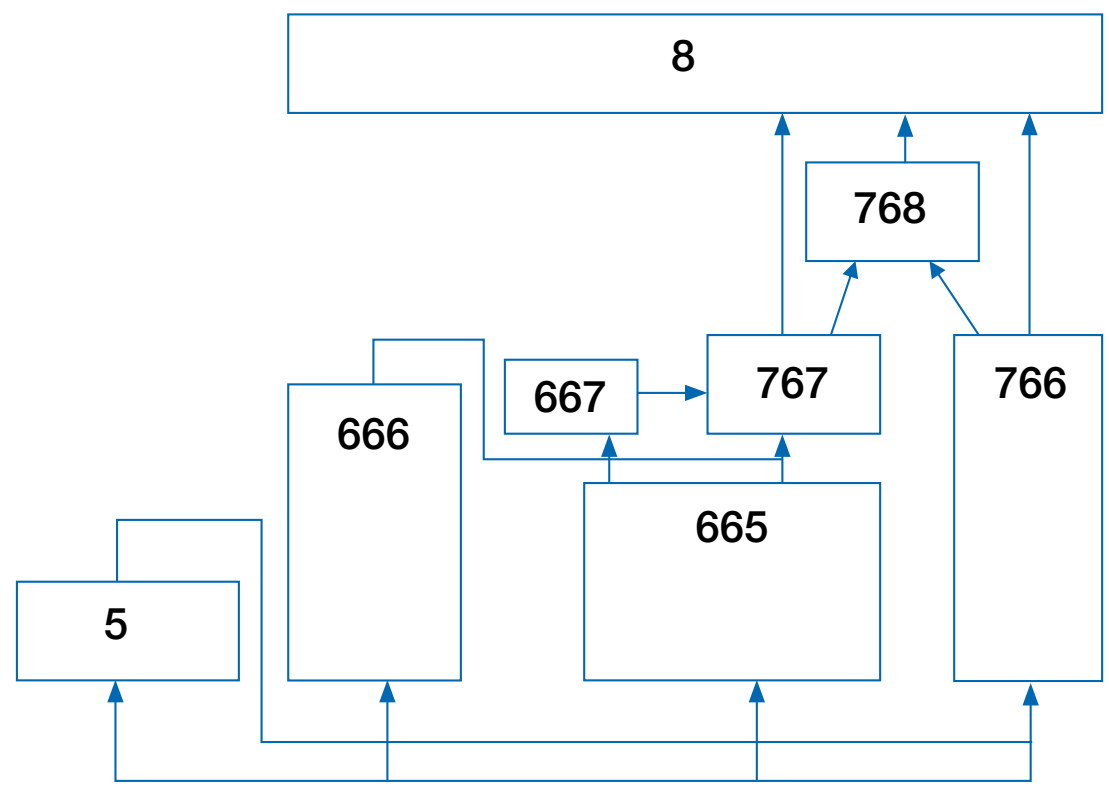

Achèvement des niveaux 3 ou 4 de la CITE avec accès direct aux premiers programmes de l'enseignement supérieur des niveaux 5,6 ou 7 de la CITE

\section{CORRESPONDANCES ENTRE LA CITE 2011 ET LA CITE 1997}

(\$281) La CITE 2011 se caractérise par quatre niveaux d'enseignement supérieur, comparés à deux niveaux dans la CITE 1997. Les niveaux 5, 6 et 7 de la CITE 2011 cumulés correspondent au niveau 5 de la CITE 1997. Le niveau 8 de la CITE 2011 correspond au niveau 6 de la CITE 1997.

Les nouveaux niveaux identifient mieux la structure actuelle des programmes de l'enseignement supérieur, notamment en opérant une distinction entre les programmes de licence et les programmes de master.

(\$282) La CITE 2011 simplifie les dimensions complémentaires des niveaux de l'enseignement supérieur comparé à la CITE 1997.

- Au niveau 5 de la CITE 2011, les programmes d'enseignement professionnel se distinguent des programmes d'enseignement général par le deuxième chiffre du code. Dans la CITE 1997, cette distinction n'existait pas. ॥ sera possible d'opérer une distinction entre les orientations académique et professionnelle aux niveaux 6 à 8 de la CITE 2011 une fois que des définitions internationalement convenues auront été élaborées.

- Aux niveaux 6 et 7 de la CITE 2011, le troisième chiffre de la classification permet de distinguer les programmes en fonction de leur durée et de leur position dans la structure nationale des diplômes et certifications. Les données rapportées par ces sous-catégories peuvent être utilisées pour le calcul de statistiques et d'indicateurs tels que le taux d'admission et d'obtention de diplômes dans l'enseignement supérieur. Dans la CITE 1997, le « type de programme » était utilisé pour subdiviser la CITE 5A entre programmes de premier diplôme et programmes de deuxième diplôme et de diplôme supplémentaire (ce qui correspond actuellement aux niveaux 6 et 7 combinés de la CITE 2011).

\section{Note}

1. Les numéros de paragraphe constituent des références à la classification CITE 2011. Pour plus de détails, veuillez consulter le Guide du lecteur. 



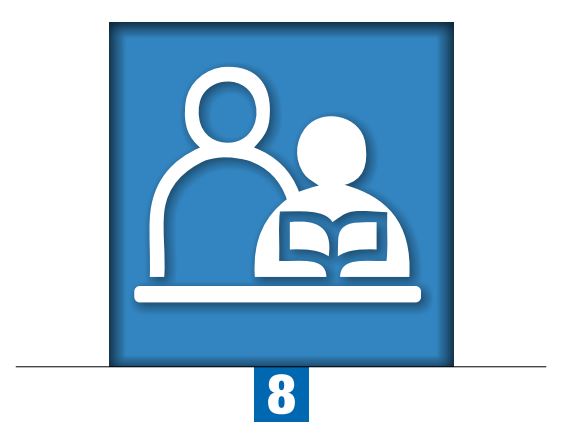

Chapitre 8

\section{Niveau 5 de la CITE 2011:}

\section{Enseignement supérieur de cycle court}

Le contenu des programmes du niveau 5 de la CITE est clairement plus complexe que celui des programmes du deuxième cycle du secondaire donnant accès à ce niveau. Les programmes du niveau 5 de la CITE sont conçus pour approfondir les connaissances en enseignant de nouveaux concepts, techniques et idées que le deuxième cycle de l'enseignement secondaire ne couvre généralement pas (alors que les programmes du niveau 4 de la CITE servent à élargir les connaissances et ne sont généralement pas beaucoup plus avancés que les programmes du niveau 3 de la CITE).

Les programmes classés au niveau 5 de la CITE peuvent être désignés de plusieurs manières : enseignement technique supérieur, community college education, formation professionnelle technique ou avancée/supérieure, associate degree ou bac+2. En vue d'une comparabilité internationale, l'expression " enseignement supérieur de cycle court » est utilisée pour qualifier le niveau 5 de la CITE. 


\section{DESCRIPTION}

\section{Définition}

$(\$ 207)^{1}$ Les programmes du niveau 5 de la CITE, ou « enseignement supérieur de cycle court », sont habituellement fondés sur la pratique, ils sont professionnellement spécifiques et préparent les étudiants à entrer sur le marché du travail. Toutefois, ils peuvent aussi représenter une passerelle vers d'autres programmes de l'enseignement supérieur (niveaux 6 ou 7 de la CITE). Peuvent également être classés au niveau 5 de la CITE certains programmes académiques de l'enseignement supérieur dont le niveau se situe en dessous des programmes de niveau licence ou équivalent.

(\$208) Les programmes du niveau 5 de la CITE ont un contenu plus complexe que les programmes des niveaux 3 et 4 de la CITE, mais ils sont plus courts et généralement moins orientés vers la théorie que les programmes de niveau 6 de la CITE.

\section{Critères de classification (\$211)}

\section{Critères principaux}

a) Contenu

(§212) Le niveau 5 de la CITE rend compte du plus bas niveau de l'enseignement supérieur. Le contenu des programmes à ce niveau est plus complexe que dans l'enseignement secondaire (niveau 3 de la CITE) ou post-secondaire non-supérieur (niveau 4 de la CITE), mais il est moins complexe que celui des programmes du niveau 6 de la CITE (niveau licence ou équivalent).

b) Critères d'admission

(§208) L'admission aux programmes de niveau 5 de la CITE exige l'achèvement complet d'un programme du niveau 3 ou 4 de la CITE donnant accès à l'enseignement supérieur.

c) Durée minimale du niveau

(§213) Les programmes du niveau 5 de la CITE durent au minimum deux ans.

\section{Critères subsidiaires}

a) Point de transition institutionnelle

(§214) Le point de transition entre les établissements d'enseignement non supérieur et ceux de l'enseignement supérieur peut aider à identifier la limite entre le niveau 3 de la CITE, le niveau 4 de la CITE, et l'enseignement supérieur. Les programmes de niveau 5 de la CITE sont souvent dispensés dans des établissements d'enseignement différents de ceux qui offrent des programmes de niveaux 6, 7 et 8 .

b) Durée habituelle du niveau

(§213) Les programmes du niveau 5 de la CITE durent au minimum deux ans et au maximum trois ans, mais il y a des exceptions. 


\section{Dimensions complémentaires}

(§216) Deux dimensions permettent de distinguer les programmes éducatifs du niveau 5 de la CITE.

(§217) Orientation du programme :

- Général ; et

- Professionnel2.

(§218) Achèvement de niveau :

- Non achèvement du niveau 5 de la CITE : programme d'une durée inférieure à deux ans (insuffisante pour un achèvement du niveau) : et

- Achèvement du niveau 5 de la CITE : programme d'une durée de deux ans ou plus (suffisante pour un achèvement du niveau).

\section{CORRESPONDANCES ENTRE LA CITE 2011 ET LA CITE 1997}

Voir la partie correspondance dans le chapitre sur l'enseignement supérieur.

\section{DIRECTIVES POUR LA CLASSIFICATION DES PROGRAMMES ET POUR LES CERTIFICATIONS RECONNUES CORRESPONDANTES AU NIVEAU 5 DE LA CITE}

Le contenu des programmes du niveau 5 de la CITE est clairement plus complexe que celui des programmes du deuxième cycle du secondaire donnant accès à ce niveau. Les programmes du niveau 5 de la CITE sont conçus pour approfondir les connaissances en enseignant de nouveaux concepts, techniques et idées que le deuxième cycle de l'enseignement secondaire ne couvre généralement pas (alors que les programmes du niveau 4 de la CITE servent à élargir les connaissances et ne sont généralement pas beaucoup plus avancés que les programmes du niveau 3 de la CITE (§191)). (Exemples Groupe 1)

Les programmes exigeant un achèvement complet d'un programme de niveau 6 ou 7 de la CITE ne doivent pas être classés dans le niveau 5 , quelle que soit leur durée.

Les programmes d'une durée inférieure à deux ans succédant immédiatement à un deuxième cycle de l'enseignement secondaire doivent normalement être classés dans le niveau 4 de la CITE sauf s'ils sont clairement directement équivalents aux premiers cycles d'autres programmes classés dans le niveau 5 de la CITE (satisfaisant les critères de classification dans le niveau 5 de la CITE, y compris le critère de durée cumulée minimale de deux ans). (Exemples Groupe 2)

Dans certains cas, une succession de programmes de courte durée peut atteindre l'équivalent de deux à trois années d'études, ce qui respecte le critère de classification dans le niveau 5 de la CITE. L'achèvement de chaque programme dans la succession peut donner lieu à une certification. Les premiers programmes de la succession seraient donc classés au niveau 5 de la CITE dans la CITE-P (« insuffisant pour un achèvement du niveau », pour autant que l'ensemble de la succession soit éligible pour une classification dans le niveau 5) et dans le niveau 4 de la CITE pour le niveau d'éducation atteint dans la CITE-A (même s'il n'existe pas de programme du niveau 4 de la CITE correspondant au niveau national).

Les programmes du niveau 5 de la CITE peuvent permettre d'obtenir des crédits transférables dans les programmes de niveau 6 ou 7 de la CITE (\$209), ce qui signifie que les participants sont exemptés de certains cours ou modules du programme de niveau 6 ou 7 de la CITE dans lequel ils passent grâce à leur achèvement complet du programme du niveau 5 de la CITE. (Exemples Groupe 3)

II faut être particulièrement attentif à la classification des programmes très longs ou des seconds programmes de l'enseignement supérieur même s'ils étaient classés dans le niveau 5B de la CITE 1997. Les seconds programmes représentant au moins cinq ans de durée cumulée au niveau 5 de la CITE doivent normalement être classés dans le niveau 6 de la CITE (sauf si la certification reconnue qui en résulte est clairement inférieure au diplôme de licence). Le niveau d'éducation atteint doit normalement correspondre au niveau 6 de la CITE même si la durée d'études dans le niveau 6 de la CITE représente moins de trois ans. Cela s'explique par le fait que la totalité des études de l'enseignement supérieur dure au moins cinq ans et que donc la complexité du contenu maîtrisé à la fin du programme doit normalement être équivalente à celle couverte par des programmes de premier diplôme de courte durée dans le niveau 6 de la CITE. 
De même, il faut être attentif dans le traitement des premiers programmes très longs (représentant au moins cinq ans d'études) qui respectent les critères de classification dans le niveau 5 de la CITE et vérifier si ce ne sont pas des programmes couvrant les niveaux 5 et 6 de la CITE. Les premiers cycles de ces programmes doivent être classés dans le niveau 5 de la CITE et les suivants dans le niveau 6 de la CITE. La détermination de la limite entre les deux niveaux devrait se fonder autant que possible sur une évaluation de la complexité du contenu des différents cycles du programme. Comme pour les seconds programmes, si la certification qui résulte du très long programme est clairement inférieure au diplôme de licence, l'ensemble du programme doit être classé au niveau 5 de la CITE malgré sa longue durée.

\section{NIVEAU D'ÉDUCATION ATTEINT AU NIVEAU 5 DE LA CITE}

Le niveau d'éducation atteint au niveau 5 de la CITE exige l'achèvement complet - menant à une certification reconnue - d'un programme qui représente une durée cumulée minimale d'au moins deux ans au niveau 5 de la CITE. Dans les pays de l'Espace européen de l'enseignement supérieur, cela représente l'équivalent d'au moins 120 crédits du Système européen de transfert de crédits.

L'achèvement complet de programmes représentant moins de deux ans d'études dans le niveau 5 de la CITE mène normalement à un niveau d'éducation atteint correspondant au niveau 4 de la CITE (même dans les pays ne disposant pas de programmes de niveau 4 de la CITE). (Exemples Groupe 4)

Lorsque des programmes de différentes durées mènent à des certifications reconnues comme équivalentes, ils peuvent normalement être classés dans le même niveau de la CITE. ${ }^{3}$ De même, deux ou plusieurs certifications différentes mais équivalentes, doivent normalement donner un niveau d'éducation atteint dans le même niveau de la CITE mais pas nécessairement avec le même code (par exemple si l'une est générale et l'autre professionnelle, les codes CITE-A pourraient être 540 et 550 respectivement). De plus, quand un programme éducatif dure moins de deux ans (par exemple quand il appartient à une succession de programmes) mais que la certification obtenue est équivalente à d'autres certifications classées au niveau 5 de la CITE, le programme et la certification doivent être classés dans le niveau 5 de la CITE. (Exemples Groupe 5)

(§221) En ce qui concerne le niveau d'éducation atteint, les certifications intermédiaires reconnues obtenues lors de l'achèvement complet de programmes considérés comme insuffisants pour être pris en compte comme achèvement du niveau 5 de la CITE sont classées dans le niveau 4 de la CITE.

(§222) Les certifications intermédiaires reconnues obtenues lors de l'achèvement complet de programmes (avant le premier diplôme) considérés comme insuffisants pour être pris en compte comme achèvement du niveau 6 de la CITE, sont classées dans le niveau 5 de la CITE pour ce qui est du niveau d'éducation atteint.

L'achèvement complet de premiers programmes de l'enseignement supérieur représentant moins de trois ans d'études dans le niveau 6 de la CITE mène à un niveau d'éducation atteint correspondant au niveau 5 de la CITE (même dans les pays ne disposant pas de programmes de niveau 5 de la CITE). Voir Exemples Groupe 4 dans le chapitre sur le niveau 6 de la CITE.

\section{EXEMPLES DE PROGRAMMES ET DE CERTIFICATIONS CLASSÉS AU NIVEAU 5 DE LA CITE}

(\$210) Les programmes classés au niveau 5 de la CITE peuvent être désignés de plusieurs manières : enseignement technique supérieur, community college education, formation professionnelle technique ou avancée/supérieure, associate degree ou bac+2. En vue d'une comparabilité internationale, l'expression « enseignement supérieur de cycle court » est utilisée pour qualifier le niveau 5 de la CITE.

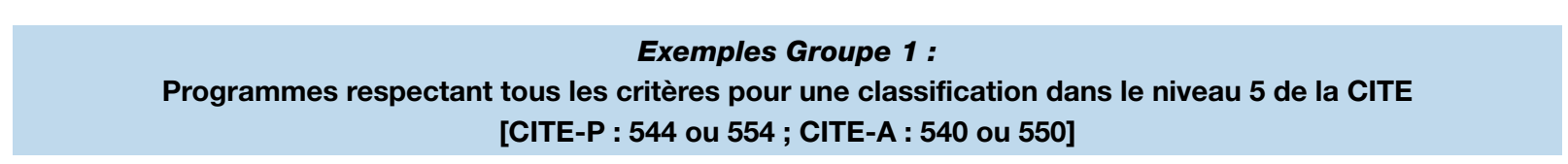

Australie - Cours para-professionnels/techniques. Ces cours sont conçus pour fournir un enseignement initial ou une formation initiale en vue de développer l'étendue des aptitudes spécialisées requises pour l'emploi dans les professions 
para-professionnelles. Les diplômes fréquents sont le Diplôme associé ou le Certificat avancé et les critères d'admission spécifient habituellement que les personnes qui entrent dans le programme détiennent un Certificat (CITE 3) dans le domaine pertinent. Les cours ont généralement une durée de l'ordre de deux années en équivalent temps plein. (CITE-P : 554 ; CITE-A : 550).

Brunéi Darussalam - Diplôme national supérieur (DNS). II s'agit d'un programme professionnel de 2,5 ans nécessitant l'achèvement d'un programme du deuxième cycle de l'enseignement secondaire avec accès à l'enseignement supérieur, comme le niveau A, le Diplôme national (DN) ou le Sijil Tinggi Pelajaran Ugama (STPU) des écoles religieuses. (CITE-P : 554 ; CITE-A : 550).

Cambodge - Diplôme associé. II s'agit d'un programme de deux ans nécessitant l'achèvement de l'Année d'études 12 ou d'un certificat technique/professionnel 3 (tous deux du niveau 3 de la CITE). Le programme mène à des diplômes associés dans le tourisme et l'hôtellerie, l'infirmerie et la technologie informatique. (CITE-P : 554 ; CITE-A : 550).

France - Enseignement conduisant aux Brevets de techniciens supérieurs et équivalent. II s'agit d'un programme de deux ans menant au Brevet de technicien supérieur (BTS). Le critère d'admission est le baccalauréat ou le brevet de technicien complété par des notes suffisantes et des recommandations des enseignants. Les détenteurs de BTS peuvent, dans certaines conditions, poursuivre leurs études à l'université ou dans des établissements d'enseignement supérieur. Cette certification est au même niveau que le diplôme universitaire de technologie (DUT), bien qu'elle soit plus spécialisée et offre moins d'opportunités pour la poursuite des études. (CITE-P : 554 ; CITE-A : 550).

Japon - Koutou senmon gakko (Collèges de Technologie, années 4-5). II s'agit des deux dernières années du programme d'enseignement professionnel de cinq ans, le Koutou senmon gakko. Les étudiants suivent principalement un enseignement d'ingénierie spécialisée. Après avoir achevé le programme avec succès et avoir réussi un examen d'entrée, les étudiants peuvent accéder à la troisième année de programmes universitaires ou progresser vers le Koutou senmon gakko Senkoka (Cours d'ingénierie avancé). (CITE-P : 554 ; CITE-A : 550).

Mauritanie - Brevet de technicien supérieur. Ce programme de trois ans nécessite l'achèvement du Baccalauréat national (deuxième cycle de l'enseignement secondaire). À la fin du programme, les diplômés reçoivent le certificat de technicien supérieur. (CITE-P : 554 ; CITE-A : 550).

Mexique - Technicien supérieur (Enseignement technique professionnel). Ces programmes sont offerts dans des Instituts technologiques et mènent au Certificat professionnel associé. Les diplômés de ces programmes de deux ans sont considérés comme des techniciens qualifiés. (CITE-P : 554 ; CITE-A : 550).

Norvège - Toårige fagskoleutdanning (Enseignement professionnel de niveau supérieur). Ces programmes professionnels de deux ans sont conçus pour répondre aux exigences de maître artisan, de techniciens qualifiés ou de professions para-professionnelles. Ils donnent une entrée directe sur le marché du travail. Les diplômés d'études techniques, qui constituent la majorité des diplômés à ce niveau, peuvent bénéficier d'une exemption allant jusqu'à un an du programme de licence en ingénierie. Le critère d'admission est l'achèvement complet du deuxième cycle de l'enseignement secondaire ou d'une formation de ce niveau (niveau 3 de la CITE). (CITE-P : 554 ; CITE-A : 550).

Arabie saoudite - (Programmes de diplôme général intermédiaire). Ces programmes sont proposés dans des collèges communautaires à des diplômés du deuxième cycle du secondaire dans de nombreuses matières. À la fin de ces programmes de deux ans, les diplômés se voient décerner le diplôme général intermédiaire dans le domaine d'études respectif. (CITE-P : 544 ; CITE-A : 540).

Espagne - Ciclos Formativos de Formación Profesional de Grado Superior (Formation professionnelle - niveau avancé). Ces programmes d'une durée de deux ans sont compris dans le Cadre de l'enseignement supérieur espagnol. La certification obtenue à l'achèvement de ce programme, le Técnico Superior (Formation professionnelle - niveau avancé Certificat et Titre), est équivalente à celle de technicien qualifié dans une profession donnée. L'admission se fonde sur l'achèvement complet du bachiller (niveau 3 de la CITE). (CITE-P : 554 ; CITE-A : 550).

Ukraine - Неповна вища освіта (Achèvement partiel de l'enseignement supérieur). Ce sont des programmes de deux à trois ans dispensés dans des établissements d'enseignement supérieur. Le critère d'admission minimal est le certificat d'achèvement du deuxième cycle de l'enseignement secondaire. À l'achèvement du programme, les diplômés reçoivent le диплом молодшого спеиіiаліста (diplôme de spécialiste junior). (CITE-P : 554 ; CITE-A : 550). 


\section{Exemples Groupe 2 : \\ Programmes de courte durée mais équivalents aux premiers cycles d'un autre programme du niveau 5 de la CITE [CITE-P : 541 ou 551 ; CITE-A : 444 ou 454]}

Royaume-Uni - Certificat national supérieur, Higher National Certificate (HNC). Pour être admis dans ce programme, les participants doivent avoir au moins 18 ans et posséder une certification nationale appropriée décernée par le Business and Technical Education Council (BTEC) ou équivalent ou un GCE A-level (certifications CITE 3). L'objectif du programme est de développer des aptitudes et de donner une formation qui mènera à de nombreuses activités professionnelles. ॥ est proposé dans des colleges, certaines universités et quelques centres de formation. Les HNC sont plus courts que les Higher National Diplomas (HND) et durent environ un an à temps plein. Les détenteurs de HNC peuvent accéder à la seconde année des programmes HND et les détenteurs de certains HNC peuvent accéder à la seconde année d'un programme de licence. Ce programme est classé au niveau 5 de la CITE. Le niveau d'éducation atteint avec la certification qui en résulte est l'achèvement du niveau 4 de la CITE (la durée du programme est en effet trop courte - un an - pour l'achèvement du niveau 5 de la CITE [CITE-P : 551 ; CITE-A : 454).

\section{Exemples Groupe 3 : \\ Programmes classés au niveau 5 de la CITE permettant d'obtenir des crédits transférables dans la poursuite d'études aux niveaux 6 ou 7 de la CITE [CITE-P : 544 ou 554 ; CITE-A : 540 ou 550]}

France - Enseignement en institut universitaire de technologie (IUT). II s'agit d'un programme de deux ans axé sur la technologie menant au Diplôme universitaire de technologie (DUT). Les détenteurs d'un DUT peuvent poursuivre leurs études au niveau universitaire en vue d'obtenir la licence (une certification de niveau 6 de la CITE), bien que le programme soit principalement destiné à préparer les étudiants à une entrée directe sur le marché du travail. Le critère d'admission est le baccalauréat complété par des notes suffisantes et des recommandations des enseignants. (CITE-P : 554 ; CITE-A : 550).

Japon - Senmon gakko (College de formation professionnelle). Ce programme offre principalement une formation professionnelle. Le critère d'admission minimal est l'achèvement complet du deuxième cycle de l'enseignement secondaire (niveau 3 de la CITE). Après avoir achevé le programme et satisfait d'autres exigences spécifiques, un étudiant peut obtenir un Diplôme (durée d'au moins deux ans) ou un Diplôme avancé (durée d'au moins quatre ans). En général, l'étudiant reçoit simultanément les certifications permettant d'entrer à l'université (niveau 6 de la CITE ou 766) ou à l'école supérieure (niveau 7 de la CITE). (CITE-P : 554 ; CITE-A : 550).

Liban - (Programmes d'Excellence technique). II s'agit de programmes de deux ans proposés dans des instituts techniques et professionnels aux détenteurs de baccalauréats professionnels ou généraux (deuxième cycle de l'enseignement secondaire). À la fin de ces programmes, les participants reçoivent le certificat d'excellence technique, Technique Supérieur (T.S.), destiné à préparer les techniciens qualifiés au marché du travail. Cette certification est également considérée comme le critère d'admission au programme de Licence technique, qui est classé au niveau licence (niveau 6 de la CITE). (CITE-P : 554 ; CITE-A : 550).

Soudan - (Programmes de diplôme technique). Il s'agit de programmes de trois ans proposés dans différentes universités nationales dans plusieurs domaines d'études. Après leur achèvement, les participants se voient décerner le certificat de diplôme technique. Bien que ce certificat soit principalement destiné au marché du travail il peut, parallèlement à une expérience professionnelle pertinente, permettre d'obtenir des crédits pour des études ultérieures au niveau licence (niveau 6 de la CITE). (CITE-P : 554 ; CITE-A : 550).

Timor-Leste - Politecnic Diploma 2 (Programmes professionnels, diplôme polytechnique 2). Ces programmes professionnels de deux ans exigent l'achèvement du deuxième cycle de l'enseignement secondaire (avec accès à l'enseignement supérieur). À l'achèvement de ces programmes, les diplômés reçoivent le Diploma 2, qui permet d'obtenir les crédits pour de futurs programmes éducatifs, dont certains sont de niveau licence. (CITE-P : 554 ; CITE-A : 550 ).

Royaume-Uni - Diplôme national supérieur, Higher National Diploma (HND). Pour être admis dans ce programme, les participants doivent avoir au moins 18 ans et posséder une certification nationale appropriée décernée par le Business and Technical Education Council (BTEC) ou équivalent ou un GCE A-level (certifications CITE 3). L'objectif est de développer des aptitudes et de donner une formation qui mènera à de nombreuses activités professionnelles. II est proposé dans des colleges, certaines universités et quelques centres de formation et mène généralement au niveau 
de technicien supérieur ou de junior manager. La durée est soit deux ans à plein temps ou trois ans à temps partiel. Les détenteurs de certains HND peuvent accéder à la deuxième ou à la troisième année d'un programme de licence. (CITE-P : 554 ; CITE-A : 550).

\section{Exemples Groupe 4 :}

Programmes du niveau 5 de la CITE insuffisants pour un achèvement de niveau, avec donc un niveau d'éducation atteint équivalent au niveau 4 de la CITE [CITE-P : 541 ou 551 ; CITE-A : 444 ou 454]

Danemark - Merkonom, teknonom, datanom, revision (Enseignement supérieur de cycle court, éducation ouverte des adultes). Le critère d'admission de ce programme professionnel est le deuxième cycle de l'enseignement secondaire (niveau 3 de la CITE) donnant accès à l'enseignement supérieur. Le programme dure un an et le contenu est similaire à la première année de programmes d'enseignement supérieur de cycle court de deux ans classés CITE 5 dans le pays. Comme il est plus court que ces programmes, il donne un niveau d'éducation atteint au niveau 4 de la CITE. (CITE-P : 551 ; CITE-A : 454).

Royaume-Uni - Certificat national supérieur, Higher National Certificate (HNC). Ce programme est classé au niveau 5 de la CITE (insuffisant pour un achèvement du niveau) pour les raisons décrites dans les Exemples Groupe 2 ci-dessus. Le niveau d'éducation atteint avec la certification qui en résulte est l'achèvement du niveau 4 de la CITE. (CITE-P : 551 ; CITE-A : 454).

\section{Exemples Groupe 5 : \\ Programmes de moins de deux anx menant au niveau d'éducation atteint au niveau 5 de la CITE [CITE-P: 544 ou 554; CITE-A: 540 ou 550]}

République slovaque ${ }^{4}$ - Tančné konzervatórium - 8 ročné štúdium (Conservatoire de danse - huit ans). II s'agit d'un programme de huit ans débutant à 10 ans et visant à former des danseurs professionnels et à leur procurer le certificat de fin d'études (maturité, une certification du niveau 3 de la CITE) - vysvedčenie o maturitnej skúške - et un diplôme d'étudiant sortant - absolventský diplom - qui est une certification de niveau 5 de la CITE permettant à leurs détenteurs d'enseigner la danse dans l'école fondamentale des arts. Le programme couvre les niveaux 2, 3 et 5 de la CITE, les Années d'études 1 à 4 étant classées au niveau 2 de la CITE et les Années d'études 5 à 7 au niveau 3 de la CITE et l'Année d'études 8 au niveau 5 de la CITE. La certification absolventský diplom est également décernée à la fin de deux autres programmes respectant tous les critères du niveau 5 de la CITE : pomaturitné špecializačné štúdium (étude spécialisée post-secondaire) et vyššie odborné štúdium (études professionnelles supérieures), des programmes de deux et trois ans respectivement suivant l'achèvement complet du deuxième cycle de l'enseignement secondaire. L'achèvement complet de la $8 \mathrm{e}$ année d'études du tanečné konzervatórium mène à un niveau d'éducation atteint au niveau 5 de la CITE. Bien qu'une seule année du programme soit classée au niveau 5 de la CITE, il mène à la même certification que celle décernée à la fin d'autres programmes du niveau 5 de la CITE. (CITE-P : 554 ; CITE-A : 550).

\section{Notes}

1. Les numéros de paragraphe constituent des références à la classification CITE 2011. Pour plus de détails, veuillez consulter le Guide du lecteur.

2. Une fois que les définitions des programmes « académiques » et " professionnels " pour les niveaux d'enseignement supérieur de la CITE auront été établies au niveau international, elles seront utilisées pour les catégories d'orientation du niveau 5 de la CITE plutôt que le « général » et le « professionnel ».

3. Notez que des certifications portant la même dénomination ne sont pas nécessairement équivalentes, surtout quand elles sont décernées dans des domaines d'études très différents ou quand la dénomination de la certification est très générale (par ex. certificat, diplôme, etc.).

4. L'Institut de statistique de I'UNESCO (ISU) et l'Union européenne (UE) utilisent "Slovaquie " comme dénomination officielle pour la "République slovaque ». 



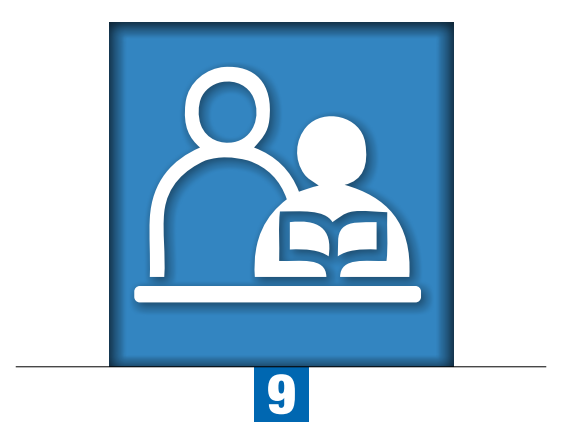

Chapitre 9

\section{Niveau 6 de la CITE 2011:}

\section{Niveau licence ou équivalent}

Les programmes du niveau 6 de la CITE, ou niveau licence ou équivalent, sont plus longs et généralement plus orientés vers la théorie que les programmes de niveau 5 de la CITE. Ils sont souvent destinés à enseigner aux participants des connaissances, aptitudes et compétences académiques et/ou professionnelles intermédiaires conduisant à un premier diplôme ou une certification équivalente.

Ils durent généralement trois à quatre ans d'études à plein temps au niveau de l'enseignement supérieur. Ils peuvent comporter un cursus pratique et/ou des périodes de stage ainsi que des cours théoriques. Ils sont traditionnellement dispensés dans des universités et des établissements d'enseignement supérieur équivalents.

Les programmes classés au niveau 6 de la CITE peuvent être désignés de plusieurs manières, comme par exemple : Bachelor's programme, licence ou premier cycle universitaire. En vue de la comparabilité internationale, l'expression " niveau licence ou équivalent » est utilisée pour qualifier le niveau 6 de la CITE. 


\section{DESCRIPTION}

\section{Définition}

$(\S 224)^{1}$ Les premiers programmes du niveau 6 de la CITE, ou niveau licence ou équivalent, sont plus longs et généralement plus orientés vers la théorie que les programmes de niveau 5 de la CITE. lls sont souvent destinés à enseigner aux participants des connaissances, aptitudes et compétences académiques et/ou professionnelles intermédiaires conduisant à un premier diplôme ou une certification équivalente. Les programmes de ce niveau se fondent généralement sur la théorie mais ils peuvent inclure un cursus pratique ; ils se fondent également sur des travaux de recherche de pointe et/ou les meilleures pratiques professionnelles. Les programmes du niveau 6 de la CITE n'imposent pas nécessairement la rédaction d'une thèse ou d'un mémoire.

(§226) Suite à l'achèvement des programmes de niveau 6 de la CITE, il est possible de poursuivre au niveau 7 de la CITE (enseignement de niveau master ou équivalent) même si tous les programmes de niveau 6 de la CITE ne donnent pas accès au niveau 7 de la CITE. Les programmes de niveau 6 de la CITE ne donnent habituellement pas un accès direct au niveau 8 de la CITE (niveau doctorat ou équivalent).

\section{Critères de classification (\$228)}

\section{Critères principaux}

a) Contenu fondé sur la théorie et/ou la pratique professionnelle

(§224) Les programmes de ce niveau se fondent généralement sur la théorie mais ils peuvent inclure un cursus pratique; ils se fondent également sur des travaux de recherche de pointe et/ou les meilleures pratiques professionnelles.

b) Critères d'admission

(§226) L'admission à ces programmes exige normalement l'achèvement complet d'un programme du niveau 3 ou 4 de la CITE donnant accès à l'enseignement supérieur.

c) Durée cumulée minimale du programme (de premier diplôme)

(§229) Les programmes de $1^{\text {er }}$ diplôme de ce niveau ont généralement une durée cumulée de trois ans d'études à plein temps dans l'enseignement supérieur. Concernant les systèmes éducatifs dans lesquels les diplômes sont accordés sur la base de cumul de crédits, une durée et une intensité comparables doivent être exigées.

d) Position dans la structure nationale des diplômes et des certifications

(§230) Les programmes de ce niveau mènent en règle générale aux premiers diplômes. Les programmes menant à un deuxième diplôme ou un diplôme supplémentaire peuvent être inclus au niveau 6 de la CITE s'ils sont équivalents en termes de complexité de contenu à des programmes déjà classés à ce niveau dans le même système éducatif et s'ils remplissent les autres critères principaux.

\section{Critères subsidiaires}

a) Qualifications du personnel

(§225) L'éducation à ce niveau prend souvent la forme de cours magistraux donnés par des enseignants qui doivent généralement avoir achevé le niveau 7 ou 8 de la CITE ou avoir accumulé assez d'expérience pour être considérés comme des experts dans leur domaine. (\$231) Le cas échéant, les certifications de niveau 8 de la CITE exigées pour certains membres du personnel enseignant peuvent représenter un bon critère subsidiaire pour les programmes éducatifs de ce niveau d'enseignement dans les systèmes éducatifs où cette exigence existe. Ceci permet de distinguer les programmes du niveau 5 de la CITE des programmes du niveau 6 de la CITE. 
b) Pas d'accès direct aux programmes de niveau 8 de la CITE

(\$225) Les programmes de ce niveau ne conduisent pas nécessairement à la réalisation d’un projet de recherche ou d'un mémoire mais si c'est le cas, ils sont moins spécialisés, moins indépendants ou bénéficient de plus de supervision que ceux du niveau 7 ou 8 de la CITE. (\$226) Les programmes de niveau 6 de la CITE ne donnent habituellement pas un accès direct au niveau 8 de la CITE (niveau doctorat ou équivalent).

\section{Dimensions complémentaires}

(§233) Deux dimensions permettent de distinguer les programmes éducatifs du niveau 6 de la CITE.

(§234) Orientation du programme :

- Académique ; et

- Professionnel².

(§235) Durée du programme et position dans la structure nationale des diplômes et certifications :

- Cycle (ou programme) de premier diplôme universitaire au niveau licence ou équivalent d'une durée cumulée théorique (au niveau de l'enseignement supérieur) de moins de trois ans et donc insuffisant pour un achèvement du niveau 6 de la CITE ;

- Programme de premier diplôme universitaire d'une durée cumulée théorique (au niveau de l'enseignement supérieur) de trois à quatre ans au niveau 6 de la CITE (niveau licence ou équivalent) ;

- Programme long de premier diplôme universitaire d'une durée cumulée théorique (au niveau de l'enseignement supérieur) de plus de quatre ans au niveau 6 de la CITE (niveau licence ou équivalent) ; et

- Programme de deuxième diplôme ou diplôme supplémentaire, après achèvement complet d'un programme de niveau licence ou équivalent, au niveau 6 de la CITE (niveau licence ou équivalent).

\section{CORRESPONDANCES ENTRE LA CITE 2011 ET LA CITE 1997}

Voir la section correspondance dans le chapitre sur l'enseignement supérieur.

\section{DIRECTIVES POUR LA CLASSIFICATION DES PROGRAMMES ET DE LEURS CERTIFICATIONS RECONNUES CORRESPONDANTES AU NIVEAU 6 DE LA CITE}

Les programmes du niveau 6 de la CITE, ou niveau licence ou équivalent, sont plus longs et généralement plus orientés vers la théorie que les programmes de niveau 5 de la CITE. Ils sont souvent destinés à enseigner aux participants des connaissances, aptitudes et compétences académiques et/ou professionnelles intermédiaires conduisant à un premier diplôme ou une certification équivalente (\$224).

Ils peuvent comporter un cursus pratique et/ou des périodes de stage ainsi que des cours théoriques (\$230).

Ils sont traditionnellement dispensés dans des universités et des établissements d'enseignement supérieur équivalents (\$224).

II est important de noter que les programmes portant un nom similaire à « licence » ne doivent être inclus dans le niveau 6 de la CITE que si la complexité de leur contenu est équivalente et s’ils répondent aux autres critères principaux (\$227).

L'admission à ces premiers programmes de niveau licence ou équivalent exige normalement l'achèvement complet d'un programme du niveau 3 ou 4 de la CITE donnant accès à l'enseignement supérieur. L'admission peut dépendre du choix du sujet et/ou des notes obtenues au niveau 3 ou 4 de la CITE. II peut aussi s'avérer nécessaire de passer et de réussir des examens d'entrée. L'admission ou le transfert au niveau 6 de la CITE est parfois possible après l'achèvement du niveau 5 de la CITE (§226) (voir Exemples Groupe 3 au niveau 5 de la CITE).

Les programmes de ce niveau mènent en règle générale aux premiers diplômes et à des certifications équivalentes dans l'enseignement supérieur (même si certains individus peuvent avoir obtenu une certification du niveau 5 de la CITE avant 
de s'engager dans un programme de niveau 6) (\$230). Ils durent généralement trois à quatre ans d'études à plein temps au niveau de l'enseignement supérieur. Concernant les systèmes éducatifs dans lesquels les diplômes sont accordés sur la base de cumul de crédits, une durée et une intensité comparables doivent être exigées (§229). (Exemples Groupe 1)

Les programmes longs d'une durée de plus de quatre ans débouchant sur un premier diplôme sont inclus dans ce niveau s'ils sont équivalents aux programmes de niveau licence en termes de complexité du contenu (\$230). Exemples

Groupe 2. Toutefois, les premiers diplômes longs dans des domaines professionnels hautement spécialisés (par exemple la médecine, la dentisterie, les études vétérinaires et, dans certains cas, le droit ou l'ingénierie) sont généralement classés au niveau 7 de la CITE (\$247). Ils sont pour la plupart nettement plus longs que d'autres premiers diplômes classés au niveau 6 de la CITE et couvrent - du point de vue de la largeur et de la profondeur - un contenu plus complexe, davantage similaire à un niveau master ou équivalent.

Les programmes nécessitant une licence pour leur admission doivent être classés comme des deuxièmes diplômes ou diplômes supplémentaires (même s'ils ne mènent pas à un diplôme). À ce niveau, de tels programmes durent généralement de un à deux ans et sont souvent orientés vers une profession, avec un degré de spécialisation plus élevé qu'un programme de premier diplôme mais n'incluent pas un contenu significativement plus complexe (§230).

Exemples Groupe 3. Les programmes qui succèdent à une licence et ont une composante importante de recherche doivent normalement être classés au niveau 7 de la CITE.

Suite à l'achèvement des programmes de niveau 6 de la CITE, il est possible de poursuivre au niveau 7 de la CITE (enseignement de niveau master ou équivalent) même si tous les programmes de niveau 6 de la CITE ne donnent pas accès au niveau 7 de la CITE (\$226). Les programmes exigeant un achèvement complet d'un programme de niveau 7 de la CITE pour leur entrée ne doivent pas être classés dans le niveau 6, quelle que soit leur durée.

\section{NIVEAU D'ÉDUCATION ATTEINT AU NIVEAU 6 DE LA CITE}

Le niveau d'éducation atteint au niveau 6 de la CITE exige l'achèvement complet - menant à une certification reconnue - d'un programme qui représente une durée minimale d'au moins trois ans au niveau 6 de la CITE. Dans les pays de l'Espace européen de l'enseignement supérieur, cela représente l'équivalent d'au moins 180 crédits du Système européen de transfert de crédits.

(\$238) En ce qui concerne le niveau d'éducation atteint, les certifications intermédiaires reconnues obtenues lors de l'achèvement complet de cycles de programmes (avant le premier diplôme) considérés comme insuffisants pour être pris en compte comme achèvement du niveau 6 de la CITE sont classées dans le niveau 5 de la CITE.

L'achèvement complet de programmes représentant moins de trois ans d'études dans le niveau 6 de la CITE mène normalement à un niveau d'éducation atteint correspondant au niveau 5 de la CITE (même dans les pays ne disposant pas de programmes de niveau 5 de la CITE). Exemples Groupe 4.

(\$239) Les certifications intermédiaires reconnues obtenues lors de l'achèvement complet de cycles de programmes (avant le premier diplôme) considérés comme insuffisants pour être pris en compte comme achèvement du niveau 7 de la CITE sont classées dans le niveau 6 de la CITE pour le niveau d'éducation atteint.

Les diplômes honoris causa décernés par les universités sur la base d'autres considérations et non de travaux de recherche ne sont pas reconnus pour la détermination du niveau d'éducation atteint.

\section{EXEMPLES DE PROGRAMMES ET DE CERTIFICATIONS CLASSÉS AU NIVEAU 6 DE LA CITE}

(\$227) Les programmes classés au niveau 6 de la CITE peuvent être désignés de plusieurs manières, comme par exemple: Bachelor's programme, licence ou premier cycle universitaire. Toutefois, il est important de noter que les programmes portant un nom similaire à « licence » ne doivent être inclus dans le niveau 6 de la CITE que s'ils répondent aux critères décrits dans le paragraphe 228 de la CITE 2011. En vue de la comparabilité internationale, l'expression « niveau licence ou équivalent » est utilisée pour qualifier le niveau 6 de la CITE. 


\section{Exemples Groupe 1 : \\ Programmes de premier diplôme de niveau licence ou équivalent (trois à quatre ans) [CITE-P : 645,655 ou 665 ; CITE-A : 640,650 ou 660 ]}

Djibouti - Enseignement supérieur de cycle long. Ce programme universitaire de quatre ans exige l'achèvement du deuxième cycle de l'enseignement secondaire avec accès à l'enseignement supérieur comme critère minimal d'admission. Après son achèvement, les étudiants sortants reçoivent un diplôme de licence ou équivalent (Licence ou Maîtrise) dans le domaine d'études pertinent. (CITE-P : 665 ; CITE-A : 660).

Finlande - Ammattikorkeakoulututkinnot (Programme de licence polytechnique). Ces programmes (d'une durée de trois à quatre ans) préparent à des professions avec des exigences d'aptitudes élevées. Ils combinent des études théoriques (études de base et professionnelles) avec un travail et une formation pratique. Les programmes impliquent l'achèvement d'un vaste projet de recherche ou d'une thèse. Les étudiants doivent avoir achevé le deuxième cycle de l'enseignement secondaire avant leur entrée. (CITE-P : 665 ; CITE-A : 660).

Allemagne - Fachhochschulen (Universités de Sciences appliquées). II s'agit d'un programme de quatre ans au niveau universitaire préparant à des professions nécessitant l'application de découvertes et de méthodes scientifiques. Les étudiants doivent avoir achevé au moins la Fachoberschule (CITE 3 ou 4) ou équivalent. II mène à un premier diplôme de l'enseignement supérieur, Diplom (FH). (CITE-P : 645 ; CITE-A : 640).

Japon - Koutou senmon gakko Senkoka, Tokurei Tekiyou Senkoka (Collège de Technologie, cours avancé validé NIAD-UE). Ce programme suit l'achèvement complet du programme de cinq ans Koutou senmon gakko (Collèges de Technologie) (trois premières années : CITE-P 354, deux dernières années : CITE-P 554). Bien que légalement ces programmes doivent avoir une durée minimale d'au moins un an, tous les programmes Koutou senmon gakko Senkoka se prolongent plus de deux ans et procurent un enseignement d'ingénierie avancé-spécialisé. Les étudiants qui achèvent le Tokurei Tekiyou Senkoka (Collège de Technologie, cours avancé validé NIAD-UE) avec succès, qui est reconnu comme équivalent à un programme de premier cycle par l'Institution nationale des diplômes académiques et évaluation universitaire (NIAD-UE), peuvent se voir décerner une licence et progresser vers une école supérieure. (CITE-P : 665 ; CITE-A : 660).

Kyrgyzstan - Высшее профессиональное образование, ведущее к поступлению в программы продвинутых научных исследований и получению диплома бакалавра (Programme éducatif de licence). II s'agit d'un programme d'enseignement supérieur de quatre ans qui nécessite le certificat du deuxième cycle de l'enseignement secondaire pour son entrée. Après l'achèvement complet, les sortants se voient décerner une licence dans le domaine d'études pertinent. (CITE-P : 665 ; CITE-A : 660).

Pays-Bas - Hoger beroepsonderwijs (HBO) (Programmes professionnels de licence). Les programmes HBO donnent une formation théorique et pratique à des professions nécessitant une certification professionnelle supérieure. Dans ces programmes d'enseignement professionnel de quatre ans, l'apprentissage passe davantage par la pratique que dans les universités. Les matières le plus souvent étudiées sont l'agriculture, la formation des enseignants, le travail social et l'enseignement communautaire, les soins de santé et les arts. (CITE-P : 655 ; CITE-A : 650).

Espagne - Diplomatura Universitaria (Enseignement universitaire - premier diplôme (premier cycle)). ॥ s'agit d'un programme de premier diplôme universitaire de trois ans conduisant au Diplomado Universitario, Arquitecto Técnico or Ingeniero Técnico dans un domaine spécifique. Les détenteurs de ces certifications peuvent accéder directement au marché du travail ou être admis dans le second cycle de l'enseignement universitaire. (CITE-P : 665 ; CITE-A : 660).

Suisse - Fachhochschule, haute école spécialisée (Programme de licence). Pour accéder à ces programmes de trois à quatre ans, il faut une «Berufsmaturität/maturité professionnelle » (enseignement professionnel CITE 3 d'une durée de trois ou quatre ans avec une composante d'enseignement général significativement élargie). Ils préparent les étudiants à des métiers hautement qualifiés dans des domaines d'études tels que l'architecture, l'ingénierie, l'administration des affaires, le design, la santé, le travail social, les arts et l'enseignement. (CITE-P : 645 ; CITE-A : 640).

Thaillande - Enseignement professionnel et programmes de formation de niveau licence (formation continue). Ces programmes de deux ans s'organisent dans le cadre de la formation continue. L'admission à ces programmes impose l'achèvement d'un diplôme professionnel de deux ans classé dans l'enseignement supérieur de cycle court (niveau 5 de la CITE). Dès lors, le programme représente une durée cumulée totale de quatre ans d'études dans l'enseignement supérieur. Après l'achèvement complet, les sortants se voient décerner un diplôme équivalent à une licence. (CITE-P : 665 ; CITE-A : 660). 
Tunisie - (Licence appliquée). Ce programme de premier diplôme universitaire de trois ans exige l'achèvement du deuxième cycle de l'enseignement secondaire avec accès à l'enseignement supérieur comme critère minimal d'admission. Lorsqu'ils l'achèvent, les sortants reçoivent une (Licence appliquée) dans un domaine d'études particulier conduisant au marché du travail et à un niveau d'enseignementplus élevé. (CITE-P : 665 ; CITE-A : 660).

Royaume-Uni - Honours degree - Bachelor's degree programme (Programme de Diplôme de spécialisation programme de licence). II s'agit d'un premier diplôme d'enseignement, généralement décerné après trois ans d'études. Le plus souvent, les étudiants doivent satisfaire des examinateurs dans une série d'examens annuels ou par un système d'évaluation continue, et également passer un examen de diplôme final au terme du programme. Le diplôme de spécialisation implique habituellement l'étude d'un sujet principal et d'un seul sujet complémentaire. (CITE-P : 665 ; CITE-A : 660).

États-Unis - Bachelor's degree programme (Programme de licence). II s'agit le plus souvent d'un programme de quatre ans mené à bien dans des collèges ou des universités. Pour accéder à ces programmes de premier cycle universitaire, il faut généralement un diplôme de high school ou son équivalent. Les détenteurs de la licence peuvent entrer sur le marché du travail ou poursuivre leurs études dans des programmes de second cycle universitaire (Master ou PhD) ou professionnel (droit, médecine, dentisterie). (CITE-P : 665 ; CITE-A : 660)

\section{Exemples Groupe 2 :}

Programmes longs de premier diplôme de niveau licence ou équivalent (plus de quatre ans) [CITE-P : 646,656 ou 666 ; CITE-A : 640,650 ou 660]

Algérie - (Ingénieur d'état). II s'agit d'un programme de l'enseignement supérieur de cinq ans qui s'offre aux diplômés du deuxième cycle de l'enseignement secondaire. À son achèvement, les étudiants sortants de ce programme se voient décerner un diplôme d'(Ingénieur d'état) dans différents domaines d'études. (CITE-P : 666 ; CITE-A : 660).

Colombie - Título Universitario (Diplôme universitaire). Ces programmes sont des premiers diplômes universitaires de longue durée (au moins quatre ans, généralement cinq), qui permettent aux étudiants d'obtenir un diplôme professionnel ou un diplôme dans une matière spécifique. Pour être admis dans le programme, il faut avoir achevé le deuxième cycle de l'enseignement secondaire (niveau 3 de la CITE) et avoir réussi l'examen national d'entrée dans l'enseignement supérieur. (CITE-P : 666 ; CITE-A : 660).

Danemark - Mellemlange videregående uddannelser - professionsbachelor (Enseignement supérieur, cycle intermédiaire, licence professionnelle). On accède à ces programmes de cinq ans en achevant avec succès le deuxième cycle de l'enseignement secondaire (niveau 3 de la CITE). Ils préparent les étudiants à un emploi professionnel dans certains domaines comme l'ingénierie. Le diplôme est considéré comme étant d'un niveau similaire à celui d'autres licences, avec des études d'une durée de trois à quatre ans. Lors de l'achèvement du programme, les étudiants peuvent commencer à exercer la profession étudiée ou accéder à un programme de niveau master. (CITE-P : 656 ; CITE-A : 650).

Mexique - Licenciatura universitaria (Licence universitaire, cinq ans). Le critère d'admission de ces programmes universitaires est le Bachillerato (certificat d'achèvement du deuxième cycle de l'enseignement secondaire). Alors que la durée d'autres programmes Licenciatura est inférieure d'un an, les diplômes couronnant un enseignement de cinq ans sont considérés comme similaires et appartiennent tous au niveau 6 de la CITE. Lorsqu'ils achèvent ces programmes, les étudiants peuvent accéder au Maestría (programme de niveau master, CITE 7). (CITE-P : 646 ; CITE-A : 640).

République arabe syrienne - (Programmes d'ingénierie technique). Il s'agit de programmes d'enseignement supérieur de cinq ans dans le domaine de l'ingénierie dont le critère minimal d'admission est l'achèvement du deuxième cycle du secondaire. Après l'achèvement complet, les étudiants sortants se voient décerner une licence en ingénierie. (CITE-P : 666 ; CITE-A : 660).

\section{Exemples Groupe 3 :}

Deuxième diplôme ou diplôme supplémentaire (après achèvement complet d'un programme de niveau licence ou équivalent) [CITE-P : 647,657 ou 667 ; CITE-A : 640,650 ou 660 ]

Côte d'Ivoire - Enseignement universitaire général : $2^{e}$ cycle (Maîtrise). II s'agit d'un programme de deuxième diplôme qui suit l'achèvement complet d'une Licence, un programme de niveau licence. II ne dure qu'un an et n'a pas de composante de recherche élaborée. (CITE-P : 667 ; CITE-A : 660). 
Inde - Licence en enseignement. II s'agit d'un programme d'un an dans l'enseignement supérieur proposé aux détenteurs d'une licence dans différentes disciplines intéressés par une carrière dans l'enseignement. Le programme est couronné par une licence en enseignement. (CITE-P : 667 ; CITE-A : 660).

Irlande - Diplôme supérieur. Ce diplôme est normalement décerné à la suite de l'achèvement d'un programme d'un an se déroulant dans un établissement reconnu d'enseignement supérieur. Le critère d'admission dans un programme de Diplôme supérieur est généralement une licence de spécialisation, mais une licence ordinaire peut aussi suffire. Les personnes achevant avec succès un tel programme peuvent accéder à un programme de diplôme de second cycle supérieur, de master ou, dans certains cas, à des programmes conduisant à un doctorat. (CITE-P : 667 ; CITE-A : 660).

Maroc - (Programme de formation d'instituteur). II s'agit d'un programme d'un an nécessitant pour son admission l'achèvement d'un programme de licence (Diplôme de Licence d'études fondamentales (LEF)). Après avoir achevé le programme de formation d'instituteur, les étudiants se voient décerner le Certificat d'études normales d'instituteurs (CENI), qui permet à leurs détenteurs d'exercer la fonction d'enseignant qualifié dans l'enseignement primaire. (CITE-P : 667 et CITE-A : 660).

Norvège - Videreutdanning (Cours spécialisés). Ces programmes comprennent diverses spécialisations avec une orientation professionnelle, notamment pour des professions paramédicales et d'enseignement, la plus longue étant celle de sage-femme (deux ans après la certification de soins infirmiers). Ils se fondent sur une licence mais ne conduisent pas à un master. (CITE-P : 647 ; CITE-A : 640).

Arabie saoudite - (Programmes de diplôme supérieur). Ces programmes d'enseignement supérieur d'un à deux ans exigent l'achèvement d'une licence comme critère d'admission. Concernant le plus souvent le domaine de l'enseignement, ces programmes mènent à un diplôme supérieur considéré comme inférieur au niveau master à l'échelle nationale. (CITE-P : 667 et CITE-A : 660).

Suisse - Fachhochschule Nachdiplom, haute école spécialisée diplôme postgrade (Master d'études avancées). Après le premier diplôme, les universités proposent des programmes de niveau master ou des programmes de spécialisation ne menant pas à un diplôme de recherche (Master d'études avancées). Ils durent généralement un an et représentent 60 crédits ECTS. Des exemples : administration des affaires pour des ingénieurs ou spécialisation dans des aspects environnementaux pour des ingénieurs chimistes. La durée cumulée au niveau 6 de la CITE varie de 4 à 6,5 ans, en fonction du programme spécifique. La certification qu'ils décernent est considérée comme étant d'un niveau similaire à la première licence. (CITE-P : 647 ; CITE-A : 640).

\section{Exemples Groupe 4 :}

Programmes du niveau 6 de la CITE insuffisants pour un achèvement de niveau, avec donc un niveau d'éducation atteint correspondant au niveau 5 de la CITE [CITE-P : 641, 651 ou 661 ; CITE-A : 540,550 ou 560 ]

Burkina Faso - Diplôme universitaire d'études générales (DEUG). II s'agit d'un programme d'enseignement supérieur de deux ans nécessitant l'achèvement du deuxième cycle de l'enseignement secondaire (niveau 3 de la CITE). Après le DEUG, les étudiants peuvent évoluer vers le programme de Licence d'un an ou un diplôme de trois ans en ingénierie. (CITE-P : 661 ; CITE-A : 540).

Burundi - 1er cycle de l'enseignement supérieur. II s'agit d'un programme de deux ans constituant le premier cycle d'un programme de Licence. Le second cycle peut durer deux ou trois ans selon le domaine d'études. À la suite de l'achèvement complet du premier cycle, qui nécessite l'achèvement du deuxième cycle de l'enseignement secondaire avec accès à l'enseignement supérieur pour son admission, les étudiants peuvent poursuivre leurs études dans le second cycle. (CITE-P : 661 ; CITE-A : 540).

Canada - Programmes de Transfert universitaire. II s'agit de programmes d'une durée d'un ou deux ans proposés par des établissements non-universitaires dans le cadre d'accords spéciaux avec des universités où le collège organise la (les) première(s) année(s) du programme de diplôme universitaire. Les étudiants qui achèvent ces programmes dans les collèges peuvent ensuite transférer leurs crédits vers des programmes de licence universitaire. Bien que les effectifs scolarisés dans ces programmes soient classés au niveau 6 de la CITE, les étudiants qui achèvent ces programmes ne sont pas comptabilisés comme étant des diplômés du niveau 6 de la CITE. Le niveau d'éducation atteint est le niveau 5 de la CITE. (CITE-P : 641 ; CITE-A : 540). 
Norvège - Høgskolekandidat (Diplôme universitaire). Le diplôme universitaire est un programme d'enseignement supérieur de deux ans. Les étudiants doivent avoir achevé le deuxième cycle de l'enseignement secondaire (niveau 3 de la CITE) avant leur entrée. Ce diplôme devient souvent une part intégrale du programme de licence complet au moment de la fin des études. (CITE-P : 641 ; CITE-A : 540).

\section{Notes}

1. Les numéros de paragraphe constituent des références à la classification CITE 2011. Pour plus de détails, veuillez consulter le Guide du lecteur.

2. Bien qu'il n'existe pas encore de définitions internationalement convenues concernant les orientations académique et professionnelle, certains pays utilisent leurs définitions nationales et font appel à ces codes dans leurs cartographies de la CITE. 


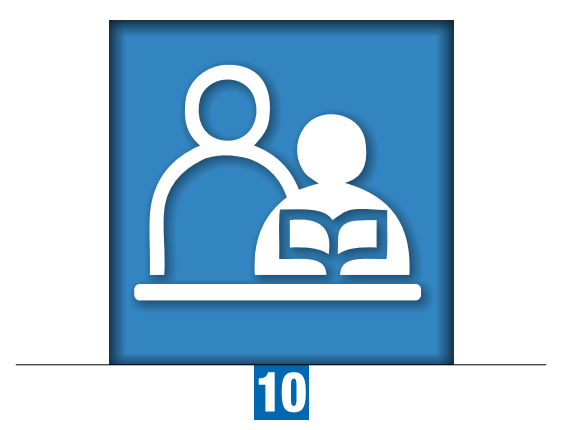

\section{Chapitre 10}

\section{Niveau 7 de la CITE 2011:}

\section{Niveau master ou équivalent}

Les programmes de niveau 7 de la CITE, ou « niveau master ou équivalent », ont un contenu nettement plus complexe que celui des programmes du niveau 6 de la CITE et sont généralement plus spécialisés. Le contenu de ces programmes du niveau 7 de la CITE est souvent destiné à enseigner aux participants des connaissances, aptitudes et compétences académiques et/ ou professionnelles conduisant à un deuxième diplôme ou une certification équivalente. Les programmes de ce niveau peuvent avoir une composante importante de recherche mais ils ne conduisent pas encore à l'obtention d'une certification de doctorat. La durée cumulée des études au niveau de l'enseignement supérieur est donc en général de cinq à huit ans ou même plus.

Les programmes classés au niveau 7 de la CITE peuvent être désignés de plusieurs manières, comme par exemple : programmes de master ou magister. En vue de la comparabilité internationale, l'expression « master ou équivalent » est utilisée pour qualifier le niveau 7 de la CITE. 


\section{DESCRIPTION}

\section{Définition}

$(\$ 241)^{1}$ Les programmes du niveau 7 de la CITE, ou « niveau master ou équivalent ", sont souvent destinés à enseigner aux participants des connaissances, aptitudes et compétences académiques et/ou professionnelles conduisant à un deuxième diplôme ou une certification équivalente. Les programmes de ce niveau peuvent avoir une composante importante de recherche mais ils ne conduisent pas encore à l'obtention d'une certification de doctorat. Les programmes de ce niveau se fondent généralement sur la théorie mais ils peuvent inclure un cursus pratique ; ils se fondent également sur des travaux de recherche de pointe et/ou les meilleures pratiques professionnelles.

(§243) Les programmes de niveau 7 de la CITE ont un contenu nettement plus complexe que celui des programmes du niveau 6 de la CITE et sont généralement plus spécialisés. À l'achèvement de ces programmes, il est possible de poursuivre au niveau 8 de la CITE (enseignement de niveau doctorat) bien que tous les programmes du niveau 7 de la CITE ne donnent pas un accès direct au niveau 8 de la CITE.

\section{Critères de classification (\$228)}

\section{Critères principaux}

a) Contenu fondé sur la théorie et/ou la pratique professionnelle

(§241) Les programmes de ce niveau se fondent généralement sur la théorie mais ils peuvent inclure un cursus pratique; ils se fondent également sur des travaux de recherche de pointe et/ou les meilleures pratiques professionnelles.

b) Position dans la structure nationale des diplômes et des certifications

(§246) Le plus souvent, les programmes de ce niveau d'enseignement préparent à un deuxième diplôme ou à un diplôme supplémentaire après un premier diplôme décerné à l'issue de programmes de niveau 6 ou 7 de la CITE.

(\$247) Les programmes d'une durée minimale de cinq ans préparant à un premier long diplôme/ certification sont inclus dans ce niveau s'ils sont équivalents aux programmes de niveau master en termes de complexité du contenu. Les études professionnelles très spécialisées d'une durée cumulée similaire ou plus longue dans l'enseignement supérieur (par exemple médecine, dentisterie, science vétérinaire et dans certains cas droit ou sciences de l'ingénieur) couvrant, du point de vue de l'étendue et de la profondeur, un contenu équivalent sont également classées dans ce niveau.

a) Critères d'admission

(\$243) L'admission aux programmes de niveau 7 de la CITE conduisant à un deuxième diplôme ou à un diplôme supplémentaire exige normalement l'achèvement complet d'un programme du niveau 6 ou 7 de la CITE. Dans le cas des programmes de longue durée préparant à un premier diplôme équivalent à un master, l'admission exige l'achèvement complet d'un programme du niveau 3 ou 4 de la CITE donnant accès à l'enseignement supérieur.

\section{Critères subsidiaires}

b) Durée cumulée minimale du programme de premier diplôme long

(§247) Les programmes d'une durée minimale de cinq ans préparant à un premier long diplôme/certification sont inclus dans ce niveau.

c) Accès direct aux programmes de niveau 8 de la CITE

(§249) Les programmes de l'enseignement supérieur qui permettent d'accéder directement au niveau 8 de la CITE sont généralement classés au niveau 7 de la CITE. Toutefois, tous les programmes du niveau 7 de la CITE ne donnent pas un accès au niveau 8 de la CITE. 


\section{Dimensions complémentaires}

(§251) Deux dimensions permettent de distinguer les programmes éducatifs du niveau 7 de la CITE.

(§252) Orientation du programme :

- Académique ; et

- Professionnel ${ }^{2}$.

(§253) Durée du programme et position dans la structure nationale des diplômes et certifications

- Cycle (ou programme) d'un premier diplôme au niveau master ou équivalent d'une durée cumulée théorique (au niveau de l'enseignement supérieur) de moins de cinq ans et donc insuffisant pour un achèvement du niveau 7 de la CITE ;

- Programme de premier diplôme long au niveau 7 de la CITE (niveau master ou équivalent), d'une durée cumulée théorique (au niveau de l'enseignement supérieur) d'au moins cinq ans (ne nécessitant pas un enseignement supérieur préalable) ;

- Programme de deuxième diplôme ou de diplôme supplémentaire, après achèvement complet d'un programme de niveau licence ou équivalent, au niveau 7 de la CITE (niveau master ou équivalent) ; et Programme de deuxième diplôme ou de diplôme supplémentaire au niveau master ou équivalent après achèvement complet d'un autre programme de niveau master ou équivalent au niveau 7 de la CITE (niveau master ou équivalent).

\section{CORRESPONDANCES ENTRE LA CITE 2011 ET LA CITE 1997}

Voir la partie correspondance dans le chapitre sur l'enseignement supérieur.

\section{DIRECTIVES POUR LA CLASSIFICATION DES PROGRAMMES ET DE LEURS CERTIFICATIONS RECONNUES CORRESPONDANTES AU NIVEAU 7 DE LA CITE}

Les programmes de niveau 7 de la CITE ont un contenu nettement plus complexe que celui des programmes du niveau 6 de la CITE et sont généralement plus spécialisés (\$243). Le contenu de ces programmes du niveau 7 de la CITE est souvent destiné à enseigner aux participants des connaissances, aptitudes et compétences académiques et/ou professionnelles conduisant à un deuxième diplôme ou une certification équivalente. Les programmes de ce niveau peuvent avoir une composante importante de recherche mais ils ne conduisent pas encore à l'obtention d'une certification de doctorat (\$241).

Ils sont traditionnellement dispensés dans des universités et des établissements d'enseignement supérieur équivalents (\$224). II est important de noter que les programmes portant un nom similaire à « licence » ne doivent être inclus dans le niveau 7 de la CITE que si la complexité de leur contenu est équivalente et s'ils répondent aux autres critères principaux (\$244).

L'admission aux programmes de niveau 7 de la CITE conduisant à un deuxième diplôme ou à un diplôme supplémentaire exige normalement l'achèvement complet d'un programme du niveau 6 ou 7 de la CITE. Dans le cas des programmes de longue durée préparant à un premier diplôme équivalent à un master, l'admission exige l'achèvement complet d'un programme du niveau 3 ou 4 de la CITE donnant accès à l'enseignement supérieur. L'admission peut dépendre du choix du sujet et/ou des notes obtenues au niveau 3 ou 4 de la CITE. II peut aussi s'avérer nécessaire de passer et de réussir des examens d'entrée (§243).

Le plus souvent, les programmes de ce niveau d'enseignement préparent à un deuxième diplôme ou à un diplôme supplémentaire (Exemples Groupe 1) après un premier diplôme décerné à l'issue de programmes de niveau 6 ou 7 de la CITE (§248). Les certifications équivalentes telles que des titres professionnels supplémentaires (Exemples Groupe 1) sont également classées au niveau 7 de la CITE, sauf si elles sont déjà classées au niveau 6 de la CITE (\$246). Les deux types de programmes durent généralement un à quatre ans d'études à plein temps. Concernant les systèmes éducatifs dans lesquels les diplômes sont accordés sur la base de cumul de crédits, une durée et une intensité comparables doivent être exigées. La durée cumulée des études au niveau de l'enseignement supérieur est donc en général de cinq à huit ans ou même plus (\$248). 
Les programmes d'une durée minimale de cinq ans préparant à un premier long diplôme/ certification sont inclus dans ce niveau s'ils sont équivalents aux programmes de niveau master en termes de complexité du contenu. Ces programmes imposent généralement la rédaction d'une thèse ou d'un mémoire élaboré (§247). (Exemples Groupe 2).

Les études professionnelles très spécialisées d'une durée cumulée similaire ou plus longue dans l'enseignement supérieur (par exemple médecine, dentisterie, science vétérinaire et dans certains cas droit ou sciences de l'ingénieur) couvrant, du point de vue de l'étendue et de la profondeur, un contenu équivalent mais ne nécessitant généralement pas la rédaction d'une thèse ou d'un mémoire sont également classées dans ce niveau (§247). (Exemples Groupe 3).

À l'achèvement de ces programmes, il est possible de poursuivre au niveau 8 de la CITE (enseignement de niveau doctorat) bien que tous les programmes du niveau 7 de la CITE ne donnent pas un accès direct au niveau 8 de la CITE (\$243).

\section{NIVEAU D'ÉDUCATION ATTEINT AU NIVEAU 7 DE LA CITE}

Le niveau d'éducation atteint au niveau 7 de la CITE exige l'achèvement complet - menant à une certification reconnue d'un programme qui représente une durée cumulée minimale d'au moins cinq ans dans l'enseignement supérieur. Dans les pays de l'Espace européen de l'enseignement supérieur, cela représente l'équivalent d'au moins 300 crédits du Système européen de transfert de crédits.

(§256) En ce qui concerne le niveau d'éducation atteint, les certifications intermédiaires reconnues obtenues lors de l'achèvement complet d'un cycle (ou d'un programme) de premier diplôme de niveau master ou équivalent considéré comme insuffisant pour être pris en compte comme achèvement du niveau 7 de la CITE sont classées dans le niveau 6 de la CITE.

L'achèvement complet de programmes représentant moins de cinq ans d'études dans l'enseignement supérieur mène normalement à un niveau d'éducation atteint correspondant au niveau 6 de la CITE. (Exemples Groupe 4)

(§257) Les certifications intermédiaires reconnues obtenues lors de l'achèvement complet d'un cycle (ou d'un programme) de niveau doctorat ou équivalent considéré comme insuffisant pour être pris en compte comme achèvement du niveau 8 de la CITE sont classées dans le niveau 7 de la CITE pour ce qui est du niveau d'éducation atteint.

Les diplômes honoris causa décernés par les universités sur la base d'autres considérations et non de travaux de recherche ne sont pas reconnus pour la détermination du niveau d'éducation atteint.

\section{EXEMPLES DE PROGRAMMES ET DE CERTIFICATIONS CLASSÉS AU NIVEAU 7 DE LA CITE}

(§244) Les programmes classés au niveau 7 de la CITE peuvent être désignés de plusieurs manières, comme par exemple : programmes de master ou magister. Toutefois, il est important de noter que les programmes portant un nom similaire à " master » ne doivent être inclus dans le niveau 7 de la CITE que s'ils répondent aux critères décrits dans le paragraphe 245 de la CITE 2011. En vue de la comparabilité internationale, l'expression « master ou équivalent » est utilisée pour qualifier le niveau 7 de la CITE.

\section{Exemples Groupe 1 :}

Programme de deuxième diplôme ou de diplôme supplémentaire au niveau master ou équivalent (après achèvement complet d'un programme de niveau licence ou équivalent) [CITE-P : 747, 757 ou 767 ; CITE-A : 740, 750 ou 760]

Australie - Master. Il s'agit d'un diplôme supérieur que l'on obtient généralement après deux années d'études suivant une licence (spécialisation). Après avoir réussi une licence, l'entrée dans un programme de Master est possible en achevant un cours d'un an de qualification au Master. Les Masters peuvent s'obtenir après avoir mené à bien des travaux 
de recherche (généralement après une période d'emploi) couronnés par la remise d'une thèse ou suivi des cours en parallèle avec un emploi professionnel. (CITE-P : 767 ; CITE-A : 760).

Colombie - Maestría (Master). Ce programme mène au titre de Magister (Master) et nécessite un titre professionnel ou un diplôme universitaire dans les domaines de la technologie, de la science, des sciences humaines, des arts ou de la philosophie pour y accéder. La durée du programme est généralement deux ans. (CITE-P : 767, CITE-A : 760).

Japon - Daigakuin Shushi katei. (Programme universitaire menant au shushi (Master)). L'achèvement du diplôme shushi impose deux années d'études à temps plein après la gakushi (licence), offrant 30 crédits et une composante importante de recherche culminant par une thèse. (CITE-P : 767 ; CITE-A : 760).

Mexique - Maestría (Programmes de Master). Ce programme implique une recherche élaborée et l'acquisition de connaissances spécialisées sur des sujets et des domaines d'études spécifiques. La durée du programme est généralement deux ans. Le critère d'admission est un programme de Licenciatura (licence) de quatre ou cinq ans. (CITE-P : 767 ; CITE-A : 760).

Sao Tomé-et-Principe - Mestrado (Programmes de Master). II s'agit d'un programme de deuxième cycle de deux ans classique menant à un programme de deuxième diplôme qui suit l'achèvement complet d'une License, un programme de niveau licence. L'achèvement de ce programme, le plus élevé dans le système éducatif national, mène à un Master (Mestrado). (CITE-P : 767 ; CITE-A : 760).

Slovénie - Magistrsko izobraževanje (2. bolonjska stopnja) (Master). Ces études durent un à deux ans, après achèvement complet du niveau 6 de la CITE ( $1^{\mathrm{er}}$ cycle de Bologne). Les étudiants sortants obtiennent un Master (avec le titre professionnel) qui donne accès aux études du niveau doctorat. (CITE-P : 767 ; CITE-A : 760).

États-Unis - Programme de premier diplôme professionnel. L'achèvement de ces programmes implique à la fois l'achèvement des exigences académiques pour commencer à exercer une profession donnée et un niveau d'aptitude professionnelle supérieur aux exigences normales pour une licence. Ces programmes durent généralement trois ans et doivent être précédés d'au moins deux ans au niveau 6 de la CITE (bien que la plupart nécessite une licence de quatre ans). Ces premiers diplômes professionnels constituent le parcours normal pour devenir un professionnel dans les domaines de la dentisterie, de la médecine, de l'optométrie, de la pharmacie, de la médecine vétérinaire, du droit et de la théologie. (CITE-P : 757 ; CITE-A : 750).

\section{Exemples Groupe 2 : \\ LProgramme de premier diplôme long au niveau master ou équivalent (au moins 5 ans) [CITE-P : 746, 756 ou 766 ; CITE-A : 740, 750 ou 760]}

Gambie - Diplôme de médecine. II s'agit d'un programme de sept ans nécessitant l'achèvement du deuxième cycle de l'enseignement secondaire. Au terme du programme, les étudiants sortants se voient décerner un Master en Médecine. (CITE-P : 766 ; CITE-A : 750).

Allemagne - Universitäten - Diplom, Magister. (Université - Magister) Ces anciens programmes universitaires n'ont pas été adaptés dans la structure de Bologne. Après 8 à 10 semestres, ils menaient à un premier diplôme équivalent à un Master (CITE-P : 746 ; CITE-A : 740). Ces programmes imposaient la rédaction d'une thèse ou d'un mémoire élaboré accompagnée de cours dans les premiers semestres du programme. Les diplômés du programme pouvaient accéder à des programmes de doctorat. (CITE-P : 746 ; CITE-A : 740).

Allemagne - Universitäten - Staatsexamen (Programmes universitaires dans les domaines de la formation d'enseignants, de la dentisterie, de la médecine, de la médecine vétérinaire, de la pharmacie, du droit et de la théologie). Après 8 à 10 semestres d'études universitaires, ces programmes mènent à un examen national équivalent à un Master (niveau 7 de la CITE). (CITE-P : 746 ; CITE-A : 740).

Japon - Daigaku Yakugaku Ishigaku Juigaku (Programme universitaire de premier cycle). Le gakushi est la première certification décernée après six ans d'études de pharmacie (cours pratiques uniquement), de médecine, de dentisterie et de médecine vétérinaire. Outre les études dans un de ces domaines spécialisés, un enseignement général (incluant les sciences humaines, sociales et naturelles) est obligatoire pour tous les étudiants. (CITE-P : 766 ; CITE-A : 760). 
Kyrgyzstan - Высшее профессиональное образование, ведущее к поступлению в программы продвинутых научных исследований и получению диплома специалиста (Programme de premier diplôme universitaire long, menant à un diplôme de spécialiste). Ce premier diplôme universitaire a une durée de cinq ans. Le critère d'admission minimal est le certificat d'achèvement du deuxième cycle de l'enseignement secondaire. Le programme comprend des cours au contenu nettement plus complexe que d'autres programmes communs de la CITE 6. À la fin du programme, les diplômés reçoivent un diplôme de spécialiste. (CITE-P : 766 ; CITE-A : 760).

République démocratique populaire lao - Licence de médecine. Ce programme universitaire de premier diplôme long (six ans) exige l'achèvement du deuxième cycle de l'enseignement secondaire avec accès à l'enseignement supérieur (Certificat de Baccalauréat) comme critère d'admission. II s'agit d'un programme hautement spécialisé de très longue durée. Bien que le titre du diplôme obtenu soit une Licence en médecine, il implique des études nettement plus complexes qu'un programme de licence normal et doit donc être classé au niveau Master. (CITE-P : 766 ; CITE-A : 760 ).

Mozambique - Mestrado Integrado (Master intégré). Ce programme de premier diplôme de cinq ans exige l'achèvement du deuxième cycle de l'enseignement secondaire comme critère d'admission minimal. Au terme du programme, les étudiants sortants se voient décerner un Master. (CITE-P : 766 ; CITE-A : 760).

Portugal - Ensino Superior - Licenciatura (Enseignement supérieur - premier diplôme long, quatre à six ans). Ce sont des programmes qui ont précédé Bologne. Ces programmes d'une durée de quatre à six ans, généralement proposés dans les universités, étaient accessibles après un achèvement complet de l'enseignement secondaire. Dans la plupart des domaines d'études, les programmes duraient quatre à cinq ans. II existait aussi des programmes de six ans. Les étudiants terminant le programme avec une note moyenne de 16 ou plus pouvaient accéder directement au niveau doctorat. Les certifications finales obtenues à l'issue de ces programmes pré-Bologne sont reconnues à l'échelle nationale comme équivalant à la CITE 7 dans la CITE 2011. (CITE-P : 766 ; CITE-A : 760).

\section{Exemples Groupe 3 :}

Programme de deuxième diplôme ou de diplôme supplémentaire au niveau master ou équivalent (après un programme de master ou équivalent) [CITE-P : 748, 758, 768 ; CITE-A : 740, 750, 760]

Australie - Docteur en Philosophie par des cours. Ces programmes de trois ans sont accessibles après un achèvement complet d'un premier master. Ils offrent une spécialisation accrue dans des domaines d'étude professionnels et académiques. Le programme se fonde sur des cours et ne mène pas à une thèse de niveau doctorat sur un projet de recherche original représentant une contribution significative à la connaissance dans le domaine d'étude. C'est la raison pour laquelle le diplôme obtenu est considéré comme similaire à celui d'un niveau master (niveau 7 de la CITE). (CITE-P : 748 ; CITE-A : 740).

Canada - Programme qualifiant de troisième cycle. Pour accéder à ces programmes d'une durée de un à deux ans, il faut achever un premier master avec succès. Ces programmes préparent les étudiants à poursuivre leurs études au niveau doctorat si l'enseignement qu'ils avaient suivi précédemment ne satisfaisait pas toutes les exigences requises (par exemple, les étudiants ont étudié une matière mais souhaitent poursuivre leurs études au niveau doctorat dans un domaine différent ou plus spécialisé ; ou leur premier master est plus professionnel qu'académique). La certification obtenue est considérée comme appartenant au niveau master (niveau 7 de la CITE). Lorsqu'ils achèvent le programme, les étudiants peuvent poser leur candidature pour accéder à un programme de doctorat. (CITE-P : 768 ; CITE-A : 760 ).

Allemagne - Universitäten - zweiter Master (Études Universitaires - deuxième master). Pour accéder à ces programmes d'une durée de un à deux ans, il faut achever un premier master avec succès. Ils offrent une spécialisation accrue dans des domaines d'étude professionnels et académiques. Le diplôme obtenu est toujours considéré comme similaire à celui d'un niveau master (niveau 7 de la CITE). Lorsqu'ils achèvent le programme, les étudiants peuvent accéder à des études de niveau doctorat (Promotionsstudium). (CITE-P : 748 ; CITE-A : 740).

Italie - Master di $2^{\circ}$ livello (Master de deuxième niveau). Ces programmes d'un an sont accessibles à la suite de l'achèvement complet d'un Master di $1^{\circ}$ livello (master de premier niveau d'une durée d'un an) et offrent une spécialisation accrue dans un domaine d'étude donné. Le diplôme obtenu est toujours considéré comme appartenant au niveau master (niveau 7 de la CITE). Lorsqu'ils achèvent le programme, les étudiants peuvent accéder à un programme de recherche de niveau doctorat (Dottorato di ricerca). (CITE-P : 768 ; CITE-A : 760). 
Kazakhstan - Резидентура, послевузовское углублённое медицинское образование (Résidence en médecine, enseignement médical approfondi de deuxième cycle). Ces programmes de deuxième cycle ont une durée qui varie de deux à quatre ans en fonction de la spécialité médicale. Pour accéder à ces programmes, il faut achever un premier master avec succès (une combinaison de diplôme de médecine générale et d'un internat). Les programmes, qui incluent des composantes de recherche et de cours en plus d'une résidence à l'hôpital, conduisent à un certificat d'achèvement d'études médicales (Свидетельство об окончании резидентуры). (CITE-P : 768, CITE-A : 760).

Mauritanie - Formation des enseignants au secondaire. Le critère d'admission minimal pour ce programme d'un an est un master. Au terme du programme, les participants reçoivent le Certificat d'aptitude au professorat de l'enseignement secondaire (CAPES), qui permet à ses détenteurs d'exercer en tant qu'enseignants qualifiés dans l'enseignement secondaire. (CITE-P : 768, CITE-A : 760).

Népal - Master en Philosophie. II s'agit d'un programme de deux ans nécessitant l'achèvement d'un master pour y accéder. À la fin du programme, les participants reçoivent un Master en Philosophie. (CITE-P : 768 ; CITE-A : 760).

Espagne - Especialidades Sanitarias (Études de soins de santé post-diplôme). Ces programmes sont accessibles après un achèvement complet d'un premier master et permettent une spécialisation dans des domaines liés aux études de soins de santé. Les programmes ont une durée de deux à cinq ans selon le sujet. Le diplôme obtenu (Título de Especialista Sanitariois) est toujours considéré comme appartenant au niveau master (niveau 7 de la CITE). Ces programmes donnent généralement accès aux programmes de niveau doctorat. (CITE-P : 758 ; CITE-A : 750).

Tunisie - (Diplôme national de Révision comptable). II s'agit d'un programme d'un an qui succède à un programme de deuxième diplôme de deux ans de niveau master en Audit (Révision comptable). Au terme du programme, les participants reçoivent le Diplôme national de la révision comptable. (CITE-P : 768, CITE-A : 760).

\section{Exemples Groupe 4 :}

Cycle (ou programme) d'un premier diplôme au niveau master ou équivalent insuffisant pour un achèvement du niveau 7 de la CITE (durée cumulée théorique au niveau de l'enseignement supérieur de moins de cinq ans) et menant donc à un niveau d'éducation atteint au niveau 5 ou 6 de la CITE [CITE-P : 741, 751 ou 761 ; CITE-A : 540, 550, 640, 650 ou 660]

Tunisie - (Cycle préparatoire). Ce programme est considéré comme le premier cycle du programme de diplôme d'ingénierie national, classé comme un premier diplôme long de niveau master. Seuls les meilleurs diplômés du deuxième cycle du secondaire, théoriquement à partir de 19 ans, peuvent bénéficier de l'accès à ce programme de deux ans. Au terme du programme, les participants se voient décerner le diplôme national du cycle préparatoire, qui ne sert qu'à poursuivre le programme de diplôme d'ingénierie national. (CITE-P : 761 ; CITE-A : 544).

\section{Notes}

1. Les numéros de paragraphe constituent des références à la classification CITE 2011. Pour plus de détails, veuillez consulter le Guide du lecteur.

2. Bien qu'il n'existe pas encore de définitions internationalement convenues concernant les orientations académique et professionnelle, certains pays utilisent leurs définitions nationales et font appel à ces codes dans leurs cartographies de la CITE. 



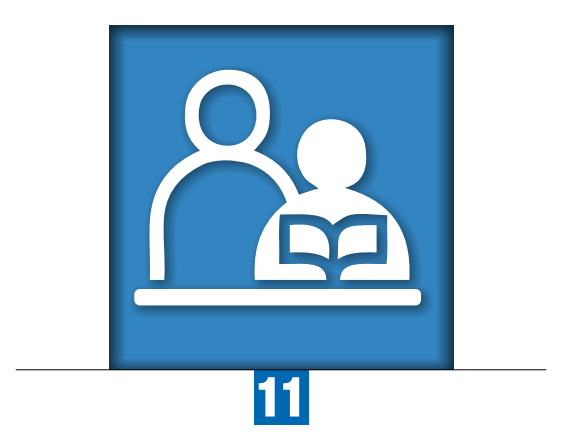

\section{Chapitre 11}

\section{Niveau 8 de la CITE 2011:}

\section{Niveau doctorat ou équivalent}

Les programmes du niveau 8 de la CITE, ou « niveau doctorat ou équivalent », sont principalement destinés à l'obtention d'une certification de chercheur hautement qualifié. Les programmes de ce niveau de la CITE sont consacrés à des études approfondies et à des travaux de recherche originaux et sont dispensés presque exclusivement par des établissements d'enseignement supérieur orientés vers la recherche, comme des universités par exemple. Les programmes de doctorat existent aussi bien dans des domaines académiques que professionnels.

La durée théorique de ces programmes est trois années d'études équivalent temps plein dans la plupart des pays, mais le temps réel nécessaire pour achever les programmes est généralement plus long.

Les programmes classés au niveau 8 de la CITE peuvent être désignés de plusieurs manières, comme par exemple : PhD, DPhil, D.Lit, D.Sc, LL.D, Doctorat, etc. En vue de la comparabilité internationale, l'expression " niveau doctorat ou équivalent » est utilisée pour qualifier le niveau 8 de la CITE. 


\section{DESCRIPTION}

\section{Définition}

$(\$ 259)^{1}$ Les programmes du niveau 8 de la CITE, ou " niveau doctorat ou équivalent ", sont principalement destinés à l'obtention d'une certification de chercheur hautement qualifié. Les programmes de ce niveau de la CITE sont consacrés à des études approfondies et à des travaux de recherche originaux et sont dispensés presque exclusivement par des établissements d'enseignement supérieur orientés vers la recherche, comme des universités par exemple. Les programmes de doctorat existent aussi bien dans des domaines académiques que professionnels.

(\$261) L'accès aux programmes de niveau 8 de la CITE ou aux postes d'assistants de recherche exige normalement l'achèvement complet de programmes spécifiques de niveau 7 de la CITE. Le niveau 8 de la CITE exige au minimum trois années d'études équivalent temps plein, pour une durée cumulée totale d'au moins sept ans à temps plein dans l'enseignement supérieur.

(§260) Le niveau 8 de la CITE s'achève normalement par la remise et la soutenance d'une thèse ou d'un travail écrit équivalent d'une qualité suffisante pour en permettre la publication et qui doit représenter une contribution appréciable aux connaissances dans le domaine d'études. Ces programmes se fondent donc le plus souvent sur des travaux de recherche et non uniquement sur des cours.

\section{Critères de classification}

\section{Critères principaux}

a) Exigence de travaux écrits

(§260) L'achèvement complet d'un programme de niveau 8 de la CITE exige la remise d'une thèse ou d'un travail écrit équivalent d'une qualité suffisante pour en permettre la publication, qui représente le produit de travaux de recherche originaux de même qu'une contribution appréciable aux connaissances dans le domaine d'études.

b) Critères d'admission

(§261) L'accès aux programmes de niveau 8 de la CITE ou aux postes d'assistants de recherche exige normalement l'achèvement complet de programmes spécifiques de niveau 7 de la CITE.

c) Durée minimale du niveau

(§265) Le niveau 8 de la CITE exige au minimum trois années d'études équivalent temps plein.

\section{Critères subsidiaires}

a) Diplôme/certification doctoral exigé pour des professions spécifiques

(§266) L'obtention d'une certification de niveau 8 de la CITE constitue souvent une condition à remplir afin d'accéder à des postes d'enseignants dans des établissements éducatifs qui proposent des programmes de niveaux 6,7 et 8 de la CITE, ainsi qu'à des postes de chercheurs pour les gouvernements et les entreprises.

\section{Dimensions complémentaires}

(§268) Une dimension peut être utilisée pour distinguer les programmes éducatifs du niveau 8 de la CITE :

(§269) Orientation du programme

- Académique ; et

- Professionnel2. 


\section{CORRESPONDANCES ENTRE LA CITE 2011 ET LA CITE 1997}

Voir la partie correspondance dans le chapitre sur l'enseignement supérieur.

\section{DIRECTIVES POUR LA CLASSIFICATION DES PROGRAMMES ET DE LEURS CERTIFICATIONS RECONNUES CORRESPONDANTES AU NIVEAU 8 DE LA CITE}

Ce niveau est réservé aux programmes d'enseignement supérieur conduisant directement à l'obtention d'une certification de chercheur hautement qualifié. La durée théorique de ces programmes est trois années d'études équivalent temps plein dans la plupart des pays (pour une durée cumulée totale d'au moins sept ans d'enseignement équivalent temps plein (ETP) au niveau de l'enseignement supérieur), mais le temps réel nécessaire pour achever les programmes est généralement plus long. Les programmes sont consacrés à des études approfondies et à des travaux de recherche originaux.

II est important de noter que les programmes portant un nom similaire à " doctorat » ne doivent être inclus dans le niveau 8 de la CITE que si la complexité de leur contenu est équivalente à des programmes déjà classés à ce niveau et s'ils répondent aux autres critères principaux (\$262).

Ces programmes se fondent le plus souvent sur des travaux de recherche et non uniquement sur des cours. Dans certains systèmes éducatifs, les programmes de niveau 8 de la CITE incluent très peu ou pas de cours, et les doctorants conduisent des travaux de recherche seuls ou en petits groupes avec des degrés de supervision variables. Dans certains systèmes éducatifs, ce sont les individus employés par l'université en tant que chercheurs juniors ou assistants de recherche qui s'inscrivent comme étudiants et entreprennent ces travaux de recherche de niveau doctorat (\$260).

Bien que la plupart des pays n'aient qu'une 'première' certification de chercheur hautement qualifié (Exemples Groupe 1), certains pays décernent une certification 'intermédiaire' de chercheur hautement qualifié avant le doctorat (Exemples Groupe 2). Un nombre restreint de pays ont une 'seconde' certification de chercheur hautement qualifié (Exemples Groupe 3). II est important de rendre compte de ces certifications intermédiaires et secondes dans la structure de classification pour définir la limite entre la première certification de chercheur hautement qualifié et les autres certifications au niveau 8 de la CITE.

Les programmes exigeant un master pour y accéder et conduisant à des certifications intermédiaires de chercheur doivent être classés soit comme le premier cycle ou une composante de programmes du niveau 8 de la CITE (où l'achèvement de cette composante ne correspond pas à un achèvement du niveau 8 de la CITE) ou comme des programmes de deuxième diplôme ou diplôme supplémentaire du niveau 7 de la CITE succédant à un master ou équivalent. Cette décision d'affectation doit se fonder sur la mesure dans laquelle le programme est conçu pour conduire directement à l'obtention d'une certification de chercheur hautement qualifié. II peut également être utile de vérifier si les étudiants peuvent transférer des crédits de ce programme à un programme de doctorat, ou si la certification intermédiaire constitue une exigence pour accéder à un programme de doctorat. Les programmes principalement destinés à préparer les étudiants à une entrée directe sur le marché du travail avec des aptitudes de chercheur fondamentales ou intermédiaires doivent être classés au niveau 7 de la CITE, même si ces programmes permettent aussi aux étudiants de poursuivre leurs études en vue d'obtenir une certification de chercheur hautement qualifié.

\section{NIVEAU D'ÉDUCATION ATTEINT AU NIVEAU 8 DE LA CITE}

(\$272) En ce qui concerne le niveau d'éducation atteint, les certifications intermédiaires reconnues obtenues lors de l'achèvement complet d'un cycle (ou d'un programme) de premier diplôme de niveau doctorat ou équivalent considéré comme insuffisant pour être pris en compte comme achèvement du niveau 8 de la CITE sont classées dans le niveau 7 de la CITE.

Les doctorats honoris causa décernés par les universités sur la base d'autres considérations et non de travaux de recherche ne sont pas reconnus pour la détermination du niveau d'éducation atteint. 


\section{EXEMPLES DE PROGRAMMES ET DE CERTIFICATIONS CLASSÉS AU NIVEAU 8 DE LA CITE}

(§262) Les programmes classés au niveau 8 de la CITE peuvent être désignés de plusieurs manières, comme par exemple : PhD, DPhil, D.Lit, D.Sc, LL.D, Doctorat, etc. Toutefois, il est important de noter que les programmes portant un nom similaire à « doctorat » ne doivent être inclus dans le niveau 8 de la CITE que s'ils répondent aux critères décrits dans le paragraphe 263 de la CITE 2011. En vue de la comparabilité internationale, l'expression " niveau doctorat ou équivalent » est utilisée pour qualifier le niveau 8 de la CITE.

\section{Exemples Groupe 1 :}

Programmes de niveau doctorat ou équivalent suffisants pour l'achèvement du niveau 8 de la CITE [CITE-P : 844, 854 ou 864 ; CITE-A : 840, 850 ou 860]

Australie - Diplôme de docteur ou doctorat. On accède à ces programmes à la suite d'une licence (haute distinction) ou un master et ils comprennent généralement trois ans d'études à temps plein consacrées à la préparation d'une thèse élaborée fondée sur un projet de recherche original ayant pour résultat une contribution appréciable aux connaissances, à la compréhension et/ou l'application de connaissances dans le domaine d'étude. (CITE-P : 864 ; CITE-A : 860).

Bahrein - (Programmes de doctorat). Ces programmes nécessitent au moins l'achèvement d'un master pour y accéder. La durée de ces programmes est généralement trois à cinq ans. À la fin du programme, les participants reçoivent un Doctorat. (CITE-P : 864 ; CITE-A : 860).

Colombie - Doctorado (Doctorat). II s'agit d'un programme de niveau doctorat qui a normalement une durée de cinq ans. Le critère d'admission minimal à ce programme ne doit pas nécessairement inclure un master mais au moins un titre professionnel ou un diplôme universitaire dans les domaines de la technologie, de la science, des sciences humaines, des arts ou de la philosophie pour y accéder. (CITE-P : 864 ; CITE-A : 860).

France - Doctorat. Le Doctorat est un programme d'étude de trois ans succédant à un master et représentant huit années d'études cumulées dans l'enseignement supérieur dans des domaines tels que les sciences humaines, les sciences, les sciences économiques et le droit. Le diplôme est décerné après la remise d'une thèse fondée sur un projet de recherche original acceptable pour le responsable de l'école doctorale ou le Conseil Scientifique de l'université. Les candidats mènent des travaux de recherche personnelle constituant une contribution originale au domaine d'étude qu'ils choisissent. (CITE-P : 864 ; CITE-A : 860).

Allemagne - Promotionstudium. (Études de doctorat). Les programmes ont une durée de trois à cinq ans. Dans la plupart des cas, les étudiants doivent avoir achevé avec succès des programmes universitaires (dans des disciplines académiques) de cinq à sept ans avant d'accéder à des programmes de Promotion. Les étudiants se voient décerner un doctorat sur la base d'une thèse et d'un examen oral. Un Promotionsstudium peut être mené à bien sous la forme d'une freie Promotion (doctorat individuel supervisé), le principal modèle en Allemagne, ou sous la forme d'une strukturierte Promotion incluant des études au Graduiertenkollegs (collèges supérieurs) ou Graduiertenschulen (écoles supérieures). (CITE-P : 844 ; CITE-A : 840).

Italie - Dottorati di ricerca. (Doctorat de recherche). Ce diplôme est le diplôme académique le plus élevé qui puisse être décerné. II l'est après un minimum de trois ans passés dans un département universitaire à effectuer un programme de recherche spécifique sous la direction de professeurs universitaires. L'admission au Dottorati di ricerca est limitée et se fonde sur un examen sélectif parmi les détenteurs du Laurea magistrale (Master). (CITE-P : 864 ; CITE-A : 860).

Japon - Daigakuin Hakushi Katei (Cours de Doctorat). Le hakushi (diplôme de doctorat) est le diplôme le plus élevé décerné à des étudiants ayant achevé un cours de doctorat dans une école de deuxième cycle ou dont il a été reconnu qu'ils détenaient des certifications équivalentes. Pour achever un cours de doctorat, il faut plus de cinq ans d'études dans une école de deuxième cycle (en plus de quatre ans d'études de premier cycle), avec 30 crédits ou plus, la remise d'une thèse et la réussite d'un examen final. Les personnes ayant achevé des travaux de chercheur hautement qualifié peuvent se voir décerner le hakushi après trois ans d'études dans un établissement de deuxième cycle. (CITE-P : 864 ; CITE-A : 860). 
États-Unis - Docteur en philosophie (PhD). Le PhD est le diplôme académique le plus élevé et exige une maîtrise dans un domaine de connaissance et une capacité démontrée à mener à bien des travaux de recherche savante (trois à cinq ans habituellement au-delà du master - ce qui représente huit à dix ans d'études cumulées dans l'enseignement supérieur). (CITE-P : 844 ou 854 ; CITE-A : 840 ou 850).

\section{Exemples Groupe 2 :}

Programmes de niveau doctorat ou équivalent insuffisants pour l'achèvement du niveau 8 de la CITE, menant à un niveau d'éducation atteint au niveau 7 de la CITE [CITE-P : 841, 851 ou 861 ; CITE-A : 740, 750 ou 760 ]

Portugal - Ensino Superior: Curso de Especialização - Curso de doutoramento (Enseignement supérieur : Cours de spécialisation - cours de doctorat). Le Curso de Especialização - Curso de doutoramento constitue la première partie du Doutoramento - $3 .^{\circ}$ Ciclo (troisième cycle de l'enseignement supérieur). II consiste en unités de programmes dans une formation de recherche. Le diplôme décerné (certification Especialização) ne donne pas droit à un PhD. Le programme est de niveau doctorat mais ne mène pas à l'achèvement du doctorat. (CITE-P : 861 ; CITE-A : 760).

\section{Exemples Groupe 3 :}

Seconde certification de chercheur hautement qualifié au niveau 8 de la CITE (à la suite d'un programme de doctorat) [CITE-P : 844, 854 ou 864 ; CITE-A : 840, 850 ou 860]

Kyrgyzstan - Послевузовское профессиональное образование, степень доктора наук (Enseignement de deuxième cycle, programme Doktor nauk). II s'agit d'une seconde certification de chercheur hautement qualifié succédant au послевузовское профессиональное образование, степень кандидата наук (Enseignement de deuxième cycle, programme kandidat nauk) menant au diplôme kandidat nauk, déjà classé dans la CITE 8. En général, le programme doktor nauk n'a pas de composante de cours et n'exige pas nécessairement une supervision formelle. Habituellement, il exige au moins deux ans de recherche. À la fin du programme, les participants reçoivent le diplôme doctor nauk (диплом с присвоением ученой степени доктора наук). (CITE-P : 864 ; CITE-A : 860).

\section{Notes}

1. Les numéros de paragraphe constituent des références à la classification CITE 2011. Pour plus de détails, veuillez consulter le Guide du lecteur.

2. Bien qu'il n'existe pas encore de définitions internationalement convenues concernant les orientations académique et professionnelle, certains pays utilisent leurs définitions nationales et font appel à ces codes dans leurs cartographies de la CITE. 



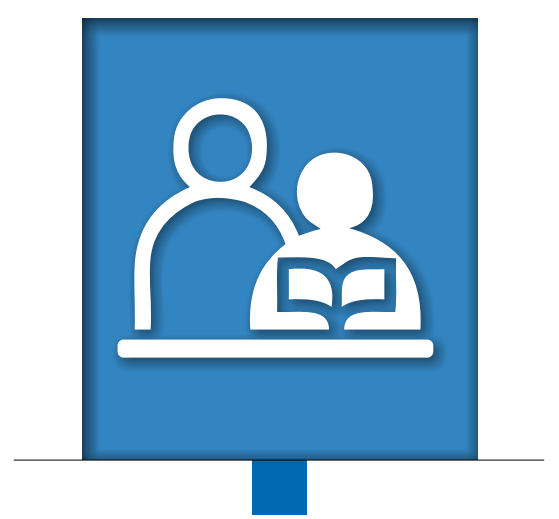

\section{Annexe A}

\section{Tableau récapitulatif des codes et des critères de la CITE 2011}

Les unités de la classification CITE sont des programmes éducatifs et les certifications reconnues correspondantes.

http://www.uis.unesco.org/Education/Pages/international-standardclassification-of-education.aspx

La classification CITE utilise 3 chiffres : le premier est le niveau éducatif, le deuxième et le troisième sont des dimensions complémentaires.

Le niveau CITE d'un programme éducatif reflète le degré de complexité et de spécialisation du contenu du programme mesuré en fonction de gradations d'expériences d'apprentissage et de connaissances, d'aptitudes et de compétences que le programme est supposé enseigner. Le niveau d'éducation atteint est mesuré en fonction du niveau d'enseignement le plus élevé qu'un individu a achevé complètement, qui est normalement certifié par une certification reconnue. Si le niveau d'enseignement le plus élevé n'est pas achevé avec succès, le niveau d'éducation atteint est celui atteint avant l'entrée dans le programme. 


\section{NIVEAUX ET DIMENSIONS COMPLÉMENTAIRES DE LA CLASSIFICATION INTERNATIONALE TYPE DE L'ÉDUCATION (CITE) 2011}

\section{Codification des programmes éducatifs et du niveau d'éducation atteint}

\begin{tabular}{|c|c|c|c|}
\hline \multirow{2}{*}{\multicolumn{2}{|c|}{$\begin{array}{l}\text { Niveau } \\
1 \text { er chiffre }\end{array}$}} & \multicolumn{2}{|c|}{ Critères de classification des programmes nationaux par niveaux } \\
\hline & & Critères principaux & Critères subsidiaires \\
\hline \multicolumn{2}{|c|}{ Pas d'éducation } & - & - \\
\hline \multirow{4}{*}{0} & \multirow{4}{*}{$\begin{array}{l}\text { Éducation de la } \\
\text { petite enfance }\end{array}$} & $\begin{array}{l}\text { Apprentissage stimulé par l'environnement } \\
(\S 105)^{\star} \text { ou en interaction avec des éducateurs } \\
(\S 106) \text {. }\end{array}$ & $\begin{array}{l}\text { Qualifications du personnel : Certifications } \\
\text { pédagogiques des éducateurs ( } \$ 111) \text {. }\end{array}$ \\
\hline & & $\begin{array}{l}\text { Établissement : fondé sur l'école ou fondé sur } \\
\text { un centre }(\$ 107)\end{array}$ & Existence d'un cadre réglementaire ( $\$ 112)$. \\
\hline & & $\begin{array}{l}\text { Admission/âge : } 3 \text { ans et plus pour } \\
\text { l'enseignement préprimaire ( } \$ 102 / 108) \text {. }\end{array}$ & Généralement non obligatoire $(\S 113)$. \\
\hline & & $\begin{array}{l}\text { Intensité : } 2 \text { heures d'enseignement par jour } \\
\text { et } 100 \text { jours par an }(\$ 110) .\end{array}$ & \\
\hline \multirow{3}{*}{1} & \multirow{3}{*}{$\begin{array}{l}\text { Enseignement } \\
\text { primaire }\end{array}$} & $\begin{array}{l}\text { Enseignement avec enseignement et } \\
\text { apprentissage systématiques de la lecture, de } \\
\text { l'écriture et des mathématiques ( } \$ 125) \text {. }\end{array}$ & $\begin{array}{l}\text { Coïncide souvent avec le début de la scolarité } \\
\text { obligatoire }(\S 127) \text {. }\end{array}$ \\
\hline & & $\begin{array}{l}\text { Admission/âge et durée : âge officiel d'entrée } \\
\text { entre } 5 \text { et } 7 \text { ans ; durée typique } 6 \text { ans (éventail } \\
\text { de } 4 \text { à } 7 \text { ans) (§122). }\end{array}$ & \\
\hline & & $\begin{array}{l}\text { Enseignant : généralement un enseignant } \\
\text { principal pour un groupe }(\S 126) .\end{array}$ & \\
\hline \multirow{3}{*}{2} & \multirow{3}{*}{$\begin{array}{l}\text { Premier cycle de } \\
\text { l'enseignement } \\
\text { secondaire }\end{array}$} & $\begin{array}{l}\text { Transition vers un enseignement plus orienté } \\
\text { vers les matières }(\$ 144) \text {. }\end{array}$ & $\begin{array}{l}\text { Âge normal d'entrée entre } 10 \text { et } 13 \text { ans, le } \\
\text { plus commun étant } 12 \text { ans ( } \$ 141) .\end{array}$ \\
\hline & & $\begin{array}{l}\text { Critère d'admission : achèvement de } \\
\text { l'enseignement primaire (ou la capacité } \\
\text { d'étudier au niveau } 2 \text { de la CITE) (\$145). }\end{array}$ & $\begin{array}{l}\text { Enseignants spécialisés, avec des } \\
\text { certifications dans des matières spécifiques } \\
\text { et en pédagogie ( } \$ 147) \text {. }\end{array}$ \\
\hline & & $\begin{array}{l}\text { Durée cumulée : se termine après } 8 \text { à } 11 \text { ans } \\
\text { d'enseignement (souvent } 9 \text { ) depuis le début } \\
\text { de l'enseignement primaire ( } \$ 146) \text {. }\end{array}$ & $\begin{array}{l}\text { La fin du niveau coïncide souvent avec la fin } \\
\text { de la scolarité obligatoire ( } \$ 148) \text {. }\end{array}$ \\
\hline
\end{tabular}

\section{Notes}

* Les numéros de paragraphe constituent des références à la classification CITE 2011. Pour plus de détails, veuillez consulter le Guide du lecteur.

** Variable HATLEVEL / HATVOC de l'enquête de I'Union européenne sur la population active (Règlement de la Commission européenne 317/2013). 


\begin{tabular}{|c|c|c|c|c|c|c|}
\hline \multicolumn{4}{|c|}{ Dimensions complémentaires } & \multicolumn{3}{|l|}{ Codification } \\
\hline \multicolumn{2}{|c|}{$2^{\ominus}$ chiffre } & \multicolumn{2}{|c|}{$3^{e}$ chiffre } & $\begin{array}{l}\text { Programmes } \\
\text { éducatifís } \\
\text { CITE-P (Annexe } \\
\text { II de la CITE } \\
\text { 2011) }\end{array}$ & $\begin{array}{l}\text { Niveau } \\
\text { d'éducation } \\
\text { atteint CITE-A } \\
\text { (Annexe III de } \\
\text { la CITE 2011) }\end{array}$ & $\begin{array}{l}\text { Variable } \\
\text { HATLEVEL/ } \\
\text { HATVOC** } \\
\text { de l'enquête } \\
\text { de l'Union } \\
\text { européenne sur } \\
\text { la population } \\
\text { active }\end{array}$ \\
\hline \multicolumn{2}{|r|}{-} & \multicolumn{2}{|r|}{-} & - & 010 & 000 \\
\hline \multicolumn{7}{|c|}{ Type d'enseignement : } \\
\hline 1 & $\begin{array}{l}\text { Développement } \\
\text { éducatif de la petite } \\
\text { enfance } \\
\text { (0 à } 2 \text { ans) }\end{array}$ & - & - & 010 & \multirow[b]{2}{*}{020} & - \\
\hline 2 & $\begin{array}{l}\text { Enseignement } \\
\text { préprimaire (de } \\
3 \text { ans à l'âge de } \\
\text { l'enseignement } \\
\text { primaire) }\end{array}$ & - & - & 020 & & 000 \\
\hline- & - & - & - & 100 & 100 & 100 \\
\hline \multicolumn{2}{|c|}{$\begin{array}{l}\text { Orientation du } \\
\text { programme : }\end{array}$} & \multicolumn{2}{|c|}{$\begin{array}{l}\text { Achèvement de niveau et accès au niveau } \\
\text { supérieur de la CITE : }\end{array}$} & & & \\
\hline \multirow[t]{2}{*}{4} & \multirow[t]{2}{*}{ Général } & 1 & $\begin{array}{l}\text { Insuffisant pour un achèvement } \\
\text { complet ou partiel du niveau (durée } \\
<2 \text { ans ou durée cumulée }<8 \text { ans } \\
\text { depuis le début du niveau } 1 \text { de la } \\
\text { CITE). }\end{array}$ & 241,251 & 100 & 100 \\
\hline & & 2 & $\begin{array}{l}\text { Achèvement partiel d'un niveau } \\
\text { (programme intermédiaire avec durée } \\
\geq 2 \text { ans et durée cumulée } \geq 8 \text { ans). }\end{array}$ & 242,252 & 242,252 & 200 \\
\hline \multirow{2}{*}{5} & \multirow{2}{*}{ Professionnel } & 3 & $\begin{array}{l}\text { Achèvement d'un niveau sans accès } \\
\text { direct à la CITE } 3 \text { (durée } \geq 2 \text { ans, durée } \\
\text { cumulée } \geq 8 \text { ans). }\end{array}$ & 243,253 & 243,253 & 200 \\
\hline & & 4 & $\begin{array}{l}\text { Achèvement d'un niveau avec accès } \\
\text { direct à la CITE } 3 \text { (durée } \geq 2 \text { ans, durée } \\
\text { cumulée } \geq 8 \text { ans). }\end{array}$ & 244,254 & 244,254 & 200 \\
\hline
\end{tabular}

\section{Notes}

* Les numéros de paragraphe constituent des références à la classification CITE 2011. Pour plus de détails, veuillez consulter le Guide du lecteur.

** Variable HATLEVEL / HATVOC de l'enquête de l'Union européenne sur la population active (Règlement de la Commission européenne 317/2013). 


\begin{tabular}{|c|c|c|c|}
\hline \multirow{2}{*}{\multicolumn{2}{|c|}{$\begin{array}{l}\text { Niveau } \\
1^{\text {er }} \text { chiffre }\end{array}$}} & \multicolumn{2}{|c|}{ Critères de classification des programmes nationaux par niveaux } \\
\hline & & Critères principaux & Critères subsidiaires \\
\hline \multirow{3}{*}{3} & \multirow{3}{*}{$\begin{array}{l}\text { Deuxième cycle } \\
\text { de l'enseignement } \\
\text { secondaire }\end{array}$} & $\begin{array}{l}\text { Deuxième/dernier cycle de l'enseignement } \\
\text { secondaire, sous la forme de programmes } \\
\text { d'enseignement général ou professionnel } \\
(\S 167) \text {. }\end{array}$ & $\begin{array}{l}\text { Programmes plus différenciés : éventail élargi } \\
\text { d'options et de filières (§169). }\end{array}$ \\
\hline & & $\begin{array}{l}\text { Critères d'admission : achèvement du premier } \\
\text { cycle de l'enseignement secondaire (ou la } \\
\text { capacité d'étudier au niveau } 3 \text { de la CITE) } \\
\text { (§168). }\end{array}$ & $\begin{array}{l}\text { Enseignants souvent plus qualifiés dans la } \\
\text { matière qu'ils enseignent que les enseignants } \\
\text { du premier cycle du secondaire }(§ 170) \text {. }\end{array}$ \\
\hline & & $\begin{array}{l}\text { Durée cumulée : programmes se terminent } \\
12 \text { ou } 13 \text { ans après le début de la CITE } 1 \\
\text { (§164). }\end{array}$ & \\
\hline \multirow{3}{*}{4} & \multirow{3}{*}{$\begin{array}{l}\text { Enseignement } \\
\text { post-secondaire } \\
\text { non-supérieur }\end{array}$} & $\begin{array}{l}\text { Enseignement post-secondaire, généralement } \\
\text { des programmes professionnels et terminaux } \\
\text { préparant au marché du travail ; le plus souvent, } \\
\text { pas considéré comme de l'enseignement } \\
\text { supérieur au niveau national ( } \$ 190) \text {. }\end{array}$ & \\
\hline & & $\begin{array}{l}\text { Programmes qui servent à élargir plutôt qu'à } \\
\text { approfondir les connaissances, aptitudes et } \\
\text { compétences des participants. Souvent pas } \\
\text { d'un niveau significativement plus avancé que } \\
\text { les programmes du niveau } 3 \text { de la CITE (§191). }\end{array}$ & \\
\hline & & $\begin{array}{l}\text { Critères d'admission : achèvement du deuxième } \\
\text { cycle de l'enseignement secondaire ( } \$ 186) \text {. }\end{array}$ & \\
\hline \multirow{3}{*}{5} & \multirow{3}{*}{$\begin{array}{l}\text { Enseignement } \\
\text { supérieur de cycle } \\
\text { court }\end{array}$} & $\begin{array}{l}\text { Programmes souvent destinés à enseigner } \\
\text { aux participants des connaissances, } \\
\text { aptitudes et compétences professionnelles ; } \\
\text { peuvent représenter une passerelle vers } \\
\text { des programmes académiques ( } \$ 207) \text {. Plus } \\
\text { complexes que les niveaux } 3 \text { et } 4 \text { mais moins } \\
\text { que le } 6(\S 212) \text {. }\end{array}$ & \multirow[t]{2}{*}{$\begin{array}{l}\text { Points de transition institutionnels : souvent } \\
\text { dispensés dans des établissements } \\
\text { d'enseignement différents de ceux qui offrent } \\
\text { des programmes de niveaux } 6,7 \text { et } 8 \text { ( } \$ 214) \text {. }\end{array}$} \\
\hline & & $\begin{array}{l}\text { Critères d'admission : achèvement complet } \\
\text { du deuxième cycle du secondaire ou de } \\
\text { l'enseignement post-secondaire non- } \\
\text { supérieur donnant accès aux niveaux } 5,6 \text { ou } \\
7 \text { de la CITE (\$208). }\end{array}$ & \\
\hline & & Durée minimale : 2 ans (\$213). & Durée typique : 2 à 3 ans (§213). \\
\hline
\end{tabular}

\section{Notes}

* Les numéros de paragraphe constituent des références à la classification CITE 2011. Pour plus de détails, veuillez consulter le Guide du lecteur.

** Variable HATLEVEL / HATVOC de l'enquête de l'Union européenne sur la population active (Règlement de la Commission européenne 317/2013). 


\begin{tabular}{|c|c|c|c|c|c|c|}
\hline \multicolumn{4}{|c|}{ Dimensions complémentaires } & \multicolumn{3}{|l|}{ Codification } \\
\hline \multicolumn{2}{|c|}{$2^{e}$ chiffre } & \multicolumn{2}{|c|}{$3^{e}$ chiffre } & $\begin{array}{l}\text { Programmes } \\
\text { éducatifs } \\
\text { CITE-P (Annexe } \\
\text { II de la CITE } \\
\text { 2011) }\end{array}$ & $\begin{array}{l}\text { Niveau } \\
\text { d'éducation } \\
\text { atteint CITE-A } \\
\text { (Annexe III de } \\
\text { la CITE 2011) }\end{array}$ & $\begin{array}{l}\text { Variable } \\
\text { HATLEVEL / } \\
\text { HATVOC** } \\
\text { de l'enquête } \\
\text { de l'Union } \\
\text { européenne sur } \\
\text { la population } \\
\text { active }\end{array}$ \\
\hline \multicolumn{2}{|c|}{$\begin{array}{l}\text { Orientation du } \\
\text { programme : }\end{array}$} & \multicolumn{2}{|c|}{$\begin{array}{l}\text { Achèvement de niveau et accès au niveau } \\
\text { supérieur de la CITE : }\end{array}$} & & & \\
\hline \multirow[t]{2}{*}{4} & \multirow[t]{2}{*}{ Général } & 1 & $\begin{array}{l}\text { Insuffisant pour un achèvement du } \\
\text { niveau ou achèvement partiel d'un } \\
\text { niveau (durée }<2 \text { ans ou durée } \\
\text { cumulée }<11 \text { ans depuis le début du } \\
\text { niveau } 1 \text { de la CITE). }\end{array}$ & 341,351 & 244,254 & 200 \\
\hline & & 2 & $\begin{array}{l}\text { Achèvement partiel d'un niveau } \\
\text { (programme intermédiaire avec durée } \\
\geq 2 \text { ans et durée cumulée } \geq 11 \text { ans). }\end{array}$ & 342,352 & 342,352 & $302 / 1,302 / 2$ \\
\hline \multirow{2}{*}{5} & \multirow{2}{*}{ Professionnel } & 3 & $\begin{array}{l}\text { Achèvement d'un niveau sans accès } \\
\text { direct à la CITE } 3 \text { (durée } \geq 2 \text { ans, durée } \\
\text { cumulée } \geq 11 \text { ans). }\end{array}$ & 343,353 & 343,353 & $303 / 1,303 / 2$ \\
\hline & & 4 & $\begin{array}{l}\text { Achèvement d'un niveau avec accès } \\
\text { direct à la CITE } 5,6 \text { ou } 7 \text { (durée } \\
\geq 2 \text { ans, durée cumulée } \geq 11 \text { ans). }\end{array}$ & 344,354 & 344,354 & $304 / 1,304 / 2$ \\
\hline \multicolumn{2}{|c|}{$\begin{array}{l}\text { Orientation du } \\
\text { programme : }\end{array}$} & \multicolumn{2}{|c|}{$\begin{array}{l}\text { Achèvement de niveau et accès au niveau } \\
\text { supérieur de la CITE : }\end{array}$} & & & \\
\hline 4 & Général & 1 & $\begin{array}{l}\text { Insuffisant pour un achèvement du } \\
\text { niveau (durée }<6 \text { mois) }\end{array}$ & 441,451 & 344354 & $300 / 1,300 / 2$ \\
\hline \multirow{2}{*}{5} & \multirow{2}{*}{ Professionnel } & 3 & $\begin{array}{l}\text { Achèvement d'un niveau sans accès } \\
\text { direct à la CITE } 5,6 \text { ou } 7\end{array}$ & 443,453 & 443,453 & $400 / 1,400 / 2$ \\
\hline & & 4 & $\begin{array}{l}\text { Achèvement de niveau avec accès } \\
\text { direct à la CITE } 5,6 \text { ou } 7\end{array}$ & 444,454 & 444,454 & \\
\hline \multicolumn{2}{|c|}{$\begin{array}{l}\text { Orientation du } \\
\text { programme : }\end{array}$} & \multicolumn{2}{|c|}{$\begin{array}{l}\text { Achèvement de niveau et accès au niveau } \\
\text { supérieur de la CITE : }\end{array}$} & & & \\
\hline 4 & $\begin{array}{l}\text { Général } \\
\text { (ou académique) }\end{array}$ & 1 & $\begin{array}{l}\text { Insuffisant pour un achèvement du } \\
\text { niveau (durée }<2 \text { ans) }\end{array}$ & 541,551 & 444,454 & 400 \\
\hline 5 & Professionnel & 4 & Achèvement de niveau & 544,554 & 540,550 & 500 \\
\hline
\end{tabular}

\section{Notes}

* Les numéros de paragraphe constituent des références à la classification CITE 2011. Pour plus de détails, veuillez consulter le Guide du lecteur.

** Variable HATLEVEL / HATVOC de l'enquête de l'Union européenne sur la population active (Règlement de la Commission européenne 317/2013). 


\begin{tabular}{|c|c|c|c|}
\hline \multirow{2}{*}{\multicolumn{2}{|c|}{$\begin{array}{l}\text { Niveau } \\
1^{\text {er }} \text { chiffre }\end{array}$}} & \multicolumn{2}{|c|}{ Critères de classification des programmes nationaux par niveaux } \\
\hline & & Critères principaux & Critères subsidiaires \\
\hline \multirow{4}{*}{6} & \multirow{4}{*}{$\begin{array}{l}\text { Licence } \\
\text { ou équivalent }\end{array}$} & $\begin{array}{l}\text { Programmes souvent destinés à enseigner } \\
\text { aux participants des connaissances, } \\
\text { aptitudes et compétences académiques et/ou } \\
\text { professionnelles intermédiaires conduisant à } \\
\text { un premier diplôme tel qu'une Licence ou une } \\
\text { certification équivalente (\$224). }\end{array}$ & $\begin{array}{l}\text { L'exigence d'un doctorat (niveau } 8 \text { de la } \\
\text { CITE) pour certains membres du personnel } \\
\text { enseignant peut contribuer à la distinction } \\
\text { entre les niveaux } 5 \text { et } 6 \text { de la CITE (\$231). }\end{array}$ \\
\hline & & $\begin{array}{l}\text { Critères d'admission : achèvement complet } \\
\text { du deuxième cycle du secondaire ou de } \\
\text { l'enseignement post-secondaire non- } \\
\text { supérieur donnant accès aux niveaux } 5,6 \text { ou } \\
7 \text { de la CITE ; peut nécessiter la réussite d'un } \\
\text { examen d'entrée ( } \$ 226) \text {. }\end{array}$ & \multirow[t]{3}{*}{$\begin{array}{l}\text { Études ultérieures : ne donne pas un accès } \\
\text { direct (généralement) à des programmes de } \\
\text { niveau doctorat (niveau } 8 \text { de la CITE) (\$226) }\end{array}$} \\
\hline & & $\begin{array}{l}\text { Durée cumulée minimale des premiers } \\
\text { diplômes : } 3 \text { à } 4 \text { ans à temps plein (\$229). }\end{array}$ & \\
\hline & & $\begin{array}{l}\text { Position dans la structure nationale des } \\
\text { diplômes : généralement un premier diplôme } \\
\text { dans l'enseignement supérieur ; parfois un } \\
\text { deuxième diplôme de } 1 \text { à } 2 \text { ans ( }(230) \text {. }\end{array}$ & \\
\hline \multirow{3}{*}{7} & \multirow{3}{*}{$\begin{array}{l}\text { Master } \\
\text { ou équivalent }\end{array}$} & $\begin{array}{l}\text { Programmes souvent destinés à enseigner } \\
\text { aux participants des connaissances, } \\
\text { aptitudes et compétences académiques et/ } \\
\text { ou professionnelles avancées conduisant à } \\
\text { un deuxième diplôme tel qu'un master ou une } \\
\text { certification équivalente (\$241). }\end{array}$ & $\begin{array}{l}\text { Durée minimale du premier diplôme long : } \\
5 \text { ans; complexité du contenu comparable à } \\
\text { celle d'un master (\$247). }\end{array}$ \\
\hline & & $\begin{array}{l}\text { Position dans la structure nationale des } \\
\text { diplômes : généralement un deuxième diplôme } \\
\text { ou diplôme supplémentaire dans l'enseignement } \\
\text { supérieur succédant à un premier diplôme au } \\
\text { niveau } 6 \text { ou } 7 \text { de la CITE (\$246) ou un premier } \\
\text { diplôme long d'au moins } 5 \text { ans si équivalent à } \\
\text { un master en termes de complexité du contenu } \\
\text { (exemple médecine) (\$247). }\end{array}$ & \multirow[t]{2}{*}{$\begin{array}{l}\text { Études ultérieures : donne souvent un accès } \\
\text { direct à des programmes de niveau doctorat } \\
\text { (niveau } 8 \text { de la CITE) (\$249). }\end{array}$} \\
\hline & & $\begin{array}{l}\text { Critères d'admission : dans le cas d'un } 2^{\mathrm{e}} \\
\text { diplôme, l'achèvement complet d'une licence ou } \\
\text { équivalent (niveau } 6 \text { de la CITE) ou d'un master } \\
\text { ou équivalent (niveau } 7 \text { de la CITE) est exigé ; } \\
\text { dans le cas d'un 1er diplôme, l'achèvement } \\
\text { complet du deuxième cycle du secondaire ou } \\
\text { de la CITE } 4 \text { donnant accès à l'enseignement } \\
\text { supérieur est exigé et éventuellement la réussite } \\
\text { d'un examen d'entrée (\$243). }\end{array}$ & \\
\hline \multirow{3}{*}{8} & \multirow{3}{*}{$\begin{array}{l}\text { Doctorat } \\
\text { ou équivalent }\end{array}$} & $\begin{array}{l}\text { Son achèvement complet exige la remise } \\
\text { d'une thèse ou d'un travail écrit équivalent } \\
\text { d'une qualité suffisante pour en permettre la } \\
\text { publication, qui représente le produit de travaux } \\
\text { de recherche originaux de même qu'une } \\
\text { contribution appréciable aux connaissances } \\
\text { dans le domaine d'études (\$264). }\end{array}$ & \multirow[t]{2}{*}{$\begin{array}{l}\text { Diplôme donnant accès à des postes } \\
\text { d'enseignants et de chercheurs ( } \$ 266) \text {. }\end{array}$} \\
\hline & & $\begin{array}{l}\text { Critères d'admission : l'achèvement complet } \\
\text { d'un programme CITE } 7 \text { (§261). }\end{array}$ & \\
\hline & & $\begin{array}{l}\text { Durée minimale : au moins } 3 \text { ans d'études à } \\
\text { temps plein et une durée cumulée totale d'au } \\
\text { moins } 7 \text { ans dans l'enseignement supérieur } \\
\text { (§265) }\end{array}$ & \\
\hline
\end{tabular}

\section{Notes}

* Les numéros de paragraphe constituent des références à la classification CITE 2011. Pour plus de détails, veuillez consulter le Guide du lecteur.

** Variable HATLEVEL / HATVOC de l'enquête de l'Union européenne sur la population active (Règlement de la Commission européenne 317/2013) 


\begin{tabular}{|c|c|c|c|c|c|c|}
\hline \multicolumn{4}{|c|}{ Dimensions complémentaires } & \multicolumn{3}{|l|}{ Codification } \\
\hline \multicolumn{2}{|c|}{$2^{\text {ème }}$ chiffre } & \multicolumn{2}{|c|}{$3^{\text {ème }}$ chiffre } & $\begin{array}{l}\text { Programmes } \\
\text { éducatifs } \\
\text { CITE-P } \\
\text { (Annexe II } \\
\text { de la CITE } \\
\text { 2011) }\end{array}$ & $\begin{array}{l}\text { Niveau } \\
\text { d'éducation } \\
\text { atteint } \\
\text { CITE-A } \\
\text { (Annexe III de } \\
\text { la CITE 2011) }\end{array}$ & $\begin{array}{l}\text { Variable HATLEVEL } \\
\text { / HATVOC** } \\
\text { de l'enquête } \\
\text { de l'Union } \\
\text { européenne sur la } \\
\text { population active }\end{array}$ \\
\hline \multicolumn{2}{|c|}{$\begin{array}{l}\text { Orientation du } \\
\text { programme : }\end{array}$} & \multicolumn{2}{|c|}{$\begin{array}{l}\text { Position dans la structure nationale } \\
\text { des diplômes et certifications : }\end{array}$} & & & \\
\hline 4 & Académique & 1 & $\begin{array}{l}\text { Insuffisant comme achèvement } \\
\text { de niveau (durée du premier } \\
\text { diplôme }<3 \text { ans) }\end{array}$ & $\begin{array}{c}641,651 \\
661\end{array}$ & 540,550 & 500 \\
\hline 5 & Professionnel & 5 & $\begin{array}{l}\text { Premier diplôme (au niveau de la } \\
\text { licence) (durée de 3-4 ans) }\end{array}$ & $\begin{array}{l}645,655 \\
665\end{array}$ & \multirow{3}{*}{$640,650,660$} & \multirow{3}{*}{600} \\
\hline \multirow{2}{*}{6} & \multirow{2}{*}{ Non spécifié } & 6 & $\begin{array}{l}\text { Premier degré long (au niveau de } \\
\text { la licence) (durée> } 4 \text { ans) }\end{array}$ & $\begin{array}{c}646,656 \\
666\end{array}$ & & \\
\hline & & & $\begin{array}{l}\text { Deuxième diplôme ou diplôme } \\
\text { supplémentaire (après un } 1^{\mathrm{er}} \\
\text { diplôme au niveau licence) }\end{array}$ & $\begin{array}{c}647,657 \\
667\end{array}$ & & \\
\hline \multicolumn{2}{|c|}{$\begin{array}{l}\text { Orientation du } \\
\text { programme : }\end{array}$} & \multicolumn{2}{|c|}{$\begin{array}{l}\text { Position dans la structure nationale } \\
\text { des diplômes et certifications : }\end{array}$} & & & \\
\hline 4 & Académique & 1 & $\begin{array}{l}\text { Insuffisant comme achèvement } \\
\text { de niveau (durée du premier } \\
\text { diplôme }<5 \text { ans) }\end{array}$ & $\begin{array}{l}741,751 \\
761\end{array}$ & $640,650,660$ & 600 \\
\hline 5 & Professionnel & 6 & $\begin{array}{l}\text { Premier diplôme long (au niveau } \\
\text { Master (durée>= } 5 \text { ans) }\end{array}$ & $\begin{array}{l}746,756 \\
766\end{array}$ & \multirow{3}{*}{$740,750,760$} & \multirow{3}{*}{700} \\
\hline \multirow{2}{*}{6} & \multirow{2}{*}{ Non spécifié } & 7 & $\begin{array}{l}\text { Deuxième diplôme ou diplôme } \\
\text { supplémentaire (après un 1er } \\
\text { diplôme au niveau licence) }\end{array}$ & $\begin{array}{c}747,757 \\
767\end{array}$ & & \\
\hline & & 8 & $\begin{array}{l}\text { Deuxième diplôme ou diplôme } \\
\text { supplémentaire (après un } 1^{\mathrm{er}} \\
\text { diplôme au niveau Master) }\end{array}$ & $\begin{array}{l}748,758 \\
768\end{array}$ & & \\
\hline \multicolumn{2}{|c|}{$\begin{array}{l}\text { Orientation du } \\
\text { programme : }\end{array}$} & \multicolumn{2}{|c|}{$\begin{array}{l}\text { Position dans la structure nationale } \\
\text { des diplômes et certifications : }\end{array}$} & & & \\
\hline 4 & Académique & \multirow[t]{2}{*}{1} & \multirow{2}{*}{$\begin{array}{l}\text { Insuffisant comme achèvement } \\
\text { de niveau (durée du premier } \\
\text { diplôme }<3 \text { ans) }\end{array}$} & $\begin{array}{c}841,851 \\
861\end{array}$ & $740,750,760$ & 700 \\
\hline 5 & Professionnel & & & \multirow{2}{*}{$\begin{array}{c}844,854 \\
864\end{array}$} & \multirow{2}{*}{$840,850,860$} & \multirow{2}{*}{800} \\
\hline 6 & Non spécifié & 4 & Achèvement de niveau & & & \\
\hline
\end{tabular}

\section{Notes}

* Les numéros de paragraphe constituent des références au document principal classification CITE 2011. Pour plus de plus de détails, veuillez consulter le Guide du lecteur.

** Variable HATLEVEL / HATVOC de l'Enquête sur la population active de l'Union européenne (règlement 317/2013 de la Commission européenne). 



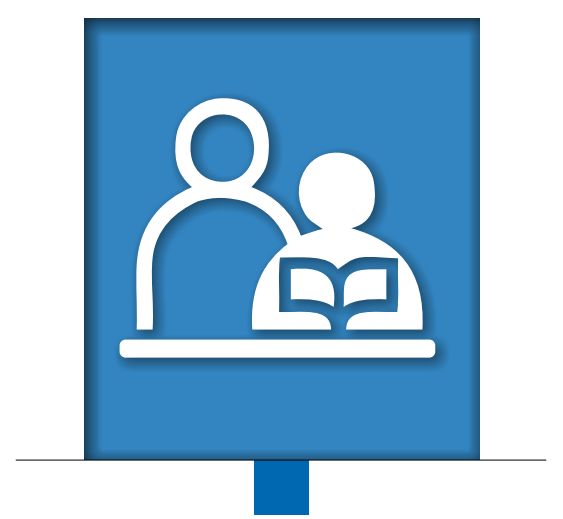

Annexe B

\section{Parcours scolaires potentiels de la CITE 2011}


PARCOURS POTENTIELS DE LA CITE 2011 AVEC TOUS LES CODES CITE-P

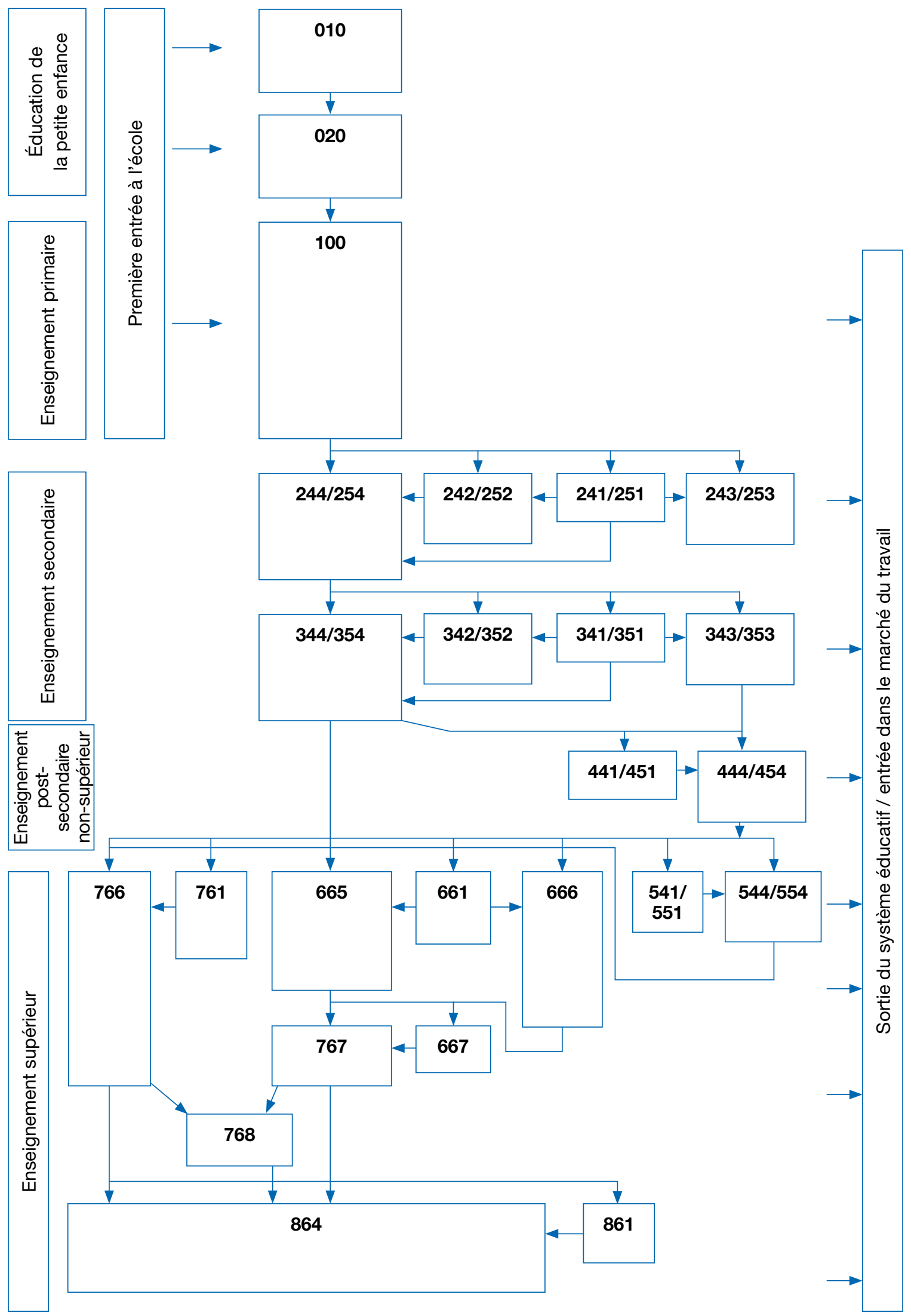




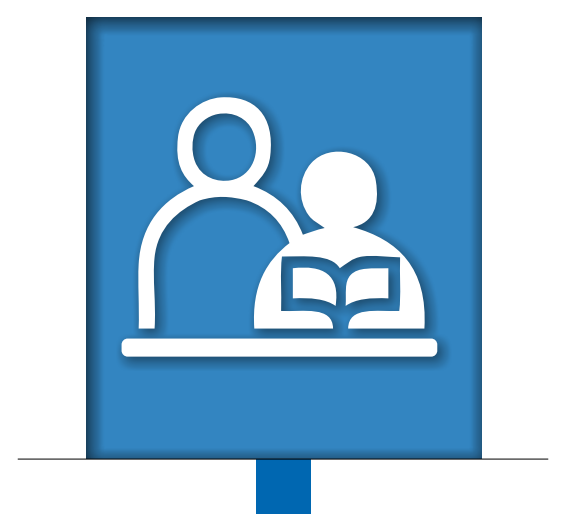

Annexe C

\section{Référence rapide des codes de la CITE-P et de la CITE-A dans la CITE 2011}




\begin{tabular}{|c|c|c|c|}
\hline CITE-P & \multicolumn{2}{|l|}{ Notes sur la codification } & CITE-A \\
\hline (sans objet) & (sans objet) & N'a jamais participé à un programme éducatif & 010 \\
\hline 010 & Développement éducatif de la petite enfance & Éducation de la petite enfance limitée & 020 \\
\hline \multirow[t]{2}{*}{020} & Enseignement préprimaire & Éducation de la petite enfance limitée & 020 \\
\hline & & $\begin{array}{l}\text { Scolarisation primaire limitée (sans } \\
\text { achèvement de niveau) }\end{array}$ & 030 \\
\hline 100 & \multicolumn{2}{|l|}{ Enseignement primaire } & 100 \\
\hline $241 / 251$ & \multicolumn{2}{|c|}{$\begin{array}{l}\text { Premier cycle du secondaire, insuffisant pour un achèvement complet ou partiel de } \\
\text { niveau, sans accès direct au deuxième cycle de l'enseignement secondaire }\end{array}$} & 100 \\
\hline $242 / 252$ & \multicolumn{2}{|c|}{$\begin{array}{l}\text { Premier cycle du secondaire, achèvement partiel de niveau, sans accès direct au } \\
\text { deuxième cycle de l'enseignement secondaire }\end{array}$} & $242 / 252$ \\
\hline $243 / 253$ & \multicolumn{2}{|c|}{$\begin{array}{l}\text { Premier cycle du secondaire, achèvement de niveau, sans accès direct au deuxième cycle } \\
\text { de l'enseignement secondaire }\end{array}$} & $243 / 253$ \\
\hline $244 / 254$ & \multicolumn{2}{|c|}{$\begin{array}{l}\text { Premier cycle du secondaire, achèvement de niveau, avec accès direct au deuxième cycle } \\
\text { de l'enseignement secondaire }\end{array}$} & $244 / 254$ \\
\hline $341 / 351$ & \multicolumn{2}{|c|}{$\begin{array}{l}\text { Deuxième cycle du secondaire, insuffisant pour un achèvement complet ou partiel de } \\
\text { niveau, sans accès direct à l'enseignement supérieur }\end{array}$} & $244 / 254$ \\
\hline $342 / 352$ & \multicolumn{2}{|c|}{$\begin{array}{l}\text { Deuxième cycle du secondaire, achèvement partiel de niveau, sans accès direct à } \\
\text { l'enseignement supérieur }\end{array}$} & $342 / 352$ \\
\hline $343 / 353$ & \multicolumn{2}{|c|}{$\begin{array}{l}\text { Deuxième cycle du secondaire, achèvement de niveau, sans accès direct à } \\
\text { l'enseignement supérieur }\end{array}$} & $343 / 353^{*}$ \\
\hline $344 / 354$ & \multicolumn{2}{|c|}{$\begin{array}{l}\text { Deuxième cycle du secondaire, achèvement de niveau, avec accès direct à } \\
\text { l'enseignement supérieur }\end{array}$} & $344 / 354$ \\
\hline $441 / 451$ & \multicolumn{2}{|c|}{$\begin{array}{l}\text { Post-secondaire non-supérieur, insuffisant pour un achèvement de niveau, sans accès } \\
\text { direct à l'enseignement supérieur }\end{array}$} & $344 / 354$ \\
\hline $443 / 453$ & \multicolumn{2}{|c|}{$\begin{array}{l}\text { Post-secondaire non-supérieur, achèvement de niveau, sans accès direct à } \\
\text { l'enseignement supérieur }\end{array}$} & $443 / 453^{*}$ \\
\hline $444 / 454$ & \multicolumn{2}{|c|}{$\begin{array}{l}\text { Post-secondaire non-supérieur, achèvement de niveau, avec accès direct à } \\
\text { l'enseignement supérieur }\end{array}$} & $444 / 454$ \\
\hline $541 / 551$ & \multicolumn{2}{|c|}{ Enseignement supérieur de cycle court, insuffisant comme achèvement de niveau } & $444 / 454$ \\
\hline $544 / 554$ & \multicolumn{2}{|c|}{ Enseignement supérieur de cycle court, achèvement de niveau } & $540 / 550$ \\
\hline $641 / 651 / 661$ & \multicolumn{2}{|c|}{ Niveau licence ou équivalent, insuffisant pour un achèvement de niveau } & $540 / 550 / 560$ \\
\hline $645 / 655 / 665$ & \multicolumn{2}{|c|}{ Niveau licence ou équivalent, premier diplôme (3 à 4 ans) } & $640 / 650 / 660$ \\
\hline $646 / 656 / 666$ & \multicolumn{2}{|c|}{ Niveau licence ou équivalent, premier diplôme long (plus de 4 ans) } & $640 / 650 / 660$ \\
\hline $647 / 657 / 667$ & \multicolumn{2}{|c|}{$\begin{array}{l}\text { Niveau licence ou équivalent, deuxième diplôme ou diplôme supplémentaire après un } \\
\text { programme de licence ou équivalent }\end{array}$} & $640 / 650 / 660$ \\
\hline $741 / 751 / 761$ & \multicolumn{2}{|c|}{ Niveau master ou équivalent, insuffisant pour un achèvement de niveau } & $640 / 650 / 660$ \\
\hline $746 / 756 / 766$ & \multicolumn{2}{|c|}{ Niveau master ou équivalant, premier diplôme long (au moins 5 ans) } & $740 / 750 / 760$ \\
\hline $747 / 757 / 767$ & \multicolumn{2}{|c|}{$\begin{array}{l}\text { Niveau master ou équivalent, deuxième diplôme ou diplôme supplémentaire après une } \\
\text { licence ou équivalent }\end{array}$} & $740 / 750 / 760$ \\
\hline $748 / 758 / 768$ & \multicolumn{2}{|c|}{$\begin{array}{l}\text { Niveau master ou équivalent, deuxième diplôme ou diplôme supplémentaire après un } \\
\text { master ou équivalent }\end{array}$} & $740 / 750 / 760$ \\
\hline $841 / 851 / 861$ & \multicolumn{2}{|c|}{ Niveau doctorat ou équivalent, insuffisant pour un achèvement de niveau } & $740 / 750 / 760$ \\
\hline $844 / 854 / 864$ & \multicolumn{2}{|c|}{ Niveau doctorat ou équivalent, achèvement de niveau } & $840 / 850 / 860$ \\
\hline
\end{tabular}

\section{Note}

* La CITE-A des programmes 343/353 et 443/453 (CITE-P) peut aussi être 344/354 et 444/454 respectivement si le critère minimal d'admission au programme incluait déjà un accès à l'enseignement supérieur (même si le programme en lui-même est destiné à mener au marché du travail et non à l'enseignement supérieur). 


\section{UNESCO}

L'Acte constitutif de l'Organisation des Nations Unies pour l'éducation, la science et la culture (UNESCO) a été adopté par 20 pays lors de la Conférence de Londres en novembre 1945 et est entré en vigueur le 4 novembre 1946. L'UNESCO compte aujourd'hui 195 États membres et 9 membres associés.

L'objectif premier de l'UNESCO est de contribuer au maintien de la paix et de la sécurité dans le monde en resserrant, par l'éducation, la science et la culture, la collaboration entre nations afin d'assurer le respect universel de la justice, de la loi, des droits de l'homme et des libertés fondamentales pour tous, sans distinction de race, de sexe, de langue ou de religion, que la Charte des Nations Unies reconnaît à tous les peuples.

L'UNESCO a cinq fonctions principales, inscrites dans son mandat : 1) des études prospectives sur l'éducation, la science, la culture et la communication dans le monde de demain ; 2) le progrès, le transfert et le partage des connaissances par des activités de recherche, de formation et d'enseignement ; 3) des actions normatives en vue de la préparation et de l'adoption d'instruments internationaux et de recommandations réglementaires ; 4) l'expertise par le biais de la coopération technique avec les États membres, en faveur de leurs projets et politiques de développement ; et 5) l'échange d'informations spécialisées.

Le siège de I'UNESCO se situe à Paris, en France.

\section{INSTITUT DE STATISTIQUE DE L'UNESCO}

L'Institut de statistique de I'UNESCO (ISU) est l'office de statistique de I'UNESCO. II est chargé de rassembler, pour le compte des Nations Unies, des statistiques mondiales dans les domaines de l'éducation, de la science et la technologie, de la culture et la communication.

L'ISU a été créé en 1999 avec pour mission d'améliorer le programme statistique de I'UNESCO et d'élaborer et mettre à disposition des statistiques actualisées, précises et pertinentes pour les politiques, comme l'exige le contexte social, politique et économique actuel de plus en plus complexe et changeant.

Le siège de I'ISU se situe à Montréal, au Canada.

\section{ORGANISATION DE COOPÉRATION ET DÉVELOPPEMENT ÉCONOMIQUES}

L'OCDE est un forum unique en son genre où les gouvernements œuvrent ensemble pour relever les défis économiques, sociaux et environnementaux liés à la mondialisation. À l'avant-garde des efforts engagés pour comprendre les évolutions du monde actuel et les préoccupations qu'elles suscitent, l'OCDE aide les gouvernements à y faire face en menant une réflexion sur des thèmes tels que le gouvernement d'entreprise, l'économie de l'information et la problématique du vieillissement démographique. L'Organisation offre aux gouvernements un cadre leur permettant de confronter leurs expériences en matière d'action publique, de chercher des réponses à des problèmes communs, de recenser les bonnes pratiques et de travailler à la coordination des politiques nationales et internationales.

Les pays membres de l'OCDE sont : I'Allemagne, l'Australie, l'Autriche, la Belgique, le Canada, le Chili, la Corée, le Danemark, l'Espagne, l'Estonie, les États-Unis, la Finlande, la France, la Grèce, la Hongrie, I'Irlande, I'Islande, Israël, I'Italie, le Japon, le Luxembourg, le Mexique, la Norvège, la Nouvelle-Zélande, les Pays-Bas, la Pologne, le Portugal, la République slovaque, la République tchèque, le Royaume-Uni, la Slovénie, la Suède, la Suisse et la Turquie. L'Union européenne participe aux travaux de l'OCDE.

Les Éditions OCDE assurent une large diffusion aux travaux de l'Organisation. Ces derniers comprennent les résultats de l'activité de collecte de statistiques, les travaux de recherche menés sur des questions économiques, sociales et environnementales, ainsi que les conventions, les principes directeurs et les modèles développés par les pays membres.

\section{EUROSTAT}

Eurostat est le bureau de statistiques de l'Union européenne (UE). Il est la source reconnue des statistiques européennes officielles et permet des comparaisons entre les 28 États membres de l'UE, les pays membres de l'Association européenne de libre-échange (AELE), les pays candidats à l'adhésion à l'UE et d'autres pays voisins de l'UE, ainsi qu'entre les régions de ces pays. Eurostat coopère étroitement avec les autorités nationales responsables des statistiques dans l'UE et les pays AELE par l'intermédiaire du Système statistique européen (SSE). Le SSE, développé progressivement en vue de fournir des statistiques comparables au niveau de l'UE, fonctionne comme un réseau dans lequel le rôle d'Eurostat est de diriger l'harmonisation des statistiques. II travaille en coordination avec les pays candidats à l'adhésion à l'UE, les agences et la BCE, ainsi qu'avec des organisations internationales telles que l'OCDE, les NU, le Fonds monétaire international et la Banque mondiale. Les données et les publications d'Eurostat sont accessibles gratuitement à http://ec.europa.eu/eurostat. 




\section{Guide opérationnel CITE 2011 :}

\section{DIRECTIVES POUR LA CLASSIFICATION DES PROGRAMMES ÉDUCATIFS NATIONAUX ET DES CERTIFICATIONS CORRESPONDANTES}

La structure des systèmes éducatifs varie énormément d'un pays à l'autre. Afin de produire des statistiques et des indicateurs comparables à l'échelle internationale, il est nécessaire de disposer d'un cadre permettant de collecter et de soumettre des données sur des programmes éducatifs avec un contenu éducatif de niveau similaire. La Classification internationale type de l'Éducation (CITE) de l'UNESCO est la classification de référence permettant d'organiser les programmes éducatifs et les certifications correspondantes par niveau d'éducation et par domaines d'études. Les définitions et les concepts fondamentaux de la CITE ont été établis de manière à être internationalement valides et applicables à l'ensemble des systèmes éducatifs.

La CITE 2011 est la deuxième révision importante de cette classification (élaborée initialement dans les années 70 et révisée pour la première fois en 1997). Elle a été adoptée par la Conférence générale de l'UNESCO en novembre 2011. Préparé conjointement par l'Institut de statistique de I'UNESCO (ISU), I'OCDE et Eurostat, ce guide opérationnel fournit des directives et notes explicatives pour l'interprétation de la classification révisée, par niveau éducatif. II présente également des exemples nationaux de programmes et de certifications correspondantes classés dans la CITE 2011.

Ce guide sera utile aux statisticiens nationaux qui collectent et soumettent des données d'éducation aux organisations internationales, ainsi qu'aux décideurs politiques et aux chercheurs intéressés par une meilleure compréhension de ces données.

\section{Table des matières}

Chapitre 1. Aperçu de la CITE 2011

Chapitre 2. Niveau 0 de la CITE 2011 : Éducation de la petite enfance

Chapitre 3. Niveau 1 de la CITE 2011 : Enseignement primaire

Chapitre 4. Niveau 2 de la CITE 2011 : Premier cycle de l'enseignement secondaire

Chapitre 5. Niveau 3 de la CITE 2011 : Deuxième cycle de l'enseignement secondaire

Chapitre 6. Niveau 4 de la CITE 2011 : Enseignement post-secondaire non-supérieur

Chapitre 7. Aperçu des niveaux de l'enseignement supérieur de la CITE 2011

Chapitre 8. Niveau 5 de la CITE 2011 : Enseignement supérieur de cycle court

Chapitre 9. Niveau 6 de la CITE 2011 : Niveau licence ou équivalent

Chapitre 10. Niveau 7 de la CITE 2011 : Niveau master ou équivalent

Chapitre 11. Niveau 8 de la CITE 2011 : Niveau doctorat ou équivalent 